Florida International University

FIU Digital Commons

3-17-2020

\title{
Artificial Intelligent Based Energy and Demand Side Management for Microgrids and Smart Homes Considering Customer Privacy
}

AHMED F. EBRAHIM

aebra003@fiu.edu

Follow this and additional works at: https://digitalcommons.fiu.edu/etd

Part of the Electrical and Electronics Commons, Other Electrical and Computer Engineering Commons, and the Power and Energy Commons

\section{Recommended Citation}

EBRAHIM, AHMED F., "Artificial Intelligent Based Energy and Demand Side Management for Microgrids and Smart Homes Considering Customer Privacy" (2020). FIU Electronic Theses and Dissertations. 4424. https://digitalcommons.fiu.edu/etd/4424

This work is brought to you for free and open access by the University Graduate School at FIU Digital Commons. It has been accepted for inclusion in FIU Electronic Theses and Dissertations by an authorized administrator of FIU Digital Commons. For more information, please contact dcc@fiu.edu. 


\section{FLORIDA INTERNATIONAL UNIVERSITY}

Miami, Florida

\section{ARTIFICIAL INTELLIGENCE BASED ENERGY AND DEMAND SIDE MANAGEMENT FOR MICROGRIDS AND SMART HOMES CONSIDERING CUSTOMER PRIVACY}

A dissertation submitted in partial fulfillment of the requirements for the degree of DOCTOR OF PHILOSOPHY in ELECTRICAL AND COMPUTER ENGINEERING

by

Ahmed Faragallah Farid Ebrahim 
To: Dean John L. Volakis

College of Engineering and Computing

This dissertation, written by Ahmed Faragallah Farid Ebrahim, and entitled Artificial Intelligence Based Energy and Demand Side Management for Microgrids and Smart Homes Considering Customer Privacy, having been approved in respect to style and intellectual content, is referred to you for judgment.

We have read this dissertation and recommend that it be approved.

$\begin{array}{r}\text { Ahmed Ibrahim } \\ \hline \text { Mohammed Hadi } \\ \hline \text { Sakhrat Khizroev } \\ \hline \text { Osama A. Mohammed, Major Professor }\end{array}$

Date of Defense: March 17, 2020

The dissertation of Ahmed Faragallah Farid Ebrahim is approved.

Dean John L. Volakis

College of Engineering and Computing

Andrés G. Gil

Vice President for Research and Economic Development and Dean of the University Graduate School

Florida International University, 2020 
(C) Copyright 2020 by Ahmed Faragallah Farid Ebrahim.

All rights reserved. 


\section{DEDICATION}

For their endless support, love, and sacrifice, I dedicate this work.

To my Beloved Parents, my Brothers, my kids and Dear Wife Amira. 


\section{ACKNOWLEDGMENTS}

I would like to thank my research supervisor, Professor Osama Mohammed, for supervising me in this work, and for providing me with endless research ideas and technical support. He also helped providing me with financial support through a teaching assistantship in the ECE Department and working in his research group. I would like to thank him for making me a part of the Energy System Research Laboratory (ESRL) and for the excellent facilities made available for this projects in this dissertation. I have gained a lot of skills and experience at ESRL with its first-class equipment needed to build and experimentally verify the results. This helped me complete my doctoral studies and enabled scholarly production resulting from this project. Also, as my doctoral research started to gain traction, Professor Mohammed provided me opportunities to grow within my professional career inside and outside the university.

I would also like to thank my committee members for their support. I also like to acknowledge the support for the FIU graduate school for providing me with the dissertation year fellowship during my final year in the program.

Finally, thanks to all my colleagues at the Energy Systems Research Laboratory, who created a professional and collaborative environment that helped me reach my research goals. 


\author{
ABSTRACT OF THE DISSERTATION \\ ARTIFICIAL INTELLIGENCE BASED ENERGY AND DEMAND SIDE \\ MANAGEMENT FOR MICROGRIDS AND SMART HOMES CONSIDERING \\ CUSTOMER PRIVACY \\ by
}

Ahmed F. Ebrahim

Florida International University, 2020

Miami, Florida, USA

Professor Osama A. Mohammed, Major Professor

The rapid development of various power electronics applications facilitates the integration of many smart grid applications in recent years. However, integration of intermittent renewable energy sources, highly stochastic electric vehicles (EVs) activities on the grid and time-varying smart loads have increased the level of grid vulnerability to unusual and high complexity and quality-related problems. Among these problems is to accurately estimate the real contribution and consumption of household loads, in the era of smart appliances and interoperability operation, and its relative impact to the grid's operation. Specifically, household loads represent a significant percentage of electrical energy consumption and therefore, could offer great prosperity to the rise of the demandside management (DSM) programs which subsequently improve the stability of the grid's operation. As a result, our main focus in this dissertation is to develop DSM strategies based on Artificial Intelligence (AI) techniques to properly model and estimate the amount of support smart homes could offer to the smart grids and microgrid's operation. 
Throughout the way to achieve our goals, we develop an energy management framework for smart homes that operate in efficient and reliable microgrids with multiple energy sources and energy storage applications to meet the demands at stable voltage and frequency limits. Furthermore, we develop a precise short-term load forecasting (STLF) which is a critical tool needed to manage DSM program for residential loads that have very high uncertainty and volatility in load consumption. We also develop an energy exchange portal with communication sources, demands and connectivity information between each consumer and the local power utility at the distribution level. Finally, creative AI methodologies have been developed throughout the way to facilitate the integration, control and management of the DSM programs taking into account the consumers' own privacy and security. The security of the DSM is provided by preserving the indoor privacy of the smart homes by sharing a limited and encoded data among household appliances controllers. 


\section{TABLE OF CONTENTS}

CHAPTER

PAGE

Chapter1 Introduction and literature review

$1.1 \quad$ Literature Review

1

1.1 Literature Review

1.2 Problem Statement

1.3 Research Objectives

1.4 Original Contribution of this Dissertation

1.5 Dissertation Organization

Chapter 2 Design and Control of Hybrid AC/DC Microgrid for Future Smart Grid

$2.1 \quad$ Introduction

2.2 system description, Modeling, and Control

2.2.1 PV System Model and Interface

2.2.2 Battery Converter Model and Control

2.2.3 Bi-directional DC/AC converter model and control

2.3 CONTROL PARAMETERS DESIGN USING GA.

$2.4 \quad$ SIMULATION RESULTS

$2.5 \quad$ CONCLUSION

Chapter 3 Non-Intrusive Load Monitoring (NILM) Techniques for Load Forecasting 36

3.1 Introduction

3.2 Non-Intrusive load monitoring

3.2.1 Dataset and Non-Intrusive Load Monitoring Tool Kit (NILMTK). 
3.2.3 Load disaggregation 48

3.3 The implemented Short-Term Load Forecasting 50

$\begin{array}{lll}3.4 & \text { Simulation result } & 50\end{array}$

$\begin{array}{lll}3.5 & \text { CONCLUSION } & 51\end{array}$

Chapter 4 Evaluation of NILM-Based Deep Learning Techniques for Efficient Demand Side Management $\quad 52$

$\begin{array}{lll}4.1 & \text { Introduction } & 53\end{array}$

4.2 ENERGY DISAGGREGATION 57

4.2.1 Non-Intrusive Load Monitoring Tool Kit (NILMTK) 58

4.2.2 Energy Disaggregation Algorithms for Home Consumption. 59

4.2.2.1 Denoising Autoencoder (DAE) 59

4.2.2.2 Recurrent Neural Network (RNN) 60

4.2.2.3 Load Regress the start time, the end time, and the mean power 61

$\begin{array}{lll}4.3 & \text { Disaggregation stage analysis }\end{array}$

4.4 Short-Term Load Forecasting Implementation 65

4.5 Simulation Result $\quad 66$

$\begin{array}{lll}4.6 & 69\end{array}$

Chapter 5 Precise Short-Term Load Forecasting for Residential Demands Considering Highly Stochastic Energy Consumption $\quad 71$

$\begin{array}{lll}5.1 & 71\end{array}$ 
5.2.1 Data Mining Disaggregation algorithms 76

5.2.1.1 Combinatorial Optimization (CO) 77

5.2.1.2 Factorial Hidden Markov Model (FHMM) 79

5.2.1.3 Denoising Autoencoder (DAE) 83

5.2.1.3 Regress start time, end time, and average power (RECTANGLES) 85

5.2.1.3 Recurrent Neural Network (RNN or LSTM) 86

$\begin{array}{lll}5.3 & \text { Disaggregation stage analysis } & 88\end{array}$

5.4 The implemented Short-Term Load Forecasting 92

$\begin{array}{lll}5.5 & \text { Simulation Results } & 92\end{array}$

$\begin{array}{lll}5.6 & \text { Conclusion } & 95\end{array}$

Chapter 6 Power Quality Enhancement of Low Voltage Distribution Grid 96

$6.1 \quad$ Introduction 96

6.2 Proposed system description and the control technique 100

$\begin{array}{ll}\text { 6.2.1 The proposed system description } & 100\end{array}$

$\begin{array}{ll}\text { 6.2.2 The control technique } & 101\end{array}$

$\begin{array}{lll}6.3 & \text { Results and Discussion } & 103\end{array}$

$\begin{array}{lll}\text { 6.3.1 Case study A } & 103\end{array}$

$\begin{array}{ll}\text { 6.3.2 Case study B } & 107\end{array}$

6.4 Hardware Implementation \& Experimental Results $\quad 110$ 
Chapter 7 An Optimal Energy Management System for Real-Time Operation of Multiagent-Based Microgrids

$7.1 \quad$ Introduction

7.1.1 Target

7.1.2 System Component

7.1.3 Method

7.2 Problem Formulation

7.3 Proposed Algorithm for the Real-Time Optimization

7.4 Proposed Multiagent EMS Platform

7.4.1 Management Agents

7.4.2 Backup Agent

7.4.3 Interoperability Agents

7.5 Results

7.5.1 Description of the Cyber-Physical Setup

7.5.2 Experiment Results

7.5.2.1 Case Study 1

7.5.2.2 Case Study 2

7.5.2.3 Case Study 3 
7.7 Conclusions

Chapter 8 Medium Voltage DC Testbed: An Essential Tool to Facilitate the Futuristic integration of DC Microgrids/Nanogrids

$8.1 \quad$ Introduction

8.2 Construction of PET I

8.3 Construction and Control of PET II

8.3.1 Five-level Neutral-Point-Clamped Square-wave Inverter

8.3.2 Three-phase matrix Converter

8.3.3 The programmable load operation of PET II

$8.4 \quad$ Simulation Results

8.5 Conclusion

172

Chapter 9 Fault Detection and Compensation for a PV System Grid Tie Inverter

9.1 Introduction

9.2 System description

9.2.1 PV Array

9.2.2 DC-DC converter

9.2.3 DC bus

9.2.4 Proposed DC - AC Converter

9.3 Fault Detection And Isolation

9.4 Reconfiguration technique

9.4.1 Disconnection of the faulty switch 
9.4.2 Connection of the redundancy branch

9.5 Simulation result

9.5.1 Open Circuit Fault

9.5.2 Short Circuit Fault

9.6 Conclusion

189

Chapter 10 EXPERIMENTAL SETUP OF THE MICRO/NANO GRID TO INVESTIGATE THE DEMAND SIDE MANAGEMENT SCHEMES

$10.1 \quad$ Introduction

10.2 Source Components of the Micro/Nano grid

10.2.1 PV Emulator

10.2.2 Programmable DC Power Supply

10.2.3 Synchronous Generator

10.2.4 Energy Storage

10.2.4.1 Lead-Acid battery

10.2.4.2 Lithium-Ion battery

10.2.4.3 Flywheel

10.3 Load Components of the Micro/Nano grid

10.3.1 Fixed step Controllable Loads

10.3.2 Variable step Controllable Loads 
10.4.3 DC/DC Converter 201

10.4.4 DC/AC Converter 202

10.5 Hybrid DC-AC operation and experiment's guideline steps 203

10.5.1 Hybrid DC-AC grid operation 203

10.5.2 Experiment's guideline steps 207

10.5.2.1 AC side operation guide 207

10.5.2.2 DC side operation guide 214

Chapter 11 Conclusions and Recommendations for Future Work 216

11.1 Conclusions 216

11.2 Recommendations for Future Work 220

List of References $\quad 222$

VITA 245 


\section{LIST OF TABLES}

TABLE

PAGE

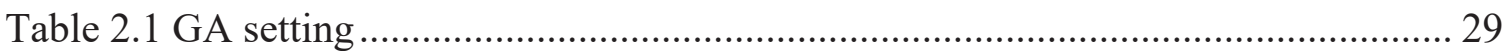

Table 2.2 OPTIMIZED FUZZY PARAMETERS......................................................... 30

Table 3.1 Comparison of the RMSE and NRMSE with the three predictors approach.... 51

Table 4.1 Load Forecasting Performance Comparison for the seen home ........................ 68

Table 4.2 Load Forecasting Performance Comparison for the unseen home ................... 69

Table 5.1 Load Forecasting Performance Comparison for the seen home ....................... 93

Table 5.2 Load Forecasting Performance Comparison for the unseen home ................... 94

Table 6.1 the experimental setup Parameters …………………………….................. 113

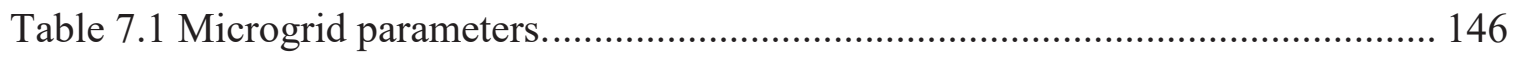

Table 8.1 The HCC Switching Sequence of leg 'a' of the MC ..................................... 167

Table 8.2 MVDC simulation model parameter........................................................... 168

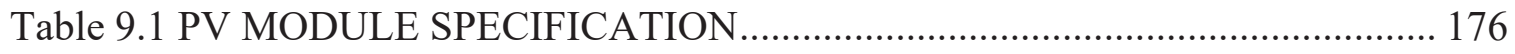

Table 10.1 Synchronous generator parameters............................................................ 197 


\section{LIST OF FIGURES}

FIGURE

PAGE

Figure 2.1 The main configuration for the hybrid AC-DC Microgrid 20

Figure 2.2 The schematic diagram for the PV with the boost converter interface to the DC bus. 22

Figure 2.3 The flow chart for the MPPT method (Perturb \& Observation). ................... 22

Figure 2.4 Block diagram for the boost converter control....................................... 23

Figure 2.5 The schematic diagram for the battery with the bi-directional DC-DC converter interface to the DC bus 24

Figure 2.6 The flow chart for the MPPT method (P\&O) ......................................... 25

Figure 2.7 Schematic diagram of the AC/DC converter................................................ 27

Figure 2.8 GA optimization results (a) Best Fitness, (b) Best Values ............................ 29

Figure 2.9 The schematic diagram for Matlab/Simulink model of the hybrid AC/DC

microgrid 30

Figure 2.10 Active power flow in the DC microgrid (a) Generated power from the PV array (b) Battery power (c) Load power. 32

Figure 2.11 (a) AC bus voltage (RMS value) for phase a (b) AC microgrid frequency (c) DC bus voltage. 33

Figure 2.12 Active power flow in the AC microgrid (a) Synchronous generator output power (b) Inverter power at the PCC(c) Load power ....................................... 34

Figure 3.1 Block diagram for the proposed household load forecasting approach. ........ 42

Figure 3.2 NILMTK's process block diagram. 43 
Figure 3.3 Illustration of a Factorial Hidden Markov Model.

Figure 3.4 Aggregated power for Home (b). Comparison between predicted and ground truth fridge power. (c) Comparison between predicted and ground truth microwave power. (b) Comparison between predicted and ground truth Kitchen Sockets power by using FHMM algorithm for disaggregation. 48

Figure 3.5 (a). Aggregated power for Home (b). Comparison between predicted and ground truth fridge power. (c) Comparison between predicted and ground truth microwave power. (b) Comparison between predicted and ground truth Kitchen Sockets power by using $\mathrm{CO}$ algorithm for disaggregation.

Figure 3.6 Comparison between the actual and predicted power demand with three different algorithms FFANN, FFANN combined with FHMM, and FFANN 49

Figure 3.7 Show the difference Error point by point............................................ 51

Figure 4.1 The proposed household's load forecasting block diagram. ........................ 58

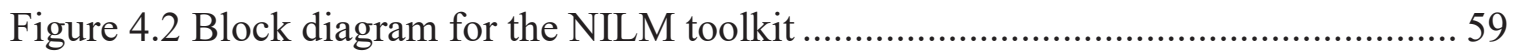

Figure 4.3 Shows the ED output performance by using DAE technique vs the ground truth data. 60

Figure 4.4 Shows the ED output performance by using LSTM technique vs the ground truth data.

Figure 4.5 Shows the ED output performance by using RECTANGLES technique vs the ground truth data. 62

Figure 4.6 NILM performance analysis for home seen during training 64

Figure 4.7 NILM performance analysis for home unseen during training 65 
Figure 4.8 The actual and predicted load demand by different method for the home whose historical data was seen during the NILM training.

Figure 4.9 The actual and predicted load demand by different method for the home whose historical data was unseen during the NILM training. 67

Figure 5.1 Block diagram for the proposed household load forecasting approach. 75

Figure 5.2 Shows the energy disaggregation algorithm output by using the $\mathrm{CO}$ technique.

(a) Aggregated power consumption for the home; (b) Dish Washer estimated and Ground truth power demand; (c) Fridge estimated and Ground truth power demand;

(d) Kettle estimated and Ground truth power demand; (e) Microwave estimated and Ground truth power demand; (f) Washing machine estimated and Ground truth power demand 77

Figure 5.3 Shows the energy disaggregation algorithm output by using the FHMM technique. (a) Aggregated power consumption for the home; (b) Dish Washer estimated and Ground truth power demand; (c) Fridge estimated and Ground truth power demand; (d) Kettle estimated and Ground truth power demand; (e) Microwave estimated and Ground truth power demand; (f) Washing machine estimated and Ground truth power demand 82

Figure 5.4 Shows the energy disaggregation algorithm output by using the DAE technique. (a) Aggregated power consumption for the home; (b) Dish Washer estimated and Ground truth power demand; (c) Fridge estimated and Ground truth power demand; (d) Kettle estimated and Ground truth power demand; (e) Microwave estimated and Ground truth power demand; (f) Washing machine estimated and Ground truth power demand 84 
Figure 5.5 Shows the energy disaggregation algorithm output by using the DAE technique. (a) Aggregated power consumption for the home; (b) Dish Washer estimated and Ground truth power demand; (c) Fridge estimated and Ground truth power demand; (d) Kettle estimated and Ground truth power demand; (e) Microwave estimated and Ground truth power demand; (f) Washing machine estimated and Ground truth power demand 85

Figure 5.6 Shows the energy disaggregation algorithm output by using RNN or LSTM technique. (a) Aggregated power consumption for the home; (b) Dish Washer estimated and Ground truth power demand; (c) Fridge estimated and Ground truth power demand; (d) Kettle estimated and Ground truth power demand; (e) Microwave estimated and Ground truth power demand; (f) Washing machine estimated and Ground truth power demand 87

Figure 5.7 Energy disaggregation performance analysis for home seen during training . 90 Figure 5.8 Energy disaggregation performance analysis for home unseen during training

Figure 5.9 The actual and forecasted load by different methods for the home seen during the NILM training. 93

Figure 5.10 The actual and forecasted load by a different method for the home was unseen during the ED training 94

Figure 6.1 IEEE 13-bus test feeder system with a smart load 97

Figure 6.2 A block diagram for the proposed control technique 101

Figure 6.3 A comparison between the active, reactive power, and the PU voltage at buses $\mathrm{X}, \mathrm{M}$, and Z, respectively 104 
Figure 6.4 A comparison between three-phase current (RMS) at buses X, M, and Z, respectively, respectively 105

Figure 6.5 The three-phase voltage, load current, converter current, and bus current respectively at $\mathrm{PCC}$. 106

Figure 6.6 Phasor Diagram for the voltage and current at PCC, (a) before the proposed technique (b) after the proposed technique 107

Figure 6.7 Simulation result: (a) DC link bus voltage (V), (b) output power of PV system (Watt), (c) Local DC load power (Watt), (d) The grid-tie converter power (Watt) 108 Figure 6.8 Simulation result: (a) AC load current, (b) Zoom in the AC load current (c) Zoom in for AC load current (d) Zoom in for AC supply current (e) Zoom in for AC supply current (f) AC supply current 109

Figure 6.9 Experimental setup block diagram 111

Figure 6.10 (a) DC link bus voltage (V), (b) the output power of PV system (Watt), (c) AC load current, (d) Zoom in for AC load current (e) Zoom in for AC load current (f) Zoom in for AC load current (g) Zoom in for AC supply current (h) Zoom in for AC supply current (i) Zoom in for AC supply current (j) AC supply current ....... 114

Figure 6.11 (a) Phase a Voltage (V) and load current, (b) Zoom in between (25.03-25.1) sec, (c) Zoom in between (150.03-150.1) sec, (d) Zoom in between (200.03-200.1) sec, (e) Zoom in for between (25.03-25.1) sec, (f) Zoom in between (150.03-150.1) sec, (g) Zoom in between (200.03-200.1) (h) Phase a Voltage (V) and phase a supply current. 115

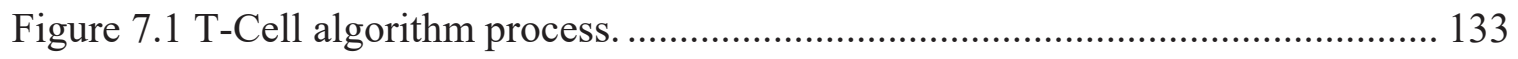

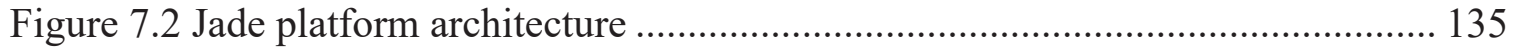


Figure 7.3 MAS architecture.

Figure 7.4 The fault tolerance mechanism in the MAS platform.

Figure 7.5 Interoperability Communication Setup .................................................. 139

Figure 7.6 Generation station hardware and control............................................. 140

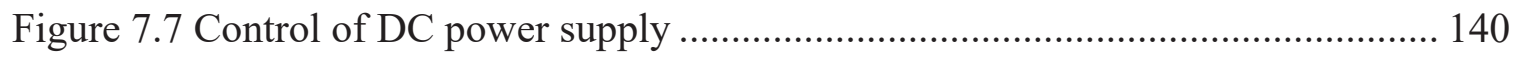

Figure 7.8 SCADA interface in ESRL .............................................................. 142

Figure 7.9 Communication between MGO, MGS and other agents.......................... 143

Figure 7.10 Fault tolerance mechanism in the JADE platform ................................. 145

Figure 7.11 Comparison between simulated output and testbed measured values of wind power.

Figure 7.12 Comparison between simulated output and testbed measured values of PV power

Figure 7.13 Comparison between simulated output and testbed measured values of energy storage power. 148

Figure 7.14 Comparison between simulated output and testbed measured values of load power. 149

Figure 7.15 Comparison between simulated output and testbed measured values of controllable load 150

Figure 7.16 Comparison between simulated output and testbed measured values of grid

power. 150

Figure 7.17 Experimental results of the MG powers during $24 \mathrm{~h}$ operation 151

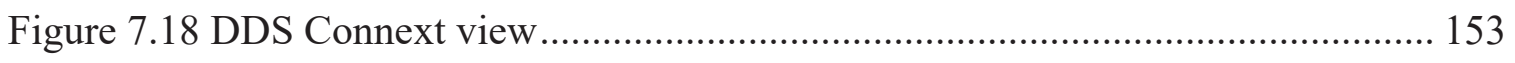

Figure 8.1 The architecture of the proposed MV DC testbed. 161 
Figure 8.2 Controller of the single-phase inverter of PET1 162

Figure 8.3 Switching sequence associated with each voltage level of the 5-level NPC inverter 165

Figure 8.4 The proposed firing circuit of the 5-level NPC inverter. 165

Figure 8.5 The proposed firing circuit of the 5-level NPC inverter 166

Figure 8.6 MV DC bus 1 voltage with different reference levels. 168

Figure 8.7 Injected power from PET2 to the grid (different power levels at a fixed voltage level at MV DC bus2). 169

Figure 8.8 Three-phase currents injected from PET2 to the grid at different power levels

Figure 8.9 Switching pulses of the four switches of the single-phase inverter of PET1 170 Figure 8.10 Injected power from PET2 to the grid (different power levels at a fixed voltage level at MV DC bus2). 171

Figure 9.1 An overall grid-connected PV system with modified inverter topology....... 175

Figure 9.2 The modified inverter topology in the healthy mode ............................... 178

Figure 9.3 Detection and identification block diagram ........................................ 179

Figure 9.4 the modified inverter topology in the post fault mode .............................. 180

Figure 9.5 The behavior of grid-tie inverter topology with switch S1 open circuit ....... 181

Figure 9.6 The behavior of grid-tie inverter topology with switch S4 open circuit ....... 182

Figure 9.7 The behavior of grid-tie inverter topology with switch S3 open circuit ....... 183

Figure 9.8 The behavior of grid-tie inverter topology with switch S6 open circuit ....... 184

Figure 9.9 The behavior of grid-tie inverter topology with switch S1 short circuit ....... 185

Figure 9.10 The behavior of grid-tie inverter topology with switch S4 short circuit ..... 187 
Figure 9.11 The behavior of grid-tie inverter topology with switch S3 short circuit ..... 187

Figure 9.12 The behavior of grid-tie inverter topology with switch S6 short circuit ..... 188

Figure 10.1 Block diagram for the proposed PV Emulator. ....................................... 192

Figure 10.2 GUI of the PV emulator Using Matlab-Control Desk............................. 193

Figure 10.3 PV emulator's Matlab-Simulink model.............................................. 194

Figure 10.4 Programmable DC Power Supply. ......................................................... 196

Figure 10.5 Synchronous generator at the ESRL Smart Grid Testbed........................ 196

Figure 10.6 Battery Bank Stored Safely in a Closed Cabinet..................................... 198

Figure 10.7 Lithium-ion battery bank module....................................................... 199

Figure 10.8 Configuration of the variable step controllable loads............................. 200

Figure 10.9 Transmission Line models.............................................................. 201

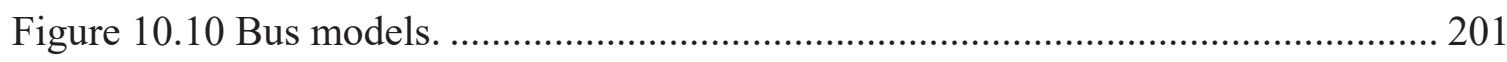

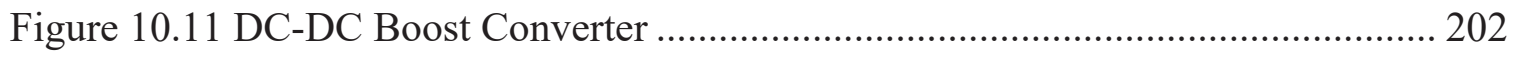

Figure 10.12 The DC/AC IGBT-based converter.................................................. 203

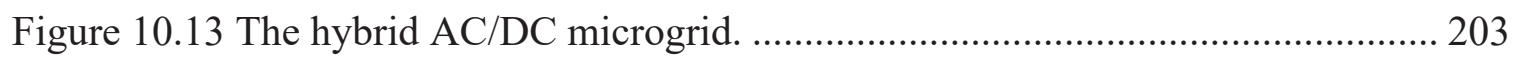

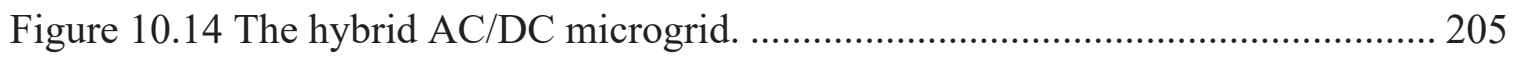

Figure 10.15 Hybrid microgrid operation performance under the severe condition: a)

Correspodnet system frequency, b) power received at the microgrid. .................. 206 


\section{LIST OF ACRONYMS}

\section{ACRONYMS DETAILS}

AES

AGC

AIMD

BESS

BEV

COP

CPP

DA

DDS

DG

DSM

DSO

EMALS

ERCOT

ESS

EV

EVA

FLC

FR

G2V
All Electric Ship

Automatic Generation Control

Additive Increase - Multiplicative Decrease

Battery Energy Storage Station

Battery Electric Vehicle

Conference of the Parties

Critical Peaking Pricing

Day-Ahead

Data Distribution Service

Distributed Generation

Demand-Side Management

Distribution System Operator

Electromagnetic Aircraft Launch System

Electric Reliability Council of Texas

Energy Storage Systems

Electric Vehicle

Electric Vehicle Aggregator

Fuzzy Logic Controller

Frequency Regulation

Grid to Vehicle 


\begin{tabular}{|c|c|}
\hline $\mathrm{HEV}$ & Hybrid Electric Vehicle \\
\hline ICE & Internal Combustion Engine \\
\hline IEC & International Electrotechnical Commission \\
\hline LA & Load Aggregator \\
\hline LFC & Load Frequency Control \\
\hline LSE & Load-Serving-Entity \\
\hline LVAC & Low Voltage Alternative Current \\
\hline LVDC & Low Voltage Direct Current \\
\hline MMGA & Mathematical Morphology Gradient Algorithm \\
\hline MTOU & Multi-Group Time-of-Use \\
\hline MTOUCP & Multi-Group TOU with Critical Peaking \\
\hline MOOP & Multi-Objective Optimization Problem \\
\hline MVAC & Medium Voltage Alternative Current \\
\hline MVDC & Medium Voltage Direct Current \\
\hline NSGA & Non-dominated Sorting Genetic Algorithm \\
\hline PEV & Plug-in Electric Vehicle \\
\hline $\mathrm{PF}$ & Pareto Front \\
\hline PHEV & Plug-in Hybrid Electric Vehicle \\
\hline PL & Parking Lot \\
\hline $\mathrm{PO}$ & Pareto Optimality \\
\hline POC & Point of Charging \\
\hline PV & Photo-Voltaic \\
\hline
\end{tabular}




$\begin{array}{ll}\text { PWM } & \text { Pulse Width Modulation } \\ \text { RT } & \text { Real-Time } \\ \text { SOC } & \text { State of Charge } \\ \text { TOU } & \text { Time-of-Use } \\ \text { V2B } & \text { Vehicle to Building } \\ \text { V2G } & \text { Vehicle to Grid } \\ \text { V2V } & \text { Vehicle to Vehicle }\end{array}$




\section{Chapter 1 Introduction and literature review}

Demand-side management has a vital role to support the continually increasing rise in smart grid applications. New implementations of innovative techniques on various power system infrastructure levels facilitate the integration of new ideas to improve system operations. The integration of smart grid architectures and concepts could add extra burdens on the grid, which increase in both complexity and uncertainty. In addition, the increased penetration of intermittent renewable energy sources, electric vehicles (EVs), and time-varying loads at the distribution system level make the grid vulnerable to other stochastic and challenging issues. This makes utility-customer interactions more difficult

and even sometimes undesirable. Household loads represent a significant percentage of electrical energy consumption. Therefore, active consumer participation by means of households' demand-side response (DSR) lead to better operation and control at the grid level and enhance both the power system stability, security and reliability.

\subsection{Literature Review}

The energy infrastructure is being improved with smart technologies and increased utilization of alternative renewable sources. Smart homes and appliances are also on the increase giving rise to additional issues in implementing the new load demands into the grid. Examples could be time-varying loads demand and stochastic electric vehicles (EVs) in the distribution grid; which adds a burden on the grid and threaten its stability. These issues adds a significant intermittent and highly stochastic issues that need to be solved to improve reliability. 
Household load demand represents a significant portion of electrical energy consumption. Households' demand-side response (DSR) enables the active participation of the household load instead of considering it as a passive load. Therefore, it provides a positive impact on power system stability. Forecasting the household load consumption is essential for residential DSR program. Precise short-term load forecasting (STLF) on the household level can significantly influence the management and the power system operations at the distribution level. In addition, STLF is a necessary tool required in the management and control of the households' loads to enable smart consumption. Yet, the main challenge that faces the STLF at this level of the grid is the high percentage of uncertainty in the load demand, affected by customer behavior, which is too difficult to expect. There is a lot of research done in the literature on load forecasting. Most of this work is built upon time-series analysis, for example, exponential smoothing and autoregressive integrated moving average (ARIMA) and machine learning methods, for instance, feed-forward artificial neural networks (FFANN) and support vector machine (SVM). An adopted ARIMA model for a day ahead load forecasting is offered [10]. The prediction mechanism in this study is based on gathering the targeted day with a comparable meteorological day in the historical data. Another study offers Radial basis function (RBF) neural network is used for STLF [1]. A combination of the adaptive neural fuzzy inference system (ANFIS) and RBF neural network is provided in [2]. This study was established to regulate the forecasting by considering the real-time electricity price. A neural network-based predictor for very short-term load forecasting [3]. It considered only the load values of the current and previous time steps as the Input to predict the load value at the following time step. A group of extreme learning machines (ELMs) to acquire and 
predict the total load of the Australian national energy market [4]. A committed input selection strategy to work with the hybrid prediction framework based on wavelet transformation and the Bayesian neural network was presented in [5]. All the previous load-forecasting techniques mentioned in the literature can be concluded in three categories. In the first category, all approaches avoid the uncertainty by Clustering/classification techniques that gather similar customers, days or weather to reduce the alteration of uncertainty within each cluster [6]. However, the performance is comprehensively dependent on the available data. The second category is trying to cancel out the uncertainty by using aggregated smart metering data so that the aggregated load exhibits mostly regular patterns and more easier to predict. In addition, it is used only on an aggregated level. The third category is demanding to separate the regular pattern from the other component of load profile such as uncertainty and noise by pre-processing techniques. Mostly spectral analysis such as empirical mode decomposition (EMD) [7], Fourier transform [8], and wavelet analysis [9]. This method cannot be used in household load forecasting due to the high proportion of uncertainty in the load pattern.

The predicted massive utilization of photovoltaic (PV) systems in the residential sector is justified for several reasons. The efficiency of the PVs is improving, the cost of manufacturing is declining, and the incentives provided by the utility to decrease the utility bills are a fact. In addition, it is highly expected that the DC loads usage is increasing in the distribution network. Such as the usage of new DC appliances and equipment in the residential sector (consumer electronics, LED lighting, and compact fluorescent lamps) [10]. Despite the benefits of the innovations at source and demand sectors, many power 
quality problems are facing the distribution network against all these innovations. For example, the integration of rooftop PVs may cause severe voltage fluctuations and unbalance due to the uncertainty and the lake of availability of the irradiance [11]-[14]. In addition, the increased usage of DC Loads adds more harmonics to the distribution network [15], [16]. That is because all individual DC load needs an individual rectifier to facilitate the connection of the equipment to the current $\mathrm{AC}$ network and provide power factor correction to comply with the utility policy [17], [18]. Moreover, the expedite movement toward the usage of a sensitive component in residential, commercial, industrial, traction applications such as refrigerators, televisions, computers, switched-mode power supplies add nonlinearly, and unbalanced loads to the distribution network [19]. Degrading the power quality of the power system network wears out the network component, increases the probability of system shutdown, and increases the maintenance disbursal [20-22]. Consequently, enhancing the power quality of the electric power network is a critical factor in the process of power generation and delivery.

The electrical regulation standards in distribution networks such as power factor and harmonics levels are applied to all AC loads connected to the current AC network. Some of these regulations define the interconnection requirement of the distributed generation (DG) units in low-voltage distribution systems [23]-[25]. Others, determine the interconnection regulation for the electric loads, such as home appliances [26]-[28]. The main concern for utility companies is the massive utilization of loads in current homes that do not comply with the standard regulation and degrade the power quality of the power system network [29]. 
The IEEE 1547 is one of the power quality standards, which define the harmonics and total harmonic distortion (THD) levels. Several works introduce different ways to mitigate harmonic components and compensate for reactive current, negative harmonics. Some use the injection of negative reactive current to the power network to reduce the harmonics and provide power factor correction [30]-[32]. Another approach allows the simultaneous compensation for the harmonics and reactive power issues called Active Power Filter (APF). In addition, it can provide compensation for the system load unbalance and compensate for voltage fluctuations at the point of common coupling (PCC). The different topology of APF such as series, and shunt. The performance of the APF is depended on the accuracy of selecting a suitable method to extract undesired current. The Synchronous Reference Frame (SRF) method is used in three-phase balanced systems [33]-[35]. Another effort work based on instantaneous power theory in three phases, the unbalanced system [36]-[39].

\subsection{Problem Statement}

The innovations at different power system infrastructures' levels facilitate the integration of new smart grid ideas. However, new architectures of the smart grid add an extra burden on the grid as it relates to complexity and uncertainty. Because of the increased penetration of intermittent renewable energy, highly stochastic electric vehicles (EVs) activities as well as time-varying loads in the distribution system, the grid will be vulnerable to unusual, challenging problems that increase difficulties to achieve better and reliable utility-customer interactions. Household loads represent a significant percentage 
of electrical energy consumption. The households' demand-side response (DSR) enables active participation of these loads in the grid enhancing power system stability.

For several decades, network operators have used various forms of Demand Side Management (DSM) to improve the balancing of system supply and demand and to reduce load peaks. Many of the practical DSM programs implemented worldwide to date have focused on large industrial consumers since these have demand of sufficient volume to produce significant effects at the system level. However, with the introduction of smart metering and time-varying electricity rates for individual customers, new opportunities are being created for small electricity users to participate in demand-side services. Recently, much research has focused on DSM in the residential sector [40]-[47].

Many existing DSM programs use direct load control, where the network operator can directly actuate large industrial loads according to the needs of the network, and the exact terms of the DSM contract are agreed beforehand. While direct load control may be suitable for specific industrial users, it has technical and practical difficulties in the context of residential users, where direct actuation of loads is typically considered an invasion of user privacy and comfort. Also, direct load control may require significant investments to provide additional communication and control technology for each user. Most residential DSM schemes instead rely on the user response to an electricity price signal to produce the required outcome, e.g., [40], [41], [43]-[48].

To mitigate the adverse effect of direct load control on the customer and the power system infrastructure. There will be a need for innovative control and management solutions that result in significant enhancements in the power grid operations. This 
innovative solution will be based on artificial intelligence algorithms that provide more data to efficiently facilitate the deployment of smart homes and Microgrids to the current power system.

\subsection{Research Objectives}

The primary objective of this dissertation is to provide management and control strategies to make the best use of demand-side management in the power systems. The management and control should satisfy the customer or the operator preferences, the system technical constraints, and the involved economic issues. In this paradigm, the deployment of smart homes which allows active load participation are appropriate solutions that can reduce the drawbacks of the massive penetration of renewable energy, Electric Vehicles (EVs), and time-varying loads in the distribution system.

This study is critical because it will provide valuable solutions for many issues that are related to the active participation of the load consumption sector in power grids stability. The study proposes an effective energy management framework and control algorithm that can lead to the optimal control and operation of individual customers in the distribution level and thus bringing benefits to the environment, customers, and system operators.

The development of the current power system grid is essential to serve the client better. Especially, with the massive expansion in the distribution network and the increase of renewable energy penetration. This development means the grid must be reliable, more flexible, and smarter. Households represent a massive portion of the grid infrastructure. The deployment of smart appliances enables the grid to be smarter through energy/demandside management based appliances control. Therefore, Short-Term Load Forecasting 
(STLF) is highly needed to help the energy management for households' systems. STLF at this level of the grid is very challenging due to the high percentage of uncertainty in the load demand.

Based on that, the major aspects of the dissertation are:

1. Developing Demand-side management (DSM) strategies that allow the active participation of smart homes to the power system stability.

2. Developing an energy management framework for Microgrids and smart homes with multiple sources and energy storage to meet the load demand without violating the voltage and frequency constraints.

3. Developing a precise short-term load forecasting (STLF) which is a critical tool needed for the DSM program especially for residential loads that have very high uncertainty and volatility in load consumption.

4. Developing creative techniques based on artificial intelligence algorithms to facilitate DSM programs considering customers' privacy and security.

\subsection{Original Contribution of this Dissertation}

The main goal of the research work in this dissertation is to provide management and control strategies to make the best use of demand-side management in the power systems. The management and control should satisfy the customer or the operator preferences, the system technical constraints, and the involved economic issues. In this paradigm, the deployment of smart homes which allows active load participation are appropriate solutions that can reduce the drawbacks of the massive integration of renewable and distributed energy resources, electric vehicles (EVs), and time-varying loads. 
As we mentioned early, recent advances and development of different fields related to the power system's operation, utilization and control requires much attention for successful implementation of the concept of the smart grids. Among those options set to be one of the promised solution for a smarter grid is demand response management programs which focuses on household loads management of what is considered significant percentage of electrical energy consumption. The households' demand-side response (DSR) enables active participation of these loads in the grid enhancing power system stability. Consequently, the forecasting of household energy consumption is crucial for household DSR programs. Precise short-term load forecasting (STLF) has a significant effect on the accuracy of the household DSR. However, STLF is challenging at this level of the grid due to uncertainty and volatility in load consumption originating from customer behavior, which is too stochastic to predict. The contribution of this dissertation can be divided into:

1- We developed an innovative methodology to enhance household demand forecasting based on energy disaggregation for Short Term Load Forecasting [49]. This approach is constructed from Feed-Forward Artificial Neural Network forecaster and a pre-processing stage of energy disaggregation. This disaggregation technique extracts the individual appliances' load demand profile from the aggregated household load demand to increase the training data window for the proposed forecaster [50], [51]. These proposed algorithms include two benchmark disaggregation algorithms; Factorial Hidden Markov Model (FHMM), Combinatorial Optimization in addition to three adopted Deep Neural Network; long short- term memory (LSTM), Denoising Autoencoder, and a network which 
regress start time, end time, and average power. The proposed load forecasting approach outperformed the currently available state-of-the-art techniques; namely, root mean square error (RMSE), normalized root mean square error (NRMSE), and mean absolute error (MAE).

2- We developed a real-time operation of the energy management system (RT-EMS) is one of the vital functions of Microgrids (MG). In this context, the reliability and smooth operation should be maintained in real-time regardless of load and generation variations and without losing the optimum operation cost. I developed a real-time operation energy management system (RT-EMS) at the residential Microgrid level [52]. This work presents a design and implementation of an RTEMS based on Multiagent system (MAS) and the fast converging developed algorithm to minimize the MG operational cost and maximize the real-time response in grid-connected MG. The RT-EMS has the main function to ensure the energy dispatch between the distributed generation (DG) units that consist of this work of a wind generator, solar energy, energy storage units, controllable loads and the main grid. The MAS has features such as peer-to-peer communication capability, a fault-tolerance structure, and high flexibility, which make it convenient for the MG context. Each component of the MG has its own managing agent. While, the MG operator (MGO) is the agent responsible for running to ensure the seamless operation of the MG in real-time, the MG supervisor (MGS) is the agent that intercepts sudden high load variations and computes the new operating point. 
3- We developed a RT-EMS develops integration of the MAS platform with the Data Distribution Service (DDS) as a middleware to communicate with the physical units. In this work, the proposed algorithm minimizes the cost function of the MG as well as maximizes the use of renewable energy generation; then, it assigns the power reference to each DG of the MG. To verify the performance of the proposed system, an experimental validation in an MG testbed was conducted. Results show the reliability and effectiveness of the proposed multiagent based RT-EMS. Various scenarios were tested such as normal operation as well as sudden load variation.

4- The microgrids offer an essential solution to enable a resilient grid infrastructure since they can continue operating in case of a utility outage. The futuristic distribution system, which is one of the fundamental smart grid concepts, can be viewed as a cluster of microgrids and each microgrid can be viewed as a cluster of distributed energy resources DERs. Microgrids mostly depend on intermittent renewable resources. This configuration may introduce severe stability problems, especially during the islanded operation. Therefore, We developed a hybrid residential microgrid system for future city houses [53]. It is composed of both AC and DC networks tied together through an interlinking bidirectional AC/DC converter (IC). The DC network contains the DC loads and the distributed energy sources (DES) such as Photovoltaic (PV) and battery storage. The AC network includes the AC loads and a synchronous AC generator as the only AC source in islanding operation in this system. The islanding operation at this level of the grid, prone the network to be more vulnerable to stability issues. Therefore, it is essential 
to obtain the best control parameters for the IC controller, which ensures sufficient power exchange and stable operation between the DC/AC networks. Consequently, a Genetic Algorithm (GA) has been used to tune the vector decoupling control parameters of the IC controller to find the best parameter combination to improve the hybrid grid performance. The proposed scheme maintains system stability during various conditions such as variable or pulsed demand and uncertain renewable power sharing. The tuned vector decoupling controlled sinusoidal pulse width modulation (SPWM) technique has been used to not only allow the IC to maintain stable voltage and frequency but also tracks the active and reactive power set points.

5- We developed a control methodology to facilitate the smart integration of a DC Microgrid to Neighborhood Low Voltage Distribution Network (NLVDN). The DC Microgrid connected to the NLVDN through a three-phase voltage source inverter (VSI) [54]. In which, the VSI works as Distribution Static Compensator (DSTATCOM), and the DC link provides an integration point for the PV and the DC native loads. The proposed technique is capable of compensating reactive power, unbalance and harmonics demanded by three-phase non-linear loads and unbalanced connected to the distribution side improves the power quality [55]. Besides, it is able to prevent the source from being overloaded by providing active power support to the load. 


\subsection{Dissertation Organization}

This dissertation is organized in eleven chapters, including the current chapter, which presents the introduction of this dissertation following the problem statement, research objectives, and the significant contribution.

Chapter 2 discusses the Hybrid AC/CD microgrid that is used to verify the proposed energy management schemes. In which, a genetic algorithm-based hybrid AC/DC microgrid control is proposed to ensure system stability. The coordination control scheme is proposed for the Interlinking Converter (IC) to maintain a stable system operation under various load and resource conditions. The Matlab/Simulink simulation verify the tuned coordinated control system. Different resource conditions and load capacities are tested to validate the control method. The results show that the hybrid grid can operate steadily in islanding mode. Stable AC and DC bus voltage can be guaranteed when the operating conditions or load capacities change. The power is smoothly transferred when the load condition varies.

Chapter 3 provides an overview of extracting new features from load pattern for supporting the management system based on a Non-Intrusive Load Monitoring (NILM) techniques. The NILM techniques extract the individual load pattern from the available historical aggregated load demand. These new features increase the training data window for the ANN forecaster and achieve a significant enhancement for its prediction performance. 
Chapter 4 introduces a comparison between the NILM based Deep learning techniques to select the suitable for smart home applications. Three deep neural network architectures have been used for energy disaggregation. 1) denoising autoencoder (DAE); 2) a unique type of a recurrent neural network (RNN) entitled long short- term memory (LSTM); 3) a network that provides rectangles for the estimated demand by regression the start time, end time and average power demand (nicknamed as RECTANGLES). The proposed method outperforms the state of the art techniques in household load forecasting regarding RMSE, NRMSE, and MAE.

Chapter 5 proposes a precise short-term load forecasting for residential loads with high uncertainty in load consumption. An innovative methodology to enhance household demand forecasting based on energy disaggregation for Short Term Load Forecasting. This approach is constructed from Feed-Forward Artificial Neural Network forecaster and a pre-processing stage of energy disaggregation. This disaggregation technique extracts the individual appliances' load demand profile from the aggregated household load demand to increase the training data window for the proposed forecaster. These proposed algorithms include two benchmark disaggregation algorithms; Factorial Hidden Markov Model (FHMM), Combinatorial Optimization in addition to three adopted Deep Neural Network; long short- term memory (LSTM), Denoising Autoencoder, and a network which regress start time, end time, and average power. The proposed load forecasting approach outperformed the currently available state of the art techniques; namely, root mean square error (RMSE), normalized root mean square error (NRMSE), and mean absolute error (MAE). 
Chapter 6 presents a demand response technique to enhance the power quality of the distribution network. This work demonstrates a smart integration of a DC Microgrid to Neighborhood Low Voltage Distribution Network (NLVDN). The DC Microgrid connected to the NLVDN through a three-phase voltage source inverter (VSI). In which, the VSI works as Distribution Static Compensator (DSTATCOM), and the DC link provides an integration point for the PV and the DC native loads. The proposed technique is able to compensate for unbalance, reactive power and harmonics demanded by threephase and non-linear loads and unbalanced connected to the distribution side, leading to the power quality enhancement. Besides, it is able to prevent the source from getting overloaded by providing active power support to the load.

Chapter 7 provides a demand response technique to ensure stability in the distribution network. This work is constructed based on a multiagent real-time operation of the energy management system (RT-EMS) structure. The RT-EMS has the main function to ensure the energy dispatch between the distributed generation (DG) units that consist of this work. Such as a wind generator, solar energy, energy storage units, controllable loads and the main grid. The Multiagent System (MAS) has features such as peer-to-peer communication capability, a fault-tolerance structure, and high flexibility, which make it convenient for the MG context. In addition, the proposed RT-EMS develops integration of the MAS platform with the Data Distribution Service (DDS) as a middleware to communicate with the physical units.

Chapter 8 provides a laboratory equipment to validate the hardware developed to work on the MV levels. This work presents a design of a scaled-down MV DC laboratory testbed. The proposed system is based on Power Electronic Transformers (PETs) in order to provide 
the high step-up ratio of voltage without occupying much space. This MV DC testbed is a laboratory setup that provides a power source and a power sink for any DC hardware to be tested in the MV range. The proposed system is evaluated through Matlab Simulink simulations.

Chapter 9 provides a fault detection and fault-tolerant control mechanism to maintain the continuous operation of the grid-tied inverter, which is commonly used to interface renewable energy and distributed generation to the grid. The fault tolerance is provided using the modified topology of the DC-AC power converter with a redundant standby branch. The proposed system ensures continuous operation without isolating the energy source from the grid during faults on the inverter switch. The fault may be an open circuit fault or short circuit fault.

Chapter 10 provides the experimental work that used to test and validate the demand side management methodologies at the distribution network level. This work will include the design and implementation for different Hardware setup such as the hybrid AC/DC microgrid.

Chapter 11 concludes this dissertation and gives insight into future work. 


\section{Chapter 2 Design and Control of Hybrid AC/DC Microgrid for Future Smart Grid}

In this work, a hybrid residential microgrid system was considered for future city houses. It is composed of both $\mathrm{AC}$ and $\mathrm{DC}$ networks tied together through an interlinking bidirectional AC/DC converter (IC). The DC network contains the DC loads and the distributed energy sources (DES) such as Photovoltaic (PV) and battery storage. The AC network includes the AC loads and a synchronous AC generator as the only AC source in islanding operation in this system.

The islanding operation at this level of the grid, prone the network to be more vulnerable to stability issues. Therefore, it is essential to obtain the best control parameters for the IC controller, which ensures sufficient power exchange and stable operation between the DC/AC networks. Consequently, a Genetic Algorithm (GA) has been used to tune the vector decoupling control parameters of the IC controller to find the best parameter combination to improve the hybrid grid performance.

The proposed scheme maintains system stability during various conditions such as variable or pulsed demand and uncertain renewable power-sharing. The tuned vector decoupling controlled sinusoidal pulse width modulation (SPWM) technique has been used to not only allow the IC to maintain stable voltage and frequency but also tracks the active and reactive power set points. 


\subsection{Introduction}

The microgrids offer an essential solution to enable a resilient grid infrastructure since they can continue operating in case of a utility outage [56]. The futuristic distribution system, which is one of the fundamental smart grid concepts, can be viewed as a cluster of microgrids [57] and each microgrid can be viewed as a cluster of distributed energy resources DERs, which has a connection to the main utility grid. Microgrids could be composed of conventional or renewable DERs. Microgrids mostly depend on intermittent renewable resources. This configuration may introduce severe stability problems, especially during the islanded operation [58], [59]. Minor stability problems can cause cascading outages which would result in a large-scale blackout if proper action is not taken on time. An efficient way to prevent such risks, ensure robustness, and resilience is to operate the grid in a coordinated mode [60], [61] with considering the different parameters of the heterogeneous mix of the available resources. The microgrids are categorized as AC and DC. There's a trend to consider DC microgrids widely. The reasons for this, are well discussed in the literature [62]-[68]. On the other side, the advantages of AC systems cannot be omitted; this is mainly due to the efficient and cheap means of generation provided by the synchronous AC machines. The majority of the loads have been designed to operate in $\mathrm{AC}$ form [66]. Thus, a combination of $\mathrm{AC}$ and $\mathrm{DC}$ microgrids can be introduced as a viable solution.

Photovoltaic (PV) generation is one of the most viable renewable energy sources to be considered in the future smart grid. However, substantial impediments need to be overcome to achieve the more comprehensive utilization of PV. The most salient one is the 
intermittency of PV generation which can be compensated not only by using energy storage technologies but also by demand-side management and exchanges with other power networks: the main grid and surrounding microgrids [67]. Many researchers verify the validation of applying stochastic optimization techniques like a genetic algorithm in tuning the controller parameters [73]-[74]. In [75], authors have an effort to optimize the PID control parameters by selecting best suitable combination to verify the stable control region, and it is applied to the DC-DC converter. Another paper [76] discusses many efforts that done to design non-isolated DC-DC converters for PV applications.

In this work, a Genetic Algorithm (GA) has been used to tune the vector decoupling control parameters of the IC controller to find the best parameter combination to improve the hybrid grid performance. The proposed technique not only guarantees stability in DC side but also coordinates with $\mathrm{AC}$ side for optimum behaviors of the two sides under unpredicted severe changes in both types of demand. The results ensure that the proposed technique improves and maintains stable system operation under various load and resource conditions.

\section{2 system description, Modeling, and Control}

This system consists of a hybrid AC-DC microgrid interconnected to each other with a bi-directional AC-DC converter as shown in Figure 2.1. The DC microgrid depends mainly on renewable energy sources such as PV array to supply its local DC loads. Also, it includes backup battery storage that can support the load deficiencies and the PV source intermittency. The AC microgrid depends mainly on a synchronous generator to supply its 
local AC loads. The AC grid is isolated from the utility to represent microgrid in islanding operation or rural area network.

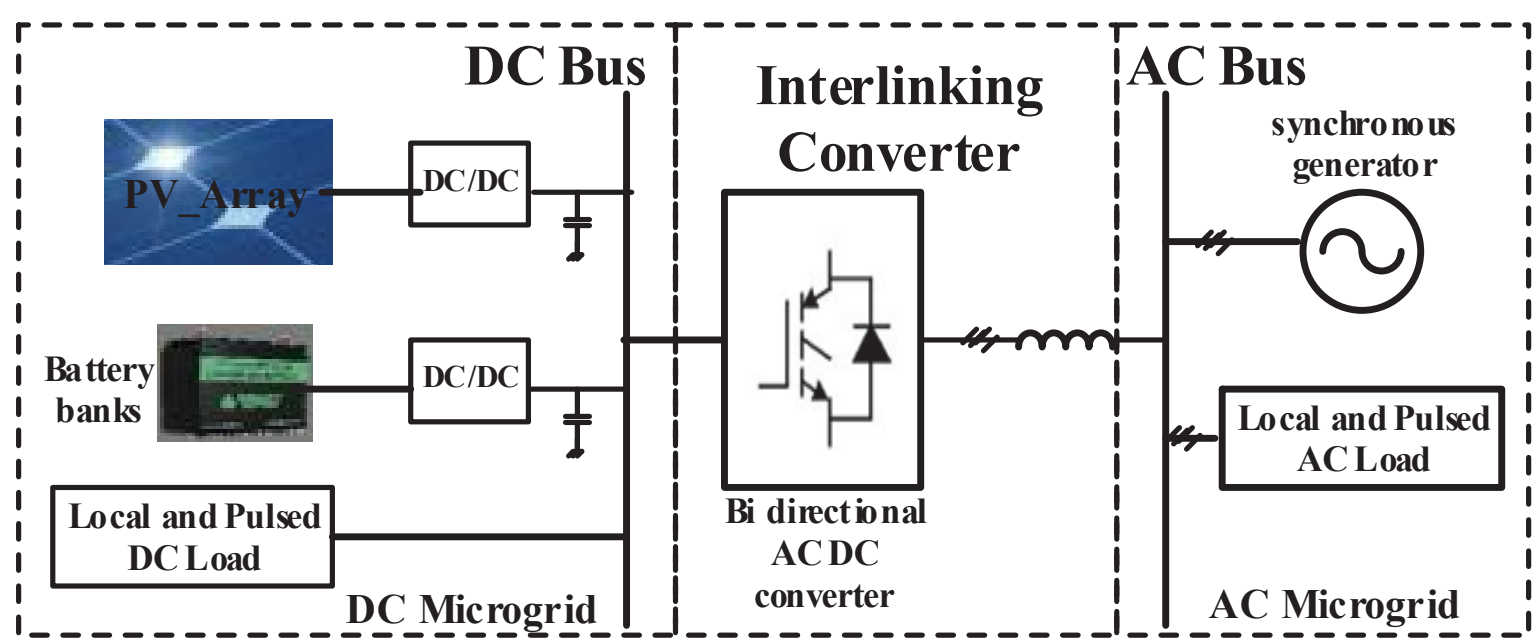

Figure 2.1 The main configuration for the hybrid AC-DC Microgrid

The system has been designed in a way that can autonomously satisfy the power needs in both $\mathrm{AC}$ and $\mathrm{DC}$ microgrid.

\subsubsection{PV System Model and Interface}

A typical $20(\mathrm{~kW})$ maximum output $\mathrm{PV}$ array power is constructed from a number of series and parallel connected modules. It consists of 22 parallel string with 3 series modules each. The commercial SunPower SPR-305-WHT PV 305 W module is considered in this design. The IV characteristic of the PV array is represented by the single diode model which offers both the advantage of accuracy and simplicity [70]-[72]. The PV array current is described by (1).

$$
I_{p v}=I_{L}-I_{S} *\left[\exp \left(\frac{q\left(V_{p v}+I_{P V} * R_{S}\right)}{K_{B} * T * A}\right)-1\right]-\frac{\left(V_{p v}+I_{p v} * R_{S}\right)}{R_{S h}}
$$


Where Rs is the series resistance, and Rsh is parallel leakage resistance, $I_{L}$ is the internal $\mathrm{PV}$ current in the PV array, $I_{p v}$ is the $\mathrm{PV}$ array output current, $I_{S}$ is the reverse saturation or leakage current of the diode. A is the ideality factor of the solar cell. $q$ is the charge of the electron $\left(1.6 \times 10^{-19} \mathrm{C}\right), K_{B}$ is the Boltzmann constant $\left(1.3806488 \times 10^{-23} \mathrm{~J} / \mathrm{K}\right)$.

A DC to DC boost converter is utilized. Figure 2.2 shows the schematic diagram of the PV array, the boost converter, and the DC bus.

This converter has two principal targets: first, step up the PV voltage to the DC bus level and achieve MPPT. Second, to extract as much power as possible from PV panel by performing maximum power point tracking (MPPT).

In this work perturbation and observe $(\mathrm{P} \& \mathrm{O})$ traction algorithm is used to keep track the maximum power even if temperature or irradiance change does occur. The algorithm receives the reading of the $\mathrm{PV}$ output current and voltage to calculate the power. The value of the power at the $k_{t h}$ iteration $(\mathrm{Pk})$ is stored, then the same value is measured and calculated for the $(\mathrm{k}+1)^{\text {th }}$ iteration $(\mathrm{Pk}+1)$.

The power difference between the two iterations $(\Delta \mathrm{P})$ is determined. The converter should decrease the PV panel output voltage if $\Delta \mathrm{P}$ is negative and increase the PV panel output voltage if $\Delta \mathrm{P}$ is positive, which finally leads to adjusting the duty cycle. When $\Delta \mathrm{P}$ is approximately zero, the PV panel reaches the maximum power point. The flow chart of the Perturb and Observe MPPT algorithm is given in Figure 2.3.

The control technique for the boost converter designed based on equations (2), (3) and (4). Where $\mathrm{d} 1$ is the duty cycle ratio of the switch Spv. 


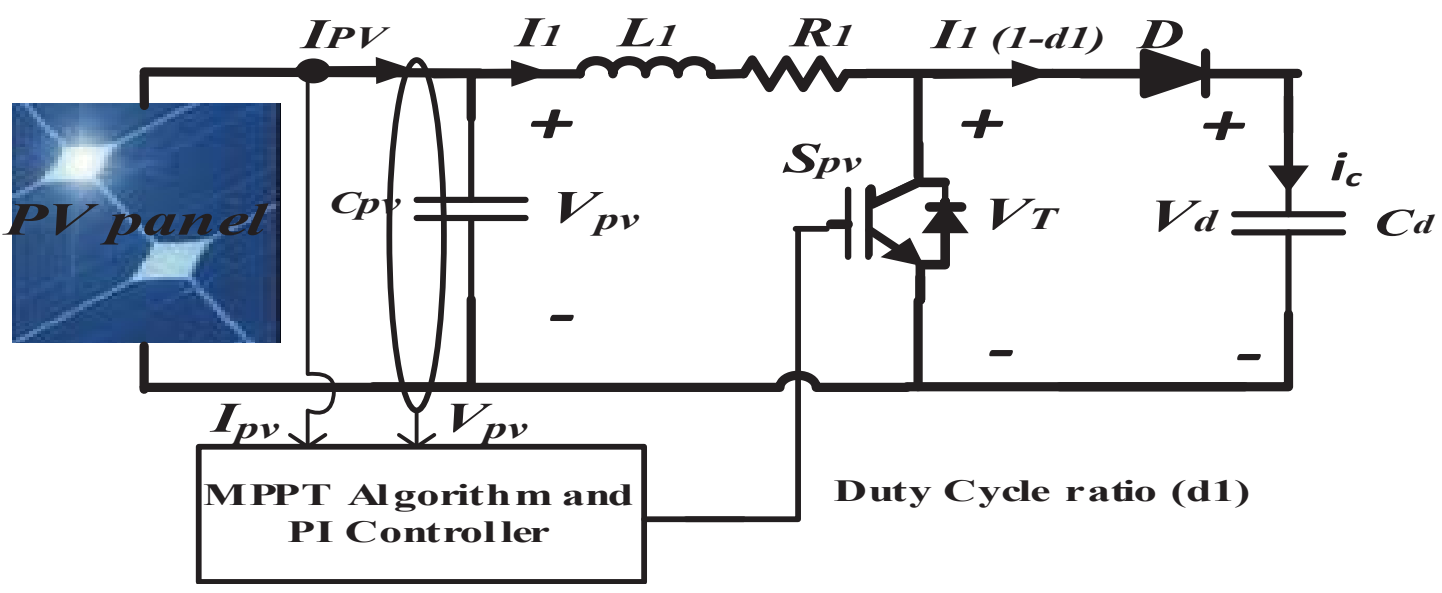

Figure 2.2 The schematic diagram for the PV with the boost converter interface to the DC bus

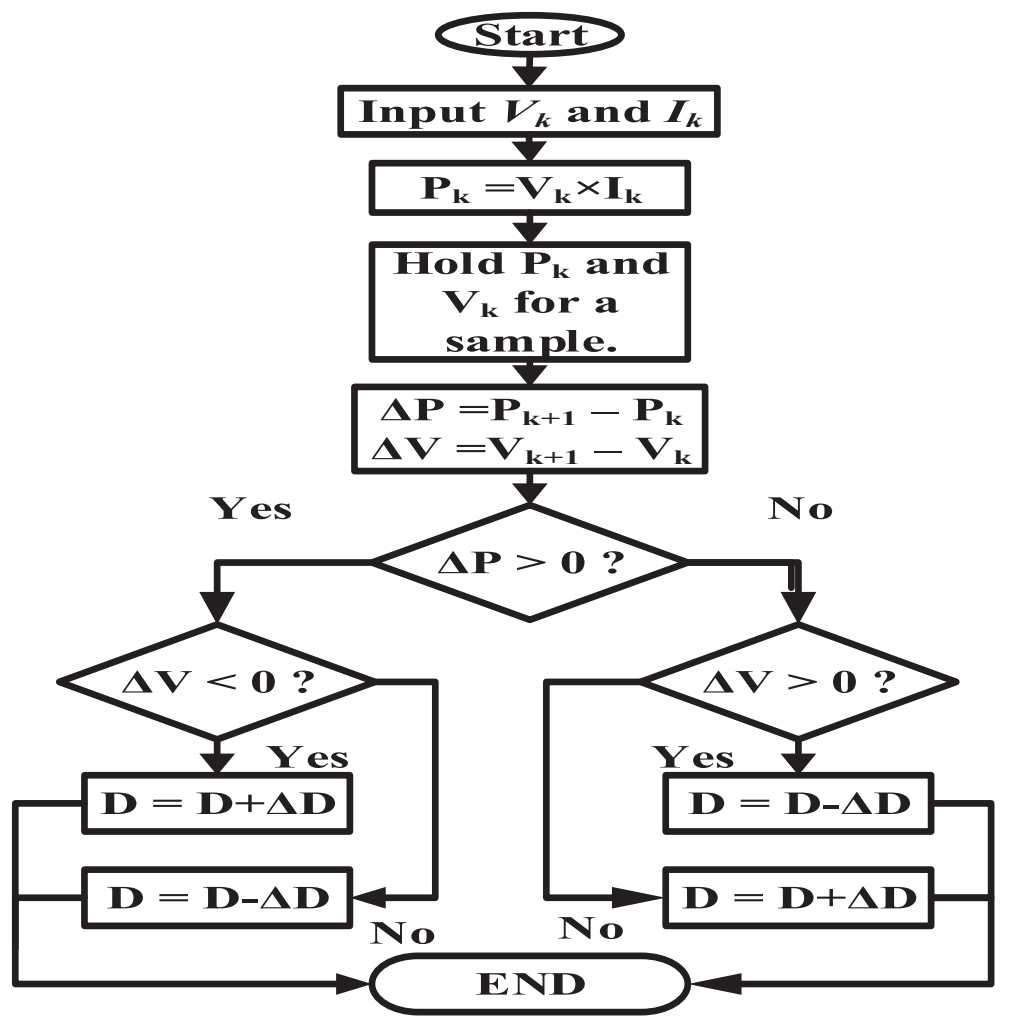

Figure 2.3 The flow chart for the MPPT method (Perturb \& Observation).

The block diagram shown in Figure 2.4 indicates the implementation of the boost converter control algorithm. 
$V_{p v}-V_{T}=L_{1} \frac{d I_{1}}{d t}+R_{1} I_{1}$

$I_{p v}-I_{1}=C_{p v} \frac{d V_{p v}}{d t}$

$V_{T}=V_{d}\left(1-d_{1}\right)$

In which the voltage reference ${ }_{p v}^{*}$ calculated from the P\&O algorithm which mainly depends upon solar irradiation and temperature of PV array. The control composed of dualloop control. The outer voltage loop helps in tracking the reference voltage with zero steady-state error, and inner current loop helps in the improvisation of dynamic response.

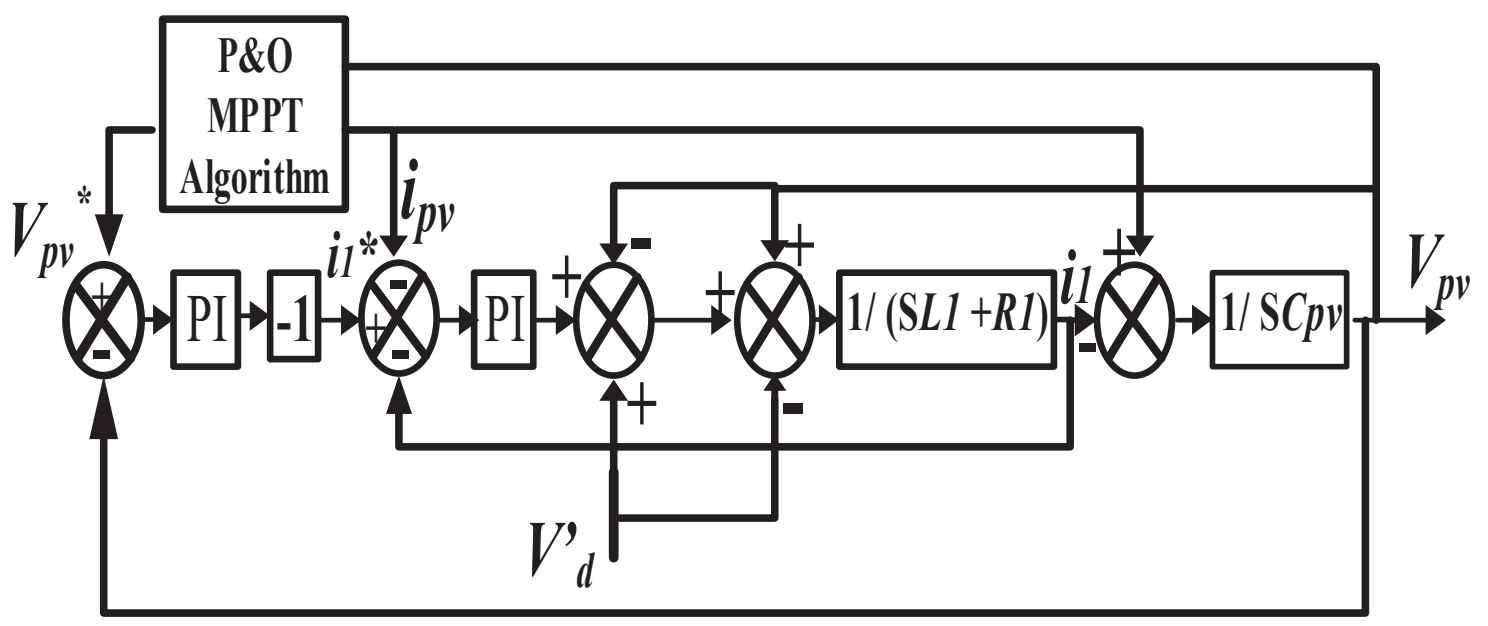

Figure 2.4 Block diagram for the boost converter control.

\subsubsection{Battery Converter Model and Control}

The battery converter topology is a bidirectional DC-DC converter as shown in Fig.2.5. The battery is connected to the low voltage side, while the DC bus is connected to the high voltage side of the converter. 


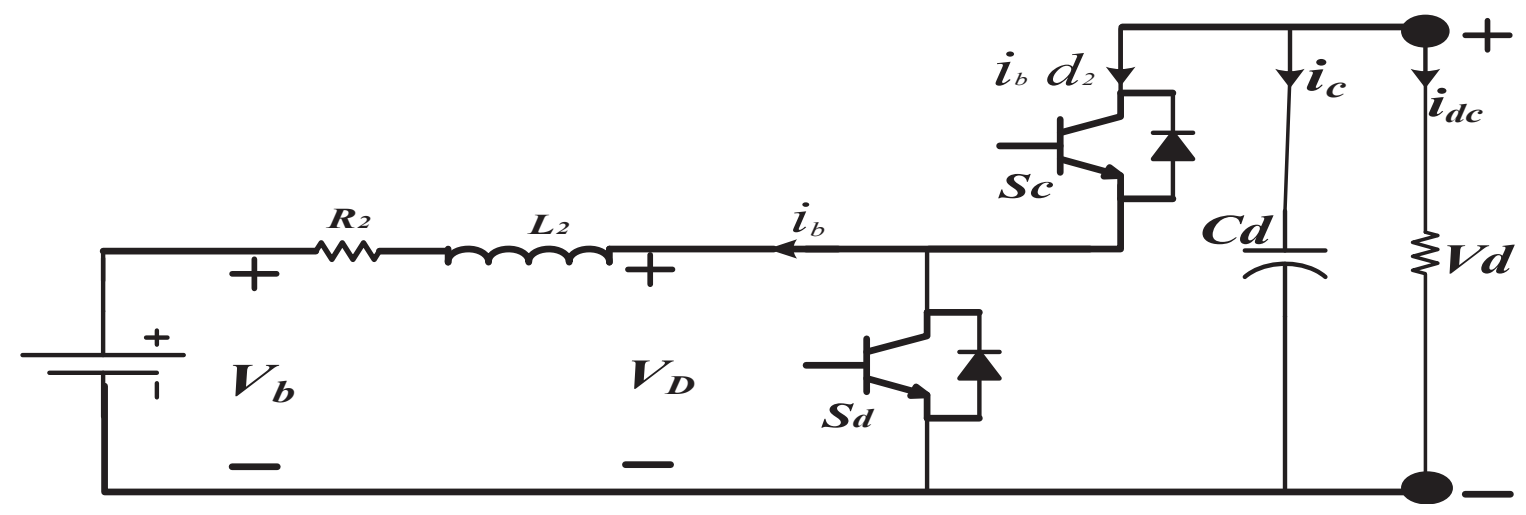

Figure 2.5 The schematic diagram for the battery with the bi-directional DC-DC converter interface to the DC bus

It consists of two switches $\mathrm{Sc}$ and $\mathrm{Sd}$ have different operation purposes and never operate at the same time. When Sc is on, the converter is going to work in buck mode. To move the power from the DC bus to the battery, charging purpose. When Sd is on, the converter is going to work in boost mode, in which the power flows from the battery to the DC bus, for discharging operation. The control algorithm of the battery converter was designed based on the formulas (5), (6) and (7).

$V_{D}-V_{b}=L_{2} \frac{d I_{b}}{d t}+R_{2} I_{b}$

$V_{D}=V_{d} * d_{2}$

$i_{1}\left(1-d_{1}\right)-i_{a c}-i_{d c}-i_{b} * d_{2}=C_{d} \frac{d v_{d}}{d t}$

The control scheme for the bidirectional DC-DC converter is shown in Figure 2.6. The main target of the bidirectional DC-DC converters of the battery banks is to regulate the DC bus voltage. A dual-loop control is used to provide stable DC link voltage. The external voltage-controlled loop is used to generate a reference charging current for the internal 
current controlled loop. The input of the PI controller is the error between the reference and the measured DC bus voltage for the system ${ }^{\left(V_{d}^{*}-V_{d}\right)}$. This error multiplied by -1 and the output is the reference current ${ }^{\left(i_{b}^{*}\right)}$. The internal current control loop will compare the measured current signal with the reference current flow through the converter. The output of the former is set as input for another PI controller for the inner controller.

Based on (5) the battery current ${ }^{\left(i_{b}\right)}$ will be obtained, and the duty cycle ${ }^{d_{2}}$ is estimated by (6) and (7). The injected current $I_{i n}=i_{1}\left(1-d_{1}\right)-i_{a c}-i_{d c}$. For instance, when the DC bus voltage is higher than the reference voltage, the outer voltage controller will generate a negative current reference signal, and the inner current control loop will adjust the duty cycle to force the current flow from the DC bus to the battery, to be charged. During this process, the energy transfers from the DC bus to the battery and the DC bus voltage will decrease the rating amount. If the DC bus voltage is reduced lower than the normal value; the outer voltage control loop will generate a positive current reference signal, which will regulate the current flow from the battery to the DC bus. As a consequence, the extra energy injected from the batteries; the DC bus voltage will increase to the rating value.

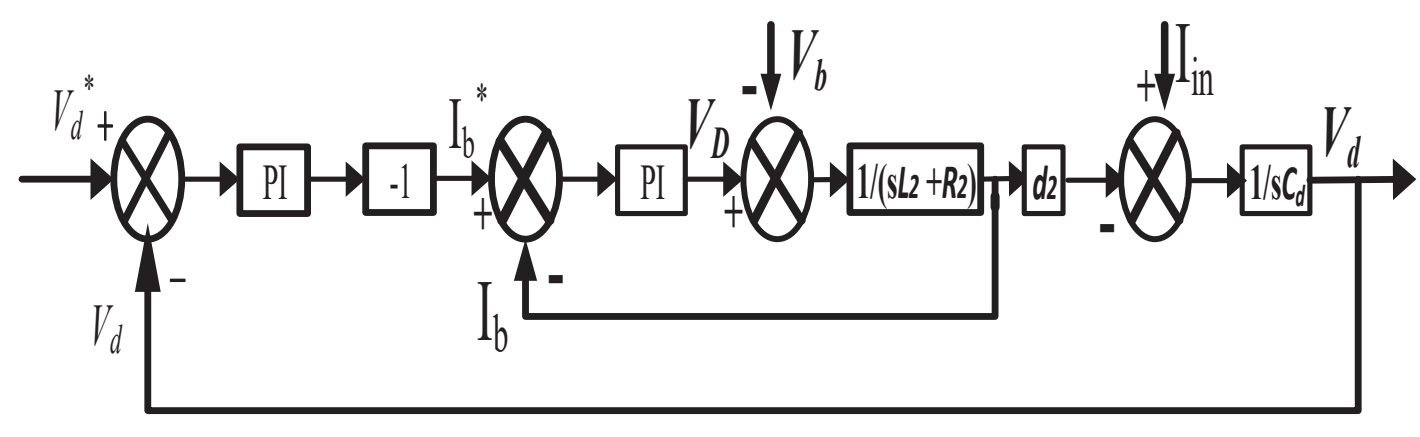

Figure 2.6 The flow chart for the MPPT method (P\&O). 


\subsubsection{Bi-directional DC/AC converter model and control}

In hybrid $\mathrm{AC} / \mathrm{DC}$ microgrid, the frequency and voltage amplitude of the three-phase $\mathrm{AC}$ side is not so robust. Especially, in islanding mode where there is no slack bus connected to the AC side. Usually, the slack generator in the hybrid power system can handle the load with a small varying ratio, but when the pulse load is connected to the AC side, or the total load exceeds the generator's limit, the system frequency may decrease, and the voltage may collapse. Therefore, the main role of the bi-directional DC/AC converter is to regulate the frequency and the voltage magnitude of the AC grid [78]-[81]. Furthermore, in this work, the controller of the DC/AC converter can assure exchange of power between AC and DC grid smoothly. The model of the DC/AC converter can be represented in $\mathrm{ABC}$ coordinate as in (8).

$L_{3} \frac{d}{d t}\left[\begin{array}{c}i a \\ i b \\ i c\end{array}\right]+R_{3}\left[\begin{array}{c}i a \\ i b \\ i c\end{array}\right]=\left[\begin{array}{c}V_{a} \\ V_{b} \\ V_{c}\end{array}\right]-\left[\begin{array}{c}e_{a} \\ e_{b} \\ e_{c}\end{array}\right]+\left[\begin{array}{c}\Delta_{a} \\ \Delta_{b} \\ \Delta_{c}\end{array}\right]$

The above equation can be written in the d-q coordinate as follows:

$L_{3} \frac{d}{d t}\left[\begin{array}{l}i_{d} \\ i_{q}\end{array}\right]=\left[\begin{array}{cc}-R_{3} & w L_{3} \\ -w L_{3} & -R_{3}\end{array}\right]\left[\begin{array}{l}i_{d} \\ i_{q}\end{array}\right]+\left[\begin{array}{c}V_{c d} \\ V_{c q}\end{array}\right]-\left[\begin{array}{c}V_{s d} \\ V_{s q}\end{array}\right]$

The control scheme for the bidirectional AC-DC inverter is shown in Figure 2.7. Two-loop controllers are applied. Active and reactive power control loop to control both frequency and voltage respectively. For the active power loop. Coordination control was developed to control both the frequency of the $\mathrm{AC}$ side and partial control of the DC bus voltage with cooperation with the battery storage. 


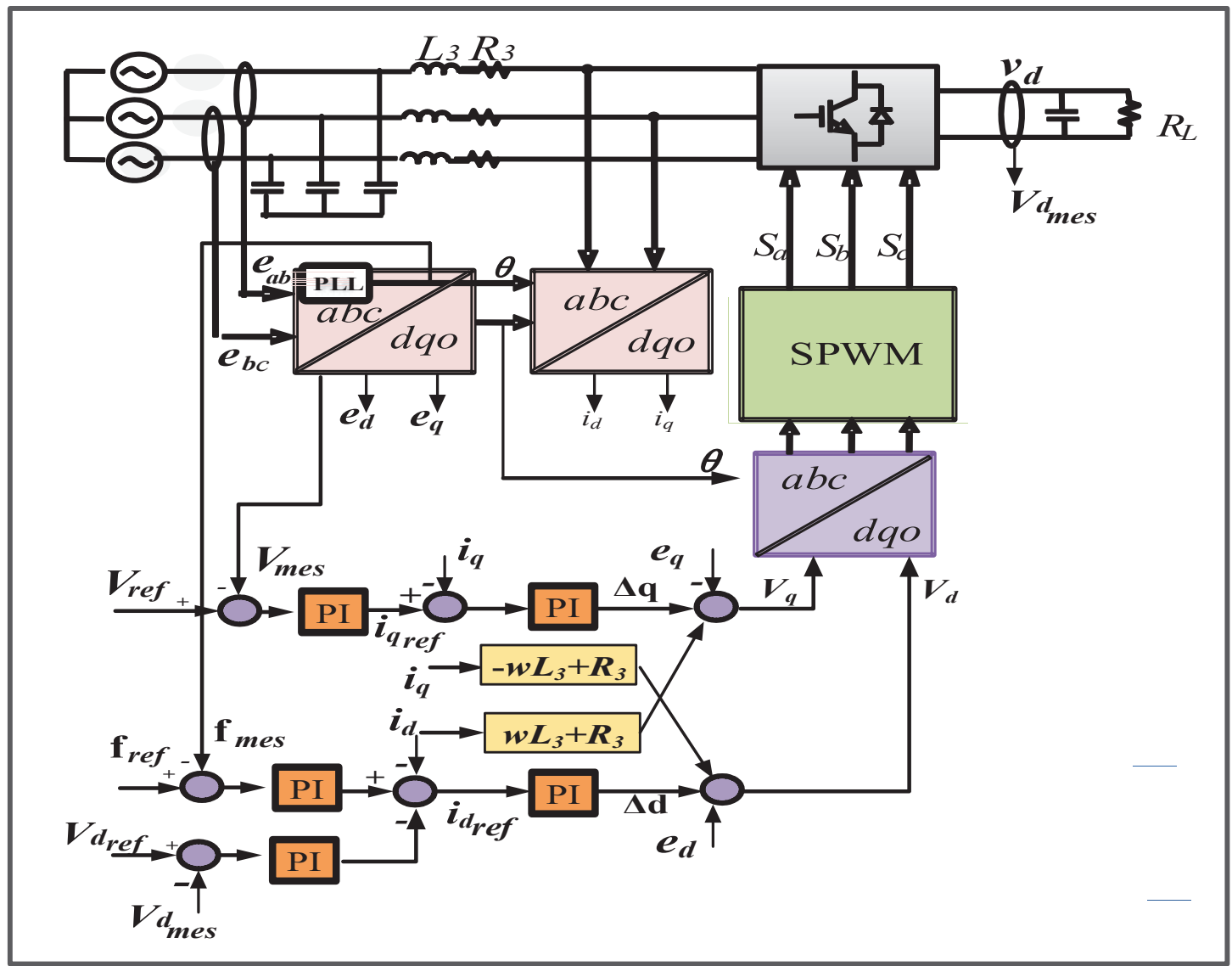

Figure 2.7 Schematic diagram of the AC/DC converter

In the frequency control, the error between the reference frequency and the measured is subtracted from the error between the reference and the measured DC voltage. The output of that summation is set as the input to a PI controller which generates the $I_{d}$ reference. Another control loop to control the reactive power flow helps to control the voltage amplitude. The error between the reference voltage amplitude and the measured voltage amplitude is sent to a PI controller to generate $I_{q}$ reference.

\subsection{CONTROL PARAMETERS DESIGN USING GA.}

GA has turned out to be an excellent optimization tool. It does not require an exact mathematical model of the physical system. Furthermore, speed and robustness are 
prominent properties of this scheme. The proposed design strategy for generating the control parameters is based on GA. It has been used in this paper to optimize the interlinking $\mathrm{DC} / \mathrm{AC}$ control parameters and reduce time consumption compared to the trialerror method. GA is a stochastic search algorithm that emulates biological evolutionary theories to solve optimization problems. It enables parallel search from a population of points. Therefore, it can avoid being trapped in optimal local solution unlike traditional searching methods, which search from a single point. Based on the literature, using GA to optimize the control parameters show success [82], [83]. Such optimal parameters could provide ideal control performance and achieve the desired coordination performance. GA is used to select the optimum combination of the control parameters $\left(K_{p_{-} f}, K_{i_{-}}, K_{p_{-}} v d c, K_{i_{-}} v d c\right.$, $K_{p_{-} v a c,} K_{i_{-}} v a c, K_{p_{-} m}$ and $\left.K_{i_{-} m}\right)$ based on the optimization procedure. GA calls the system dynamic simulation and calculates the integral absolute frequency $(\Delta f)$ and RMS voltage ( $\left.\Delta V_{r m s}\right)$ deviation of the AC Micro-grid, as given in equation (10), which represents the multi-objective fitness function (F).

$F=\operatorname{Min}\left\{\int_{t_{0}}^{t_{f}}|\Delta f| d t+\int_{t_{0}}^{t_{f}}\left|\Delta V_{r m s}\right| d t\right\}$

The objective of GA optimization is to minimize the cost function by accurately choosing the control parameters of the interlinking converter. GA setup for the proposed design optimization procedure is presented in table I. The progression of GA optimization is shown in Figure 2.8-a. The optimum control parameters are indicated in Figure 2.8-b and summarized in Table 2.2. These parameters are introduced to the FL system to provide optimized damping performance. 
Table 2.1 GA setting

Parameter

Number of variables

\begin{tabular}{|c|c|c|}
\hline \multirow[b]{2}{*}{ Bounds } & Variable & {$\left[K_{p f f}, K_{i f f}, K_{p_{-} v d c,} K_{i \_v d c}, K_{p \_v a c}, K_{i \_v a c}, K_{p \_m}, K_{i \_}\right.$} \\
\hline & Lower & {$[0, \quad 0, \quad 0, \quad 0,0,0]$} \\
\hline & Upper & {$[10,5000,10,5000,1,1,5000,1000]$} \\
\hline
\end{tabular}

Populations

Mutation

Crossover

Iterations

Number of running
Value

8
Uniform $80 \%$

Single point

50

3
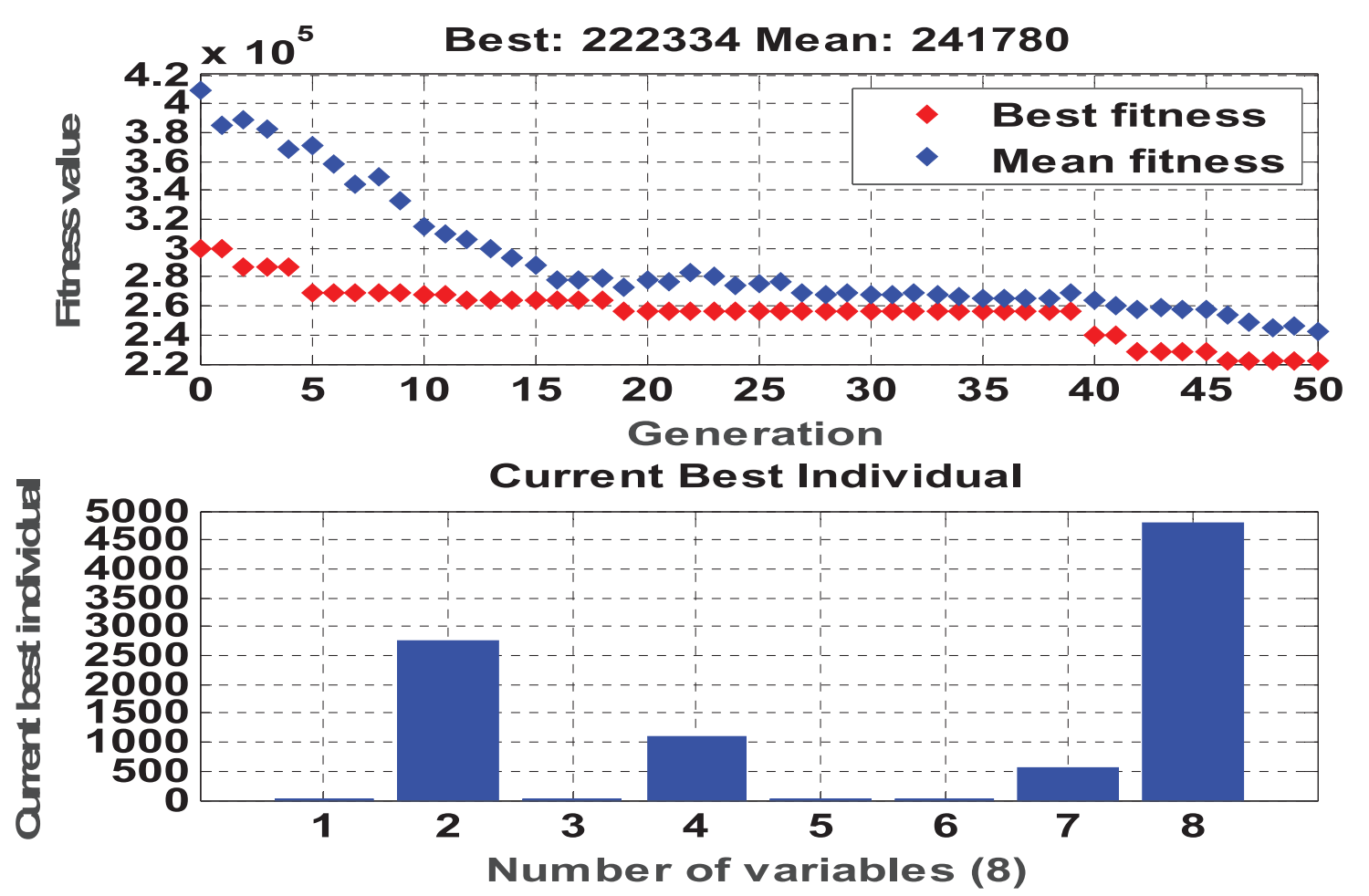

Figure 2.8 GA optimization results (a) Best Fitness, (b) Best Values 


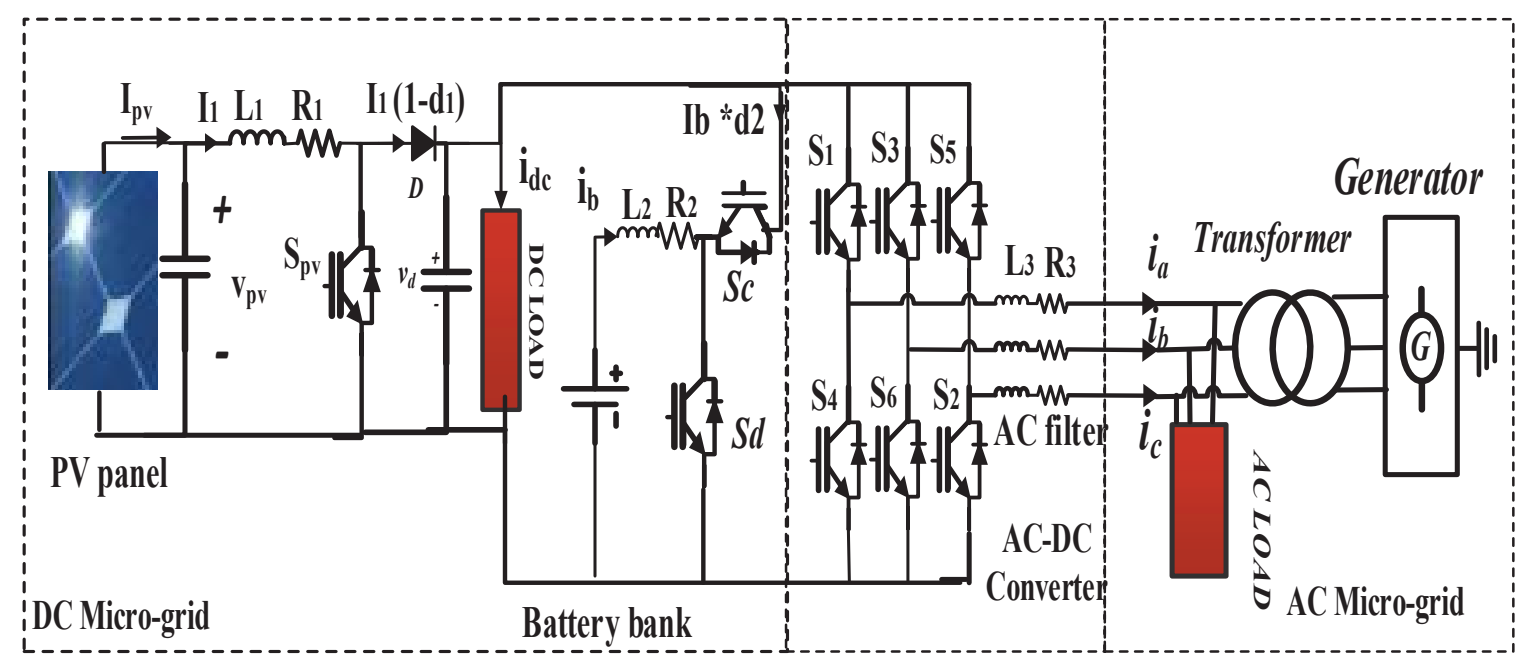

Figure 2.9 The schematic diagram for Matlab/Simulink model of the hybrid AC/DC microgrid Table 2.2 OPTIMIZED FUZZY PARAMETERS

Optimized Variable

$K_{p \_}$

$K_{i f}$,

$K_{p_{-} v d c,}$

$K_{i-} v d c$

$K_{p_{-} \text {vac }}$

$K_{i_{-} v a c}$

$K_{p \_m}$

$K_{i \_m}$
Optimized Value

1.96710133791885

2754.44647432590

0.803382264716479

1110.28219240551

0.0227061401037617

0.0857318115661076

547.070250756871

4804.29071432778 


\subsection{SIMULATION RESULTS}

To investigate the effectiveness of the proposed methodology, a Matlab/Simulink model for a hybrid AC/DC system is built as shown in figure 2.9. The AC microgrid includes a synchronous generator and AC loads. The DC microgrid contains a PV array and Lithium-ion Battery. DC loads are connected to the DC bus as well.

The AC and DC microgrids are interfaced through a VSC as explained earlier. Two pulsed loads are connected to the system, one of them is on the AC side while the other is on the DC side. The simulation time is 4 seconds. The results are depicted in Figures 2.10 through 2.12. It is assumed that the output of the PV array is dropping from $0.8 \mathrm{~s}$ to $1.5 \mathrm{~s}$ due to a dense cloud cover.

This can be seen from Figure 2.10 (a). Consequently, the microgrid control increases the battery output to compensate for this deficit as shown in Figure 2.10 (b). At the same time during the cloud cover, the output of the generator is increased to share this deficit with the battery. The output of the generator is shown in Figure 2.12 (a). This is done in a rapid and coordinated manner such that the supply to the load remains unaffected.

The load profiles are shown in Figure 2.10 (c) and 2.12 (c). It should be noted that in the case of parameter optimization there's more contribution from the generator, this reduces the stresses on the battery. Further, there's less contribution from the DC microgrid to save its assets. 

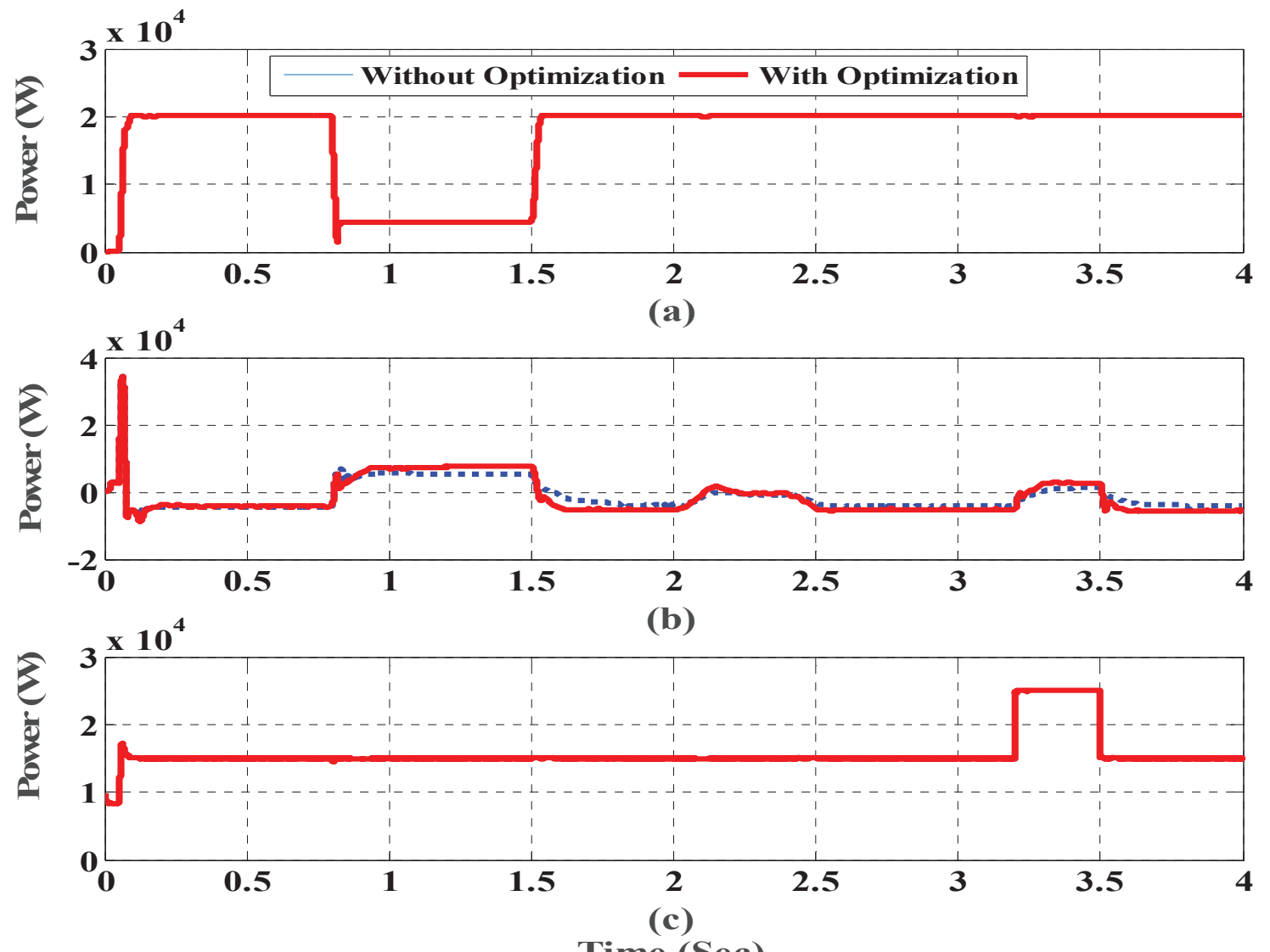

Figure 2.10 Active power flow in the DC microgrid (a) Generated power from the PV array (b) Battery power (c) Load power.

The pulsed load on the DC bus is energized for $0.4 \mathrm{~s}$ (from $2 \mathrm{~s}$ to $2.4 \mathrm{~s}$ ) and the second pulsed load is energized from $3.2 \mathrm{~s}$ to $3.5 \mathrm{~s}$ ). The developed controller acted rapidly and mitigated the pulsed load by sharing the power with the appropriate ratio to avoid any disturbance. In the case when the DC pulsed load is energized, the power flow in the inverter is reversed. This is shown in Fig. 2.12 (b) where the power is negative. This indicates the reversal of the power flow direction to be from the $\mathrm{AC}$ side to the $\mathrm{DC}$ side to share the pulsed load and compensate the DC bus voltage. The stable operation of the microgrids is indicated by variation of the voltage and frequency. 
It can be seen from Figure 2.11, with considering the variations above and dynamic operation scenarios. The voltage and frequency variations are within the allowed limits by the standards.

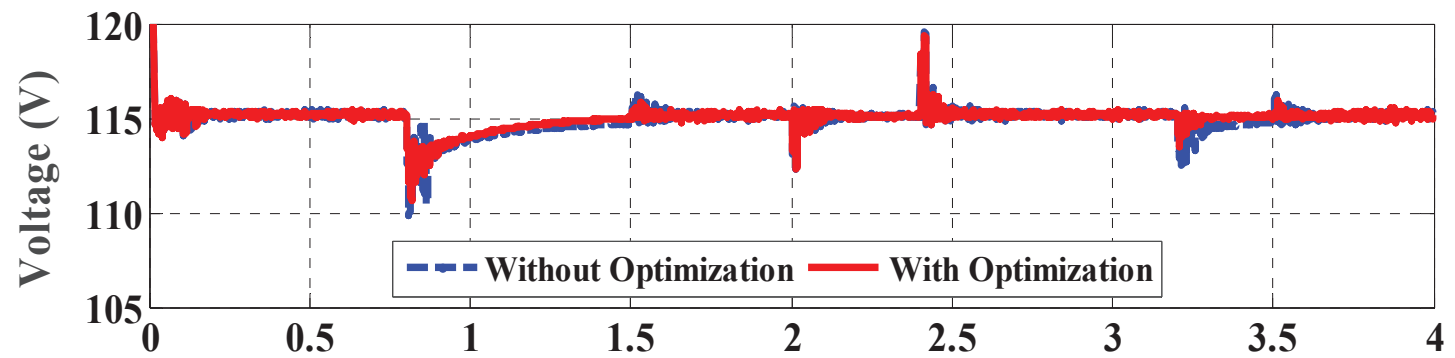

(a)

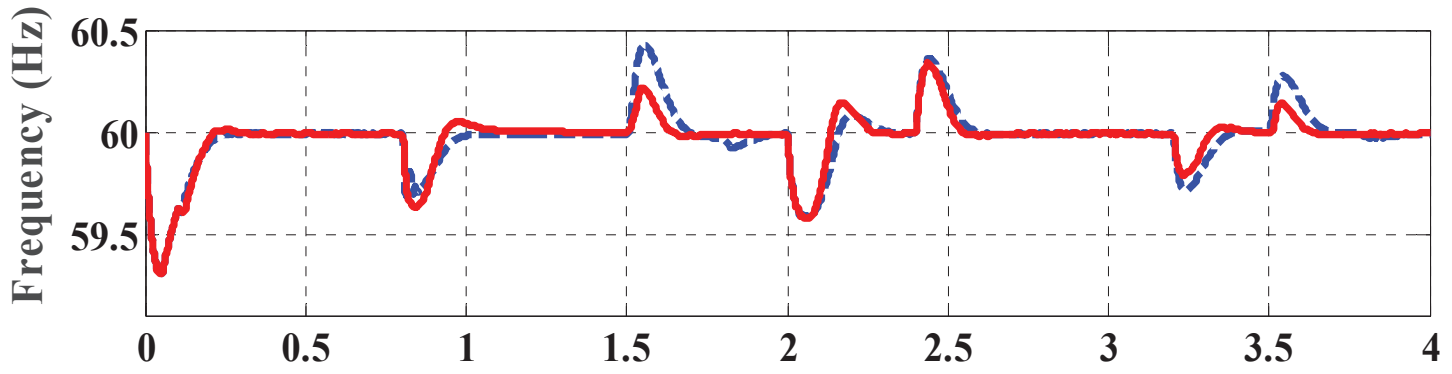

(b)

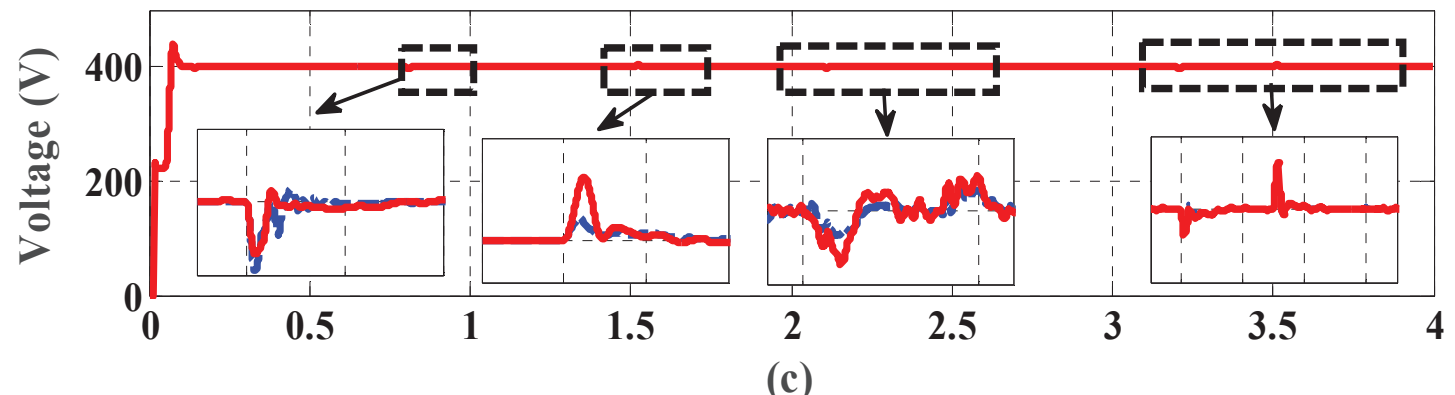

Time (Sec)

Figure 2.11 (a) AC bus voltage (RMS value) for phase a (b) AC microgrid frequency (c) DC bus voltage. 

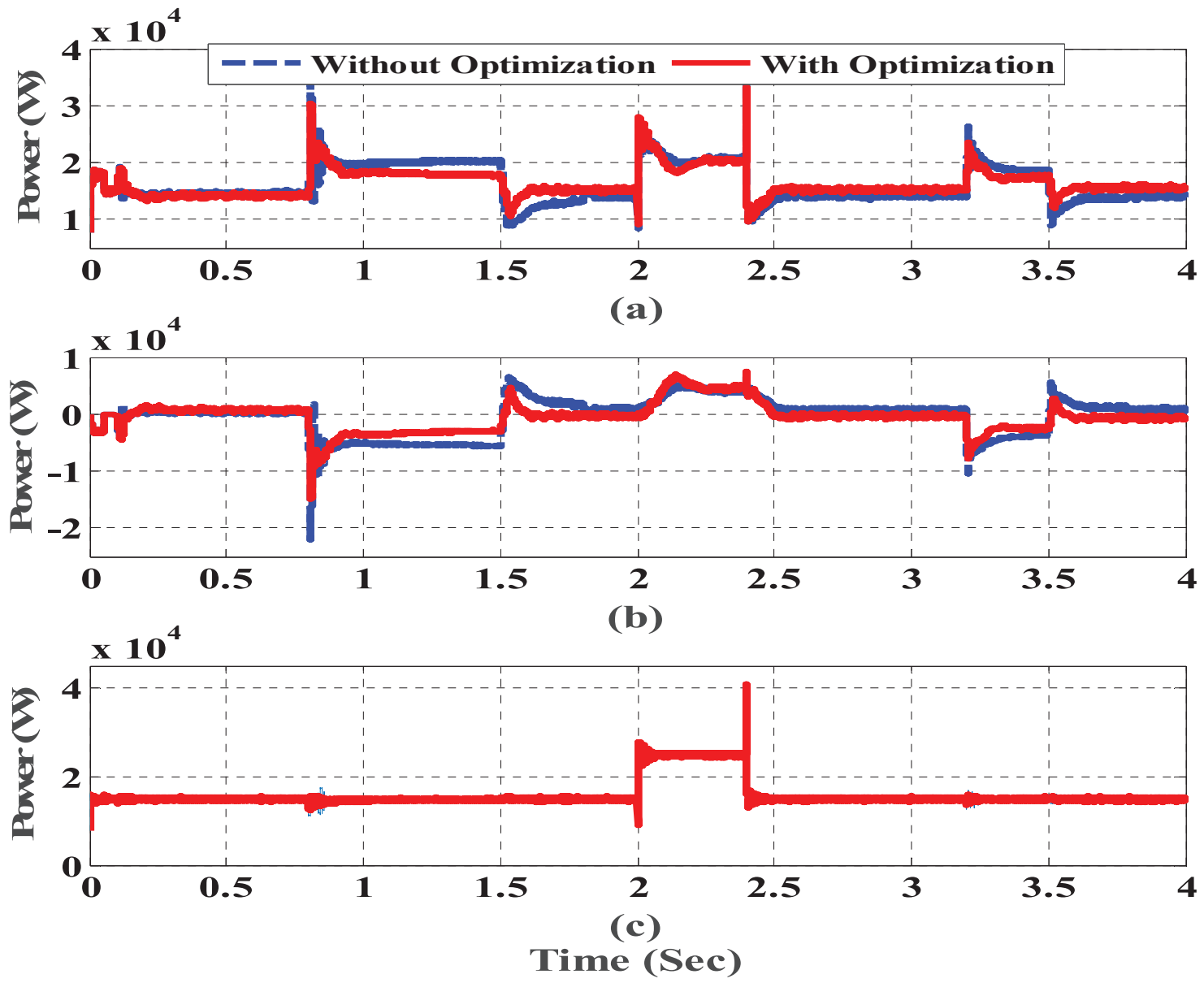

Figure 2.12 Active power flow in the AC microgrid (a) Synchronous generator output power (b) Inverter power at the PCC(c) Load power

Although the voltage variations are high as shown in Figure 2.11 (a), however, these variations are still within the allowed limits. The DC bus voltage is shown in Figure 2.12 (c) and it is stable around the reference value $(400 \mathrm{~V})$. Due to the variations in the generator loading, the frequency is fluctuating. It can be seen that with the optimized parameter scenario using GA, the fluctuations are less. Whereas in the non-optimized case the frequency fluctuations are exceeding the limits in some instances. This may cause triggering the under/over frequency functions on the protection relays. These fluctuations 
are limited through the optimized parameter approach which indicates the effectiveness of the proposed method in this paper.

\subsection{CONCLUSION}

In this work, a genetic algorithm-based hybrid $\mathrm{AC} / \mathrm{DC}$ microgrid control is proposed to ensure system stability. The coordination control scheme is proposed for the IC converter to maintain stable system operation under various load and resource conditions. The Matlab/Simulink simulation verify the tuned coordinated control system. Different resource conditions and load capacities are tested to validate the control method. The simulation results show that the hybrid grid can operate steadily in islanding mode. Stable AC and DC bus voltage can be guaranteed when the operating conditions or load capacities change. The power is smoothly transferred when load condition varies. 


\section{Chapter 3 Non-Intrusive Load Monitoring (NILM) Techniques for Load Forecasting}

The new vision for moving the power system to a smart grid enables a variety of smart applications at different power system infrastructure's levels. Households represent a large part of the grid infrastructure which could not be considered as a smart grid without smart households integrated into it. Short Term Load Forecasting (STLF) is an essential tool needed in the management and control techniques required for households to be smart. The STLF at this level of the grid is very challenging due to the high percentage of uncertainty in the load demand, influenced by customer behavior, which is too stochastic to predict. In this dissertation, a new approach for STLF of household load demand is employed based on artificial neural network (ANN) and a pre-processing stage of a Non-Intrusive Load Monitoring (NILM) techniques. The NILM techniques extract the individual load pattern from the available historical aggregated load demand. These new features increase the training data window for the ANN forecaster and achieve a significant enhancement for its prediction performance. By comparing the new approach with the state of the art techniques in household load forecasting, the proposed method outperforms feed-forward artificial neural network (FFANN) regarding RMSE. Two techniques of NILM were used to emphasize the correlation between the NILM disaggregation accuracy performance and the load forecasting enhancement performance. 


\subsection{Introduction}

The contemporary power system is moving forward towards a smart grid, whereas several innovations at different power system infrastructure's levels occur. Furthermore, the unprecedented penetration increase of renewable energy, time-varying load demand, and Electric Vehicle (EV) in the distribution grid, add an extra burden on the grid regarding the complexity and uncertainty. Therefore, the stability of the grid is experiencing extraordinary challenges due to the intermittency of renewable generations, the complexity of utility-customer interaction, and dynamic behaviors. A considerable section of this complex distribution network represented on Households which are responsible for a significant portion of electrical energy consumption. Enabling the active participation of households' demand-side response (DSR) instead of considering it as a passive load will have a positive impact on the power system stability.

In this case, forecasting household load consumption is essential for residential DSR program. Accurate short-term load forecasting (STLF) on the household level can significantly facilitate the power system operations. STLF refers to the expectation of the power demand in the next hour, next day, or up to a week. STLF is the essential tool needed in the management and control techniques required for households to be smart. STLF at this level of the grid is very challenging due to the high percentage of uncertainty and volatility in the load demand, influenced by customer behavior, which is too stochastic to predict.

Based on the state of the arts in the field of load forecasting, the prediction methods have been used include time-series analysis such as exponential smoothing and 
autoregressive regressive integrated moving average (ARIMA) and machine learning approaches such as artificial neural networks (ANN) and support vector machine (SVM). In [84] adopted autoregressive integrated moving average model for a day ahead load forecasting was presented. In which the prediction mechanism is based on grouping the targeted day with the similar meteorological days in the historical data. In [85] Radial basis function (RBF) neural network had been used to address the STLF. In [86] a combination between RBF neural network and the adaptive neural fuzzy inference system (ANFIS) was presented to adjust the prediction by taking into consideration the real-time electricity price. In [87] it is proposed a neural network based predictor for very short-term load forecasting.

It takes only the load values of the current and previous time steps as the Input to predict the load value at the subsequent time step. In [88] an ensemble of extreme learning machines (ELMs) to learn and forecast the total load of the Australian national energy market was used. In [89] a dedicated input selection scheme to work with the hybrid forecasting framework using wavelet transformation and the Bayesian neural network was presented. Based on the previous literature, the techniques used for load forecasting can be concluded in three categories. The first is avoid the uncertainty by Clustering/classification techniques which group similar customers, days or weather in the hope of reducing the variance of uncertainty within each cluster [90]. However, the performance is heavily dependent on the data. The second is trying to cancel out the uncertainty by using aggregated smart metering data so that the aggregated load exhibits mostly regular patterns and more easier to predict, yet the prediction is visible only at an aggregated level. The 
third is trying to separate the regular pattern from the other component of load profile such as uncertainty and noise by pre-processing techniques, mostly spectral analysis such as empirical mode decomposition (EMD) [91], Fourier transforms [92], and wavelet analysis [93]. This method can be ruled out in household load forecasting due to the high uncertainty proportion of the load pattern.

All these previous work for load forecasting techniques are suitable for the aggregated level such as system or community level. There is a little work on the literature regarding load forecasting at the household level. For example, the first two trails to address load forecasting at household level in [94], [95]. In which, a functional time series forecasting approach was proposed. However, it used daily median absolute errors (DMAE) which is not the commonly used metric. In that way, it is improper to assist as a benchmark for experimental assessments. The standard metric used to assess the forecaster performance is mean absolute percentage error (MAPE). Recently, some work is done in the area of load forecasting using the deep artificial neural network (DANN). In [94], the accuracy of load forecasting for industrial customers is improved by using DANN. It was the first attempt to use a factored conditional restricted Boltzmann machine, for household load forecasting. However, it outperforms regarding the performance than artificial neural network and support vector machine. In [96], another deep neural network (DNN) approach called long short-term memory (LSTM) is used. Although deep learning has received high expectation in forecasting community, the state of the arts indicates that deep learning is more prone to over-fitting compared with artificial neural networks [97]. The problem is expected due to the existence of more parameters and relatively fewer data. For that reason, another work 
based on a pooling-based deep recurrent neural network (PDRNN) is proposed in [98] to tackle the overfitting issue. However, the methodology was tried to avoid the over-fitting problem by increasing the data dimension which is the historical data of the neighbors to the neural networks. The main drawback here that this method is pooling the data of the neighboring household to increase the data set. Which most probably not available in another place for privacy policy.

In this work, a new approach for STLF of household load demand is employed based on artificial neural network (ANN) and a pre-processing stage of a Non-Intrusive Load Monitoring (NILM) techniques. The NILM techniques extract the individual load pattern from the available historical aggregated load demand. These new features increase the training data window for the ANN forecaster and achieve a significant enhancement for its prediction performance. By comparing the new approach with the state of the art techniques in household load forecasting, the proposed method outperforms ARIMA, SVR, and ANN regarding root mean square error RMSE. We use two different disaggregation algorithms called Factorial Hidden Markov Model (FHMM) and Combinatorial Optimization (CO) to extract the power demand profile for individual appliances from the main aggregated Household smart meter. Two techniques of NILM were used to emphasize the correlation between the NILM disaggregation accuracy performance and the load forecasting enhancement performance. Figure 3.1 shows the block diagram of the whole proposed system. 


\subsection{Non-Intrusive load monitoring}

Non-intrusive load monitoring or energy disaggregation is a computational approach for estimating the individual appliances power consumption from a single meter which measures the aggregated power consumption of multiple appliances. This research started with the vital work of George Hart [99], [100] in the mid-1980s. His earliest work described a signature taxonomy of feature. However, his focus was on extracting only transitions between steady-states. Following Hart's lead, many NILM algorithms designed for lowfrequency data (1 Hz or slower). But only extract a small number of features. Regarding the high frequency (sampling at $\mathrm{kHz}$ or even $\mathrm{MHz}$ ), there are several examples of manually engineering feature extractors discussed in the literature [101], [102]. Humans can learn to detect appliances in aggregate data by eye, especially appliances with feature-rich signatures. Hand-engineering feature extractors could be considered for analyzing profiles with rich features. But this would be time-consuming, and the resulting feature detectors may not be robust to noise and artifacts. Hand-engineer feature extractors such as scaleinvariant feature transform [103] (SIFT) and difference of Gaussians (DoG) was the dominant approach to extract features for image classification prior 2012. But, in 2012, during the competition of ImageNet Large Scale Visual Recognition, different algorithms achieved an excellent performance and did not use hand-engineered feature detectors. Instead, they used different disaggregation algorithm which automatically learned to extract a hierarchy of features from the raw image. Using the same approach in this paper, we will use various disaggregation algorithms called Factorial Hidden Markov Model (FHMM) and Combinatorial Optimization (CO) to extract the power demand profile for 
individual appliances from the main aggregated Household smart meter. The detailed illustration of these algorithms introduced later in this section.

\subsubsection{Dataset and Non-Intrusive Load Monitoring Tool Kit (NILMTK).}

There are numerous problems in energy sector need to be tackled. A lot of them need prediction tasks and data analysis, areas where techniques such machine learning and data mining can prove invaluable. As is the case in this paper, to use the NILM to enhance the household forecasting performance, the energy consumption of each household has to be available. For this reason, the data from the Reference Energy Disaggregation Data Set (REDD) released in 2011[104] is used.

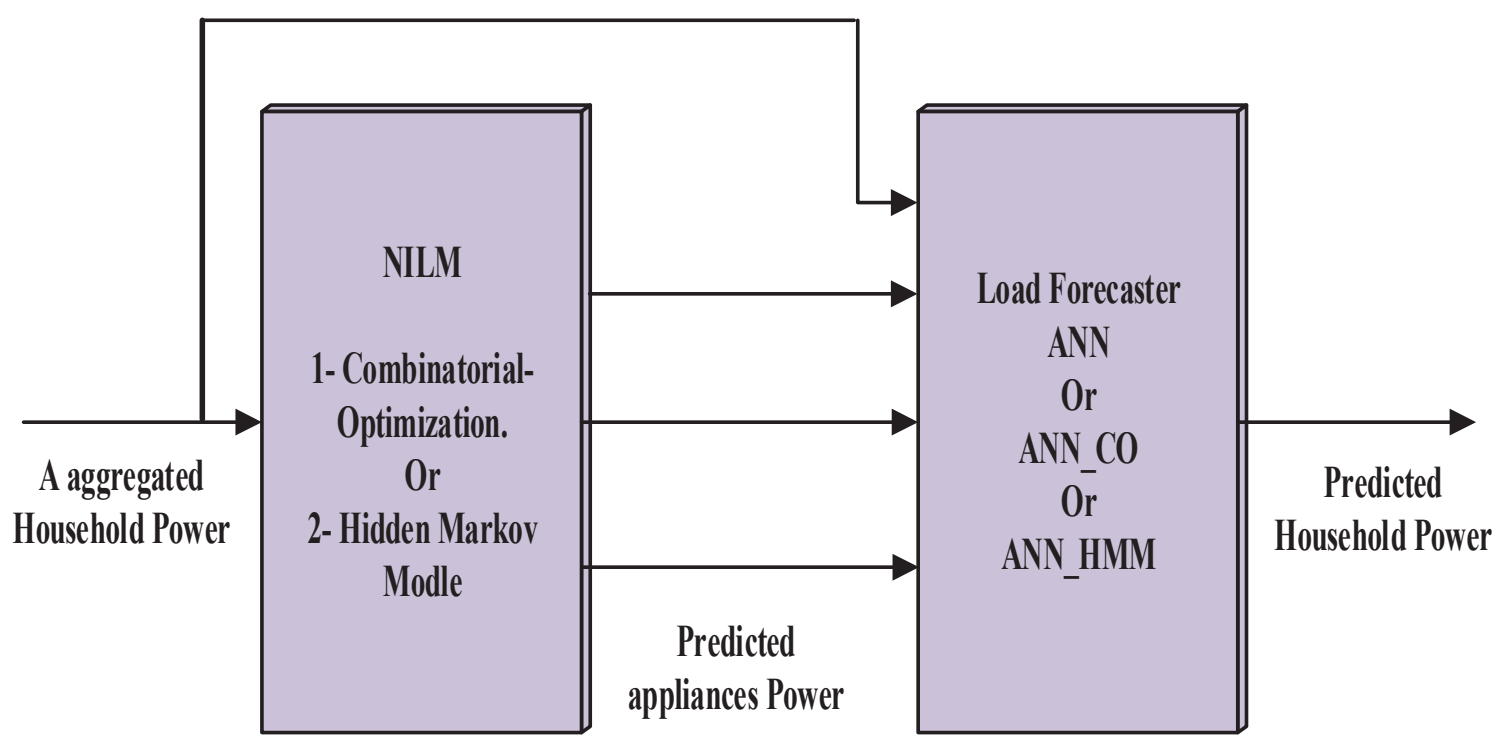

Figure 3.1 Block diagram for the proposed household load forecasting approach.

The first publicly available dataset collected mainly to assist the research of NILM. The dataset comprises of whole-home and device/circuit specific electricity consumption for some real houses over several months' time. For each house, the whole home electricity signal is recorded. The measurement placed as follows: a voltage monitor on one phase 
and current monitors on both phases of power. All these recorded at a high frequency $(15 \mathrm{kHz})$. Furthermore, there are 24 individual circuits in the home, which labeled with its category of appliances, recorded at $0.5 \mathrm{~Hz}$. Also, there are 20 plug-monitors in the home, recorded at $1 \mathrm{~Hz}$, with a focus on plugged electronics devices where multiple devices are grouped into a single recorded point circuit. It is considered the most popular dataset for evaluating energy disaggregation algorithms. The implementation of the two disaggregation algorithms as mentioned above is done based on the NILMTK [105] to implement the disaggregation process. This NILMTK implemented based on Python which provides a massive set of libraries supporting both machine learning and NILM algorithms. Furthermore, Python permits easy implementation in diverse environments including academic settings and is increasingly being used for data science. Figure 3.2. presents the NILMTK's process block diagram from the import of the datasets to the estimation of different disaggregation algorithms over multiple metrics [105].

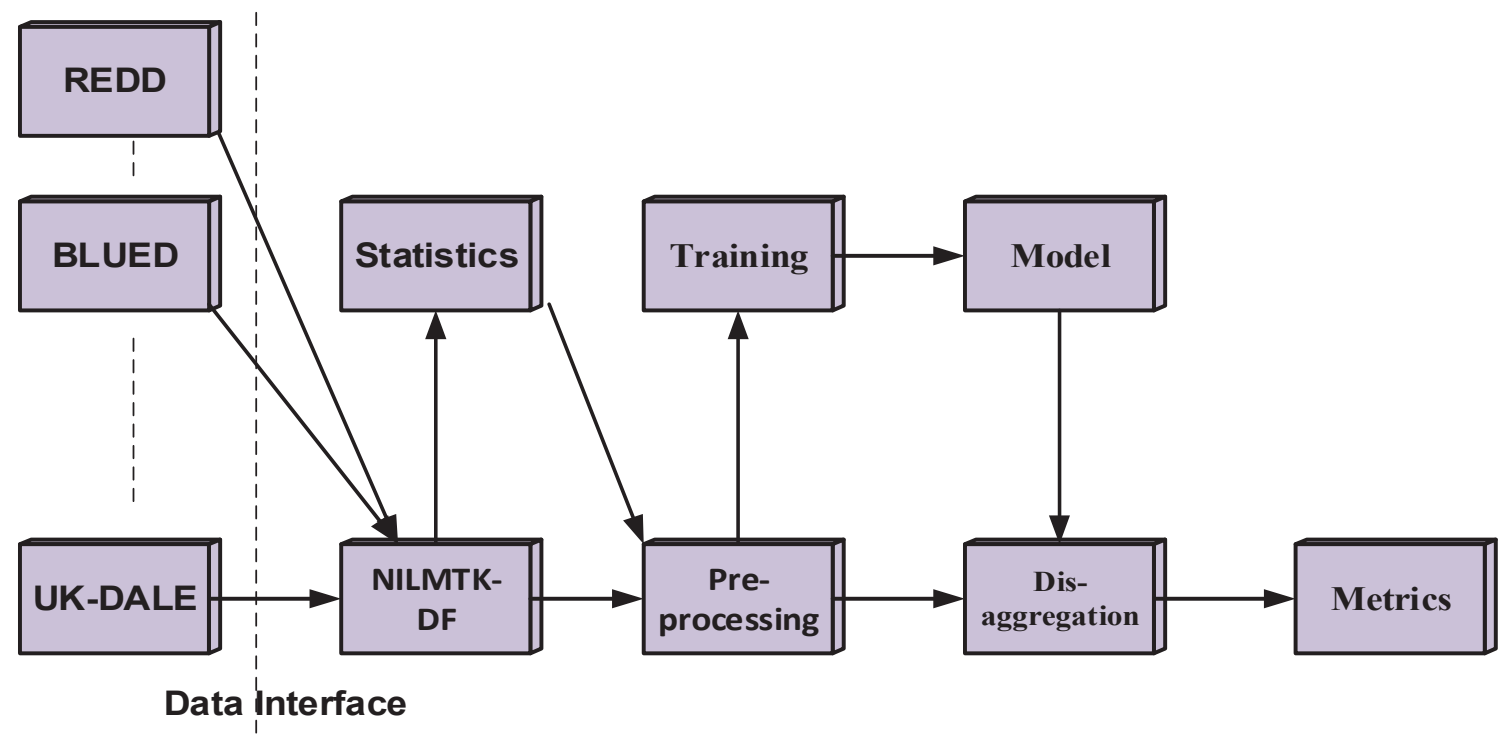

Figure 3.2 NILMTK's process block diagram 


\subsection{2 . Combinatorial Optimization (Optimization Methods)}

Optimization approaches require the existence of appliance signature libraries with all possible combinations of power demands of the appliances it is needed to disaggregate. If we include the combinations of all the installed appliances in a household, then this optimization approach is called brute-force. However, as was stated in [106] due to memory limitations, brute-force methods are impossible to be applied in an embedded system. Thus the load identification requires the definition of an objective function and its minimization. Considering the aggregate data $\bar{x}$ and an appliance set $=$ $\left[x_{1}, \ldots \ldots \ldots \ldots, x_{N}\right]$, the problem is formulated as $[106]$ :

$\min _{1 \leq n \leq N}\left\|\bar{x}-\sum_{n=1}^{N} x_{n}\right\|$

The most critical algorithm in this domain is the Combinatorial Optimization which minimizes the difference between the sum of the measure aggregate power and predicted appliance power [107]. This method was also used by Hart in [108]. The computational complexity is $o\left(K^{N} T\right)$, where $\mathrm{K}$ is the number of appliance states, $\mathrm{N}$ the number of appliances and $\mathrm{T}$ the number of times slices used in the implementation. The problem complexity increases as the number of different loads in the aggregated signal increases, since the algorithm should take into account all possible combinations of appliances contained into the training set. The problems with this approach are [107]:

1. The presence of new loads in the aggregated signal.

2. The sequential dependencies among the appliances are neglected.

3. Appliances with similar consumption are difficult to distinguish. 
Thus optimization methods address mainly disaggregation for the most power-hungry devices and a limited number. In [109] it is discussed the two commonly cited disadvantages of this approach which are the decreasing of accuracy with the number of appliances and level of noise.

Factorial Hidden Markov Model belongs to the category of Temporal Graphical Models which is a class of probabilistic models. Such models have been applied previously to many real-world problems like speech recognition. The most straightforward representation of sequence data is through the use of a Markov chain which is a sequence of discrete variables. State transitions of devices are handled by the hidden Markov model (HMMs) which is a statistical tool. Each variable is described by its real power consumption in addition to other useful information such as duration of the on and off periods and time of use during the day/week. Thereby, at an instant of time $t$ of a period T, $\mathrm{t} \in \mathrm{T}$, the aggregate consumption is $\bar{x}(t)$ and needs to be broken down to number of appliances $z_{t}^{n}$, where $\mathrm{t} \in \mathrm{T}$ and $\mathrm{n} \in \mathrm{N}$ with $\mathrm{N}$ the number of appliances. The value of each device $z_{t}^{n}$ at any time corresponds to one of the $\mathrm{K}$ states of the trained model of the appliances [106]. The mathematical representation of the a HMM represented through Eq 2. The behavior of a HMM can be completely defined and inferred by three parameters. First the probability of each state of the hidden variable at the time $t$ can be represented by the vector $\pi$ such that

$\pi_{\mathrm{K}}=\rho\left(\mathrm{z}_{\mathrm{t}}=\mathrm{k}\right)$

Second, the transition probabilities from state $i$ at $t$ to state $j$ at $t+1$ can be represented by the matrix A such that, 
$A_{i, j}=\rho\left(z_{t+1}=j \mid z_{t}=i\right)$

Third, the emission probabilities for $\mathrm{x}$ are described by a statistical function with parameter $\emptyset$ which is commonly assumed to be Gaussian distributed such that,

$x_{t} \mid z_{t}, \emptyset \sim \mathcal{N}\left(\mu_{z_{t}}, \tau_{z_{t}}\right)$

Where $\varnothing=\{\mu, \tau\}$, and $\mu_{z_{t}}, \tau_{z_{t}}$ are the mean and precision of a state's Gaussian distribution. Finally, Equations 2, 3, 4 can be used to compute the joint likelihood of a HMM:

$\rho(\mathrm{x}, \mathrm{z} \mid \theta)=\rho\left(\mathrm{z}_{\mathrm{t}} \mid \pi\right) \prod_{\mathrm{t}=2}^{\mathrm{T}} \rho\left(\mathrm{z}_{\mathrm{t}+1} \mid \mathrm{z}_{\mathrm{t}}, A\right) \prod_{\mathrm{t}=1}^{\mathrm{T}} \rho\left(\mathrm{x}_{\mathrm{t}} \mid \mathrm{z}_{\mathrm{t}}, \emptyset\right)$

Where the set of all model parameters which must be found for each appliance during the training phase is represented by $\theta=\pi, A, \emptyset$.

Therefore, when applying an HMM for Energy Disaggregation, it is needed to tune the $\theta$ parameters for each appliance during the training phase and afterwards, given a sequence of power signal $\bar{x}$ to find the optimal sequence of discrete states z. Their ability to handle daily operation consumption and the information about state transition of devices makes them a suitable solution for the problem. The complexity of the

disaggregation using HMMs is $O\left(K^{2} T\right)$, where $\mathrm{K}$ is the number of states of all the appliances and $\mathrm{T}$ is the number of the time slices, i.e., who many times the algorithm is required to be applied [106]. As it is shown the complexity is exponential with regard to the number of appliances while re-training is needed when a new group of appliances is added [110]. In [111], the HMMs were used for appliance load recognition, and it was also 
shown that they are useful in the field of NILM. Finally, Oliver Parson in [109] is using HMMs to disaggregate an energy signal using generalized appliance model, and as a result, it was possible to extract consumption of individual devices without any manual labeling. But he used low-frequency Smart Meter data because of lack of high-frequency data and smart metering infrastructure supporting such high rates. Although the HMM is a powerful technique, the method for the inference of hidden states is often affected by local minima [112]. To overcome this limitation, variants of HMMs are used like the Factorial HMM (FHMM). The concept is that the output is an additive function of all the hidden states. In the model, each observation is dependent upon multiple unknown variables [113]. The graphical model is given in Figure 3.3. Similarly, the joint likelihood of an FHMM as stated in [114] is computed by,

$\rho\left(x^{(1: N)}, z \mid \theta\right)=\prod_{n=1}^{N} \rho\left(z_{t}^{(n)} \mid \pi\right) \prod_{t=2}^{T} \prod_{n=1}^{N} \rho\left(z_{t+1}^{(n)} \mid z_{t}^{(n)}, A\right) \prod_{t=1}^{T} \rho\left(x_{t} \mid z_{t}^{(1: N)}, \emptyset\right)$

Where 1: $\mathrm{N}$ symbolizes a sequence of appliances $1, \ldots, \mathrm{N}$. However, the computational complexity of both learning and disaggregating is greater for FHMMs compared to HMMs. This is due to the conditional dependence of the Markov chains.

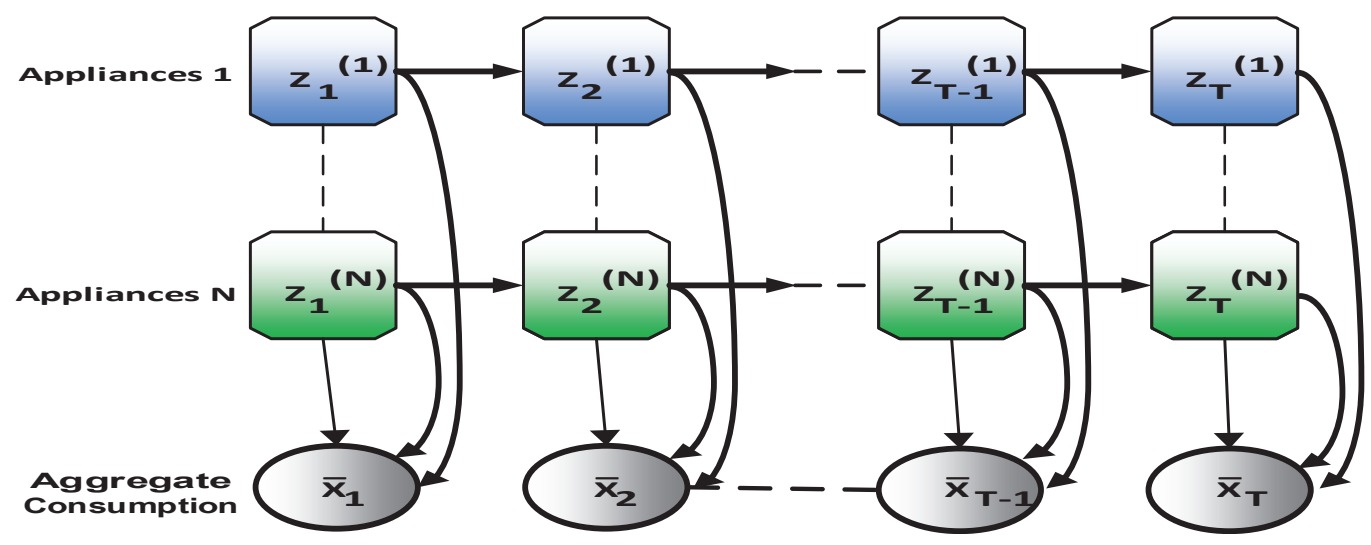

Figure 3.3 Illustration of a Factorial Hidden Markov Model. 


\subsubsection{Load disaggregation}

In this part, the performance of the NILM algorithms is presented. For preparing the appliances power profiles for load forecasting stage, the data are reformed to be hourly power demand as in Figure 3.4. And Figure 3.5. Where Figure 3.4. Represent the aggregated data and the disaggregated data by using FHMM algorithm. However, Figure 3.5. Represent the aggregated data and the disaggregated data by using $\mathrm{CO}$ algorithm.
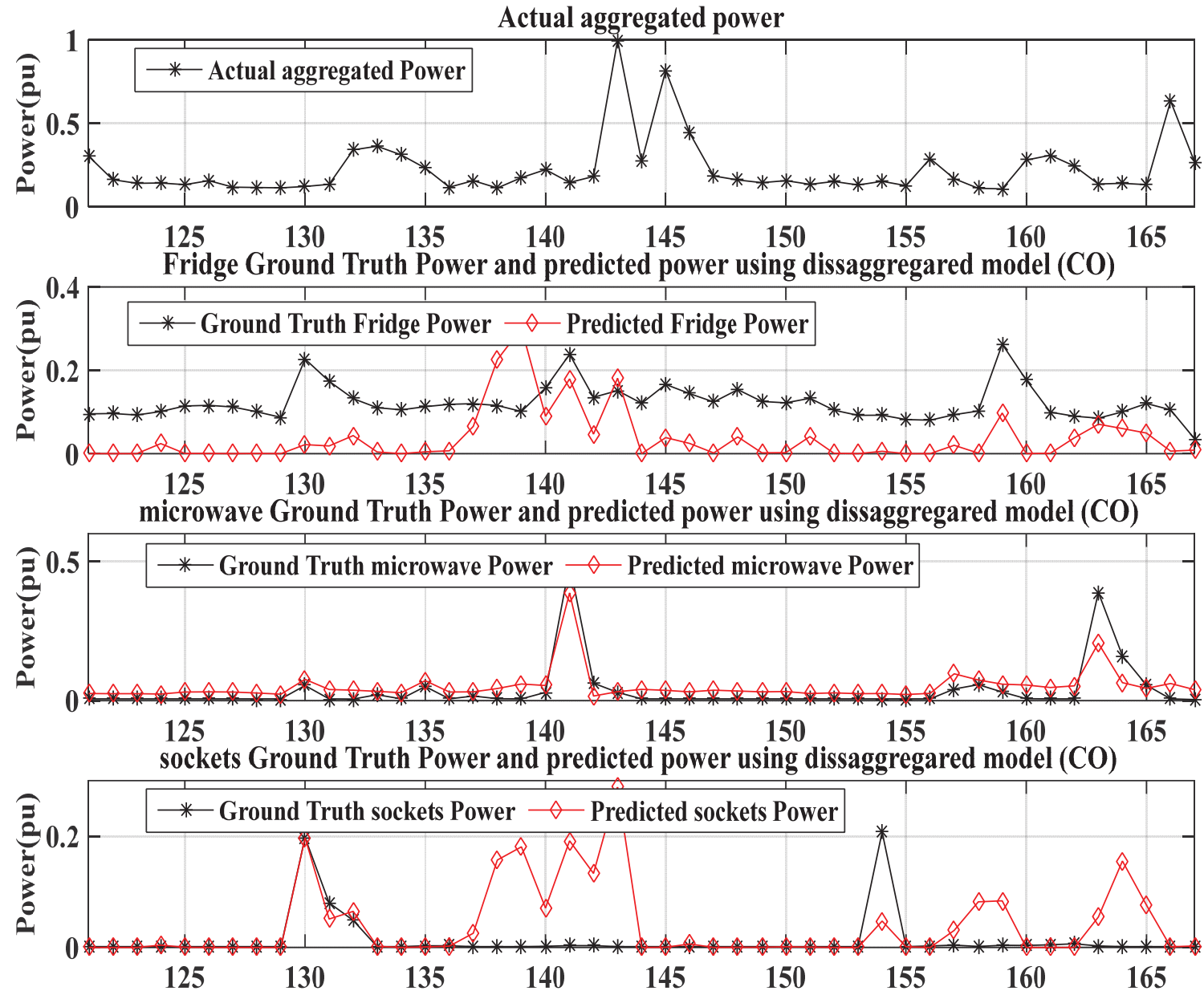

Figure 3.4 Aggregated power for Home (b). Comparison between predicted and ground truth fridge power. (c) Comparison between predicted and ground truth microwave power. (b) Comparison between predicted and ground truth Kitchen Sockets power by using FHMM algorithm for disaggregation. 

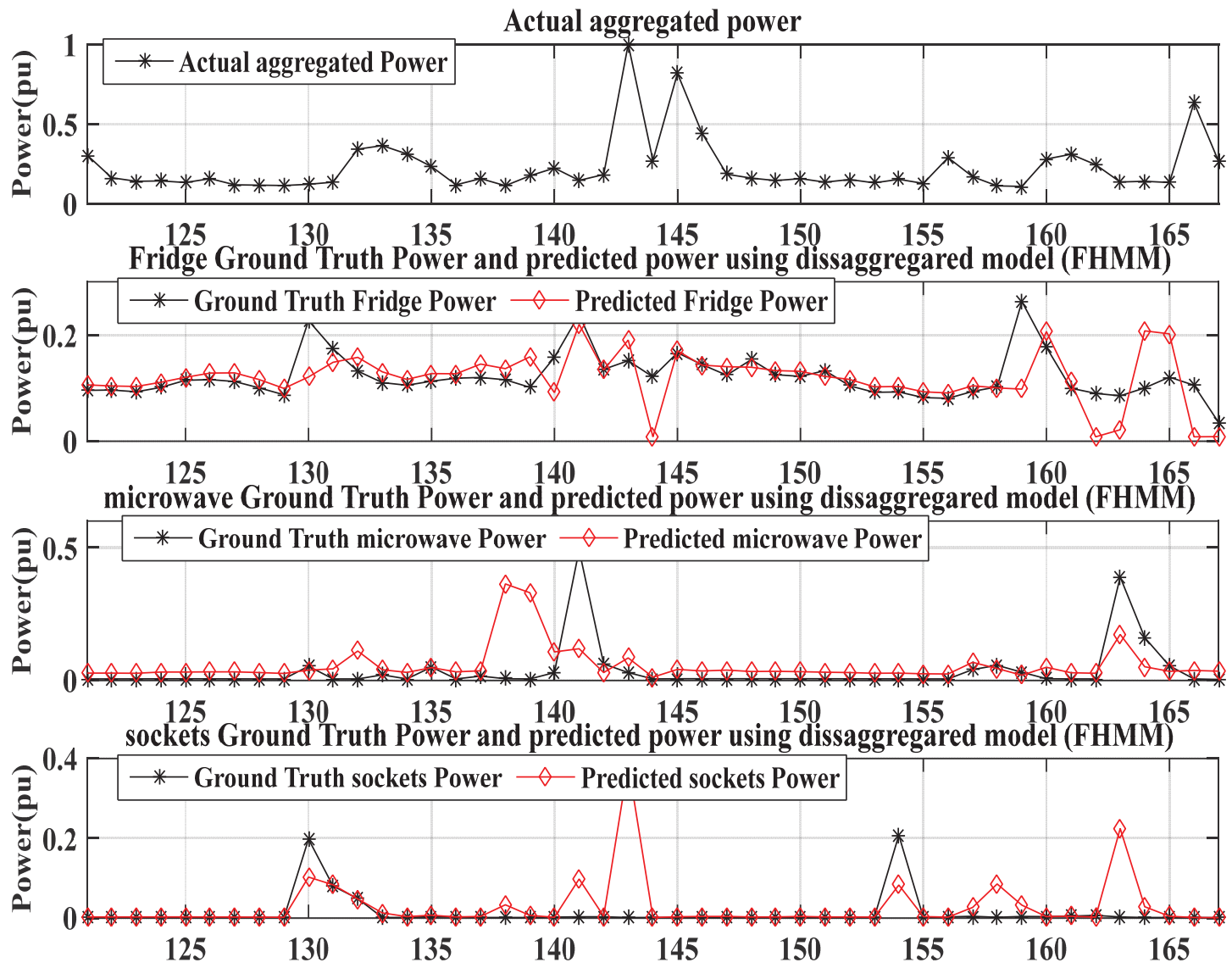

Figure 3.5 (a). Aggregated power for Home (b). Comparison between predicted and ground truth fridge power. (c) Comparison between predicted and ground truth microwave power. (b) Comparison between predicted and ground truth Kitchen Sockets power by using CO algorithm for disaggregation.

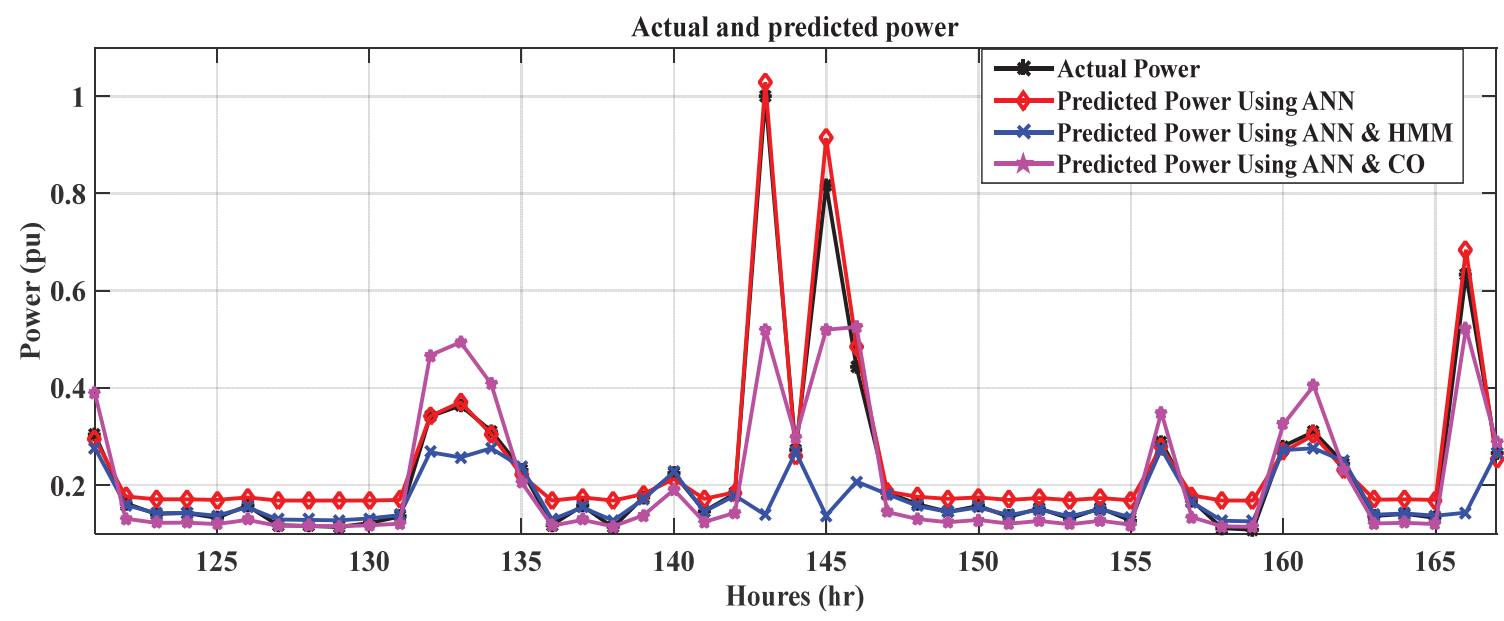

Figure 3.6 Comparison between the actual and predicted power demand with three different algorithms FFANN, FFANN combined with FHMM, and FFANN 


\subsection{The implemented Short-Term Load Forecasting}

A feed-forward Neural Network of the same design as used in [115] is employed. Which is with nine input nodes and one output nodes. One input for the current hour power demand, one for an hour before, one for two hours before, one for a day before, one for the 23 hours before, one for 22 hours before, and three inputs for the three predicted appliances from the current power profile. The output node for the expected hour ahead.

The loads used were taken from 2 weeks April 2011. In many ways, this test network presents a challenging forecasting case these are all drawn from the real USA. Forecasts with all models are made at midnight every day for subsequent 47 hourly increments. Load forecasts across all 123 premises on the network are aggregated together and compared to the actual aggregate load.

\subsection{Simulation result}

Figure 3.6 Shows the comparison between the actual and predicted load for the home with different prediction algorithm technique one using FFANN, the second using FFANN with the extracted predicted appliances from FHMM, and FFANN with the extracted predicted appliances from CO. Figure 3.7 Shows the difference Error between the expected power developed by ANN or ANN_HMM or ANN_CO and the actual power. It can be noticed from Figure 3.7 that the orange graph represent the least error condition. Table 3.1, shows the estimated RMSE and NRMSE at different predictor approached used in household load forecasting through this work. It can be inferred that the approach has the least RMSE and the least NRMSE is the one used the ANN with combined with preprocessing NILM technique (HMM). 
Table 3.1 Comparison of the RMSE and NRMSE with the three predictors approach

\begin{tabular}{llll}
\hline \hline & ANN & ANN_HNN & ANN_CO \\
RMSE & 0.088693502 & 0.034189461 & 0.110849268 \\
NRMSE & 0.065156642 & 0.033566291 & 0.700878406 \\
\hline
\end{tabular}

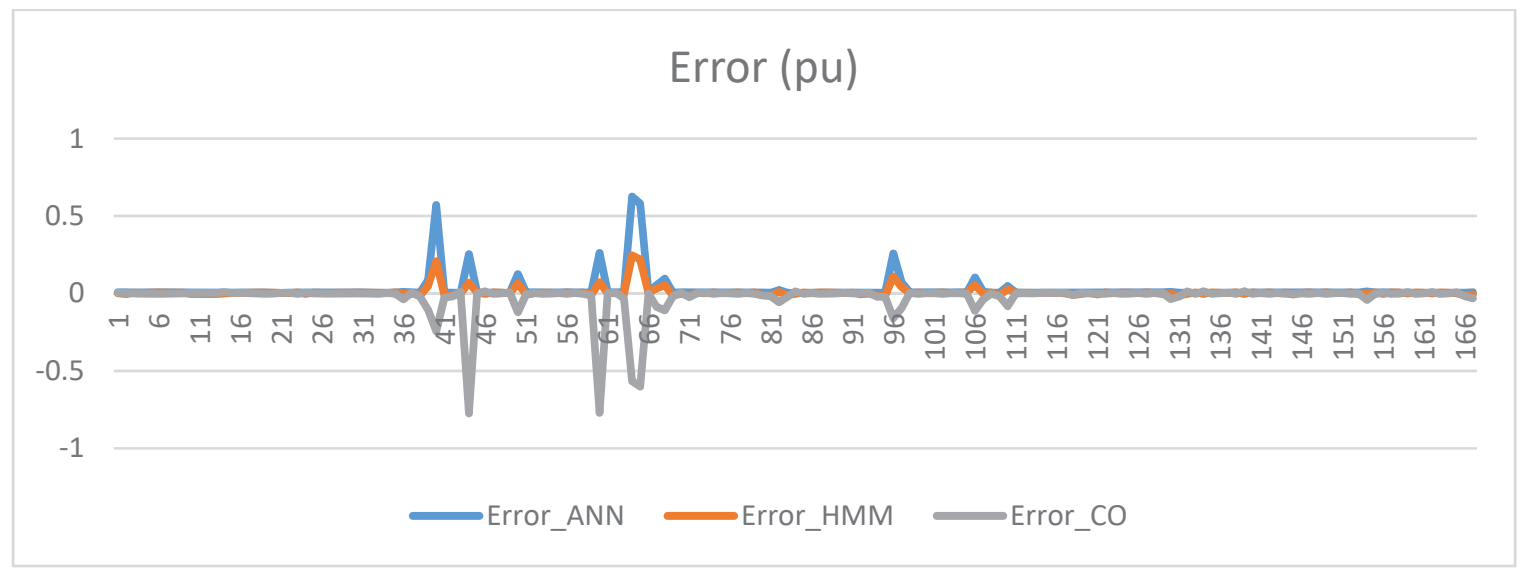

Figure 3.7 Show the difference Error point by point.

\subsection{CONCLUSION}

This work for the first time explores the potential of employing the state-of-art NILM techniques combined with ANN for household STLF under high uncertainty and volatility. The proposed mechanism provides the possibility to forecast the energy consumption conditions and to predict the home energy requirements at different times of the day or on different days of the week. The proposed approach is suitable for smart grid applications for effective demand-side management, as it can rely on matching present generation values with demand by controlling the energy consumption of appliances and optimizing their operation at the user side. 


\section{Chapter 4 Evaluation of NILM-Based Deep Learning Techniques for Efficient Demand Side Management}

The development of the current power system grid is essential to better serve the customer. Especially, with the massive expansion in the distribution network and the increase of renewable energy penetration. This development means the grid must be reliable, more flexible, and smarter. Households represent a massive portion of the grid infrastructure. The deployment of smart appliances enables the grid to be smarter through energy/demand-side management based appliances control. Therefore, Short-Term Load Forecasting (STLF) is needed to help the energy management for households' systems. The main challenge for STLF at the household's level is the high uncertainty in the load demand. In this paper, a new method for households' STLF is employed. It consists of a Feed-Forward artificial neural network (FFANN) and a pre-processing stage of Energy Disaggregation (ED) techniques. Extract the individual load pattern of household's appliances from the total load demand by the ED techniques, enlarge the training data set of the FFANN forecaster to achieve a remarkable improvement for its forecasting performance. Three deep neural network architectures have been used for energy disaggregation. 1) denoising autoencoder (DAE);2) a unique type of a recurrent neural network (RNN) entitled long short- term memory (LSTM); 3) a network that provides rectangles for the estimated demand by regression the start time, end time and average power demand (nicknamed as RECTANGLES). The proposed method outperforms the 
state of the art techniques in household load forecasting regarding RMSE, NRMSE, and MAE.

\subsection{Introduction}

The current power system is expanding, and more alternative renewable sources are emerging du one to the continuous increase in the demand sectors. As a result of the revolution in technology which leads to new load demand emerging. For example load demand with time-varying behavior, and excessive penetration for Electric Vehicle (EV) in power grid. Add all of that to the huge penetration of renewable energy which has intermittent behavior. The grid almost works near to the stability limits.

Therefore, moving towards a smart grid is essential and require more revolutions at different power system infrastructure's levels. Households load demand represents a significant portion of electrical energy consumption. Households' demand-side response (DSR) enables the active participation of the household load instead of considering it as a passive load. As a consequence, it provides a positive impact on the power system stability.

Forecasting the household load consumption is essential for residential DSR program. Precise short-term load forecasting (STLF) on the household level can significantly influence the management and the power system operations. Also, STLF is a necessary tool required in the management and control of the households' loads to enable smart consumption. The main challenge for STLF at the household's level is the high uncertainty in the load demand, due to the difficulty to expect the customer behavior. 
There is a lot of research done in the literature on load forecasting. The conventional methods are used 1). Exponential smoothing and autoregressive integrated moving average (ARIMA) which is based on time-series analysis. 2). feed-forward artificial neural networks (FFANN) and support vector machine (SVM) which is machine learning methods. A day ahead of load forecasting based on an adopted ARIMA model was offered in [116]. The prediction mechanism in this study is based on gathering the targeted day with a comparable meteorological day in the historical data. Another study offers Radial basis function (RBF) neural network is used for STLF [117]. A combination between the adaptive neural fuzzy inference system (ANFIS) and RBF neural network is provided In [118]. This study was established to regulate the forecasting by considering the real-time electricity price. A short-term load forecasting based neural network presented in [119]. It considered the current and previous of the load value as the Input to predict the consecutive load value at the next time step. The prediction of the Australian national aggregated load demand is studied based on extreme learning machines (ELM) [120]. A committed input selection strategy to work with the hybrid prediction framework based on wavelet transformation and the Bayesian neural network was presented in [121]. All the previous load forecasting techniques mentioned in literature can be concluded in three categories. In the first category, all approaches avoid the uncertainty by Clustering/classification methods which gather similar customers, days or weather to reduce the alteration of uncertainty within each cluster [122]. However, the performance is comprehensively dependent on the available data. The second category is using the aggregated historical data to cancel out the uncertainty and make it easier to predict. Also, it is effective only on an aggregated level. The third category is demanding on analyzing the data by pre- 
processing techniques to separate the normal pattern from the uncertainty and noise component. These pre-processing techniques are spectral analysis empirical mode decomposition (EMD) [123], Fourier transform [124], and wavelet analysis[125]. Therefore, this category is improper to be used in household STLF because the uncertainty represents most of the load pattern.

All the previously mentioned work for load forecasting are suitable for the aggregated level such as community or system level. There are a few numbers of studies on the literature regarding the household load forecasting. For instance, the first two trials reported for the household's demand load forecasting introduced in [126],. Wherein, a functional time series prediction technique was presented. But, it didn't use a commonly used metric to measure its performance. It used daily median absolute errors (DMAE) metric which unsuitable to be used as a benchmark for preliminary assessments. Unlike, the mean absolute percentage error (MAPE) which considered the standard metric for the load forecaster performance. Recently, a load forecasting technique using the deep artificial neural network (DANN) improves the accuracy of load forecasting for industrial customers presented in [127]. A factored conditional restricted Boltzmann machine was used for the first time for the purpose of household load forecasting. And it's performance outperforms SVM and FFANN. The long short-term memory (LSTM) which is a type of deep neural network (DNN) techniques used for household load forecasting [128]. The deep learning is prone to over-fitting issues compared with artificial neural networks [129]. However, it was expected to succeed in load forecasting as it was in other similar work such as pattern recognition. The problem was anticipated due to the presence of a high number of 
parameters and comparatively fewer data. In order to tackle the overfitting problem, a new technique called a pooling-based deep recurrent neural network (PDRNN) is presented in [130]. Nevertheless, the technique was trying to solve the over-fitting issue by enlarging the dimension of the training data.

The main drawback here that this technique is collecting the data from the neighboring household and gathering them in one pool to increase the training dataset. However, these data hard to access because of privacy. A preliminary work based on extracting load pattern by applying a Factorial hidden Markov model and combinatorial optimization [131]. These extracted data increased training data set and enhanced the load forecasting. However, the accuracy of the extractor needs more enhancement to affect the forecasting performance significantly. In this work, a new method for households' load demand forecasting is employed based on a pre-processing stage of energy disaggregation techniques and feedforward artificial neural network. The ED techniques analyze the available historical aggregated load demand to extract the individual load pattern. These extracted new data increase the training data window for the FFANN forecaster and has a preliminary predicted signature for the aggregated demand. As a consequence, a significant enhancement for its prediction performance was achieved. Three deep neural network architectures have been used for energy disaggregation. 1) denoising autoencoder (DAE); 2) a unique arrangement of a recurrent neural network (RNN) entitled long short- term memory (LSTM); 3) a network that products a rectangles for the estimated load pattern by regression the start time, the end time and the mean load demand (nicknamed by RECTANGLES). In the comparison between the proposed technique and the state of the 
art techniques in household load forecasting such as ARIMA, and SVM, the proposed method outperforms ARIMA, SVM, and FFANN regarding RMSE, NRMSE, and MAE.

\subsection{ENERGY DISAGGREGATION}

Energy disaggregation is a computational methodology for extracting appliances power demand from a single meter which records the aggregated power consumption for a single household. The research begins with the work of George Hart [132] in the mid-1980s. His primary work defined a signature taxonomy of feature. Nevertheless, he concentrated on extracting only the transitions among steady-states. Subsequent Hart's leads, many ED algorithms prepared for low-frequency data (1 Hz or less). Nevertheless, which extract a minor number of features only. Numerous examples of manually engineering feature extractors about the high frequency (sampling at $\mathrm{kHz}$ or more ), argued in the literature [133], [134]. By observation learning, humans are able to observe the appliances profile among aggregate data profile by eye. Particularly, the appliances with distinguishing signatures. Hand-engineer signature detectors for immediate difference of Gaussians (DoG) and scale-invariant feature transform (SIFT) was the main procedures to extract signatures for image classification before 2012 [135]. Nevertheless, in 2012, several procedures accomplished an outstanding performance without using the hand-engineered signature extractors. These findings obtained through the competition of ImageNet Large Scale Visual Recognition. In which, they used numerous disaggregation procedures to extract a hierarchy of signatures from the raw image.

In this work, a three adapted deep learning algorithms for energy disaggregation called DAE, RNN, and RECTANGLES have been used to extract the load demand pattern for 
every single appliance from the total Household load demand. Figure 4.1 shows The proposed household's load forecasting block diagram. The main parts of this block diagram are illustrated later in the next sections.

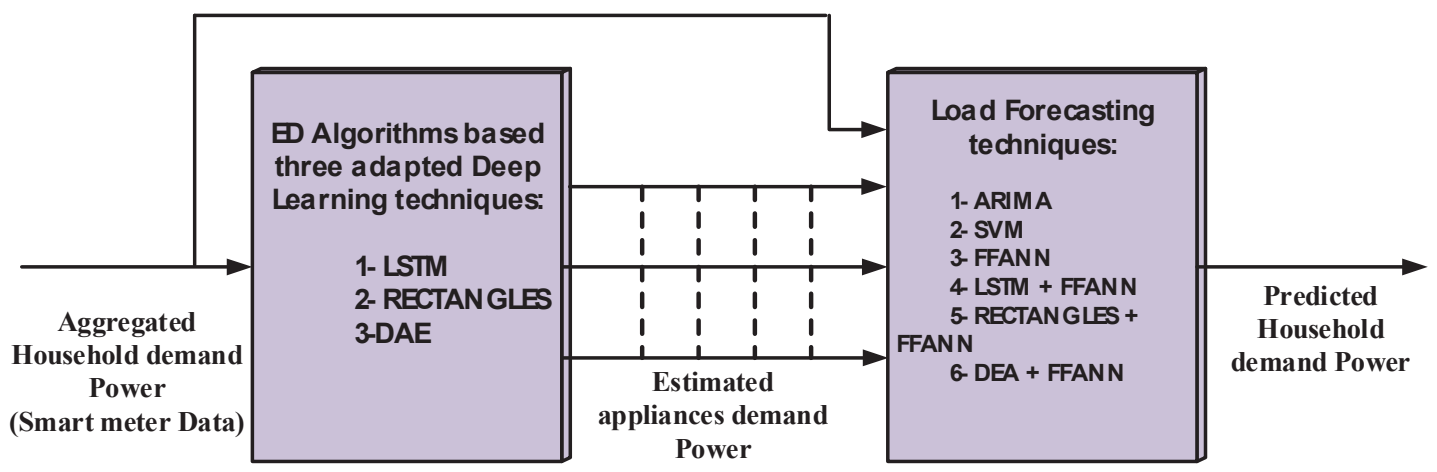

Figure 4.1 The proposed household's load forecasting block diagram.

\subsubsection{Non-Intrusive Load Monitoring Tool Kit (NILMTK)}

Non-Intrusive load monitoring toolkit is an open-source toolkit implemented based on Python which offers a significant set of libraries to support Non-Intrusive Load Monitoring (NILM), and machine learning studies [136]-[144]. In this work, the NILMTK was used for the ED purpose to improve the household's load forecasting performance. To use this toolkit, available historical data for the energy consumption of each household is required. Therefore, the data from the UK-DALE is used [145]. Which is one of the primarily available datasets released in public to assist the research of NILM. Figure 4.2 shows the block diagram of the NILMTK. And all the steps from the import of the datasets to the assessment of the disaggregation algorithms over different benchmark metrics [146]. Three disaggregation algorithms were implemented on the NILMTK for the purpose of ED. The UK-DALE dataset is the first available UK dataset. It has a record of five houses. 


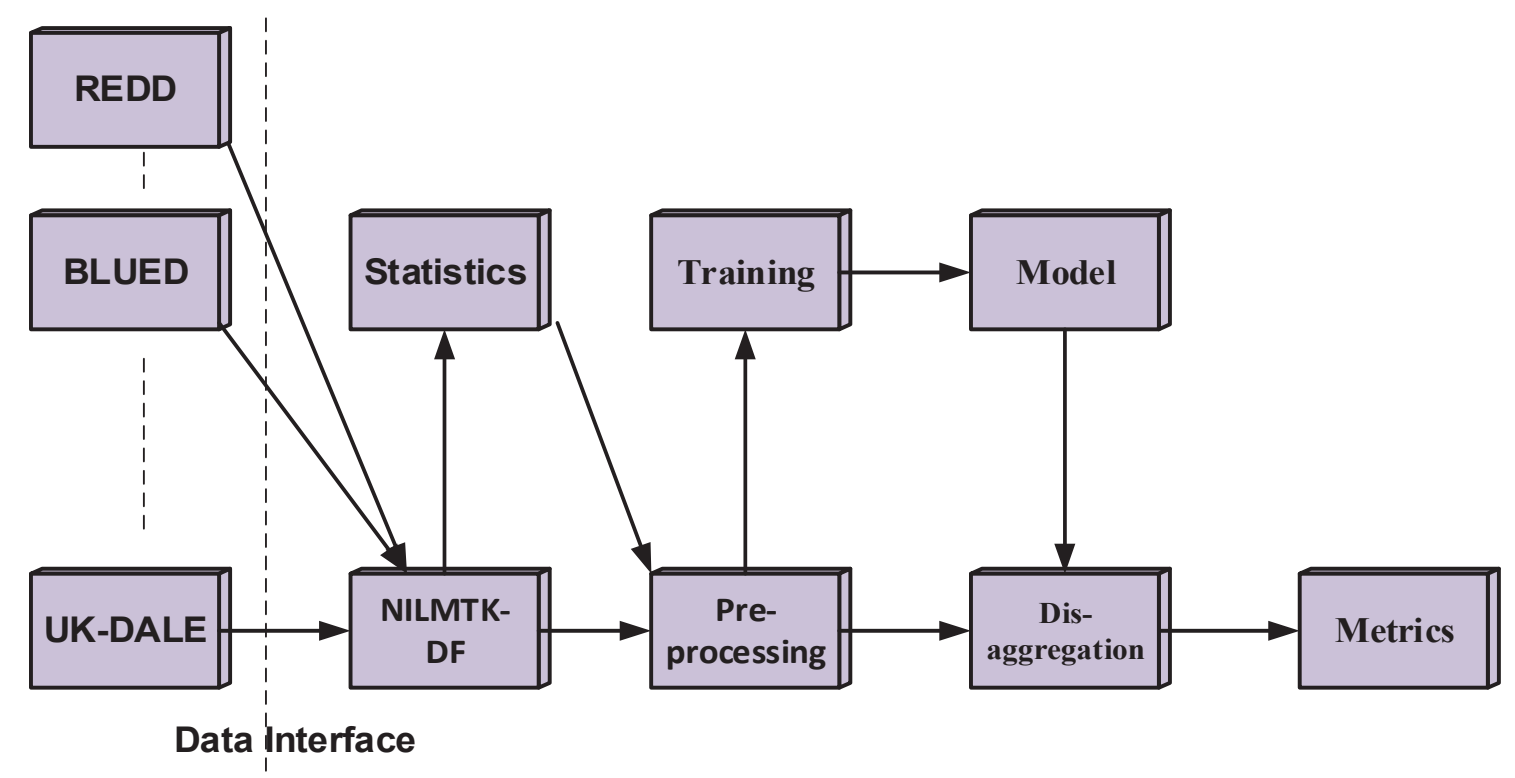

Figure 4.2 Block diagram for the NILM toolkit

But the study here focuses on two homes. One of them his historical data was available during the training of the NILM algorithm. However, the other was not seen during the training of the NILM stage.

\subsubsection{Energy Disaggregation Algorithms for Home Consumption}

\subsubsection{Denoising Autoencoder (DAE)}

An autoencoder tries to rebuild a real target profile from a noisy profile input. DAEs are trained by artificially corrupting's signal before its uses with the net's input, and using the clean profile signal as the net's target. In this work, the DAE considers the corruption profile is the power demand of the aggregated appliances power consumption. Therefore, no need to add the noise artificially. Instead, the aggregate power demand works as the noisy input to the DAE's network which used to reconstruct the clean load profile of the target appliance. Figure 4.3 show the energy disaggregation algorithm output by using DAE. 

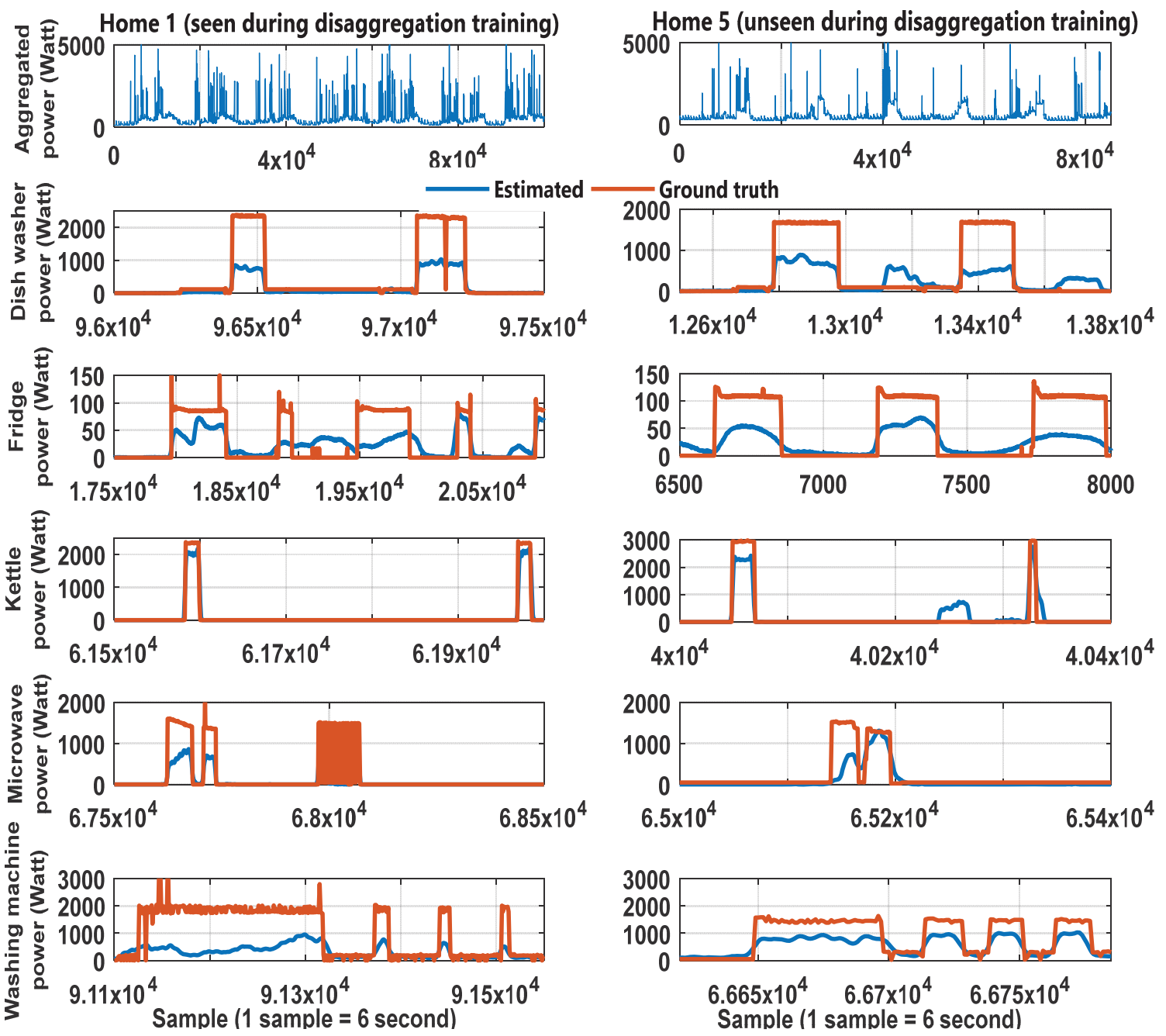

Figure 4.3 Shows the ED output performance by using DAE technique vs the ground truth data.

\subsubsection{Recurrent Neural Network (RNN)}

A recurrent neural network $(\mathrm{RNN})$ is a unique kind of ANN. In which, the relations among units construct a directed graph along a sequence. This construction enables it to show dynamic time-based performance in a time sequence. Different type of FFANN, RNNs use their internal memory to generate a sequence of inputs. Figure 4.4 show the energy disaggregation algorithm output by using RNN. 

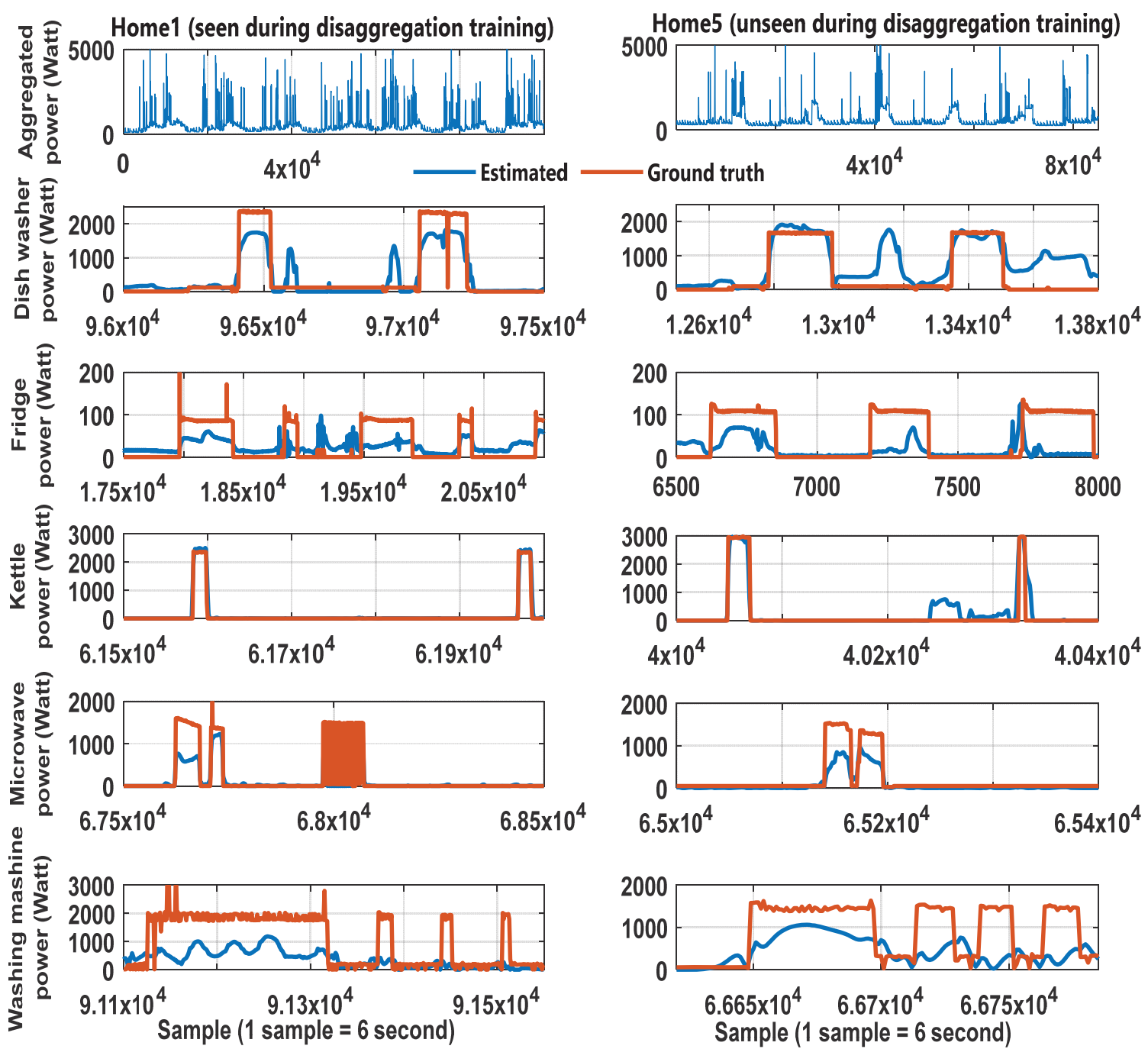

Figure 4.4 Shows the ED output performance by using LSTM technique vs the ground truth data.

\subsubsection{Regress the start time, the end time, and the mean power (RECTANGLES)}

This technique generates a rectangle around each appliance activation pattern in the aggregated data. The left side of the rectangle represents the start time of target profile, the right side represents the end time of the target profile, and the height is the mean power demand between the start and end times of the target appliances pattern. Figure 4.5 show the energy disaggregation algorithm output by using RECTANGLES. Figures 4.3, 4.4, and 4.5 are composed of two-column and six rows. The column on the left-hand 
side shows the disaggregation data of the home which his data was seen during training.

The column on the right-hand side shows the disaggregation data of the home which his data was not seen during training. The first row represents the recorded aggregated power demand for each home. From the second row to the sixth, a comparison between the ground truth data and the estimated data for five different appliances was shown. They arranged from row two to row six as follow: Dishwasher, Fridge, Kettle, Microwave, and Washing machine.

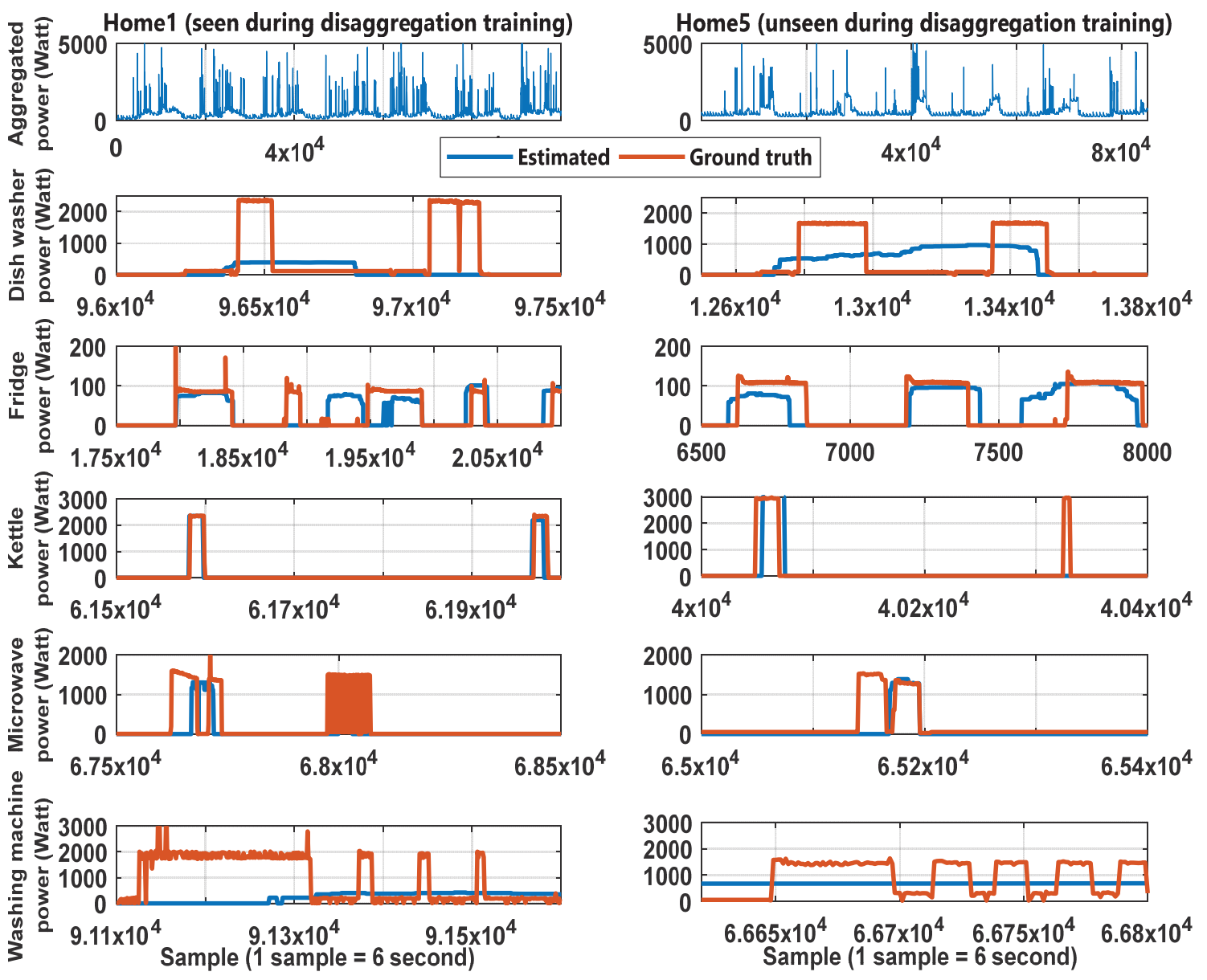

Figure 4.5 Shows the ED output performance by using RECTANGLES technique vs the ground truth data. 


\subsubsection{Disaggregation stage analysis}

In order to classify the best disaggregation algorithm performance that used in the ED stage. Seven commonly used classification metrics is used as stated by Eq 1-17:

$\mathrm{TP}=$ number of true positive

$\mathrm{FP}=$ number of false positive

$\mathrm{FN}=$ number of false-negative

$\mathrm{P}=$ number of positive in the ground truth

$\mathrm{N}=$ number of negative in the ground truth

recall $=\frac{T P}{T P+F N}$

precision $=\frac{T P}{T P+F P}$

$\mathrm{F} 1=2 \times \frac{\text { prcision } x \text { recall }}{\text { precision }+ \text { recall }}$

accuracy $=\frac{T P+T N}{P+N}$

$\mathrm{E}=$ total actual energy

$\hat{\mathrm{E}}=$ total actual energy

$y_{t}^{(i)}=$ appliance $i$ actual power at time $\mathrm{t}$

$\hat{\mathrm{y}}_{t}^{(i)}=$ appliance $i$ estimated power at time $\mathrm{t}$ 
$\bar{y}_{t}=$ aggregated actual power at time $\mathrm{t}$

relative error in total energy $=\frac{|\hat{\mathrm{E}}-E|}{\max (E, \hat{\mathrm{E}})}$

mean absolute error $=1 / T \sum_{t=1}^{T}\left|\hat{y}_{t}-y_{t}\right|$

the proportion of total energy correctly assigned $=1-\frac{\sum_{t=1}^{T} \sum_{i=1}^{n}\left|\hat{y}_{t}^{(i)}-y_{t}^{(i)}\right|}{2 \sum_{t=1}^{T} \bar{y}_{t}}$

Figure 4.6 represents the comparison between the energy disaggregation analyses for the house which its historical data was available during training.

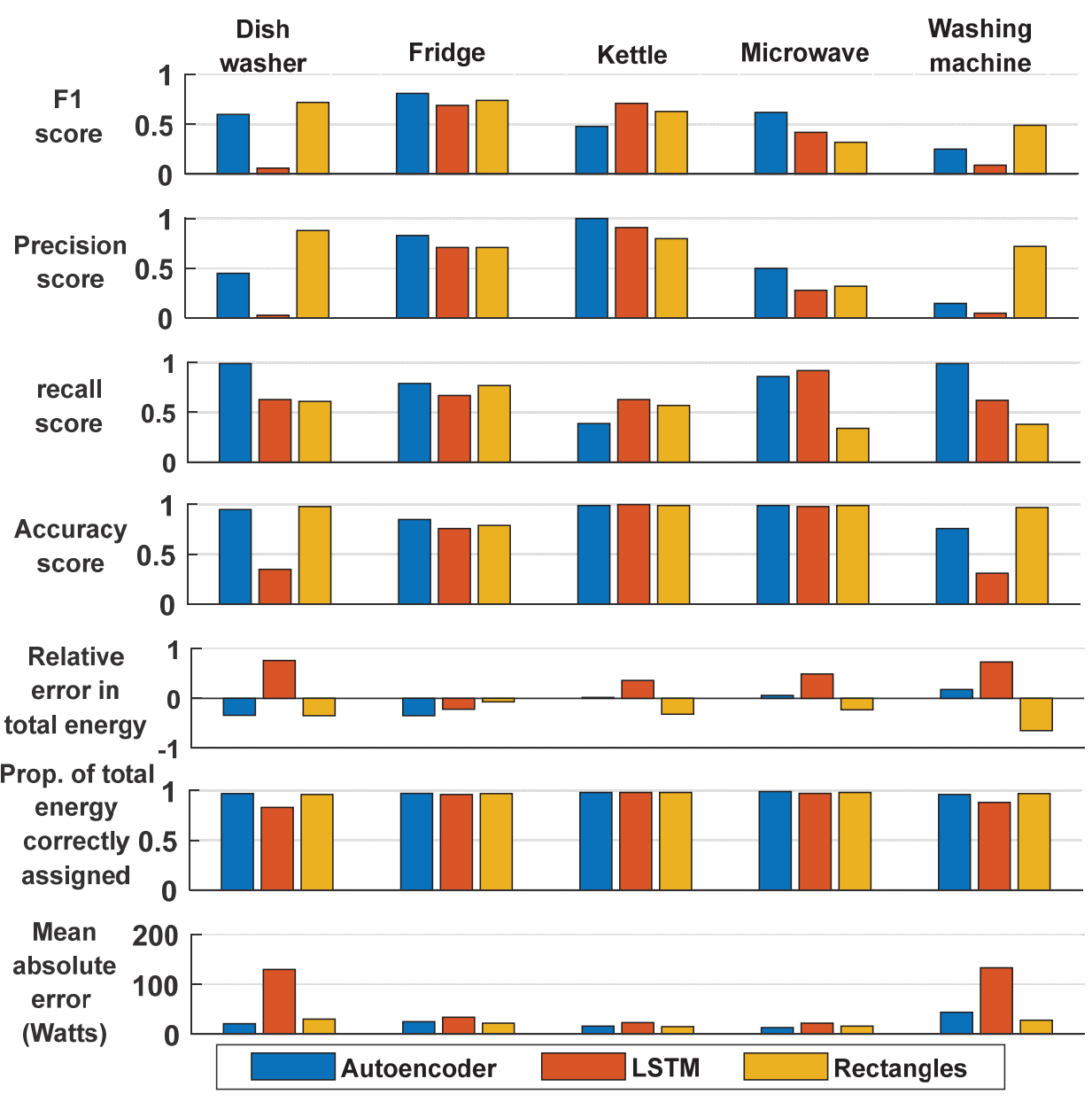

Figure 4.6 NILM performance analysis for home seen during training 
Figure 4.7 shows the comparison between the energy disaggregation analyses for the house which its historical data was unseen during training. The findings show that the denoising autoencoder outperforms LSTM, and RECTANGLES in most of the assessment metrics through the five appliances.
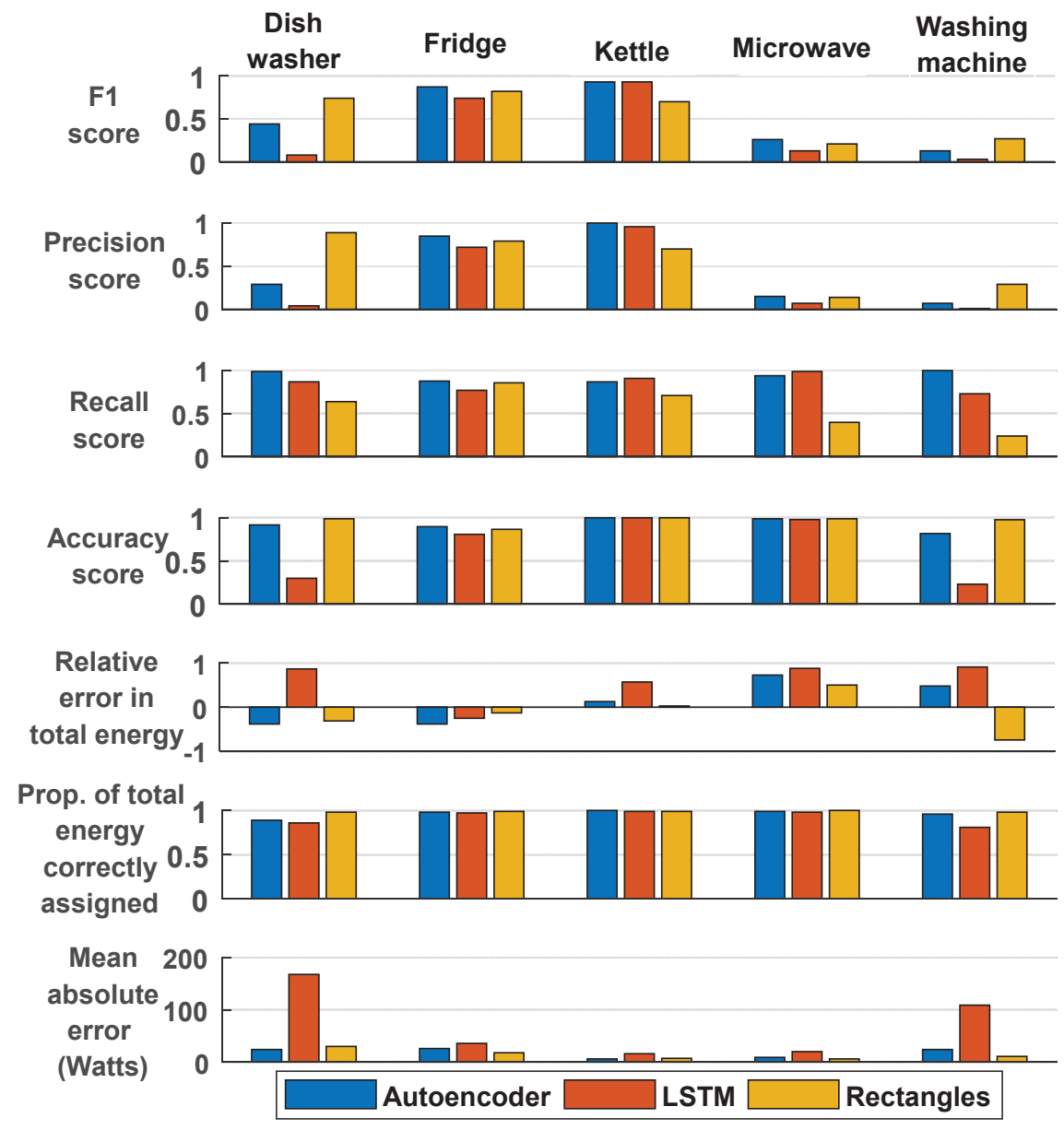

Figure 4.7 NILM performance analysis for home unseen during training

\subsection{Short-Term Load Forecasting Implementation}

An FFANN based backpropagation structure was used [147]. The Neural network contains one input layer with eleven nodes, three hidden layers, and the output layer represented by one node. The Eleven inputs represent the input layer consists of (five inputs 
from the energy disaggregation step and a six inputs from the historical aggregated demand of the house for the current and previous hours. Five inputs for the estimated power demand of the five main appliances in the house at the current hour which extracted from the energy disaggregation step (kettle, microwave, fridge, dishwasher, and washing machine). The six inputs for the current and historical consumption hours divided into two sets. Three inputs include the power demand of the current hour, the power demand for an hour before, the power demand for two hours earlier, and another three inputs composed of one for a day earlier, one for the 23 hours earlier, and one for 22 hours earlier. One output node for the expected power demand in an hour ahead.

\subsection{Simulation Result}

Figure 4.8 and Figure 4.9 show the actual load and the predicted load with a different STLF algorithm for the house, which has its data available during the ED training, and the house that has its data unavailable during ED training respectively. Three common metrics are applied to evaluate the performance of the proposed STLF method for residential grid level; root mean squared error (RMSE), normalized root mean squared error (NRMSE), and mean absolute error (MAE).

$$
\begin{aligned}
& R M S E=\sqrt{\frac{\sum_{t=1}^{N}\left(\hat{\mathrm{y}}_{t}-y_{t}\right)^{2}}{N}} \\
& N R M S E=\frac{R M S E}{y_{\max }-y_{\min }} \\
& M A E=\frac{\sum_{t=1}^{T}\left|\hat{\mathrm{y}}_{t}-y_{t}\right|}{N}
\end{aligned}
$$




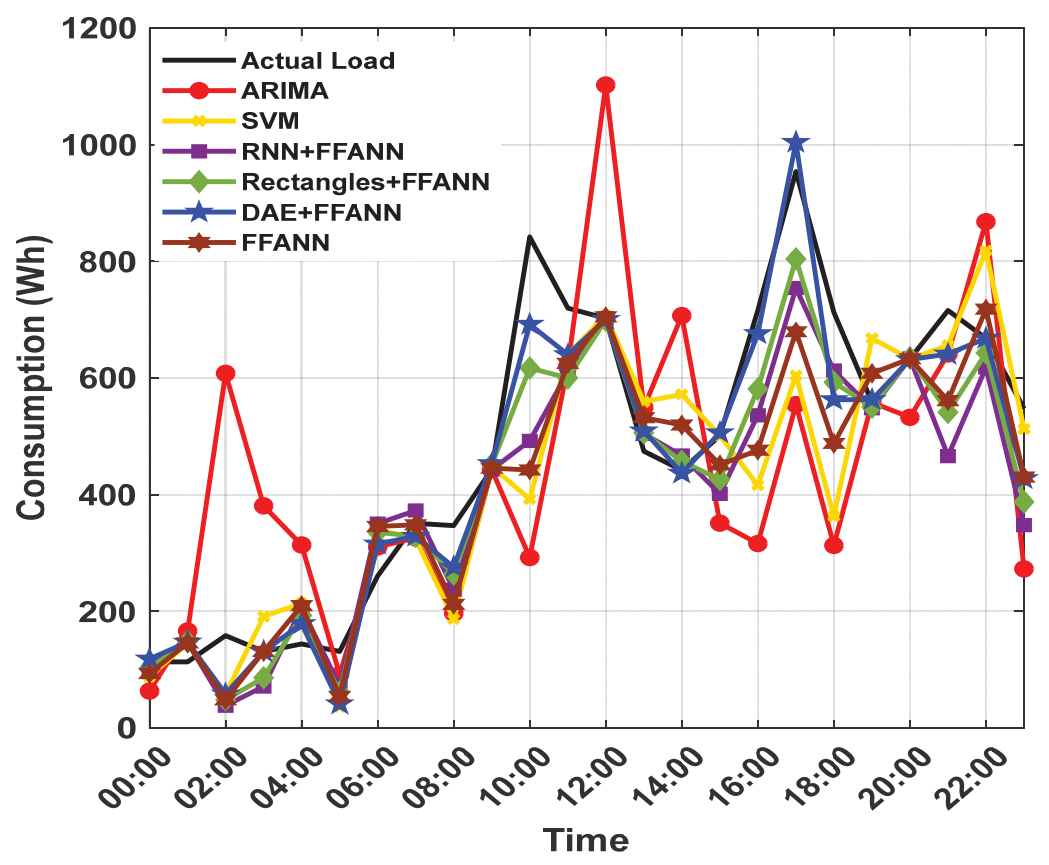

Figure 4.8 The actual and predicted load demand by different method for the home whose historical data was seen during the NILM training.

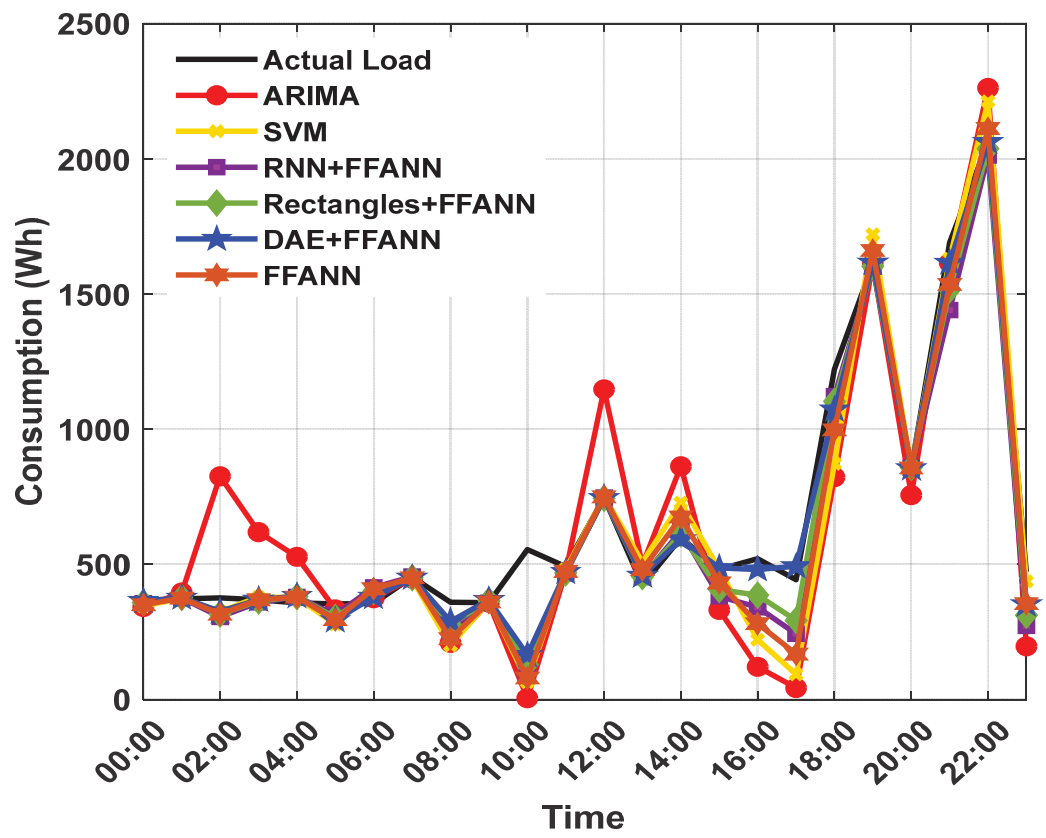

Figure 4.9 The actual and predicted load demand by different method for the home whose historical data was unseen during the NILM training. 
Table 4.1 and Table 4.2.give a comparative analysis of different performance metrics used to assess all the STLF approach used. The proposed STLF approach outperform regarding RMSE, NRMSE, and MAE with the common state of the art techniques, i.e., AIRMA, SVM, and FFANN. All the presented metrics in the tables take the averaged values across all the tested households. As illustrated, the three proposed approach (Rec + FFANN, RNN + FFANN, and DAE + FFANN) outperform ARIMA, SVM, and FFANN in all performance metrics used. Result in Table II was expected to be the worst because the data was unseen during the ED stage. However, the proposed approach shows excellent performance that means it succeeds to generalize to unseen data. The proposed (DEA + FFANN) brings $20.37 \%$ reduction in RMSE and NRMSE, $17.48 \%$ reduction of MAE. Compared with ARIMA.

Table 4.1 Load Forecasting Performance Comparison for the seen home

\begin{tabular}{llll}
\hline \hline Architecture & RMSE(kwh) & NRMSE & MAE(kwh) \\
ARIMA & 0.543 & 0.102 & 0.288 \\
SVM & 0.527 & 0.106 & 0.283 \\
FFANN & 0.508 & 0.103 & 0.274 \\
Rec + FFANN & 0.493 & 0.087 & 0.267 \\
RNN+FFANN & 0.471 & 0.086 & 0.259 \\
DEA+FFANN & 0.429 & 0.078 & 0.248 \\
\hline \hline
\end{tabular}


Table 4.2 Load Forecasting Performance Comparison for the unseen home

\begin{tabular}{llll}
\hline Architecture & RMSE(kwh) & NRMSE & MAE(kwh) \\
ARIMA & 0.553 & 0.108 & 0.289 \\
SVM & 0.528 & 0.107 & 0.289 \\
FFANN & 0.509 & 0.104 & 0.279 \\
Rec + FFANN & 0.498 & 0.095 & 0.269 \\
RNN+FFANN & 0.478 & 0.089 & 0.262 \\
DEA+FFANN & 0.440 & 0.086 & 0.238 \\
\hline \hline
\end{tabular}

\subsection{CONCLUSION}

This work explores the possibility of employing FFANN combined with a preprocessing stage of energy disaggregation for households' STLF with high uncertainty in the load pattern.

Three energy disaggregation techniques were applied and assessed for a data of two different houses; DAE, RECTANGLES, and RNN. Seven assessment metrics were utilized to benchmark the implemented energy disaggregation techniques. These analyses give a comprehensive comparison of the techniques being assessed. The proposed STLF methods; RECTANGLES + FFANN, DAE + FFANN, and RNN + FFANN outperform FFANN, SVM, and ARIMA in all three benchmark metrics. The best way used for energy disaggregation is denoising autoencoder which affected the output performance of the STLF at the residential household system level. The proposed Deep learning techniques used for ED stage are generalized even with data from an unseen home. The best approach used for energy disaggregation is denoising autoencoder which directly affected the 
performance of the STLF at residential household level. The proposed (DEA + FFANN) brings $20.37 \%$ reduction in RMSE and NRMSE, $17.48 \%$ reduction of MAE. Compared with ARIMA. 


\section{Chapter 5 Precise Short-Term Load Forecasting for Residential Demands Considering Highly Stochastic Energy Consumption}

Demand-side management has a vital role in supporting the demand response in smart grid infrastructure; the decision making of energy management in the household applications is significantly affected by the load forecasting accuracy. This work introduces an innovative methodology to enhance household demand forecasting based on energy disaggregation for Short Term Load Forecasting. This approach is constructed from FeedForward Artificial Neural Network forecaster and a pre-processing stage of energy disaggregation. This disaggregation technique extracts the individual appliances' load demand profile from the aggregated household load demand to increase the training data window for the proposed forecaster. These proposed algorithms include two benchmark disaggregation algorithms; Factorial Hidden Markov Model (FHMM), Combinatorial Optimization in addition to three adopted Deep Neural Network, long short- term memory (LSTM), Denoising Autoencoder, and a network which regress start time, end time, and average power. The proposed load forecasting approach outperformed the currently available state of the art techniques, namely root mean square error (RMSE), normalized root mean square error (NRMSE), and mean absolute error (MAE).

\subsection{Introduction}

As mentioned, the innovations at different power system infrastructure levels facilitate the integration of new smart grid ideas. However, new architectures of the smart grid add extra burden on the grid regarding complexity and uncertainty. As a result of the increased penetration of renewable energy, Electric Vehicles (EVs), and time-varying loads in the 
distribution system, the grid will be vulnerable to unusual, challenging experiences for utility-customer interactions. Household loads represent a significant percentage of electrical energy consumption. The households' demand-side response (DSR) enables active participation of these loads in the grid enhancing power system stability.

Consequently, the forecasting of household energy consumption is crucial for the household DSR program. Precise short-term load forecasting (STLF) has a significant effect on the accuracy of the household DSR. However, STLF is challenging at this level of the grid due to uncertainty and volatility in load consumption originating from customer behavior, which is too stochastic to predict.

Common techniques such as exponential smoothing, autoregressive integrated moving average (ARIMA) based time-series analysis, support vector machine (SVM), and feedforward artificial neural networks (FFANN) based machine learning have been used in the literature to achieve good STLF forecasting.

An adopted ARIMA model for a day ahead load forecasting was presented in [148], in which the forecasting technique is based on grouping the targeted day with similar meteorological days in historical data. A radial basis function (RBF) neural network was used for STLF in [149]. An adaptive neural fuzzy inference system (ANFIS) was combined with an RBF neural network to adjust the forecasting by taking into consideration real-time electricity prices [150]. A neural network-based predictor for STLF was presented in [151]. The latter uses the load values of the current and previous time steps as inputs to predict the load value at the subsequent time step. A forecaster for the total load of the Australian national energy market was based on an ensemble of extreme learning machines (ELMs) 
is suggested in [152]. A committed input choice structure to work with the hybrid prediction framework using the Bayesian neural network and wavelet transformation was introduced in [153].

Based on the current state of the art, the procedures used for load forecasting can be classified into three categories. The first is to evade the uncertainty by clustering/classification techniques that gather comparable customers, days or weather in the hope of decreasing the variance of uncertainty within each cluster [154]. However, the accuracy of this technique is heavily dependent on the amount of available data. The second category is using the aggregated smart metering data to cancel out the uncertainty. Therefore, the aggregated load exhibits typically regular patterns and more accessible to predict. However, the accuracy of this technique is heavily dependent on an aggregated level of data. The third category is separating the regular pattern from the other component of load profile such as uncertainty and noise by pre-processing techniques, mostly spectral analysis such as empirical mode decomposition (EMD) [155], Fourier transforms[156], and wavelet analysis[157]. These techniques are unsuitable for the household load forecasting due to the high uncertainty proportion of the load pattern.

All these previous techniques are appropriate for higher grid levels, such as community or system levels. However, there are few works done in the literature on STLF at the household level. In [158, 159], a functional time series forecasting approach was presented. However, it used a daily median absolute error (DMAE) which is not the commonly used performance metric. Therefore, it is improper for use as a benchmark for preliminary assessments. Mean absolute percentage error (MAPE) is the standard metric used to assess 
forecaster performance. Recently, a deep artificial neural network (DANN) used in household load forecasting [158]. The DANN technique used is a factored conditionally restricted Boltzmann machine. The latter improve performance rather than support vector machine and artificial neural network. Another deep neural network (DNN) approach called long short-term memory (LSTM) was used in [160]. Although the high expectation in the forecasting community, the current state of the art indicates that deep learning is more prone to over-fitting compared with artificial neural networks [161]. This issue is expected due to the existence of more parameters and relatively fewer data. For that reason, another work based on a pooling-based deep recurrent neural network (PDRNN) was proposed in [162] to tackle the overfitting issue. However, the procedure was an attempt to tackle the over-fitting issue by increasing the training data window dimension which is the historical data of the neighbors to the household system under study. The main drawback here is that the PDRNN method pools the data of the neighboring smart meters to enlarge the training widow dataset which most probably is unavailable for privacy concerns.

In this work, an innovative methodology for STLF of household load demand is developed and employed. This approach is constructed from Feed Forward Artificial Neural Network (FFANN) and a pre-processing Stage of Energy Disaggregation (SOED) based on Data Mining Algorithms (DMA). This SOED extracts the individual appliances' load demand profile from the aggregated household load demand to increase the training data window for the FFANN forecaster. These DMA include two bench-mark disaggregation algorithms (Factorial Hidden Markov Model (FHMM), Combinatorial 
Optimization) and three adopted Deep Neural Network (Long Short-Term Memory (LSTM), Denoising Autoencoder (DAE), and a network which regress start time, end time, and average power (RECTANGLES)). The proposed load forecasting approach outperforms the current state of the art techniques such as ARIMA, SVM, and FFANN regarding RMSE, NRMSE, and MAE.

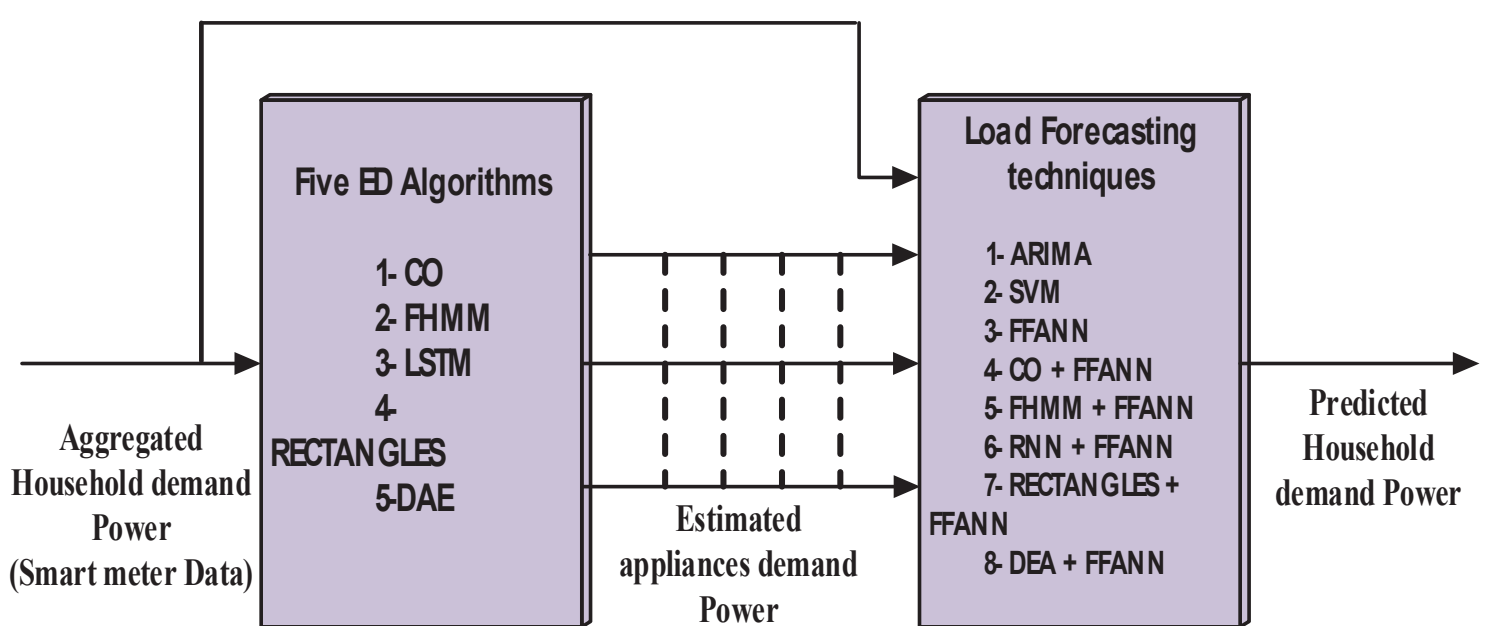

Figure 5.1 Block diagram for the proposed household load forecasting approach.

\subsection{Energy Disaggregation}

Energy disaggregation (ED) is a computational approach for predicting the individual appliance's power demand from a single meter which measures the aggregated power demand. George Hart starts this research in the mid-1980s [163], [164]. His earliest research defined a signature taxonomy of features. Nevertheless, his concentration was on extracting only transitions between steady-states. Consequent Hart's clues, several ED procedures prepared for low-frequency data $(1 \mathrm{~Hz}$ or slower $)$ only to extract a minor number of features. There are numerous instances in the literature of manual feature extractors regarding the high-frequency sampling at kHz or even MHz. [165], [166]. Handengineer feature extractors, for instance, Difference of Gaussians (DoG) and scale- 
invariant feature transform (SIFT), was the leading method to mine features for image classification before 2012 [167]. However, in 2012, through the competition of ImageNet Large Scale Visual Recognition, diverse procedures achieved exceptional performance and did not use hand-engineered feature detectors. As an alternative, they used a number of disaggregation algorithms that automatically learned to extract a hierarchy of features from the raw image. In this paper, we will use five data mining algorithms for ED called CO, FHMM, DAE, LSTM, and RECTANGLES to extract the power demand profile for individual appliances from the main aggregated household power demand. Figure 5.1 shows the block diagram of the whole proposed system. The comprehensive illustration of these algorithms presented later in this section. To use ED to enhance household forecasting performance, the energy consumption of each household must be available. Therefore, the dataset from the UK-DALE was used [168]. Which is one of the first publicly available datasets collected essentially to support research on ED. It has a record of five houses. In our work, we will focus the study on only two houses. One his recorded data was available during the training of the ED algorithm. However, the other was not seen during the training stage of the ED algorithm. Those five ED algorithms were implemented based on a toolkit called NILMTK [169]. The code is written in Python which offers a massive set of libraries supporting both machine learning and ED algorithms.

\subsubsection{Data Mining Disaggregation algorithms}

Five data mining disaggregation algorithms were used in this work. Two bench-mark disaggregation algorithms, Factorial Hidden Markov Model (FHMM) and Combinatorial Optimization, were utilized. Moreover, three adapted deep neural network architectures 
have been used for ED; 1) an exceptional form of a recurrent neural network (RNN) called long short- term memory (LSTM); 2) a network that produces rectangles for the estimated demand by regression of the start time, end time and average power demand (nicknamed by RECTANGLES); and 3) denoising autoencoder (DAE). The full illustration of these algorithms is presented later in this section.

\subsubsection{Combinatorial Optimization (CO)}
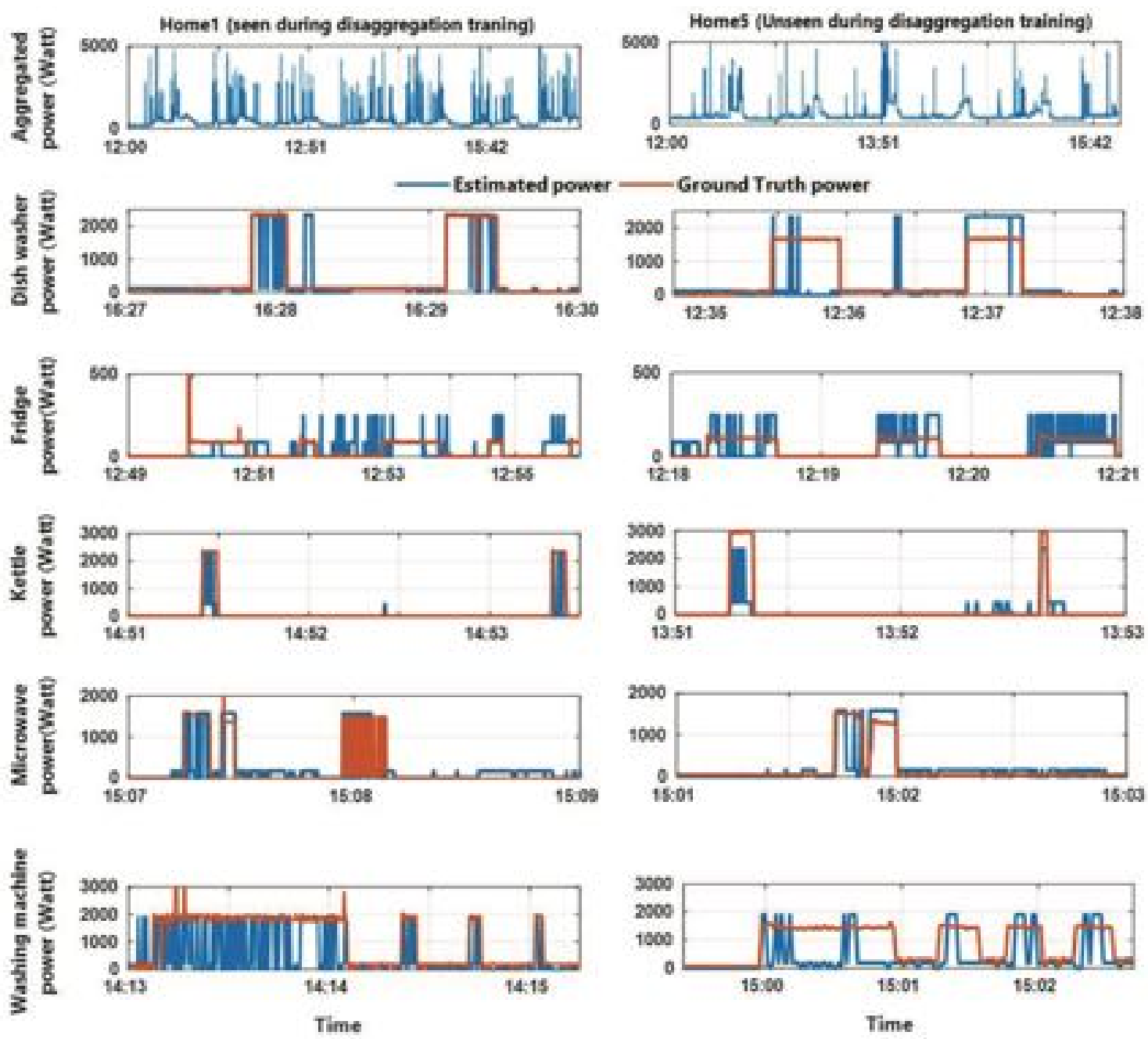

Figure 5.2 Shows the energy disaggregation algorithm output by using the $\mathrm{CO}$ technique. (a) Aggregated power consumption for the home; (b) Dish Washer estimated and Ground truth power demand; (c) Fridge estimated and Ground truth power demand; (d) Kettle estimated and Ground truth power demand; (e) Microwave estimated and Ground truth power demand; (f) Washing machine estimated and Ground truth power demand 
Optimization methods necessitate the presence of appliance signature libraries with all possible groupings of power demands of the appliances it desires to disaggregate. If we include the gatherings of all the connected appliances in a house, then this optimization approach is called brute-force. However, due to memory limitations, as stated in [170]. Brute-force methods are difficult to be applied in an embedded system. Therefore, the load identification requires the definition of an objective function and its minimization. Considering the aggregate data $\bar{x}$ and an appliance set $=\left[x_{1}, \ldots \ldots \ldots \ldots, x_{N}\right]$, the problem is formulated as [170].

$$
\min _{1 \leq n \leq N}\left\|\bar{x}-\sum_{n=1}^{N} x_{n}\right\|
$$

Combinatorial Optimization is the most critical algorithm in this domain which minimizes the difference between the sum of the measure aggregate power and predicted appliance power [171]. Hart, in [172], used this technique. The computational complexity is $o\left(K^{N} T\right)$, where $\mathrm{K}$ is the number of appliance states, $\mathrm{N}$ the number of appliances and $\mathrm{T}$ the number of times slices used in the implementation. Consequently, the optimization approaches address mainly disaggregation for the most power-hungry devices. Reference [173] discusses the two commonly cited disadvantages of this approach which are the decreasing of accuracy with the number of appliances and level of noise. Figure 5.2 shows the output result of the $\mathrm{CO}$ energy disaggregation algorithm. The figure divided into twocolumn. The left-hand side (LHS) column has the analysis for the home whose data was available during the training of the disaggregation algorithm. The right-hand side (RHS) column has the analysis for the home whose data was not available during the training of the disaggregation algorithm. The figure has six rows described as follows: (a) Aggregated 
power consumption for the home; (b) comparison between the estimated and Ground truth power demand for the Dish Washer; (c) comparison between the estimated and Ground truth power demand for the Fridge; (d) comparison between the estimated and Ground truth power demand for the Kettle; (e) comparison between the estimated and Ground truth power demand for the Microwave; (f) comparison between the estimated and ground-truth power demand for the washing machine

\subsubsection{Factorial Hidden Markov Model (FHMM)}

FHMM belongs to the group of Temporal Graphical Models, which is a class of probabilistic models. Such models have been applied previously to many real-world problems such as speech recognition. The most direct demonstration of sequence data is through the use of a Markov chain, which is a sequence of discrete variables. The hidden Markov model (HMMs) which is a statistical tool controls the state transitions of devices. Each variable is defined by its real power consumption in addition to other useful information such as duration of the on and off periods and time of use during the day/week. Thereby, at an instant of time $\mathrm{t}$ of a period $\mathrm{T}, \mathrm{t} \in \mathrm{T}$, the aggregate consumption is $\bar{x}(t)$ and needs to be broken down to the number of appliances $z_{t}^{n}$, where $t \in \mathrm{T}$ and $\mathrm{n} \in \mathrm{N}$ with $\mathrm{N}$ the number of appliances. The value of each device $z_{t}^{n}$ at any time corresponds to one of the K states of the trained model of the appliances[170]. The mathematical representation of an HMM represented by equation 2 through 6 [170]. The behavior of an HMM can be defined entirely and inferred by three parameters. First, the probability of each state of the hidden variable at the time $t$ can be represented by the vector $\pi$ such that 


$$
\pi_{K}=\rho\left(z_{t}=k\right)
$$

Second, the transition probabilities from state $i$ at $t$ to state $j$ at $t+1$ can be represented by the matrix A such that,

$$
A_{i, j}=\rho\left(z_{t+1}=j \mid z_{t}=i\right),
$$

Third, the emission probabilities for $\mathrm{x}$ are described by a statistical function with a parameter $\emptyset$ which is commonly assumed to be Gaussian distributed such that,

$$
x_{t} \mid z_{t}, \emptyset \sim \mathcal{N}\left(\mu_{z_{t}}, \tau_{z_{t}}\right) \text {, }
$$

Where $\emptyset=\{\mu, \tau\}$, and $\mu_{z_{t}}, \tau_{z_{t}}$ are the mean and precision of a state's Gaussian distribution. Finally, Equations 2, 3, 4 can be used to compute the joint likelihood of an HMM:

$$
\rho(x, z \mid \theta)=\rho\left(z_{t} \mid \pi\right) \prod_{t=2}^{T} \rho\left(z_{t+1} \mid z_{t}, A\right) \prod_{t=1}^{T} \rho\left(x_{t} \mid z_{t}, \varnothing\right),
$$

Where the set of all model parameters which must be found for each appliance during the training phase is represented by $\theta=\pi, A, \emptyset$. Therefore, when applying an HMM for Energy Disaggregation, it is needed to tune the $\theta$ parameters for each appliance during the training phase and afterward, given a sequence of the power signal $\bar{x}$ to find the optimal sequence of discrete states z. Their ability to handle daily operation consumption and the information about the state transition of devices makes them a suitable solution for the problem. The complexity of the disaggregation using HMMs is $O\left(K^{2} T\right)$, where $\mathrm{K}$ is the number of states of all the appliances and $\mathrm{T}$ is the number of the time slices, i.e., how many times the algorithm is required to be applied [170]. As it is shown, the complexity is 
exponential with regard to the number of appliances, while re-training is needed when a new group of appliances is added [174].

The HMMs were used for appliance load recognition, and it was also shown that they are useful in the field of ED [175]. Finally, HMMs are used to disaggregate an energy signal using a generalized appliance model, and as a result, it was possible to extract the consumption of individual devices without any manual labeling [173]. Nevertheless, the author uses low-frequency smart meter data because of the lack of high-frequency data and smart metering infrastructure supporting such high rates. Although the HMM is a powerful technique, the method for the inference of hidden states is often affected by local minima [176]. To overcome this limitation, variants of HMMs are used such as the Factorial HMM (FHMM). The concept is that the output is an additive function of all the hidden states. In the model, each observation is dependent upon multiple unknown variables [177]. Likewise, the joint likelihood of an FHMM as stated in [31] is computed by,

$$
\begin{gathered}
\rho\left(x^{(1: N)}, z \mid \theta\right)= \\
\prod_{n=1}^{N} \rho\left(z_{t}^{(n)} \mid \pi\right) \prod_{t=2}^{T} \prod_{n=1}^{N} \rho\left(z_{t+1}^{(n)} \mid z_{t}^{(n)}, A\right) \prod_{t=1}^{T} \rho\left(x_{t} \mid z_{t}^{(1: N)}, \emptyset\right),
\end{gathered}
$$

Where 1: $\mathrm{N}$ symbolizes a sequence of appliances $1, \ldots \mathrm{N}$. However, the computational complexity of both learning and disaggregating is more significant for FHMMs compared to HMMs. This is due to the conditional dependence of the Markov chains. Figure 5.3. Shows the output result of the FHMM energy disaggregation algorithm. The figure divided into two-column. The LHS column has the analysis for the home whose data was available during the training of the disaggregation algorithm. 

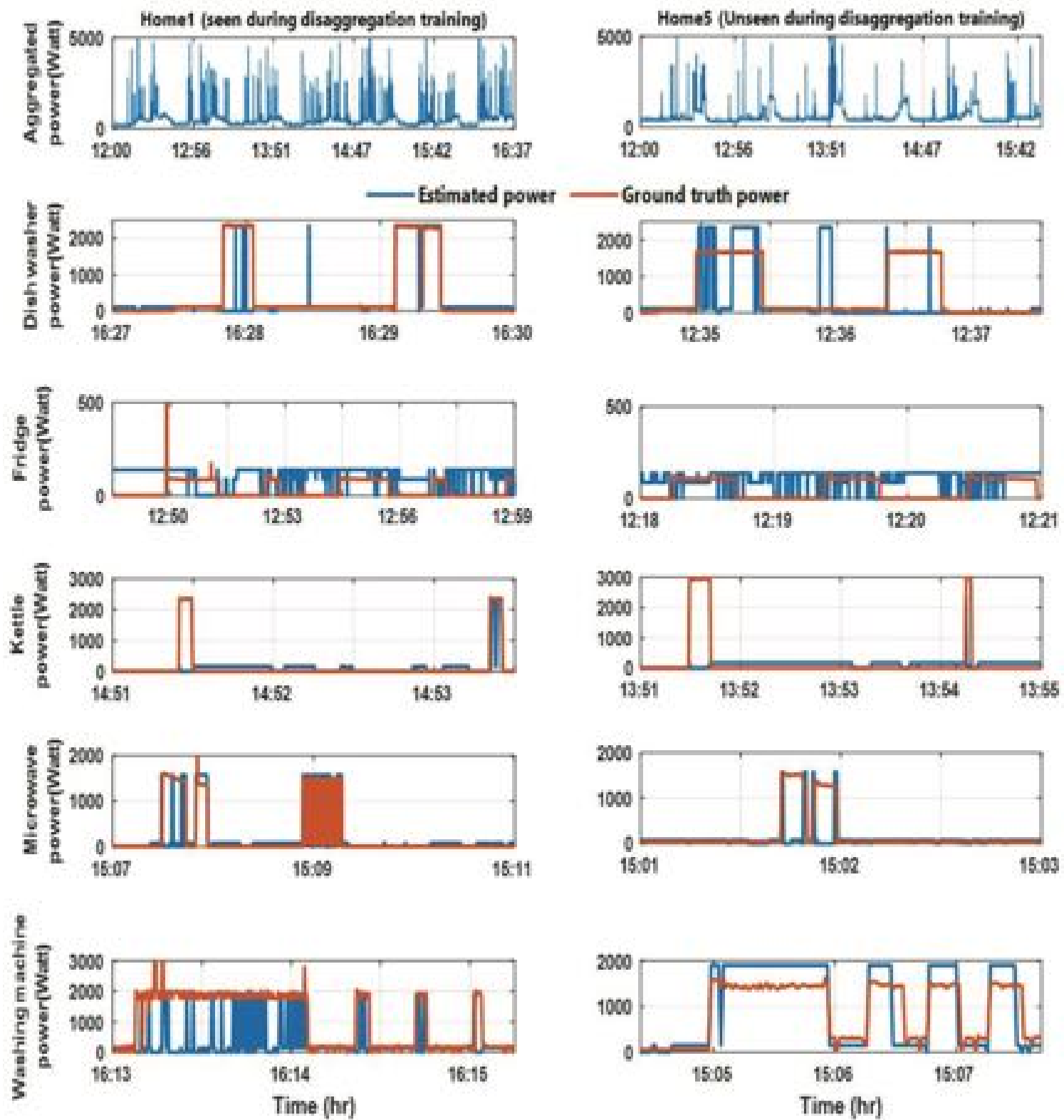

Figure 5.3 Shows the energy disaggregation algorithm output by using the FHMM technique. (a) Aggregated power consumption for the home; (b) Dish Washer estimated and Ground truth power demand; (c) Fridge estimated and Ground truth power demand; (d) Kettle estimated and Ground truth power demand; (e) Microwave estimated and Ground truth power demand; (f) Washing machine estimated and Ground truth power demand

The right-hand side (RHS column) has the analysis for the home whose data was not available during the training of the disaggregation algorithm. The figure has six rows 
described as follows: (a) Aggregated power consumption for the home; (b) comparison between the estimated and Ground truth power demand for the Dish Washer; (c) comparison between the estimated and ground-truth power demand for the fridge; (d) comparison between the estimated and ground-truth power demand for the kettle; (e) comparison between the estimated and Ground truth power demand for th microwave; (f) comparison between the estimated and Ground truth power demand for the washing machine.

\subsubsection{Denoising Autoencoder (DAE)}

It is an autoencoder, which attempts to reconstruct a clean target from noisy input. DAEs are typically trained by artificially corrupting a signal before it goes into the net's input, and using th Clean signal as the net's target. In ED, we consider corruption as being the power demand from the other appliances. Therefore, we do not add noise artificially. Instead, we use the aggregate power demand as the (noisy) input to the net and ask the net to reconstruct the clean power demand of the target appliance. Figure 5.4 shows the output result of the DAE energy disaggregation algorithm. The figure divided into two-column. The LHS column has the analysis for the home whose data was available during the training of the disaggregation algorithm. The right-hand side (RHS column) has the analysis for the home whose data was not available during the training of the disaggregation algorithm. The figure has six rows described as follows: (a) aggregated power consumption for the home; (b) comparison between the estimated and ground-truth power demand for the dishwasher; (c) comparison between the estimated and ground-truth power demand for the fridge; (d) comparison between the estimated and ground-truth power demand for the 
kettle; (e) comparison between the estimated and ground-truth power demand for the microwave; (f) comparison between the estimated and ground-truth power demand for the washing machine.
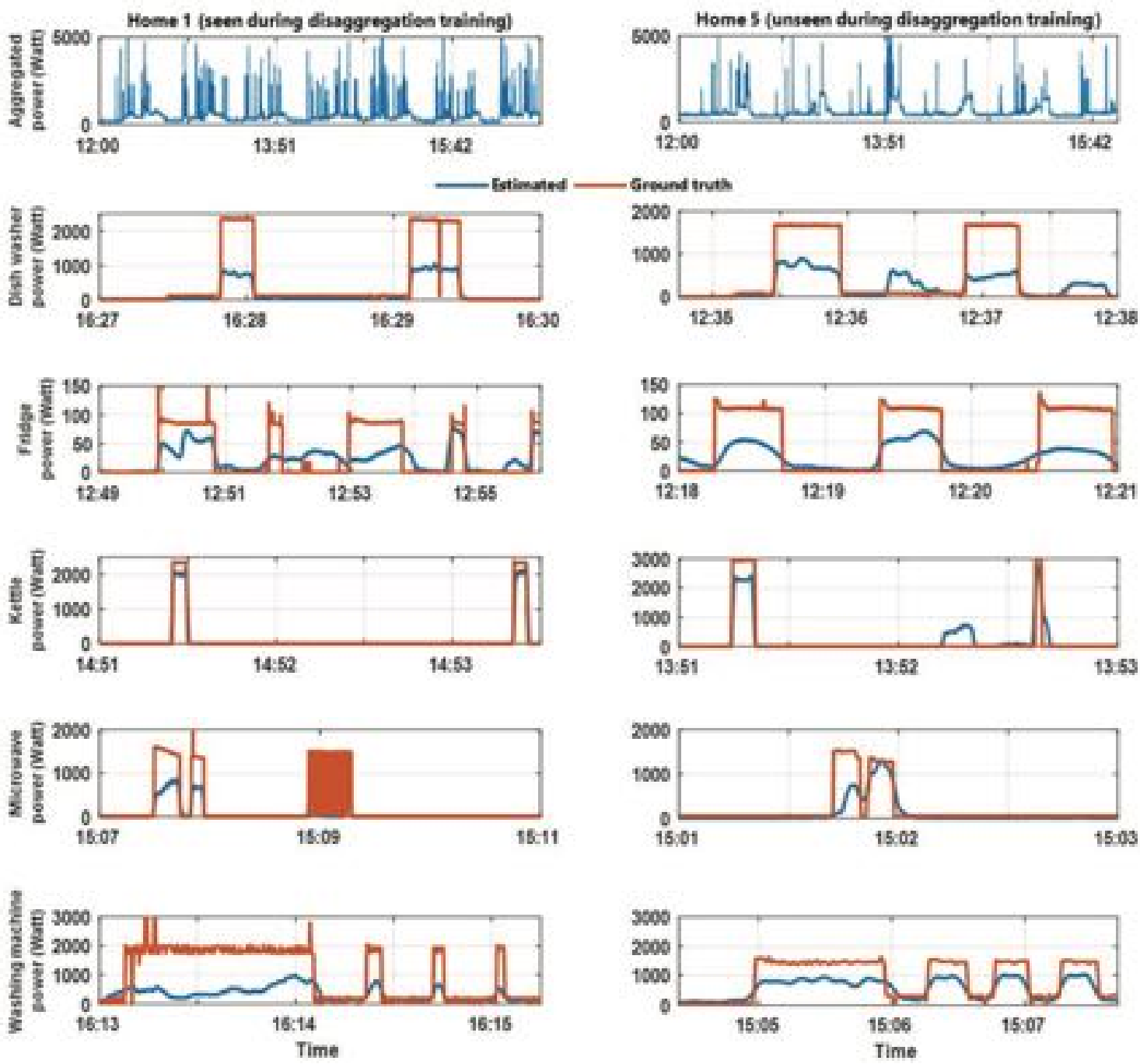

Figure 5.4 Shows the energy disaggregation algorithm output by using the DAE technique. (a) Aggregated power consumption for the home; (b) Dish Washer estimated and Ground truth power demand; (c) Fridge estimated and Ground truth power demand; (d) Kettle estimated and Ground truth power demand; (e) Microwave estimated and Ground truth power demand; (f) Washing machine estimated and Ground truth power demand 


\subsubsection{Regress start time, end time, and average power (RECTANGLES)}

This algorithm draws a rectangle around each appliance activation in the aggregate data where the left side of the rectangle is the start time, the right side is the end time, and the height is the average power demand of the appliance between the start and end times
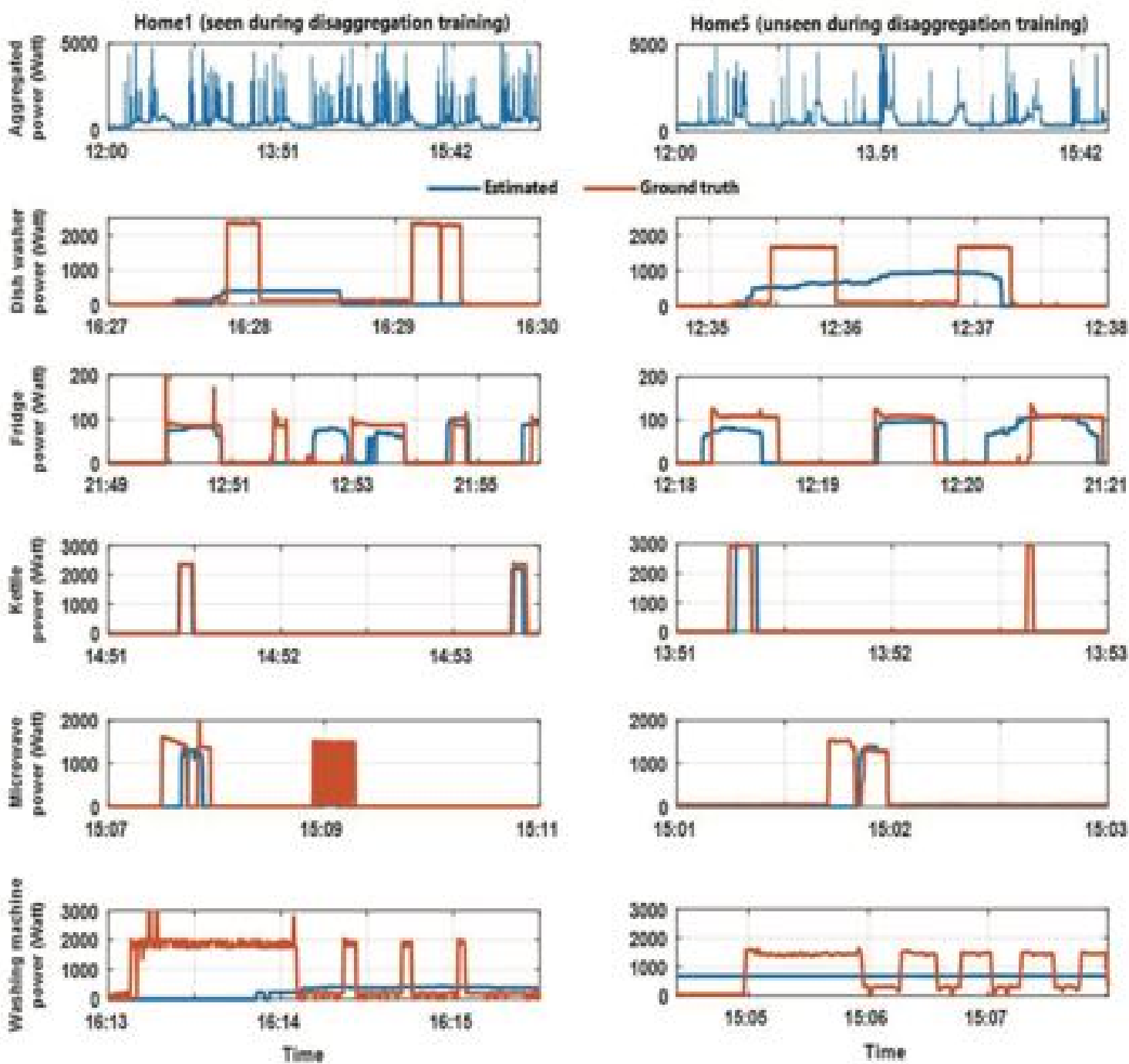

Figure 5.5 Shows the energy disaggregation algorithm output by using the DAE technique. (a) Aggregated power consumption for the home; (b) Dish Washer estimated and Ground truth power demand; (c) Fridge estimated and Ground truth power demand; (d) Kettle estimated and Ground truth power demand; (e) Microwave estimated and Ground truth power demand; (f) Washing machine estimated and Ground truth power demand 
Figure 5.5 shows the output result of the DAE energy disaggregation algorithm. The figure divided into two-column. The LHS column has the analysis for the home that its data was available during the training of the disaggregation algorithm. The right-hand side (RHS column) has the analysis for the home whose data was not available during the training of the disaggregation algorithm. The figure has six rows described as follows: (a) aggregated power consumption for the home; (b) comparison between the estimated and ground-truth power demand for the dishwasher; (c) comparison between the estimated and ground-truth power demand for the fridge; (d) comparison between the estimated and ground-truth power demand for the kettle; (e) comparison between the estimated and ground-truth power demand for the microwave; (f) comparison between the estimated and ground-truth power demand for the washing machine.

\subsubsection{Recurrent Neural Network (RNN or LSTM)}

A recurrent neural network $(\mathrm{RNN})$ is a type of artificial neural network where relations between units form a directed graph along a sequence. This allows it to exhibit dynamic temporal behavior in a time sequence. Different from feedforward neural networks, RNNs can use their internal memory to process sequences of inputs. Figure 5.6 shows the output result of the LSTM energy disaggregation algorithm. The figure is divided into two columns. The LHS column has the analysis for the home whose data was available during the training of the disaggregation algorithm. The right-hand side (RHS column) has the analysis for the home whose data was not available during the training of the 

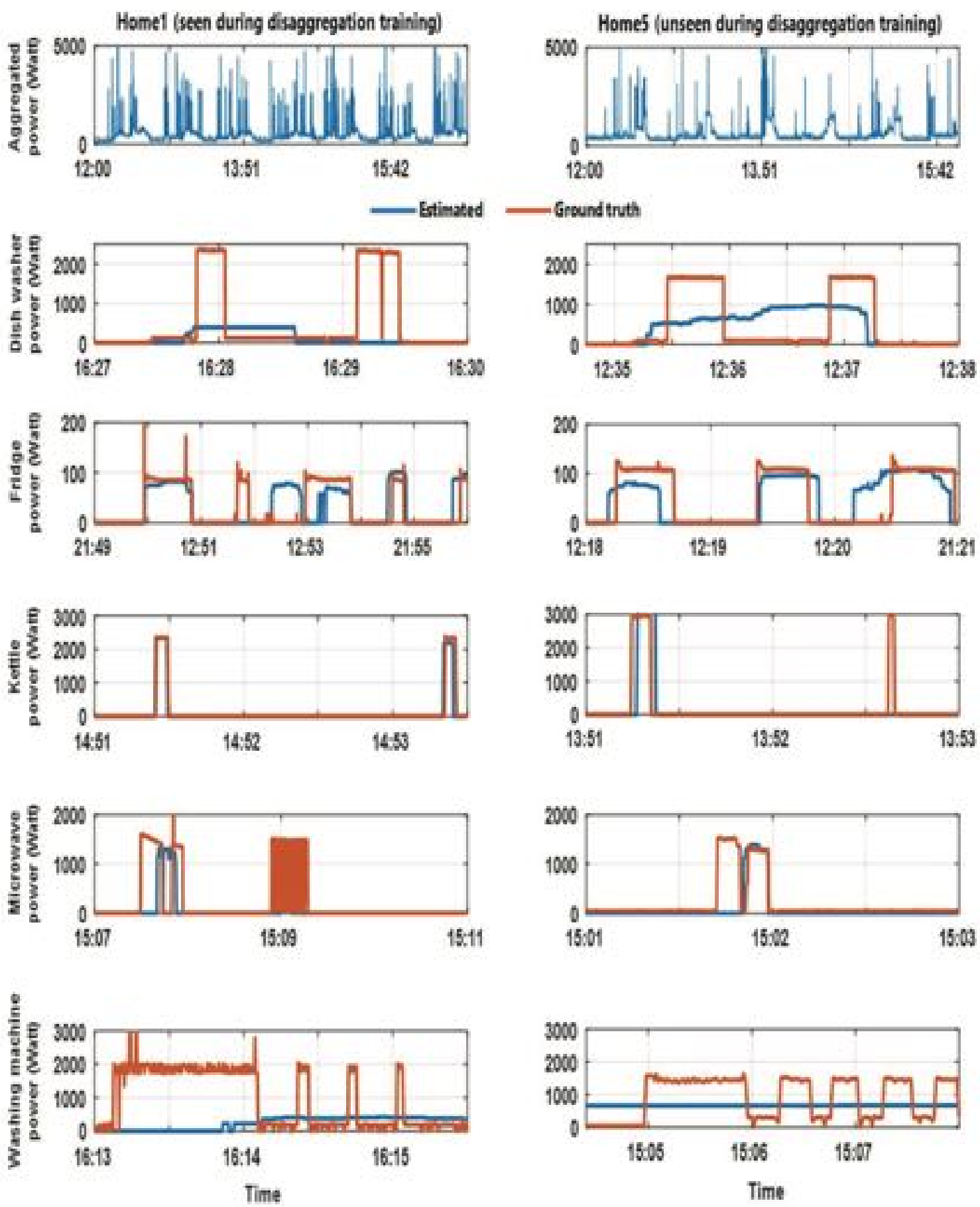

Figure 5.6 Shows the energy disaggregation algorithm output by using RNN or LSTM technique. (a) Aggregated power consumption for the home; (b) Dish Washer estimated and Ground truth power demand; (c) Fridge estimated and Ground truth power demand; (d) Kettle estimated and Ground truth power demand; (e) Microwave estimated and Ground truth power demand; (f) Washing machine estimated and Ground truth power demand 
disaggregation algorithm. The figure has six rows described as follows: (a) aggregated power consumption for the home; (b) comparison between the estimated and ground-truth power demand for the dishwasher; (c) comparison between the estimated and ground-truth power demand for the fridge; (d) comparison between the estimated and ground-truth power demand for the kettle; (e) comparison between the estimated and ground-truth power demand for the microwave; (f) comparison between the estimated and ground-truth power demand for the washing machine.

\subsection{Disaggregation stage analysis}

To identify the best disaggregation algorithm was used in the NILM stage. Seven common classification metrics represented through equation 7-23:

$$
\begin{aligned}
& \mathrm{TP}=\text { number of true positive } \\
& \mathrm{FP}=\text { number of false positive } \\
& \mathrm{FP}=\text { number of false negative } \\
& \mathrm{P}=\text { number of positive in the ground truth } \\
& \mathrm{N}=\text { number of negative in the ground truth } \\
& \text { recall }=\frac{T P}{T P+F N} \\
& \text { precision }=\frac{T P}{T P+F P} \\
& \mathrm{~F} 1=2 \times \frac{\text { prcision } x \text { recall }}{\text { precision }+ \text { recall }}
\end{aligned}
$$


accuracy $=\frac{T P+T N}{P+N}$

$\mathrm{E}=$ total actual energy

$\hat{\mathrm{E}}=$ total actual energy

$y_{t}^{(i)}=$ appliance $i$ actual power at time $\mathrm{t}$

$\hat{\mathrm{y}}_{t}^{(i)}=$ appliance $i$ estimated power at time $\mathrm{t}$

$\bar{y}_{t}=$ aggregated actual power at time $\mathrm{t}$

the relative error in total energy $=\frac{|\hat{\mathrm{E}}-E|}{\max (E, \hat{\mathrm{E}})}$

mean absolute error $=1 / T \quad \sum_{t=1}^{T}\left|\hat{y}_{t}-y_{t}\right|$

$$
\text { recall }=\frac{T P}{T P+F N}
$$

Figure. 5.7 shows the comparison between the disaggregation analyses for the home, which was seen during training. Figure.6.8 shows the comparison between the disaggregation analyses for the home that was unseen during training. The denoising autoencoder and RECTANGLES outperform LSTM, FHMM, and CO in most of the metrics throughout the five appliances. Figure 5.7 and Figure 5.8 are divided into five columns and seven rows. The five-column represent five appliances labeled from the left as follows: Dish Washer; Fridge; Kettle; Microwave; Washing machine. The seven rows labeled from upper to lower as follows: F1 score Equation 14; Precision score Equation 13; Recall score Equation 12; Accuracy score Equation 15; Relative error in total energy Equation 21; Proportion of total 
energy correctly assigned Equation 23; Mean absolute error Equation 22. Therefore, the result at position $(1,1)$ represents the $\mathrm{F} 1$ score for the Dishwasher with five different data mining energy disaggregation algorithms. These five algorithms were the legend at the footer of the figures.
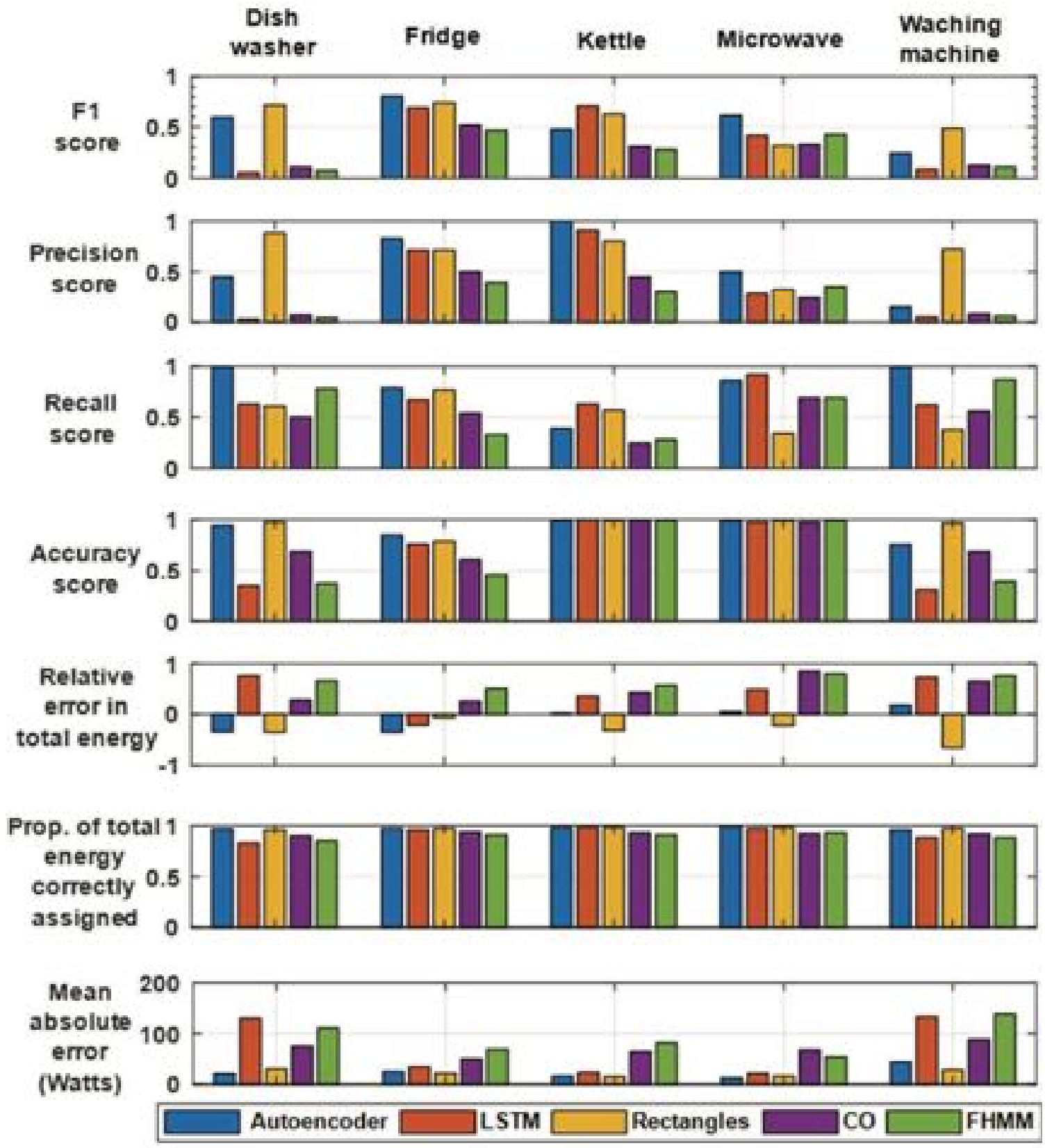

Figure 5.7 Energy disaggregation performance analysis for home seen during training 

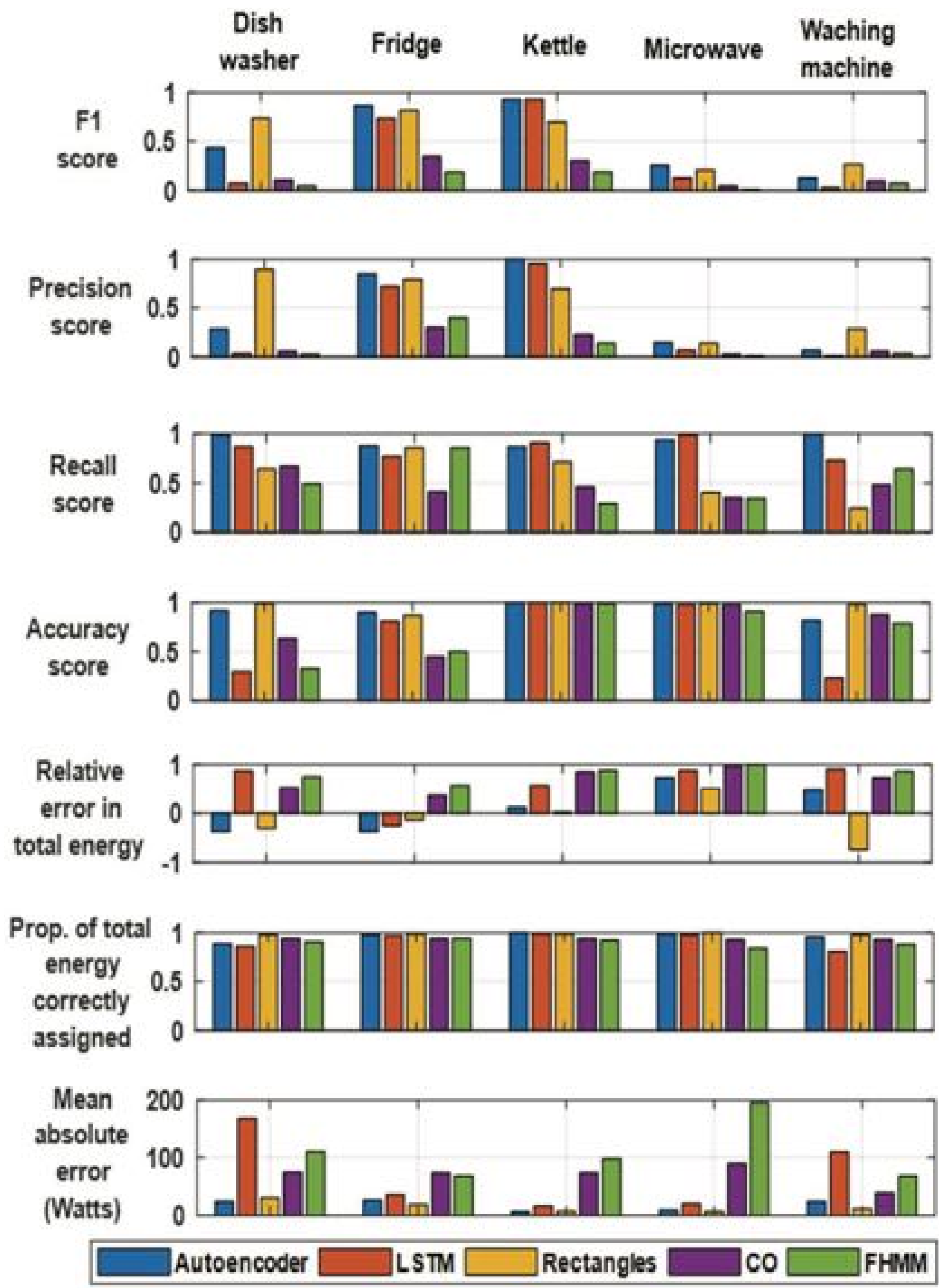

Figure 5.8 Energy disaggregation performance analysis for home unseen during training 


\subsection{The implemented Short-Term Load Forecasting}

A feed-forward Neural Network of the same design as used in [179] was employed, which is with eleven input nodes and one output node. One input for the current hour power

demand, one for an hour before, one for two hours before, one for a day before, one for the 23 hours before, one for 22 hours before, and five inputs for the five predicted appliances from the current power profile. The output node is for the expected hour ahead. In many ways, this test network presents a challenging forecasting case and these are all drawn from the real UK dataset.

\subsection{Simulation Results}

Figure 5.9 and Figure 5.10 demonstrate the actual load and the forecasted load by different methods for the home seen during the ED training and unseen during ED training, respectively. In order to assess the performance of the proposed method in conducting STLF for residential households, three widely used metrics were employed, including root mean squared error (RMSE), normalized root mean squared error, and mean absolute error. The three performance metrics are introduced in equations 24-26

$$
\begin{gathered}
R M S E=\sqrt{\frac{\sum_{t=1}^{N}\left(\hat{\mathrm{y}}_{t}-y_{t}\right)^{2}}{N}} \\
N R M S E=\frac{R M S E}{y_{\max }-y_{\min }} \\
M A E=\frac{\sum_{t=1}^{T}\left|\hat{y}_{t}-y_{t}\right|}{N}
\end{gathered}
$$




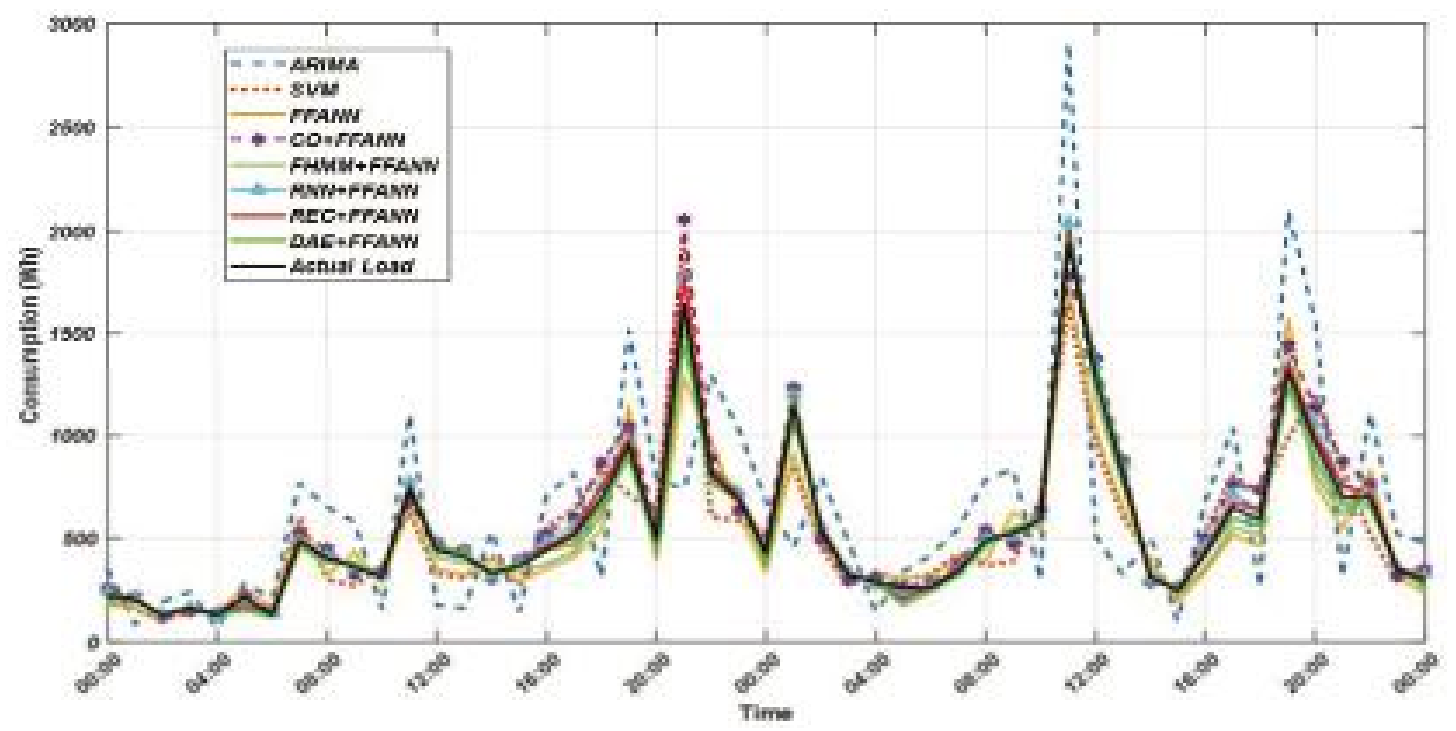

Figure 5.9 The actual and forecasted load by different methods for the home seen during the NILM training

Table 5.1 Load Forecasting Performance Comparison for the seen home

\begin{tabular}{llll}
\hline \hline Architecture & RMSE(kwh) & NRMSE & MAE(kwh) \\
ARIMA & 0.3831 & 0.1906 & 0.2935 \\
SVM & 0.1369 & 0.0749 & 0.1145 \\
FFANN & 0.1145 & 0.0627 & 0.0942 \\
CO + FFANN & 0.0877 & 0.0480 & 0.0641 \\
FHMM + FFANN & 0.0580 & 0.0318 & 0.0457 \\
RNN + FFANN & 0.0382 & 0.0209 & 0.2880 \\
DAE + FFANN & 0.0309 & 0.0169 & 0.0228 \\
\hline \hline
\end{tabular}




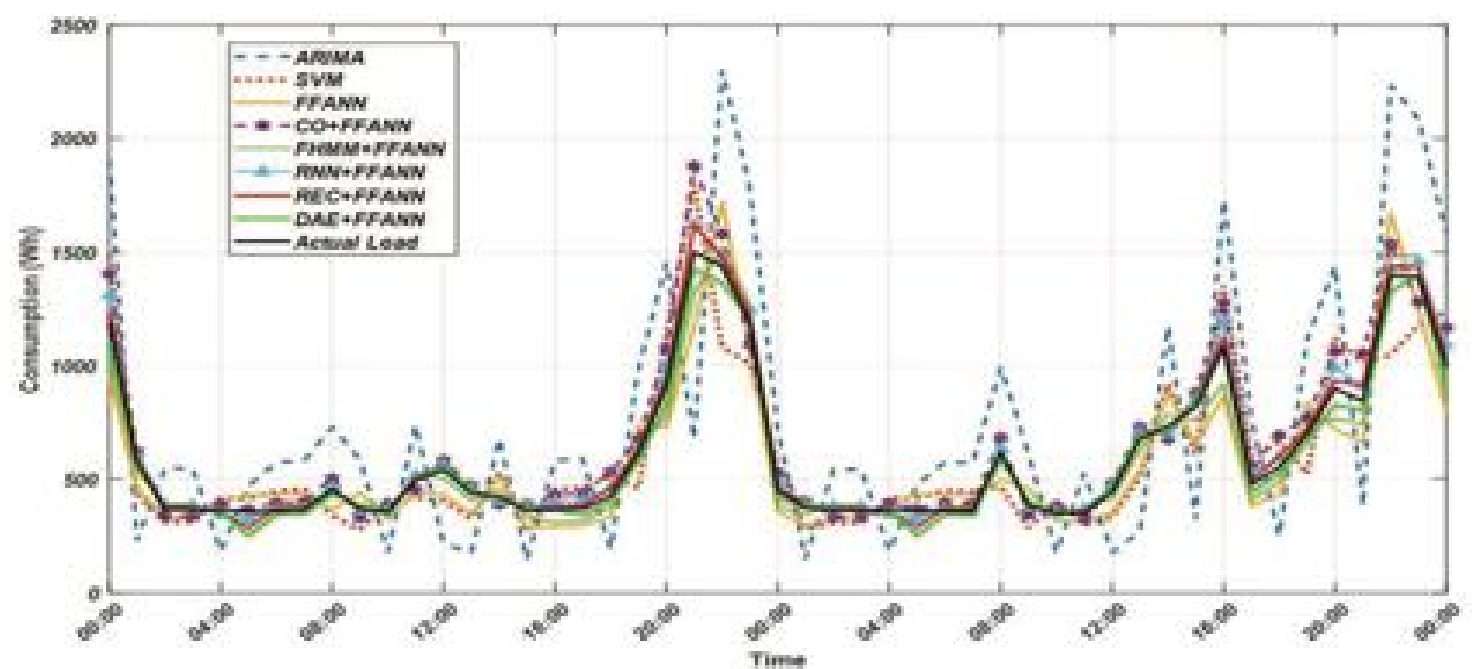

Figure 5.10 The actual and forecasted load by a different method for the home was unseen during the ED training

Table 5.2 Load Forecasting Performance Comparison for the unseen home

$\begin{array}{llll}\text { Architecture } & \text { RMSE(kwh) } & \text { NRMSE } & \text { MAE(kwh) } \\ \text { ARIMA } & 0.4280 & 0.1854 & 0.3506 \\ \text { SVM } & 0.1665 & 0.0722 & 0.1359 \\ \text { FFANN } & 0.1397 & 0.0605 & 0.1112 \\ \text { CO + FFANN } & 0.1145 & 0.0496 & 0.0770 \\ \text { FHMM + FFANN } & 0.0769 & 0.0333 & 0.0558 \\ \text { RNN + FFANN } & 0.0507 & 0.0219 & 0.0354 \\ \text { DAE + FFANN } & 0.0432 & 0.0187 & 0.0287 \\ \text { REC + FFANN } & 0.0372 & 0.0161 & 0.0268\end{array}$


Table 5.1 and Table 5.2 compare the performance of the proposed approach regarding RMSE, NRMSE, and MAE with the current state of the art techniques, i.e., AIRMA, SVM, and FFANN. As illustrated, the five proposed approaches; DAE + FFANN, REC + FFANN, RNN + FFANN, FHMM + FFANN, and CO + FFANN outperform FFANN, SVM, and ARIMA in all metrics used. In the case of Table 5.2 that should be the worst because, the data was unseen during the energy disaggregation stage. The proposed (REC

+ FFANN) brings a $91.13 \%$ reduction in RMSE and NRMSE, $92.36 \%$ reduction of MAE as compared with ARIMA.

\subsection{Conclusion}

This paper explored the potential of employing a pre-processing stage of NILM techniques combined with FFANN for household STLF under high uncertainty and volatility. As illustrated, the three proposed approaches; Rec + FFANN, RNN + FFANN, and DAE + FFANN, outperform FFANN, SVM, and ARIMA in all metrics used. The proposed deep learning techniques used for NILM stage were generalized even with data from unseen home. The best approach used for energy disaggregation is denoising autoencoder that directly affected the performance of the STLF at the residential household level. The proposed (DEA + FFANN) brings $91.13 \%$ reduction in RMSE and NRMSE, $92.36 \%$ reduction of MAE as compared to ARIMA. 


\section{Chapter 6 Power Quality Enhancement of Low Voltage Distribution Grid}

A massive evolution of the residential energy consumption sector has emerged lately. It added more convenience for the customer but with a more added burden on the grid. For instance, the integration of renewable distributed energy sources and the modern expansion usage of the essential DC electrical equipment may cause severe power quality problems. For example, the integration of rooftop photovoltaic (PV) may cause unbalance and voltage fluctuation, which can add constraints for further PV integrations to the network and the deployment of DC native loads with its nonlinear behavior increase the harmonics to the network. This work demonstrates a smart integration of a DC Microgrid to Neighborhood Low Voltage Distribution Network (NLVDN). The DC Microgrid connected to the NLVDN through a three-phase voltage source inverter (VSI). In which, the VSI works as Distribution Static Compensator (DSTATCOM), and the DC link provides an integration point for the PV and the DC native loads. The proposed technique is able to compensate for unbalance, reactive power and harmonics demanded by three-phase and non-linear loads and unbalanced connected to the distribution side, leading to the power quality enhancement. Besides, it can prevent the source from getting overloaded by providing active power support to the load.

\subsection{Introduction}

The predicted massive utilization of photovoltaic (PV) systems in the residential sector is justified for several reasons. The efficiency of the PVs is improving, the cost of 


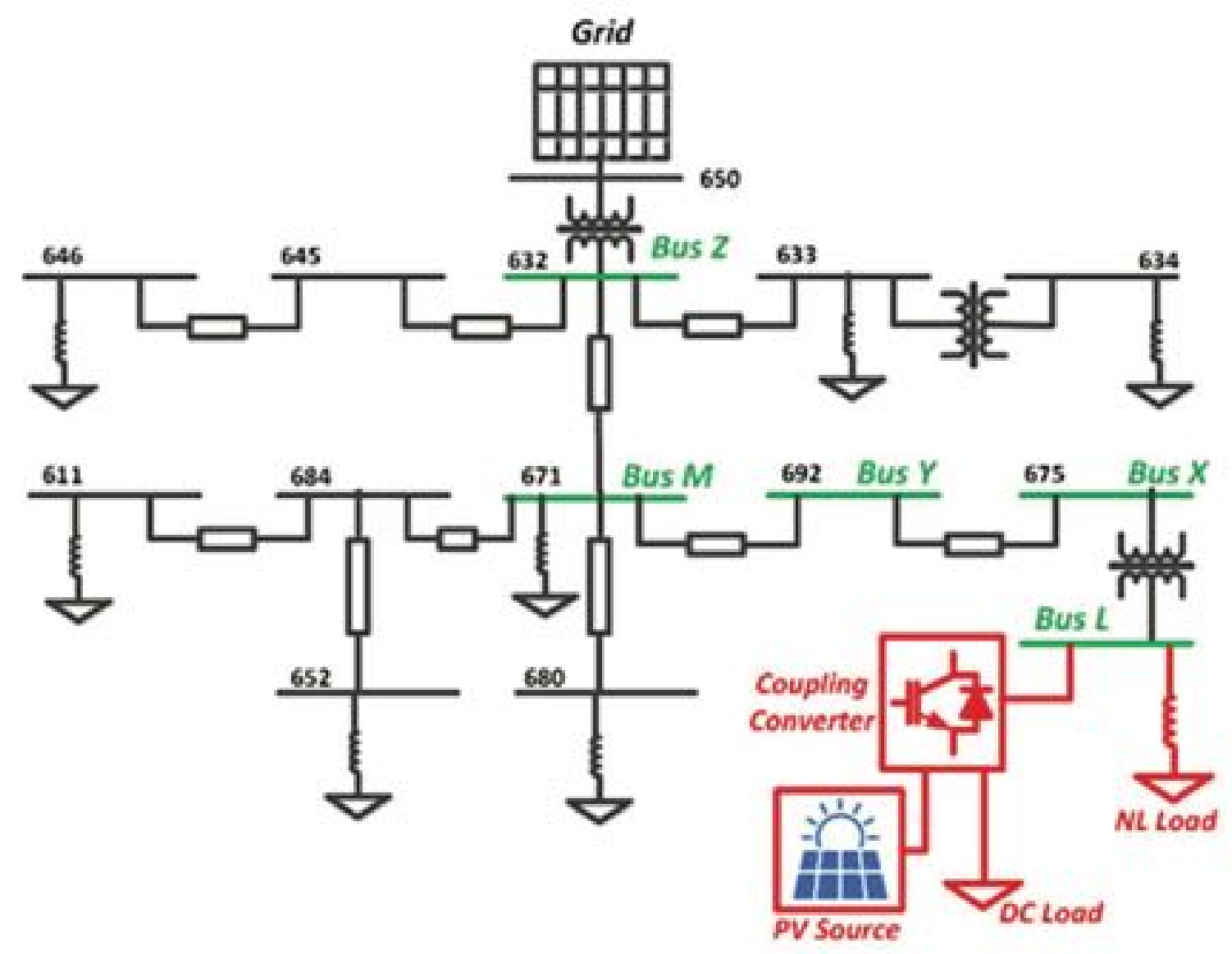

Figure 6.1 IEEE 13-bus test feeder system with a smart load

manufacturing is declining, and the incentives provided by the utility to decrease the utility bills are a fact. Also, it is expected that the DC loads usage is increasing in the distribution network. Such as the usage of new DC appliances and equipment in the residential sector (consumer electronics, LED lighting, and compact fluorescent lamps) [180]. Despite the benefits of the innovations at source and demand sectors, many power quality problems are facing the distribution network against all these innovations. For example, the integration of rooftop PVs may cause severe voltage fluctuations and unbalance due to the uncertainty and the lake of availability of the irradiance [181]-[184]. Also, the increased usage of DC Loads adds more harmonics to the distribution network [185], [186]. That is 
because all individual DC load needs an individual rectifier to facilitate the connection of the equipment to the current $\mathrm{AC}$ network and provide power factor correction to comply with the utility policy [187], [188].

Moreover, the expedite movement toward the usage of a sensitive component in residential, commercial, industrial, traction applications such as refrigerators, televisions, computers, switched-mode power supplies add nonlinearly, and unbalanced loads to the distribution network [189]. Degrading the power quality of the power system network wears out the network component, increases the probability of system shutdown, and increases the maintenance disbursal [190 - 192]. Consequently, enhancing the power quality of the electric power network is a critical factor in the process of power generation and delivery.

Consequently, this work proposes a smart integration of a DC Microgrid to Neighborhood Low Voltage Distribution Network (NLVDN), as depicted in Figure .6.1. The DC Microgrid connected to the NLVDN through a three-phase voltage source inverter (VSI). In which, the VSI works as Distribution Static Compensator (DSTATCOM), and the DC link provides an integration point for the PV and the DC native loads. The DC Microgrid composed of a Photovoltaic (PV) source and DC loads connected to the DC side, DC link capacitor bank, and three-phase VSI to integrate to the NLVDV.

The electrical regulation standards in distribution networks such as power factor and harmonics levels are applied to all AC loads connected to the current AC network. Some of these regulations define the interconnection requirement of the distributed generation (DG) units in low-voltage distribution systems [193]-[195]. Others determine the 
interconnection regulation for electric loads, such as home appliances [196]-[198]. The main concern for utility companies is the massive utilization of loads in current homes that do not comply with the standard regulation and degrade the power quality of the power system network [199].

The IEEE 1547 is one of the power quality standards, which define the harmonics and total harmonic distortion (THD) levels. Several works introduce different ways to mitigate harmonic components and compensate for reactive current, negative harmonics. Some use the injection of negative reactive current to the power network to reduce the harmonics and provide power factor correction [200]-[202]. Another approach allows the simultaneous compensation for the harmonics and reactive power issues called Active Power Filter (APF). Besides, it can provide compensation for the system load unbalance and compensate for voltage fluctuations at the point of common coupling (PCC). The different topology of APF, such as series and shunt. The performance of the APF is depended on the accuracy of selecting a suitable method to extract undesired current. The Synchronous Reference Frame (SRF) method is used in three-phase balanced systems [204]-[205]. Another effort work based on instantaneous power theory in three phases, the unbalanced system [206]-[209]. However, the previous approach requires extra computation to process the three phases current and the voltage measured, which affect the cost of implementation. In this paper, the smart integration of a DC Microgrid to Neighbourhood Low Voltage Distribution Network (NLVDN) was demonstrated. The DC Microgrid connected to the NLVDN through a three-phase voltage source inverter (VSI). In which, the VSI works as Distribution Static Compensator (DSTATCOM), and the DC link provides an integration 
point for the PV and the DC native loads. The proposed technique is capable of compensating reactive power, unbalance and harmonics demanded by three-phase nonlinear loads and unbalanced connected to the distribution side, improves the power quality. Besides, it can prevent the source from getting overloaded by providing active power support to the load.

The work is arranged as follows: In Section 2, a description of the system under study and the suggested control technique is demonstrated. In Section 3, simulation results are presented and inquired about validating the proposed technique. In section 4, experimental results are presented to evidence the validation of the controller's execution experimentally. Finally, in Section 5, the conclusions of the work contribution are listed.

\subsection{Proposed system description and the control technique}

\subsubsection{The proposed system description}

In order to evaluate the effect of a smart load on the distribution system, the smart load is connected to the IEEE 13-bus test feeder system. The test system is modified to include the smart load. Figure 6.1 shows the standard IEEE system under study, and the smart load is connected at bus 675 . The network bus $632,671,692$, and 675 are renamed to bus Z, M, $\mathrm{Y}$, and $\mathrm{X}$, respectively. The effect of imposing the smart load on the distribution system is studied on the pass from bus $\mathrm{Z}$ to bus L. The smart load contains a non-linear load, which is connected directly on bus L. The PV source and the DC load are connected to grid via a coupling converter. On the one hand, the converter works to harvest the PV energy and drive the DC load. On the other hand, the proposed control system provides ancillary 
service and ensures the power quality at the point of the common coupling, which positively impacts the distribution grid.

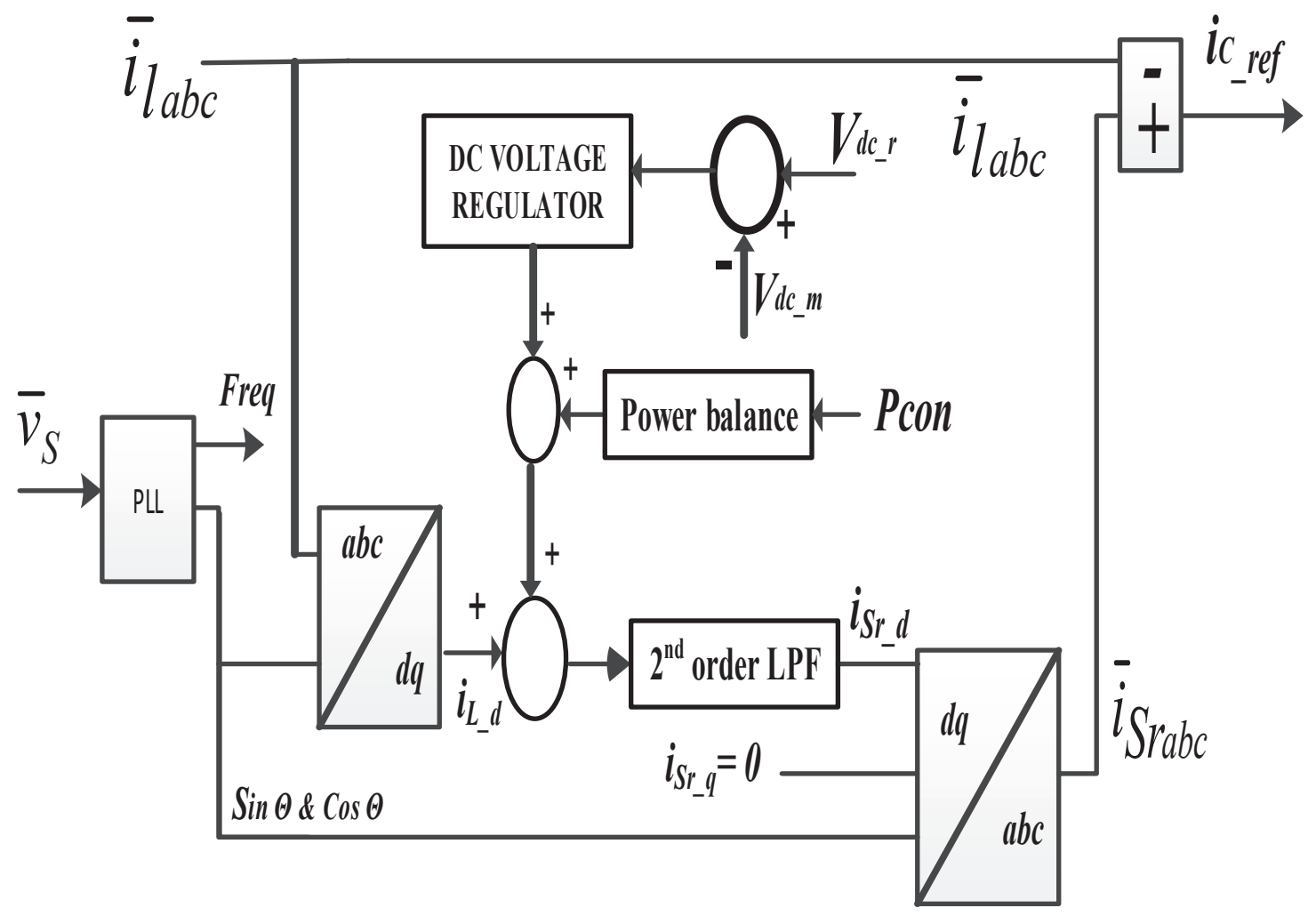

Figure 6.2 A block diagram for the proposed control technique

\subsubsection{The control technique}

The main functions of the control technique are canceling the harmonics caused by the non-linear loads, compensating for the load unbalance caused due to the existence of single and three-phase powered loads that are arbitrarily distributed, and act as power factor corrector (PFC) at PCC. The proposed algorithm is based on a modified version from vector-decoupled control. In which the instantaneous three phases current measured and transformed from $\mathrm{ABC}$ to $\mathrm{DQ}$ reference frame. This revised version of the vector decouples 
control technique capable of dealing with unbalanced and balanced systems. A block diagram depicting the control algorithm is shown in Figure 6.2.

This controller is based on the park's transformation. In which the three-phase current is converted to direct and quadrature current ild, and ilq respectively. This calculated current ild is analyzed. The analysis result gives a DC value representing the fundamental active component, and $\mathrm{AC}$ value representing the harmonic components. Unlike the vector decouple control method, which uses the high pass filter to isolate the AC component from ild. The proposed modified vector decouple control method uses a low-pass filter with a cut-off frequency of $75 \mathrm{~Hz}$. The DC component of ild is obtained by passing ild through the low pass filter. The low pass filter output represents the magnitude of the fundamental active current component existing in the load current. To regulate the DC bus voltage, a power balance technique used to control the power delivered and submitted to/from the DC link. By maintaining the power balance through the DC Microgrid, the amount of power controlled by the grid tie converter needs to be obtained, as given by equation (1), which represents the difference of power between the PV power and the local DC load power. The active power component controlled as given in equation (2). Since the synchronous reference frame $\mathrm{d}$-axis is aligned with the three-phase voltage angle, $\mathrm{V}_{\mathrm{q}}$ will be equal to zero. Then, the Id reference is calculated from equation (3).

$$
\begin{gathered}
\text { Pcon }=\text { Ppv }- \text { Pldc } \\
\text { Pcon }=\frac{3}{2}(V d * I d+V q * I q) \\
I d=\frac{2 P c o n}{3 V d}
\end{gathered}
$$


In which a PI controller will preserve this balance and provide a direct current component to represent the voltage regulator's direct current share. The previous component is added to the calculated ild (low pass filter output). Then, by using inverse Park's transform, the three-phase sinusoidal current reference is obtained. The quadrature current component ilq and zero current component il0 were set to zero since the target is to capture the active fundamental component only. Consequently, the obtained three-phase sinusoidal current reference is subtracted from the load current. The resultant reference current represents all harmonics and reactive components associated with the load current plus a fundamental component necessary for power balancing. The obtained three-phase sinusoidal reference is being subtracted from the load current to obtain the final current reference controls the injected current to the AC side. Therefore, the injected current will contain a component to balance the power flow and component to counteract the harmonics and unbalance values at the original non-linear load. Which mean pure current from the utility side at the PCC. Also, it maintains the load balance at bus L

\subsection{Results and Discussion}

\subsubsection{Case study A}

In this case study, the smart load control system is applied to a $\mathrm{t}=1.5 \mathrm{sec}$. Moreover, the following figures depict the comparison between the IEEE 13-bus system before and after the smart load application. To show the improved performance after applying the smart load, Figure 6.3 shows the effect of the smart load on active power, reactive power, and voltage profiles at bus $\mathrm{X}, \mathrm{M}$, and $\mathrm{Z}$. The results show that the active power capability for 
the whole pass of the smart load is increased. In addition, the reactive power at the same pass is reduced, which improves the power factor and the voltage profile at these points.
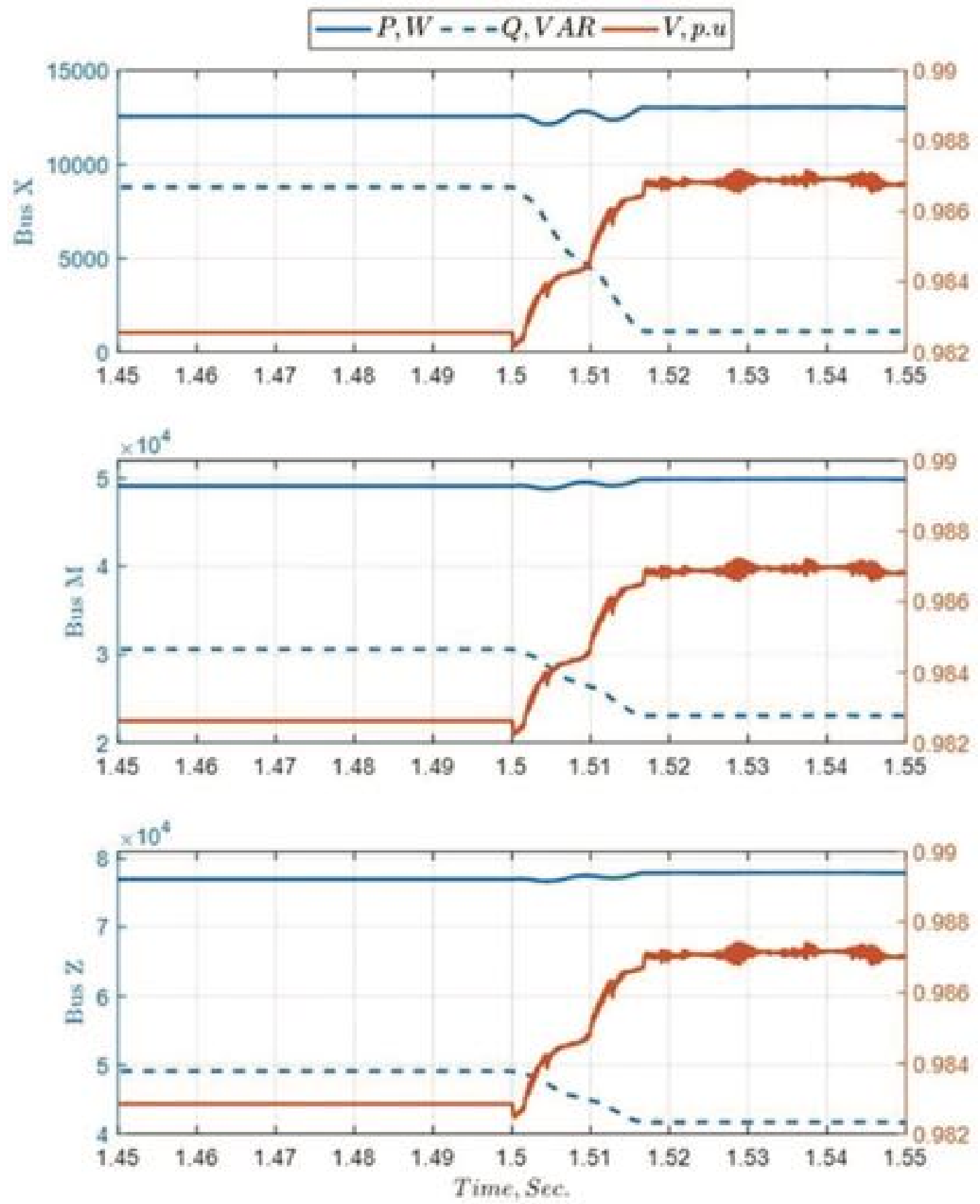

Figure 6.3 A comparison between the active, reactive power, and the PU voltage at buses X, $\mathrm{M}$, and $\mathrm{Z}$, respectively 

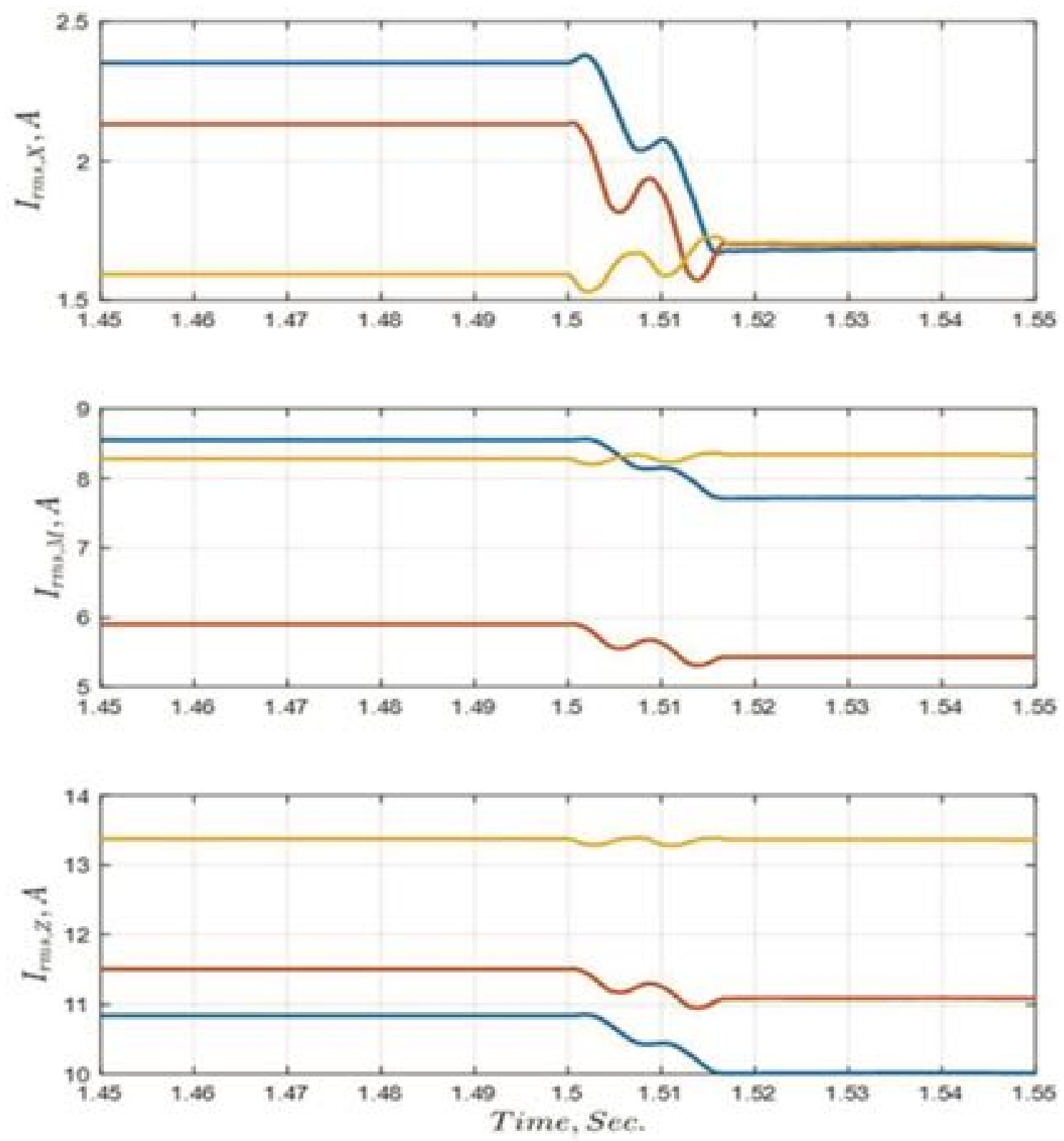

Figure 6.4 A comparison between three-phase current (RMS) at buses X, M, and Z, respectively, respectively

Correspondingly, as shown in Figure 6.4, the unbalanced current ratio is diminished through the distribution bus pass, especially at point X. To clearly show the effect of the smart load application, the instantaneous values of three-phase voltages, load currents, coupling converter currents, and bus L injected currents are illustrated in Figure 6.5. The 
figure demonstrates that the non-linear load and the converter compensation current to balance the bus L (PCC) three-phase current.
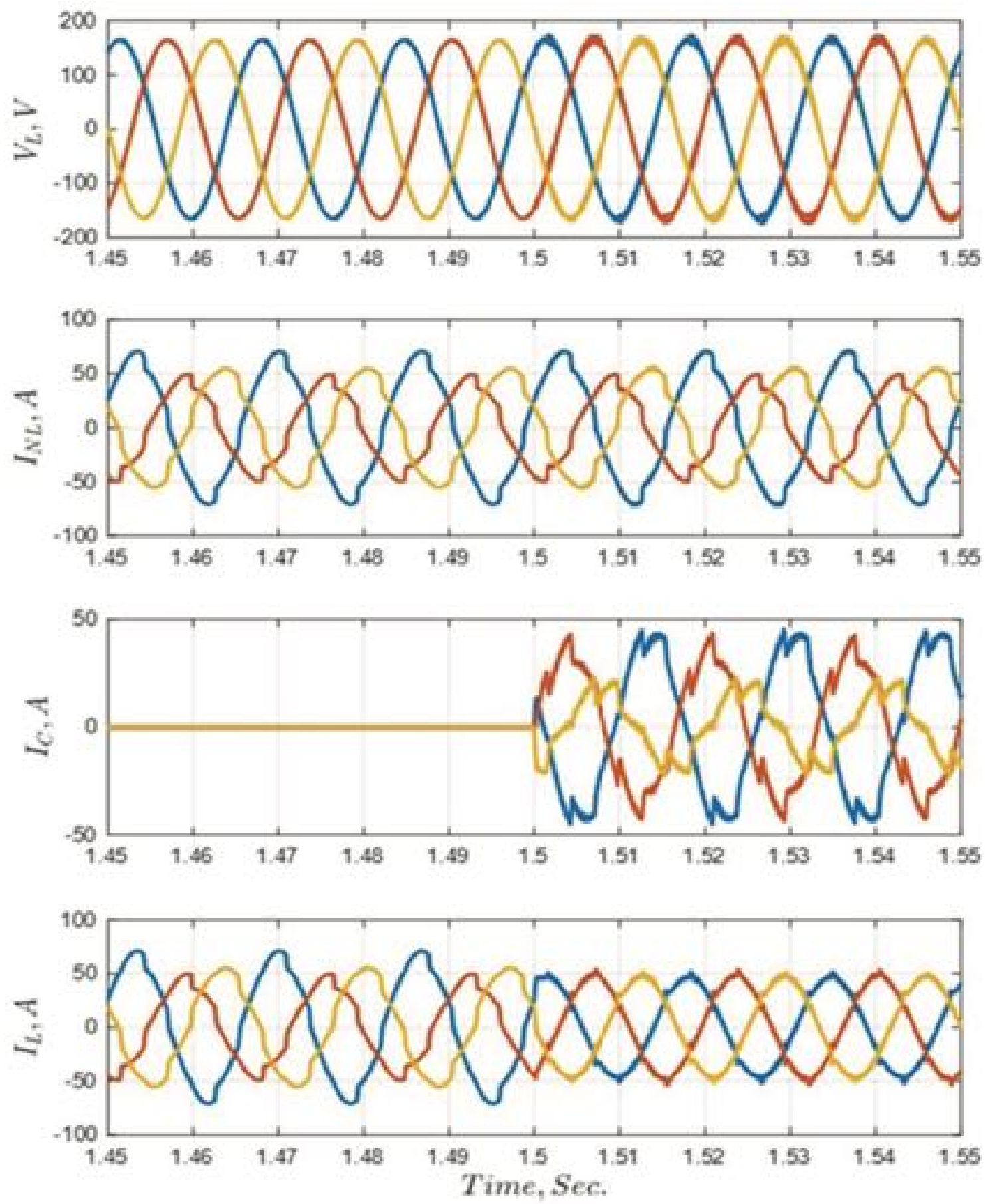

Figure 6.5 The three-phase voltage, load current, converter current, and bus current respectively at PCC 


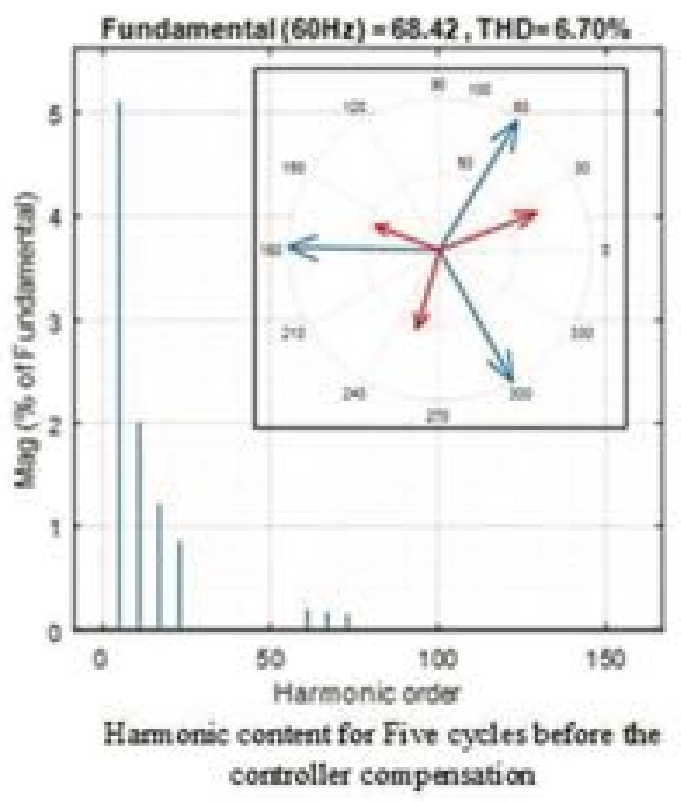

(a)

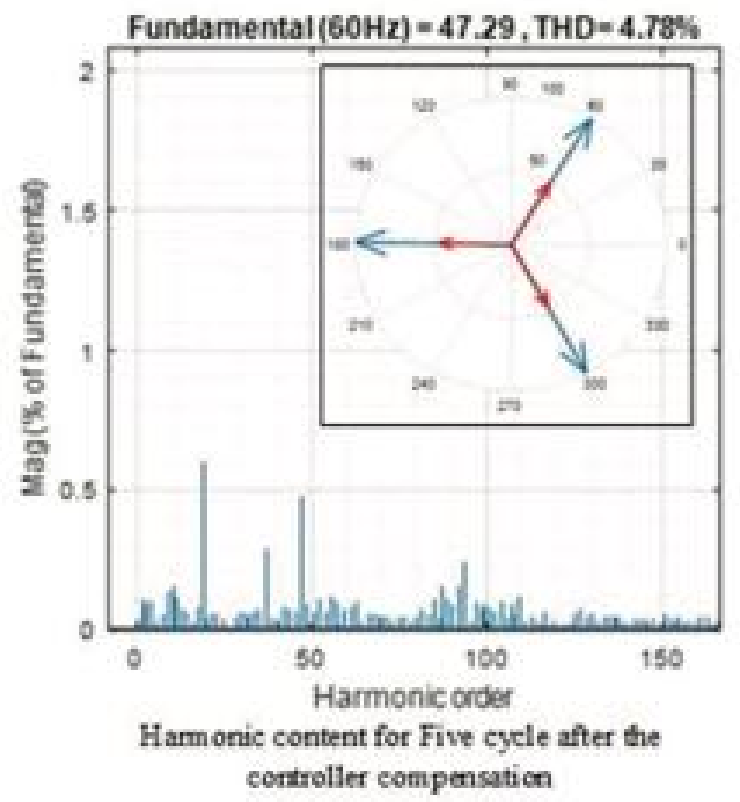

(b)

Figure 6.6 Phasor Diagram for the voltage and current at PCC, (a) before the proposed technique (b) after the proposed technique

Analytically, Figure 6.6 illustrates the harmonic analysis and vector diagram for five cycles before and after the controller compensation effect. In Figure 6.6 (a), the total harmonic distortion recorded $6.7 \%$. In addition, the current vector is not only unbalanced but also is shifted away from the voltage angle. On the other hand, Figure 6.6 (b) shows the effect of the smart load control system, the total harmonic distortion is reduced to $4.78 \%$ with balanced current, and the power factor is improved toward unity.

\subsubsection{Case study B}

In this case study, the controller will be examined to verify the capability for providing bi-directional power flow operation in addition to the power quality solving solutions. The simulation time is scaled to $24 \mathrm{sec}$ to represent a daily operation of 24 hours. 
(a) Bus Voltage

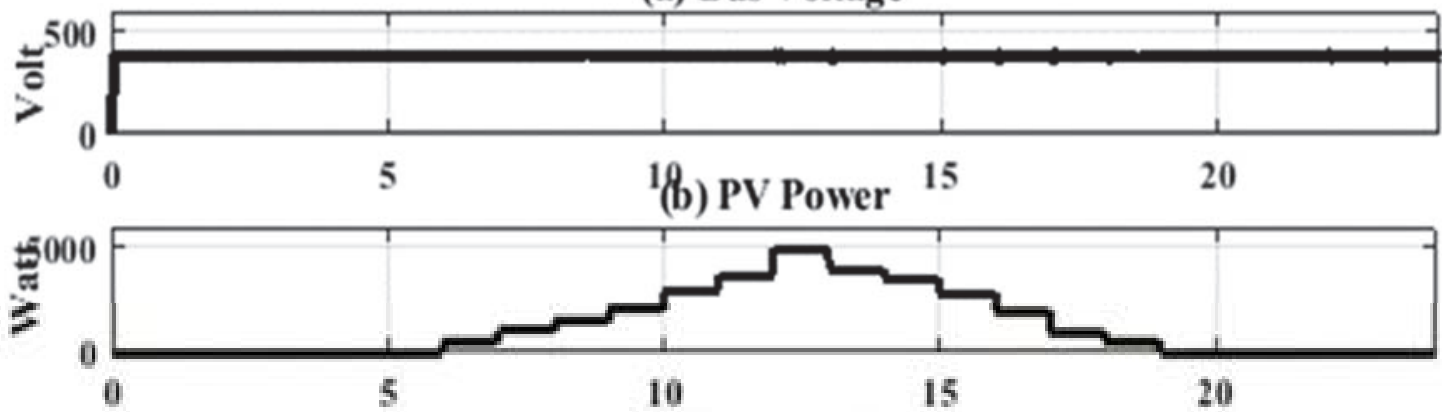

(c) DC Load Power

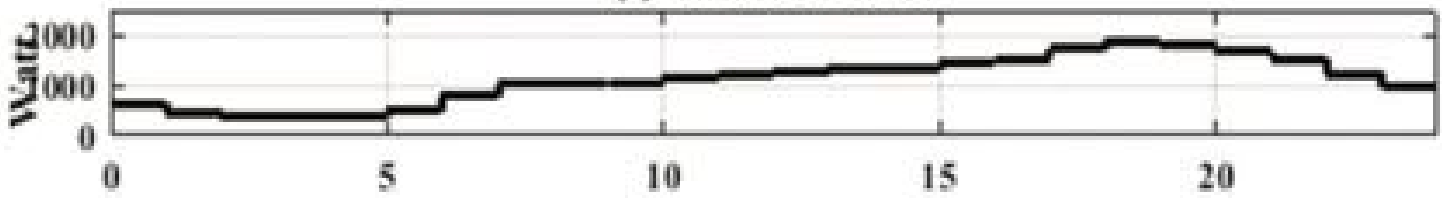

(d) AC/DC Converter Power

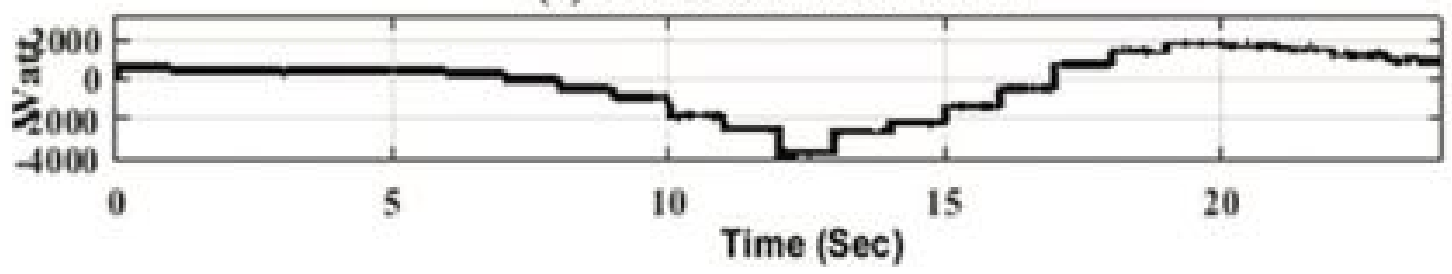

Figure 6.7 Simulation result: (a) DC link bus voltage (V), (b) output power of PV system (Watt), (c) Local DC load power (Watt), (d) The grid-tie converter power (Watt)

The controller examined under severe, bidirectional loading conditions to prove its capability to deal with such a load pattern. The DC voltage of the DC link bus is shown in Figure 6.7(a). It is noticed that the voltage is consistent at a constant value $(380 \mathrm{~V})$ during the whole running operation. Even at the load transition instant, the bus voltage ripple fluctuates between $370 \mathrm{~V}$ to $390 \mathrm{~V}$. this ripple does not exceed the $5 \%$ ratio allowed by the standards. The PV output power profile is shown in Figure 6.7 (b). The local DC load power of the Microgrid shown in Figure 6.7 (c). The power for the grid tie converter shown in Figure 6.7 (d). It can be noticed that the grid tie power between 0 to $7 \mathrm{sec}$ and 17 to 24 sec is positive, which indicates that the converter power flows from the $\mathrm{AC}$ grid to the $\mathrm{DC}$ 
microgrid (the converter is working in rectifier mode). The reason is: In these intervals, the PV power is not sufficient to feed the local DC load, the converter extracts power from the AC grid to supply the deficit in the DC Microgrid local load. However, between (7- 17 sec), the grid tie power is negative, which indicates that the converter power flows from DC microgrid to the AC grid (the converter is working in the inverter mode). The reason is: In these intervals, the PV output power is exceeding the local DC power, so the inverter

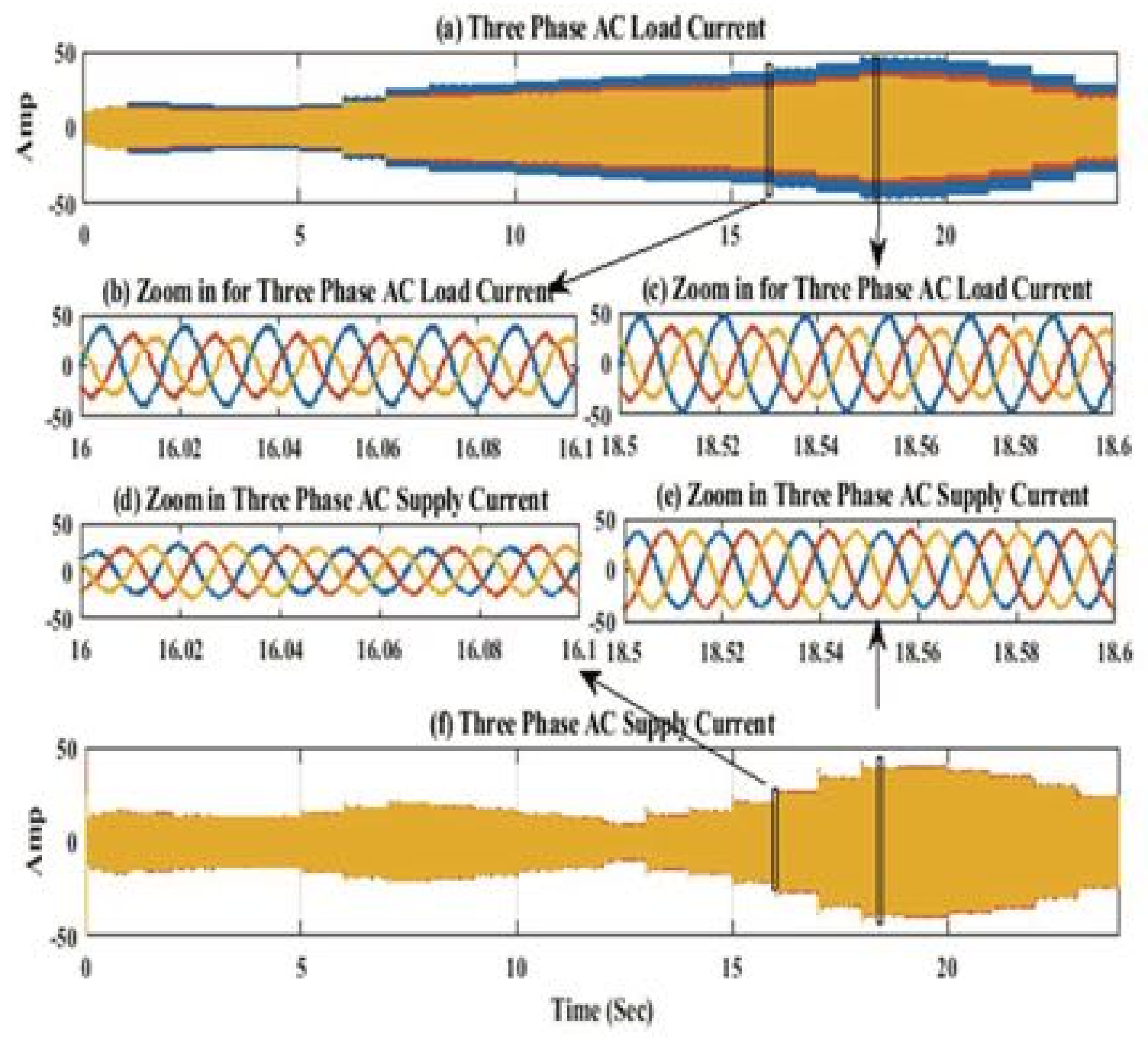

Figure 6.8 Simulation result: (a) AC load current, (b) Zoom in the AC load current (c) Zoom in for AC load current (d) Zoom in for AC supply current (e) Zoom in for AC supply current (f)

AC supply current 
extracts power from the DC Microgrid to support a local AC load on the AC utility side. Besides, the grid tie converter control proves its capability to control the power flow between both sides; it is also providing power quality solutions as illustrated in the description of the next figures

The three-phase AC load and source currents are shown in Figure 6.8(a) and Figure 6.8(f), respectively. Figure 6.8(a) shows the load current is an unbalanced, three-phase current, which is one of the problems that the controller has to tackle. Figure 6.8(b) and Figure 6.8(c) gives a close view of Figure 6.8(a) and shows the load current at two different intervals to demonstrate the performance of the system in both rectifier/inverter modes. It is noticed that the current waveforms suffer from noticeable distortion due to the existence of a nonlinear load. It can be seen in Figure 6.8(d) and Figure 6.8(e) that the three phases are balanced and the current waveforms are uniform. Which means the ability of the controller to mitigate harmonics and compensates for unbalances under both modes of operation

\subsection{Hardware Implementation \& Experimental Results}

To investigate the feasibility of the proposed control technique, a hardware setup has been established as depicted in Figure 6.9. The DC building connected at the PCC through $\mathrm{DC} / \mathrm{AC}$ converter. It has been implemented in the power system test-bed (Energy System Research Laboratory, Florida International University) [208]. It consists of AC and DC zones connected through an AC-DC converter. A DC bus represents the $\mathrm{DC}$ zone, where its voltage was set to $380 \mathrm{~V}$, a DC load emulator to represent load appliances connected to the DC side, and a PV emulator to represent a rooftop PV source. 


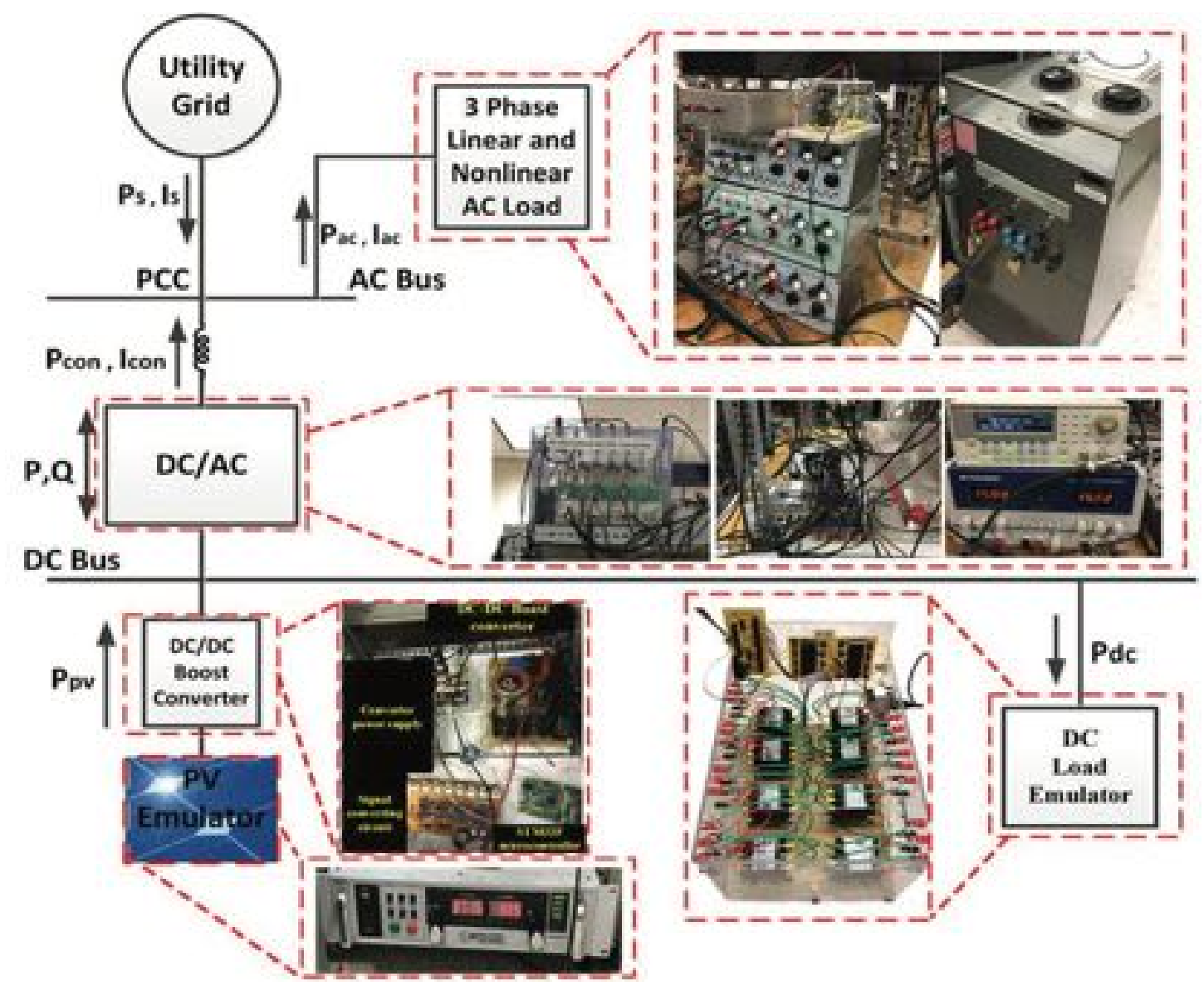

Figure 6.9 Experimental setup block diagram

It is interfaced with the DC bus through a DC-DC boost converter to extract maximum power generated by the PV system. The mathematical modeling of the PV system and the converter MPPT controller are built within the MATLAB/SIMULINK environment and executed with the DSpace 1104 real-time interface. The DC load emulator comprises of a combination of eight resistors with different values $(1 \Omega, 5 \Omega, 10 \Omega, 20 \Omega, 30 \Omega, 40 \Omega, 50$ $\Omega$, and $60 \Omega$ ) in a particular arrangement [210]. In addition to these load resistor combinations, eight controlled switches are used to change the connection topology. Which means, changing the values of the equivalent load resistors to obtain different load patterns. The central concept of operation is based on sending control signals to the switches to 
change their states (on/off). By changing their states, the equivalent load resistance is changing. The control commands are generated from a load profile generator developed in the LabVIEW environment. The control commands are transferred through the PCI 6025E card to a circuit. This circuit is based on TEXAS INSTRUMENT inverting buffer module sn7406n. This module contains six inverters with open collector output. The RMS values for the voltage in the AC zone is set to $208 \mathrm{~V}$. The AC bus is connected to the utility grid at PCC. Also, different load models were designed to represent the AC load pattern [211]. One of the passive loads built has a switching capacity of 10 levels parallel of resistive loads from $300-\mathrm{W}$ to $3-\mathrm{kW}$ power in steps of $300-\mathrm{W}$ at a nominal voltage that can be switched to emulate various load patterns.

The parameters of the main components of the hardware setup are given in Table 6.1. The experimental results are shown in Figure 6.8; they show the capability of the proposed algorithm to compensate for the unbalance and nonlinearity of the load current in different modes of operation. This figure is divided into four regions separated by the red-dashed line. These four regions represent three different modes of operation. In mode one, (interval between $0-28 \mathrm{sec}$ ), the converter is working as an APF for the AC load but without any exchange in power between $\mathrm{AC}$ and $\mathrm{DC}$ zones.

In mode two, (interval between $28-88 \mathrm{sec}$ and $188-278 \mathrm{sec}$ ), the converter works as an $\mathrm{APF}$ and rectifier in which the power is transferred from the AC side to the DC side. In mode three, (interval between $88-188 \mathrm{sec}$ ), the converter works as an APF and inverter in which the power is transferred from the DC side to the AC side. 
Table 6.1 the experimental setup Parameters

\begin{tabular}{ccc}
\hline Component & Parameter & Specification \\
\hline & power rating & $2500 \mathrm{~W}$ \\
Boost Converter & IGBT module & SKM100GAL12T4 \\
& switching frequency & $5 \mathrm{kHz}$ \\
& LBC, RLBC & $6 \mathrm{mH}, 0.21 \Omega$ \\
\hline \multirow{2}{*}{ Bidirectional } & power rating & $1800 \mathrm{~W}$ \\
AC/DC Converter & IGBT module & SK45GB063 \\
& switching frequency & $10.89 \mathrm{kHz}$ \\
\hline AC Filter & L AF, RLAF & $12 \mathrm{mH}, 0.31 \Omega$ \\
\hline
\end{tabular}

The DC voltage of the main DC bus is shown in Figure 6.10(a). It is noticed that the voltage is stable during the whole operation at $380 \mathrm{~V}$. The ripple in the voltage fluctuates in an acceptable range between $370 \mathrm{~V}$ to $390 \mathrm{~V}$ at the instant of load transition. This small fluctuation in the DC bus voltage is compatible with the standards, as it does not exceed 5\%. The grid tie converter power is shown in Figure 6.10(b). Which explains the bidirectional power flow between the $\mathrm{AC}$ grid and the $\mathrm{DC}$ microgrid. It can be noticed that the grid tie power between 30 to $100 \mathrm{sec}$ and 188 to $278 \mathrm{sec}$ is positive, which indicates that the converter power flows from the $\mathrm{AC}$ grid to the $\mathrm{DC}$ microgrid (the converter is working in rectifier mode). However, between (100-188 sec), the grid tie power is negative, which indicates that the converter power flows from DC Microgrid to the AC grid (the converter is working in the inverter mode).

In response, the converter control verified its capability to control the bidirectional power flow between the DC building and the AC grid. The three-phase AC load and source currents are shown in Fig. 6.10(c) and Fig. 6.10(j), respectively. Fig. 6.10(d), Fig. 6.10(e) 
and Fig. 6.10(f) give a close view of Fig. 6.10(c) at modes one, two, and three, respectively. There is noticeable distortion in the current waveforms to the existence of nonlinear loads.

Fig. 6.10(g), Fig. 6.10(h) and Fig. 6.10(i) gives a close view of Fig. 6.10(j) at modes one, two, and three, respectively. This shows the controller's capability to mitigate the harmonics and compensate for unbalances under all modes of operation.

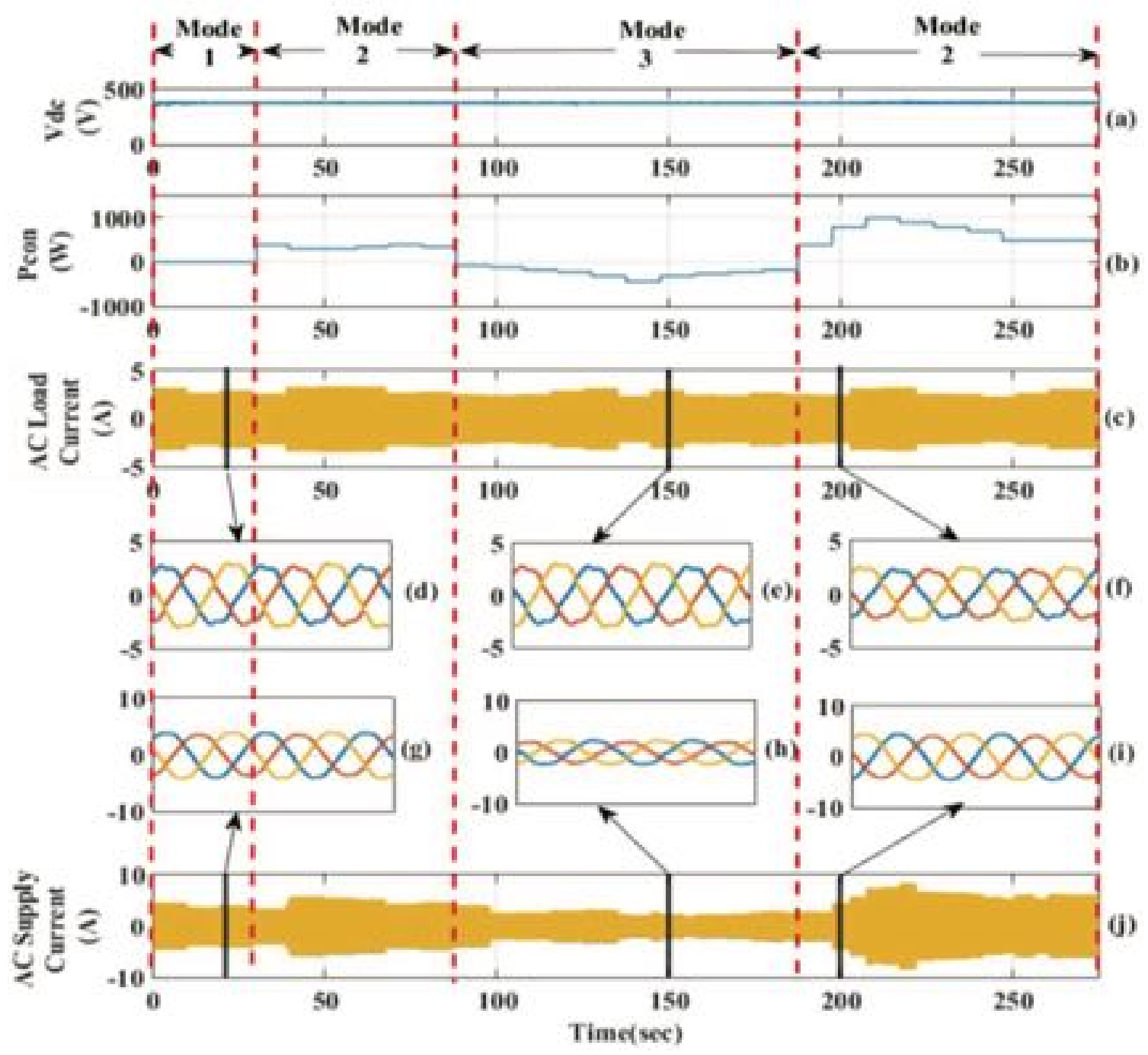

Figure 6.10 (a) DC link bus voltage (V), (b) the output power of PV system (Watt), (c) AC load current, (d) Zoom in for AC load current (e) Zoom in for AC load current (f) Zoom in for AC load current (g) Zoom in for AC supply current (h) Zoom in for AC supply current (i) Zoom in for AC supply current (j) AC supply current 


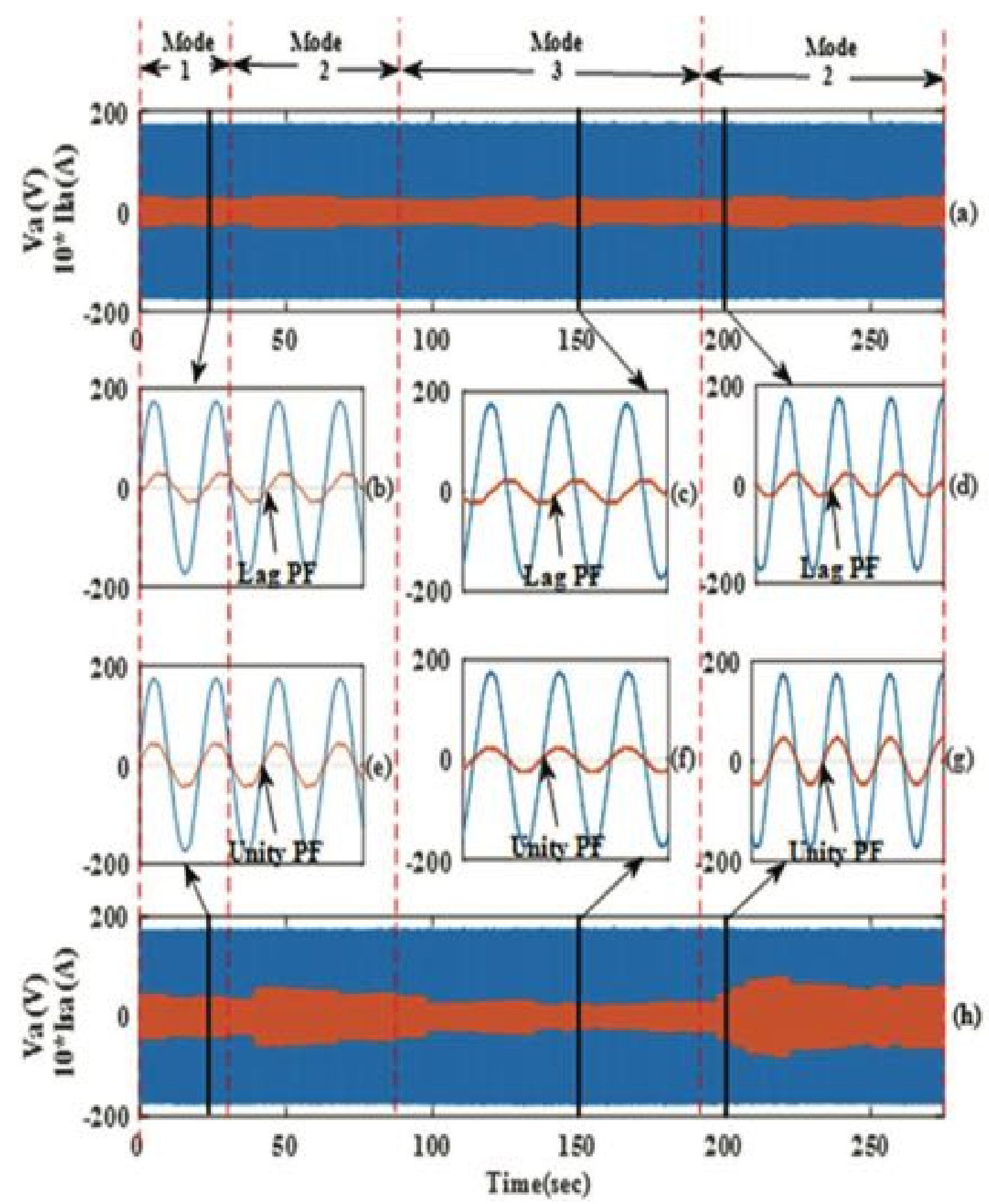

Figure 6.11 (a) Phase a Voltage (V) and load current, (b) Zoom in between (25.03-25.1) sec, (c) Zoom in between (150.03-150.1) sec, (d) Zoom in between (200.03-200.1) sec, (e) Zoom in for between (25.03-25.1) sec, (f) Zoom in between (150.03-150.1) sec, (g) Zoom in between (200.03-200.1) (h) Phase a Voltage (V) and phase a supply current. 
As is seen in Fig. 6.10(g), Fig. 6.10(h) and Fig. 6.10(i), the three-phase currents at the source side are balanced. This indicates that the grid tie converter's controller succeeds to cancel out power quality issues (distortion and imbalance) from the source side. In order to validate the controller capability to improve the power factor, Fig. 6.11 is shown. Fig. 6.11(a) shows the phase a's voltage versus phase a's the load current. Fig. 6.11(h) shows the phase a voltage versus phase a supply current. Fig. 6.11(b), Fig. 6.11(c) and Fig. 6.11(d) gives a close view of Fig. 6.11(a) at modes one, two, and three, respectively. It is noticed that the current waveform is out of phase and lagging the voltage waveform, which means lag PF. Fig. 6.11(e), Fig. 6.11(f) and Fig. 6.11(g) gives a close view of Fig. 6.11(h) at modes one, two, and three, respectively. It can be seen that the current waveform is in phase with the voltage waveform, which means unity PF. Thus, the proposed control algorithm id succeeds to correct the power factor for the system.

\subsection{Conclusion}

In this paper, a smart control technique for DC microgrid's grid-tie converter is proposed. It offers a solution for the near-future distribution network in a smart grid. It offers a power quality enhancement to the distribution network in the neighborhood. The controller is based on a modified vector decoupling control. This control technique enables the microgrid's grid-tie converter to act as DSTATCOM to maintain the power quality at the bus of connection in the best condition. By counteracting the unbalance and harmonics problems with the help of the smart integration of microgrid.

Moreover, the controller provides harmonic mitigation and power factor correction at the PCC. The controller is verified in MATLAB/SIMULINK for simulation purposes. The 
developed controller is then tested experimentally. Different loading conditions are simulated to prove the effectiveness of the system. Based on the simulation and the experimental results, it can be deduced that the compensation for reactive power and harmonics has been achieved effectively. The source current is balanced, sinusoidal, distortion-free and with improved power factor. The \%THD has reduced significantly after compensation. 


\section{Chapter 7 An Optimal Energy Management System for Real-Time Operation of Multiagent-Based Microgrids}

The real-time operation of the energy management system (RT-EMS) is one of the vital functions of Microgrids (MG). In this context, the reliability and smooth operation should be maintained in real-time regardless of load and generation variations and without losing the optimum operation cost. This work presents a design and implementation of an RTEMS based on Multiagent system (MAS) and the fast converging T-Cell algorithm to minimize the MG operational cost and maximize the real-time response in grid-connected MG. The RT-EMS has the main function to ensure the energy dispatch between the distributed generation (DG) units that consist of this work on a wind generator, solar energy, energy storage units, controllable loads, and the main grid. A modular multi-agent platform is proposed to implement the RT-EMS. The MAS has features such as peer-topeer communication capability, a fault-tolerance structure, and high flexibility, which make it convenient for the MG context. Each component of the MG has its managing agent. While the MG optimizer (MGO) is the agent responsible for running the optimization and ensuring the seamless operation of the MG in real-time, the MG supervisor (MGS) is the agent that intercepts sudden high load variations and computes the new optimum operating point. In addition, the proposed RT-EMS develops integration of the MAS platform with the Data Distribution Service (DDS) as a middleware to communicate with the physical units. In this work, the proposed algorithm minimizes the cost function of the MG as well as maximizes the use of renewable energy generation; then, it assigns the power reference 
to each DG of the MG. The total time delay of the optimization and the communication between the EMS components were reduced. To verify the performance of our proposed system, an experimental validation in an MG testbed was conducted. Results show the reliability and effectiveness of the proposed multiagent based RT-EMS. Various scenarios were tested, such as regular operation as well as sudden load variation. The optimum values were obtained faster in terms of computation time as compared to existing techniques. The latency from the proposed system was $43 \%$ faster than other heuristic or deterministic methods in the literature. This significant improvement makes this proposed system more competitive for RT applications.

\subsection{Introduction}

Technology has shaped our modern life in many aspects: starting with smart TV, passing by smartphones, and ending by Smart Grid. The power system, which is conventionally structured as a generation, transmission, and distribution, is reshaped as an agglomeration of Microgrids (MGs). The idea behind such a new structure is the increasing distributed energy resource (DER) mainly renewable energy that has been installed in the low voltage (LV) networks. Injecting power into the distribution power system is a new phenomenon that must be dealt with. This is where smart cyber systems interfere. Information technologies have been integrated with the power system enabling, consequently, the smart control of DG and make it possible for the MG to operable autonomously without being connected to the grid. Besides, such a structure favorites the interaction of the customer to become an active player in the system and control its load depending on the distribution network operator. 
The information system responsible for the control and economic optimization of MG is known as the energy management system (EMS). The EMS comprises three parts. The primary, secondary, and tertiary control. More details on the primary, the secondary, could be found in $[212,213]$. This work focuses on tertiary control. One of the most crucial tasks of a real-time EMS in grid-connected MG is to satisfy the minimum total operation cost while meeting the energy balance between different DER, storage units, controllable loads, and the power exchanged with the primary grid under their specific constraints. This is called in the literature review as the economic dispatch problem or the economic load dispatch problem. To solve this issue, several optimization methods have been used in the past decades. All of them can be categorized into two main categories: Classical and evolutionary algorithms.

In the conventional power system, the problem is modeled as a quadratic cost function, which is solved by classical methods such as the Lagrange multiplier method, base point participation factor, lambda iteration, Newton's method, gradient method, mixed-integer linear programming, and linear programming [214]. Most of the previous works presented a power dispatch of thermal generators, which makes the cost function considered not suitable for MG optimization [215, 216]. Besides, some of those used technics suffer from some limitations such as the assumption that the incremental cost curves of the generation units are monotonically increasing piecewise linear functions and the high dependency on the specific mathematical model. Even if dynamic programming could be used to solve the economic dispatch, it presents a complex solution that is not appropriate for real-time 
applications [217]. Furthermore, it is not possible to apply such methods in real-time, specially with an MG containing a large number of components.

In this respect, evolutionary algorithms were introduced to overcome those limitations. The heuristic evolutionary algorithms are inspired by the natural and social behavior of animals and organisms [214]. For instance, genetic algorithm, improved genetic algorithm was based on genes behavior, while evolutionary programming, ant colony metaheuristic, artificial bee colony, particle swarm optimization, and artificial neural network are based on swarm behavior of insects and micro-organisms. However, even if some of these methods present competitive results, still some of them suffer from the curse of local optimality in some cases [218]. The literature review reveals that most of the previous work was considering a day ahead optimization and not considering the real-time constraint [219]. More recent works present the real-time operation of energy management in MG [220, 221]. However, some of the work was based on simulations and did not experiment with the RT operation in the real testbed, while other work did not consider the point of a single failure of the system. An RT framework for EMS of MG using MAS was considered in [222], however, there was no cost optimization in the system.

Since deterministic optimization methods are time-consuming to reach the optimal solution for problems with large dimensions, evolutionary algorithms were introduced to overcome this limitation. The main advantage of these solutions is that they solve complex problems with a non-deterministic polynomial problem. Furthermore, in the MG context, while the EMS is running in real-time, solving the ED optimization time is also a crucial parameter. Thus, looking for a fast optimization technique is essential. 
In this work, the T-Cell algorithm is presented as a heuristic method inspired by immunobiology to solve ED in RT. The algorithm was first introduced in [223]. The TCell algorithm is inspired by the mediated immune cells in the human body. The T-Cell or as known in the immunobiology, helper T-cells play a key role in the adaptative immune response. During their life, helper T-cells experience three phases. During the first phase of their life, they develop in the Thymus, then they immigrate and start circulating between the bloodstream and lymphoid tissue. At this stage, they are known as a naïve helper Tcells. Then in the second phase, the naïve T-cells encounter an antigen-presenting cell. This latter binds to the peptide and protein receptors at the cell surface, which results in the TCell activation. Once activated, the cell starts proliferating and differentiating. This is the third phase. Helper T-Cell differentiate into either T helper 1 or $\mathrm{T}$ helper 2 effector cells, depending on their environment's composition. Each type of effector cell helps to eliminate the antigen presented in the activation process [224]. Therefore, during an antibody intrusion, the T-Cells proliferate by generating other clones of themselves; then, each clone differentiates by acquiring new proprieties to destroy the intrusion [225]. The authors in [226] tested the performance of the T-Cell algorithm compared to other optimization heuristics. The algorithm presents a competitive result in terms of convergence and execution time.

However, the optimization is just one part of an EMS. The EMS in the context of MGs deals with all management aspects of MG. It must coordinate between different DERs, supervise the network in case of load or generation fluctuation and make sure that energy balance is satisfied all time. A multi-agent system (MAS) is well suited in this context. 
MAS knew many improvements during the last years. The nature of the modular and decentralized structure increases researchers' interest to apply them in a power system context [227]. An agent is nothing but a cyber or cyber-physical entity that can act autonomously according to its environment, make decisions, and achieve its goals and interacts with its peers [228].

The distributed nature of MAS makes it suitable for the MG environment. Furthermore, their flexibility, extensibility, and fault tolerance have pertinently justified their adoption. Flexibility refers to the agent's ability to choose the most appropriate action; extensibility means the system can be extended easily; fault tolerance refers to the inherent redundancy mechanism build in MAS [229]. The central management of MG has been criticized for a long time by its drawback, the single point of failure. However, using a multiagent platform that implements fault tolerance capabilities would resolve this issue. For these reasons, MAS was adopted in many areas of the power system. The classic bidding mechanism of electricity, for instance, used the MAS as an efficient negotiation tool [230-233]. Finally, MAS was also implemented as a fundamental MG and smart building management platform [221, $234-237]$.

For these reasons, this work harvests the advantage of using a heuristic algorithm that is inspired by the immune system to optimize the ED and reaches the optimum in less time, even in complex systems with large dimensions. Besides, the use of MAS makes the system overcome the drawback of a single point of failure and adds fault tolerance feature in addition to the ability of easy extensibility in case of future system growth. 


\subsubsection{Target}

The present work's target is to implement the T-Cell algorithm as optimization methods for DED within MG based on agents' paradigm, and interoperability capabilities. The main contributions of this work are as follows:

- The implementation of an enhanced version of the T-Cell algorithm for RTEMS within MG;

- The integration of the Multiagent EMS with the testbed lab using DDS;

- Fault tolerance implementation of the multiagent EMS;

- The experimental validation and implementation of this architecture into a real $\mathrm{MG}$

- Competitive time delay optimization for real-time application.

This work presents a new optimization algorithm in the MG context. Although some heuristic optimizations were used in the EMS in MG, other system's features were not taken into consideration, such as the central aspect of the EMS or the failure case management. Excluding the fault, the case scenario makes the system vulnerable and suffers from a single point of failure. Moreover, the author's objective is to design a comprehensive system that tackles several aspects of the MG EMS: Optimization and communication as a cyber-physical system.

\subsubsection{System Component}

The present work uses different components to implement the proposed system. The MAS was adopted for the EMS within the MG. The multiagent platform comprises three main agent's categories. Management agent, backup agent, and interoperability agents. The 
management agent comprises the MG optimizer (MGO) and the MG supervisor (MGS). The backup agent is the MGO backup agent. The communication agents correspond to $\mathrm{MG}$ unit agents that manage communication between the RT-EMS and the real components of the MG. The presentation of each agent will be explained in section four with more details.

The Data Distribution Service (DDS) middleware was also proposed as an interoperability solution between the agent part, and the hardware part is assured by. The DDS is a middleware protocol and standard for data-centric communication for distributed real-time applications. It facilitates the development and integration of a distributed system and data exchange. The RT-EMS integrates the DDS components to communicate the computed references to the hardware part.

\subsubsection{Method}

The optimization of the DED in this work is solved by using the T-Cell heuristic algorithm inspired by the body's immune system. This algorithm mimics the human body defense mechanism. The heuristic technics based on biological process has several advantages compared to classical deterministic methods. One of the main advantages of these solutions is that they solve complex problems with a non-deterministic polynomial problem. The execution time of the optimization is improved, and the same time, an optimal solution is reached. These reasons are behind the motivation of using the T-Cell as techniques to solve our DED. Besides, the comparison of our algorithm to other existing ones shows better results in terms of optimum computation and time delay which makes it competitive for RT applications. 
The next section will cover the problem statement that includes the mathematical implementation of dynamic economic dispatch (DED). Section three describes the T-Cell algorithm that is implemented in the energy management system (EMS). Section four of this paper discusses the proposed multiagent platform that was designed in this study. Lastly, sections five and six present and address the findings and concluding remarks on applicability, reliability, and time delay.

\subsection{Problem Formulation}

As mentioned before, the EMS is the information system responsible for the economic optimization and the steady operation of the MG. Its primary function at a single MG is to ensure the dynamic economic dispatch (DED) between generators of the system. The DED problem consists of allocating the total demand among generating units so that the production cost is minimized [238]. However, it is important to stress that the DED in the MG context is more straightforward than the DED in a conventional power system. Indeed, the MG has much fewer units than the whole system. Also, the DED in MG does not have a wide variety of conventional fuel generators and, at the same time, tries to integrate more renewable energy RE resources. The hypotheses considered in this work are as follows:

- The distribution of power losses neglected;

- The electric water heater is considered a controllable load;

- The reactive power flow is neglected;

- The MG is operating in connected mode.

The objective function of the DED optimization problem is defined as: 


$$
\min \sum_{t=1}^{m}\left(F_{t}^{p v}+F_{t}^{w}+F_{t}^{E S-}+F_{t}^{G-}-F_{t}^{G+}-F_{t}^{E S+}-F_{t}^{l}-F_{t}^{c l}\right) \cdot \Delta t
$$

$m$ represents the optimization period. $F_{t}^{p v}$ and $F_{t}^{w}$ represent the cost function of the PV panels and the wind turbine respectively; $F_{t}^{E S-}$ and $F_{t}^{E S+}$ represent the cost function of the battery storage in discharging and charging mode respectively; $F_{t}^{G-}$ and $F_{t}^{G+}$ represent the cost function of the power imported from the grid and exported to the grid respectively; $F_{t}^{l}$ and $F_{t}^{c l}$ represent the cost function of the non-controllable loads and a controllable load of the EWH, respectively. The generation cost function of each unit is determined by:

$$
\begin{gathered}
F_{t}^{p v}=\sum_{k=1}^{n_{p v}} \pi_{t}^{k, p v} P_{t}^{k, p v} \\
F_{t}^{w}=\sum_{k=1}^{n_{w}} \pi_{t}^{k, w} P_{t}^{k, w} \\
F_{t}^{E S-}=\sum_{k=1}^{n_{E S}} \pi_{t}^{k, E S-} \cdot\left(1-\theta_{E S}\right) \cdot P_{t}^{k, E S-} \\
F_{t}^{E S+}=\sum_{k=1}^{n_{E S}} \pi_{t}^{k, E S+} \cdot \theta_{E S} \cdot P_{t}^{k, E S+} \\
F_{t}^{G-}=\pi_{t}^{G-} \cdot\left(1-\theta_{G}\right) \cdot P_{t}^{G-} \\
F_{t}^{G+}=\pi_{t}^{G+} \cdot \theta_{G} \cdot P_{t}^{G+}
\end{gathered}
$$




$$
\begin{gathered}
F_{t}^{l}=\sum_{k=1}^{n_{l}} \pi_{t}^{k, l} P_{t}^{k, l} \\
F_{t}^{c l}=\sum_{k=1}^{n_{c l}} \pi_{t}^{k, c l} P_{t}^{k, c l}
\end{gathered}
$$

where $\pi_{t}^{k, p v}, \pi_{t}^{k, w}$ depict the offer prices by the $k$ th PV panel and the wind turbine respectively; $n_{p v}, n_{w}, n_{E S}$ indicate the number of PV panel, wind turbines, and ES installed in the MG; $\pi_{t}^{k, E S-}, \pi_{t}^{k, E S+}$ represent the electricity cost of the $k$ th storage unit during discharging and charging mode respectively; $\theta_{E S}$ is a binary number equal to 1 if the battery is charging and 0 otherwise; $\pi_{t}^{G-}, \pi_{t}^{G+}$ depict the power price of the power imported from the grid and exported to the grid respectively; $\theta_{G}$ is a binary number equal to 1 if the MG is exporting to the grid and 0 otherwise; $\pi_{t}^{k, l}, \pi_{t}^{k, c l}$ represent the power cost of the of non-controllable loads and a controllable load of the EWH, respectively. $n_{l}, n_{c l}$ indicate the number of the of non-controllable loads and controllable load; $P_{t}^{k, p v}$ is the power generated by the $k$ th PV panel during time period $t ; P_{t}^{k, w}$ is the power generated by the $k$ th wind turbine during time period $t ; P_{t}^{k, E S-}, P_{t}^{k, E S+}$ are the power generated and consumed off the $k$ th storage unit during discharging and charging mode respectively; $P_{t}^{k, l}$ and $P_{t}^{k, c l}$ are the power generated and consumed by non-controllable loads and a controllable load of the EWH respectively.

The objective function must be minimized subject to the following constraints:

- Power balance 


$$
\begin{aligned}
\sum_{k=1}^{n_{p v}} P_{t}^{k, p v}+\sum_{k=1}^{n_{w}} P_{t}^{k, w}+\sum_{k=1}^{n_{E S}} \theta_{k, E S} \cdot P_{t}^{k, E S-}+\theta_{G} \cdot P_{t}^{G-} \\
=\sum_{k=1}^{n_{E S}}\left(1-\theta_{k, E S}\right) \cdot P_{t}^{k, E S+} \\
+\left(1-\theta_{G}\right) \cdot P_{t}^{G+}+\sum_{k=1}^{n_{l}} P_{t}^{k, l} \\
+\sum_{k=1}^{n_{c l}}\left(1-\theta_{k, c l}\right) \cdot P_{t}^{k, c l+} \\
-\sum_{k=1}^{n_{c l}} \theta_{k, c l} \cdot P_{t}^{k, c l-}
\end{aligned}
$$

- The renewable energy resources

$$
\begin{aligned}
0 & \leq \sum_{k=1}^{n_{p v}} P_{t}^{k, p v} \leq P_{t}^{\max , p v} \\
0 & \leq \sum_{k=1}^{n_{w}} P_{t}^{k, w} \leq P_{t}^{\max , w}
\end{aligned}
$$

where $P_{t}^{\max , p v}, P_{t}^{\max , w}$ are the maximum available power from PV panels and wind generators during the period $t$.

- $\quad$ ES unit [239]:

Maximum discharge limit:

$$
\theta_{E S} \cdot P_{t}^{E S-} \leq P_{\max }^{E S-}, P_{t}^{E S-} \geq 0
$$


Maximum charge limit:

$$
\left(1-\theta_{E S}\right) \cdot P_{t}^{E S+} \leq P_{\max }^{E S+}, P_{t}^{E S+} \geq 0
$$

When the battery is in discharging mode $\theta_{E S}=1$, the power discharged cannot go beyond the maximum discharging power of the battery $P_{\max }^{E S-}$; similarly, the power charging the battery cannot go beyond the maximum power that could be consumed by the battery $P_{\max }^{E S+}$.

Maximum discharge limit considering the total stored energy:

$$
\left(\theta_{E S} \cdot P_{t}^{E S-} \cdot \Delta t\right) \leq E_{t-1}^{E S}
$$

- Maximum charge limit considering the total stored energy:

$$
\left(\left(1-\theta_{E S}\right) \cdot P_{t}^{E S+} \cdot \Delta t\right)+E_{t-1}^{E S} \leq E_{\max }^{E S}
$$

- Energy balance in ESS:

$$
E_{t}^{E S}=E_{t-1}^{E S}+\left(P_{t}^{E S+}-P_{t}^{E S-}\right) \cdot \Delta t
$$

The inequality (16) formulates the energy constraint for the battery in a way that the discharged should not exceed the available power. Similarly, the energy charging the battery should not exceed the upper battery limit. During the battery operation, the available energy $E_{t}^{E S}$ should always be between the upper and lower limits $E_{\text {min }}^{E S}, E_{\max }^{E S}$ respectively as shown in Equation (18) [240].

$$
E_{\min }^{E S} \leq E_{t}^{E S} \leq E_{\max }^{E S}
$$

- State of charge of the battery:

$$
S O C_{t}=\frac{E_{t}^{E S}}{E_{t o t}^{E S}}
$$


- The interconnection with the grid [30]:

$$
\begin{aligned}
& \left(1-\theta_{G}\right) \cdot P_{t}^{G+} \leq P_{\max }^{G+}, P_{t}^{G+} \geq 0 \\
& \theta_{G} \cdot P_{t}^{G-} \leq P_{\max }^{G-}, P_{t}^{G-} \geq 0
\end{aligned}
$$

$P_{\max }^{G-}$ is the maximum power that could be injected in the grid; $P_{\max }^{G+}$ is the maximum power that could be imported from the grid.

\subsection{Proposed Algorithm for the Real-Time Optimization}

Among classical techniques to solve the DED are the dynamic programming [242, 243] or the Lagrangian relaxation [215, 244, 245]. However, most deterministic optimization techniques are not suited for real-time optimization since they need more time to reach the optimum. Heuristic techniques, on the other hand, may reach a sub-optimum solution, but their computational time is very competitive, which make them adequate for real-time application. Much progress was made in heuristic techniques that have proven their efficiency as competitive optimization methods in the power system [214, 216, 218, 246 - 248]. The biological process of immune cell defense mechanisms inspires the artificial immune algorithm. The search process of this optimization approach is fast and robust, which, not like the particle swarm and the genetic algorithm, search for potential solutions in the overall shape space and do not fall into the trap of sub-optimality convergence [249]. This work proposes the T-Cell algorithm as a variant of the immune system algorithm to solve the DED in real-time MG operation. 
The T-Cell algorithm is inspired by the mediated immune cells in the human body. These cells are called lymphocytes and develop in the Thymus as a group of white blood cells [225]. They play a central role in cell-mediated immunity. They have receptors called T-Cell Receptors (TCR) that interact with proteins in their environment. Through this interaction, the T-Cells suffer two stages to get activated: proliferation then differentiation. In the first process, T-Cells proliferate by generating other clones of themselves; then, in the second process, each clone cell acquires new propriety; this is the so-called activation process [226]. This algorithm implements this process:

Each cell has nine characteristic values:

- $P_{t}$ represents the decision variables of the dispatch problem;

$$
P_{t}=\left[\begin{array}{ccc}
P_{t}^{1, p v} & \ldots & P_{t}^{n_{p v}, p v} \\
P_{t}^{1, w} & \ldots & P_{t}^{n_{w}, w} \\
P_{t}^{1, E S-} & \ldots & P_{t}^{n_{E S-}, E S-} \\
P_{t}^{1, E S+} & \ldots & P_{t}^{n_{E S+}, E S+} \\
& P_{t}^{G-} \\
& P_{t}^{G+} \\
P_{t}^{1, c l-} & \ldots & P_{t}^{n_{c l-}, c l-}
\end{array}\right]
$$

- The objective function value for $P_{t}$;

- The proliferation is the number of clones that the cell will generate;

- The differentiation is the number of decision variables that will be changed when the differentiation process takes place;

- The power generated by $P_{t}$;

- $\quad$ The equality constraint violation $(\mathrm{ECV})$ for $P_{t}\left(\left|P-P_{l}\right|\right)$; 
- The inequality constraints violation (ICV) sum;

- The feasible propriety indicates if the cell is feasible or not. A cell is considered as feasible if:

$\mathrm{ECV}=0$ and $\mathrm{ICV}=0$. This means that if a solution generates less than the demanded power $(\mathrm{ECV}<0)$ or more than needed $(\mathrm{ECV}>0)$, or the decision variable are in a prohibited zone $(\mathrm{ICV}<0)$ or $(\mathrm{ICV}>0)$ then it is considered as infeasible.

The required parameters for the T-Cell algorithm are the Number of objective function evaluations, population size and probability of the redistribution operators. The appropriate values of each parameter were chosen from the analysis result of this study [216].

Figure 7.1 describes the T-Cell algorithm execution steps.

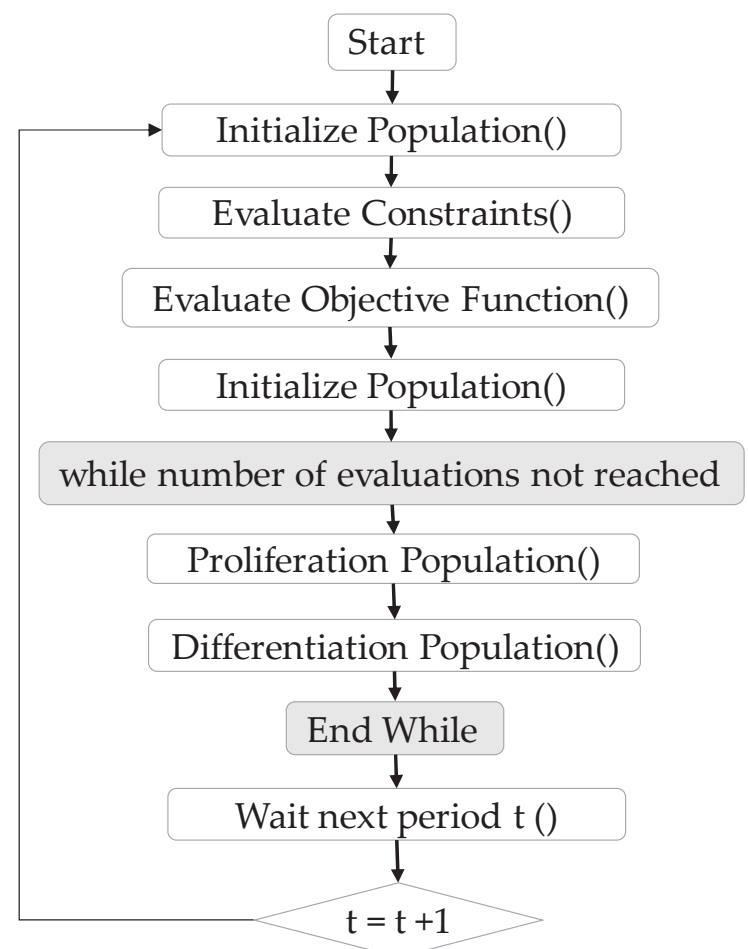

Figure 7.1 T-Cell algorithm process. 


\subsection{Proposed Multiagent EMS Platform}

This section presents the MAS implemented for MG management and will present the developed agents and the proposed management strategy.

The agents are implemented in the Java Agent Development Framework (JADE) platform. This platform respects the Foundation for Intelligent Physical Agents' (FIPA) standards that are considered as the reference for MAS standards used by computer science developers' community. JADE platform has the default Agent Management System (AMS) that controls the platform, creates and destroys other agents.

The default Directory Facilitator (DF) is the agent responsible for registering all connected agents of the platform. Thus, if another agent wants to communicate with his peer, he has to ask the DF who will provide him with the name of the connected agent. In addition to these agents, the Agent Communication Channel, also called the Message Transport System, is the agent in charge of transferring messages between agents of the platform [250].

JADE also provides graphical management service for platform management and control through the remote management agent (RMA). Any agent created in JADE is hosted in a container. This latter has all services for executing and hosting agents. Figure 7.2 presents the structure of the JADE platform, according to the FIPA agent management reference model. 


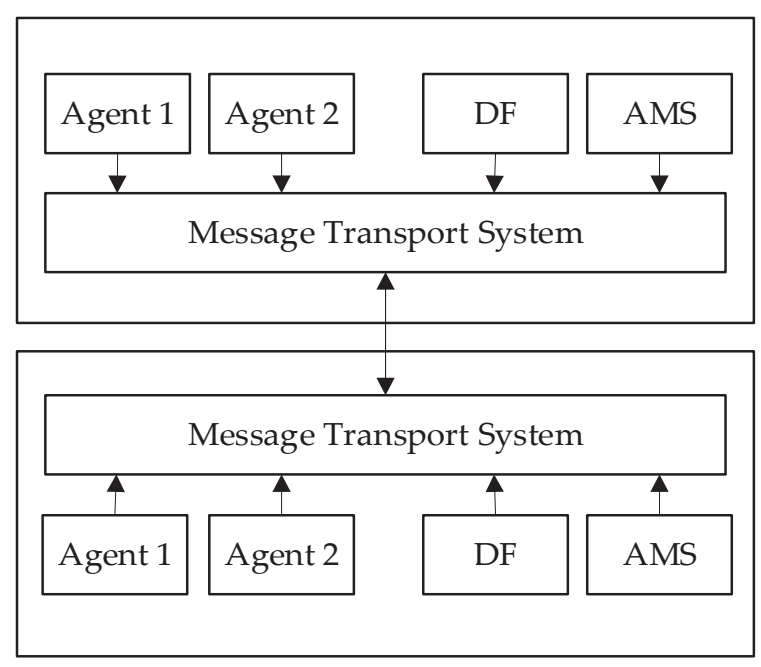

Figure 7.2 Jade platform architecture

The MG considered in this work is composed of wind generators, PV panels, storage units, controllable load, and non-controllable load. The MG is operating in grid-connected mode. Each one of these components has a specific agent. There are three agent categories: The management agents, backup agents, and communication agents.

The MAS implemented is illustrated in Figure 7.3.

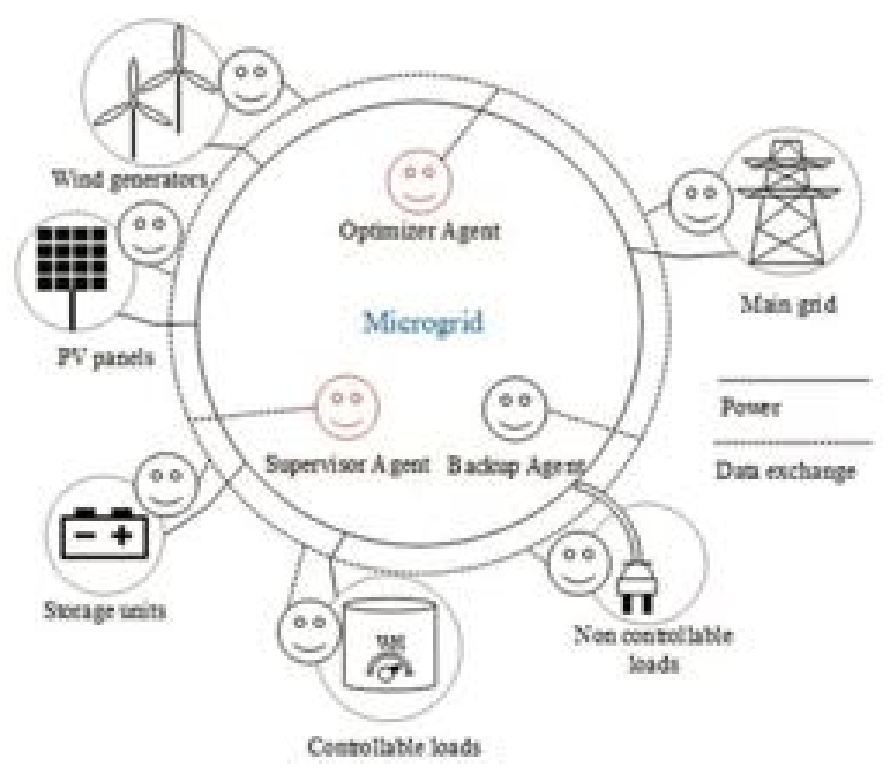

Figure 7.3 MAS architecture. 


\subsubsection{Management Agents}

The first category comprises agents that ensure the proper operation of the MG. The MG optimizer (MGO) is the agent responsible for running the optimization and ensuring the seamless operation of the MG in real-time. The T-Cell algorithm is implemented in this agent. This agent gets the forecast data of the wind, PV, and load power and computes the references of each generation unit and controllable load at each time step $15 \mathrm{~min}$. However, during this period, an unpredictable load variation can occur, which can jeopardize the MG operation. So, instead of running the optimization every few seconds, which is resource consuming, this work presents a solution to implement a specific agent that checks continually if the load power has gone beyond permissible limits during the time step.

The MG supervisor (MGS) is the load supervisor agent. When the MGS intercepts sudden high load variations, it contacts the MGO that launches a new optimization cycle and computes the new optimum operating points of the generators and controllable loads

\subsubsection{Backup Agent}

The Main Container (MC) is the special node which the agent platform relies on. It coordinates between the other nodes and hosts the AMS and the DF. Since this node is very critical, if it terminates or fails to connect to other containers, some vital operational features can become unavailable, and this severely hampers the platform [251]. In order to strengthen the agent platform and make it more reliable, a fault-tolerant structure is implemented. It can be observed from Figure 7.4 that there are two $\mathrm{MC}$ in the agent platform. Replication support for MC has been introduced. Using this support, it is also possible to duplicate as many as $\mathrm{MC}$ in the system. These $\mathrm{MC}$ arrange themselves in a 
logical ring so that whenever one of them fails, the others will notice and act accordingly [229].
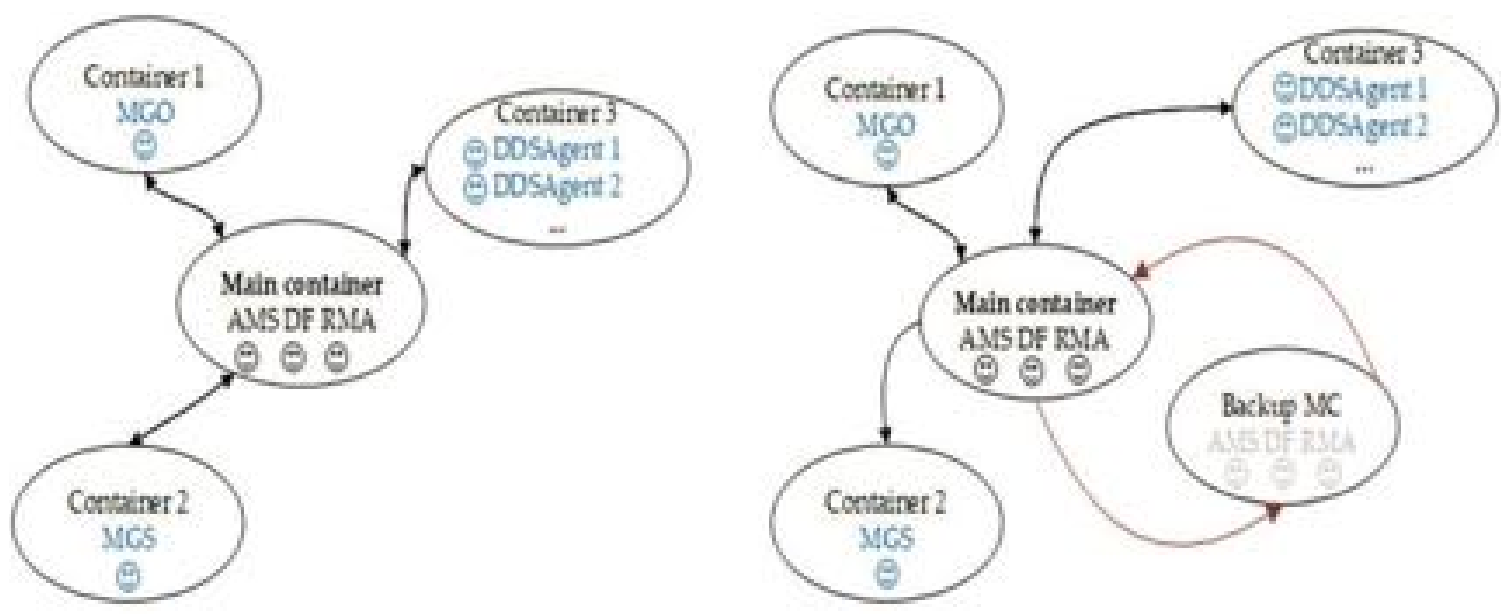

Figure 7.4 The fault tolerance mechanism in the MAS platform.

\subsubsection{Interoperability Agents}

The main function of interoperability agents is to ensure the communication between the MAS platform and the physical hardware. The communication is based on the datacentric publish-subscribe (DCPS) model.

Two main distributed communication models could be implemented in the MG context: Data-centric communications and object-centric communication models. In data-centric communications, data distribution between communicating entities is the fundamental concept. This model is based on data publishers and data subscribers. Publishers pass data of known types to subscribers through a common channel. In the opposite, in objectoriented communications, instead of focusing on the data, the main concern is the interface between entities. 
An interface comprises of a list of predefined methods ready to be used. An objectoriented system is based on server and client interface communication. The client interface invokes methods on a named server interface, in which the server responds accordingly [252]. Even though the two approaches are complementary, middleware built on datacentric communication is more competitive in the RT applications.

RTI DDS Connect was implemented as middleware for RT interoperability between the agent context and the hardware of the testbed. As explained earlier, data-centric communication is based on data sharing between publishers and subscribers. The RTI DDS Connext allows to user to create publishers and subscribers independently of the programming language.

Publishers and subscribers are connected by 'Topics.' A Topic corresponds to a single data type that allows identifying which data is being to be shared between which publishers and subscribers [253]. For instance, if publisher $A$ is publishing value ' $\mathrm{x}$ ' in Topic 1, subscribers who want to get the ' $\mathrm{x}$ ' value should connect to the same Topic 1.

In this work, a publisher for each generation unit and controllable load were implemented in the MAS context. The MGO computes the references and sends them to the corresponding publisher agents.

Once the data is published, the subscribers implemented in the SCADA system of the testbed read the data and operate accordingly. Figure 7.5 illustrates how the agents implemented in the agent context publish data in RTI DDS topics, and how each generation unit reads the published data through subscribers. 


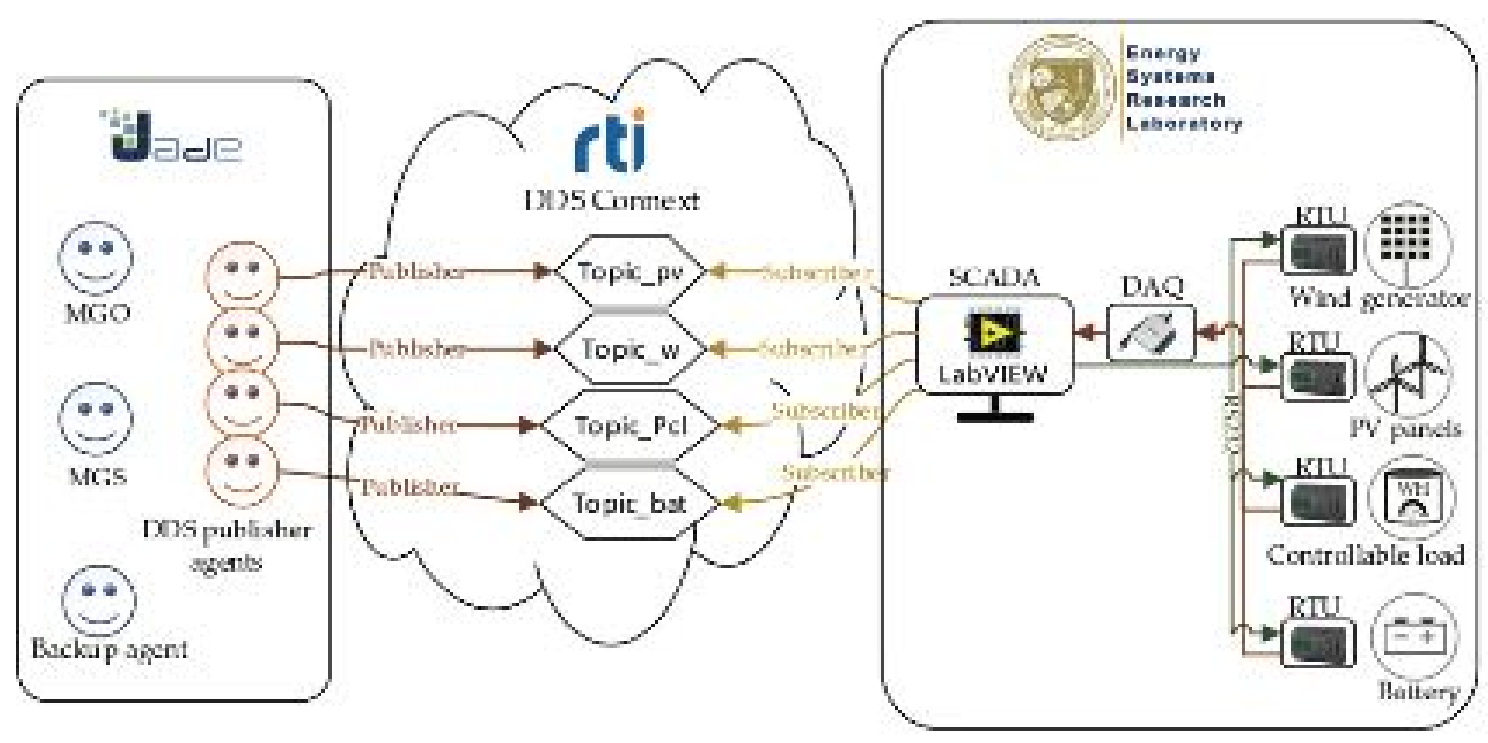

Figure 7.5 Interoperability Communication Setup

\subsection{Results}

\subsubsection{Description of the Cyber-Physical Setup}

This section describes the implemented configuration of the testbed for the multi-agent RT-EMS experimentation in the Energy Systems Research Laboratory (ESRL) testbed, Florida International University [254].

The MG considered in this study is composed of a wind generator and a synchronous generator, a storage unit emulated by a DC power supply, a controllable load, load, and connection to the grid, emulates PV system, each one.

Each generating station has five different types of motor drives, acting as five different prime movers to enable the implementation of various generation control strategies. These generators are equipped with frequency drives, Automatic Voltage Regulator (AVR) and synchronizer systems to control these different prime movers. The voltage regulators and automatic power control module controllers enable the system to operate as real-time 
generators. An automatic synchronizer connects each generator and controls the switching and measurement busses. The control and setup of each generation station are described in Figure 7.6.

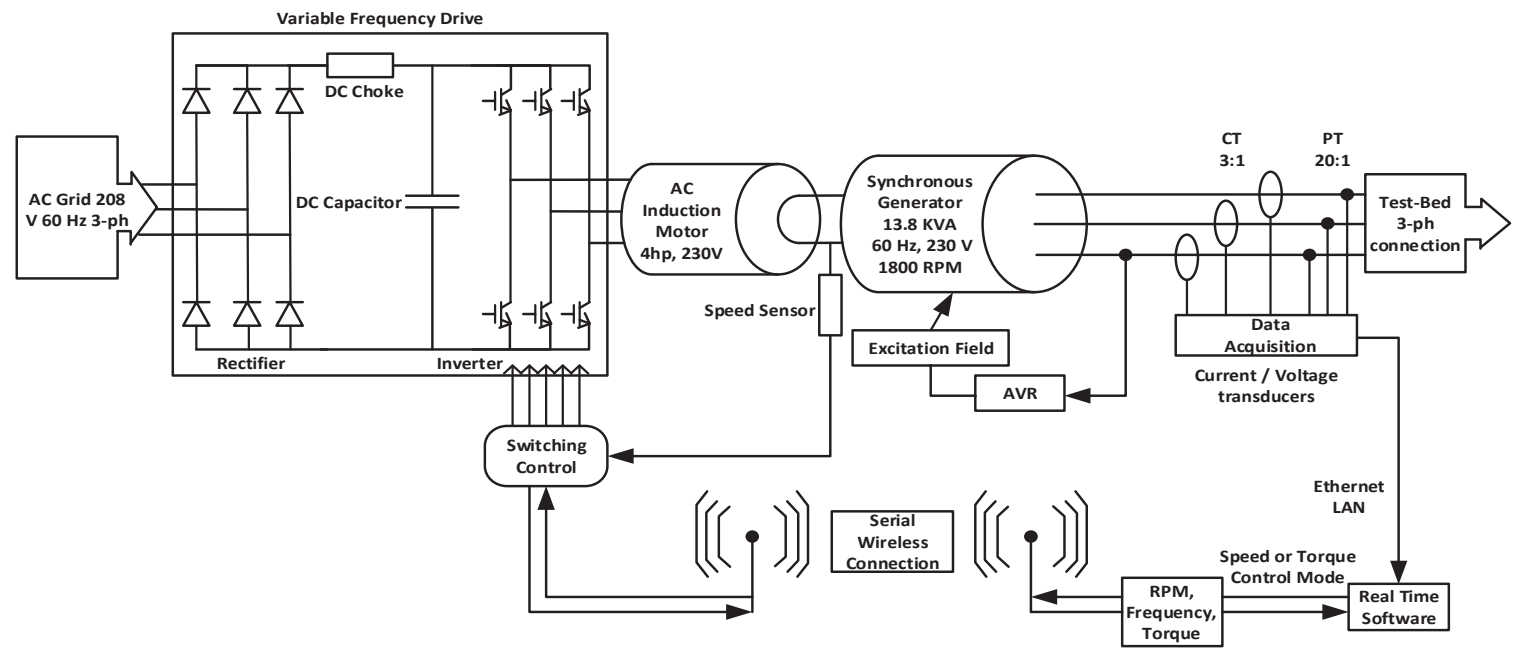

Figure 7.6 Generation station hardware and control

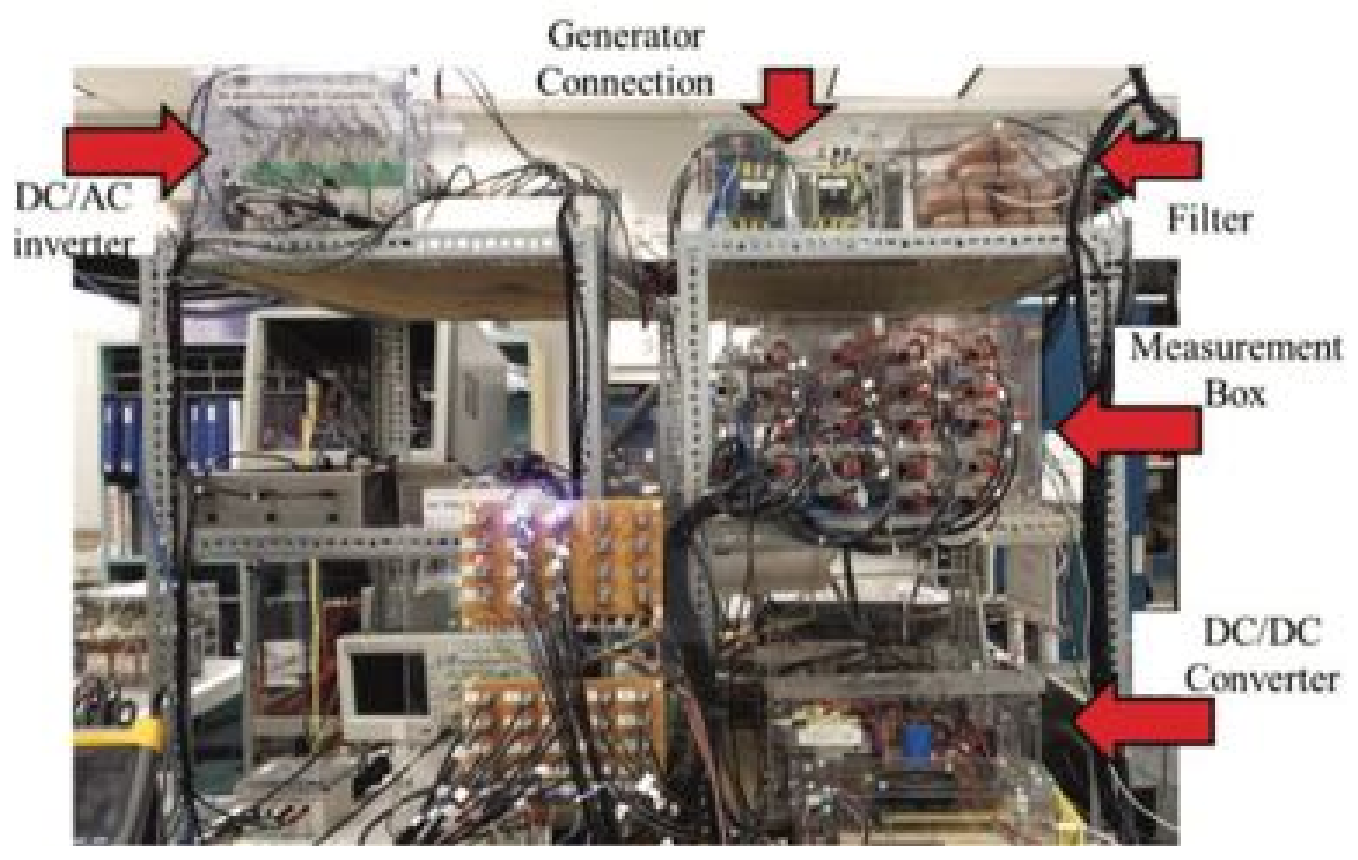

Figure 7.7 Control of DC power supply 
A DC bus represents the DC zone that emulates the DC storage unit, where its voltage was set to $380 \mathrm{~V}$ and XR SERIES DC power supply offered by the MAGNA-POWER ELECTRONICS, which emulates the charging/discharging battery characteristics.

It is interfaced with the DC bus through the DC-DC boost converter. The converter controllers are implemented in the SIMULINK environment and executed in the DSpace 1104 real-time interface. The system topology can be changed by using eight controlled switches. The switches have two states on and off.

A control signal is sent to these switches to change their state, which changes the equivalent circuit. The control is initiated from a virtual instrument (VI) program implemented in the LabVIEW environment.

The card PCI 6025E sends the control command to the circuit. This circuit is an inverting buffer module sn7406n of TEXAS INSTRUMENT. This module contains six inverters with open collector output. The RMS values for the voltage in the AC zone is set to $208 \mathrm{~V}$. The AC bus is connected to the utility grid at PCC. Also, different load models were designed to represent the AC load pattern. Figure 7.7 shows the control of the power supply.

The testbed is controlled and monitored through the LabVIEW SCADA interface, as shown in Figure 7.8. The connected components for this experiment have switched with light green color. 


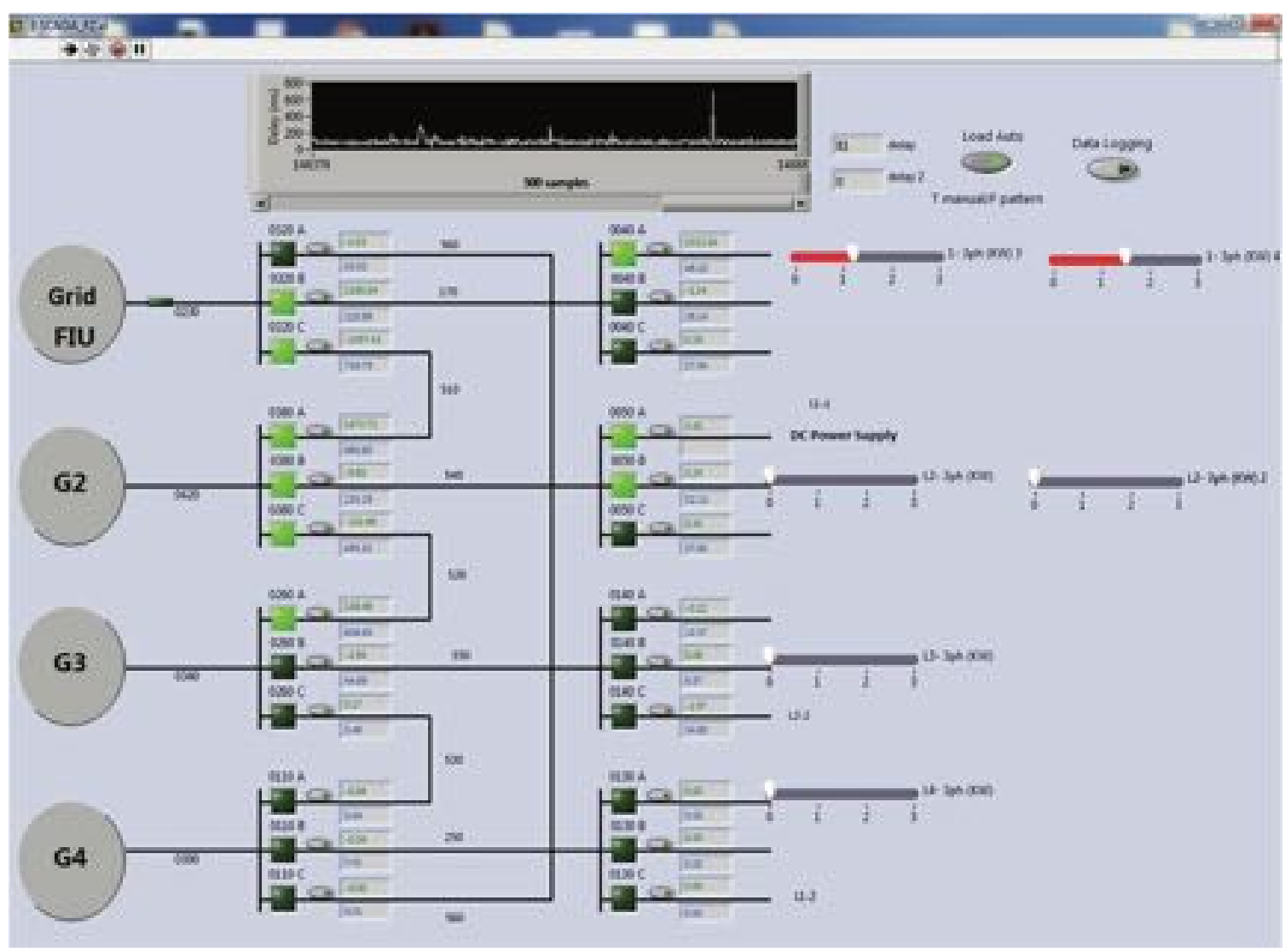

Figure 7.8 SCADA interface in ESRL

\subsubsection{Experiment Results}

This section presents the results obtained from simulation and experimentation of the multi-agent RT-EMS. Three case studies are considered. The first case study presents the implemented load supervision mechanism of the MAS.

The second case study describes the operation of the fault tolerance mechanism in the MAS. The third case study compares the results of the experimentation and the simulation and presents the competitively of the implemented RT-EMS in terms of time delay. 


\subsubsection{Case Study 1}

This case study presents the implemented RT load supervision mechanism. The MGO conducts the optimization every 15 min based on the forecasted PV, wind, and load data. However, due to some exceptional event, the load value can occasionally make a high variation from the forecast value, which affects the stability of the MG operation. For this reason, the MGS was conceived to supervise the high load variation in RT. The MGS is a cyclic agent that executes every $2 \mathrm{~s}$. It computes the variation between the RT load and the forecast values. When the computed difference exceeds $30 \%$, the MGS sends a signal to the MGO to start another optimization cycle based on the new sensed load value.

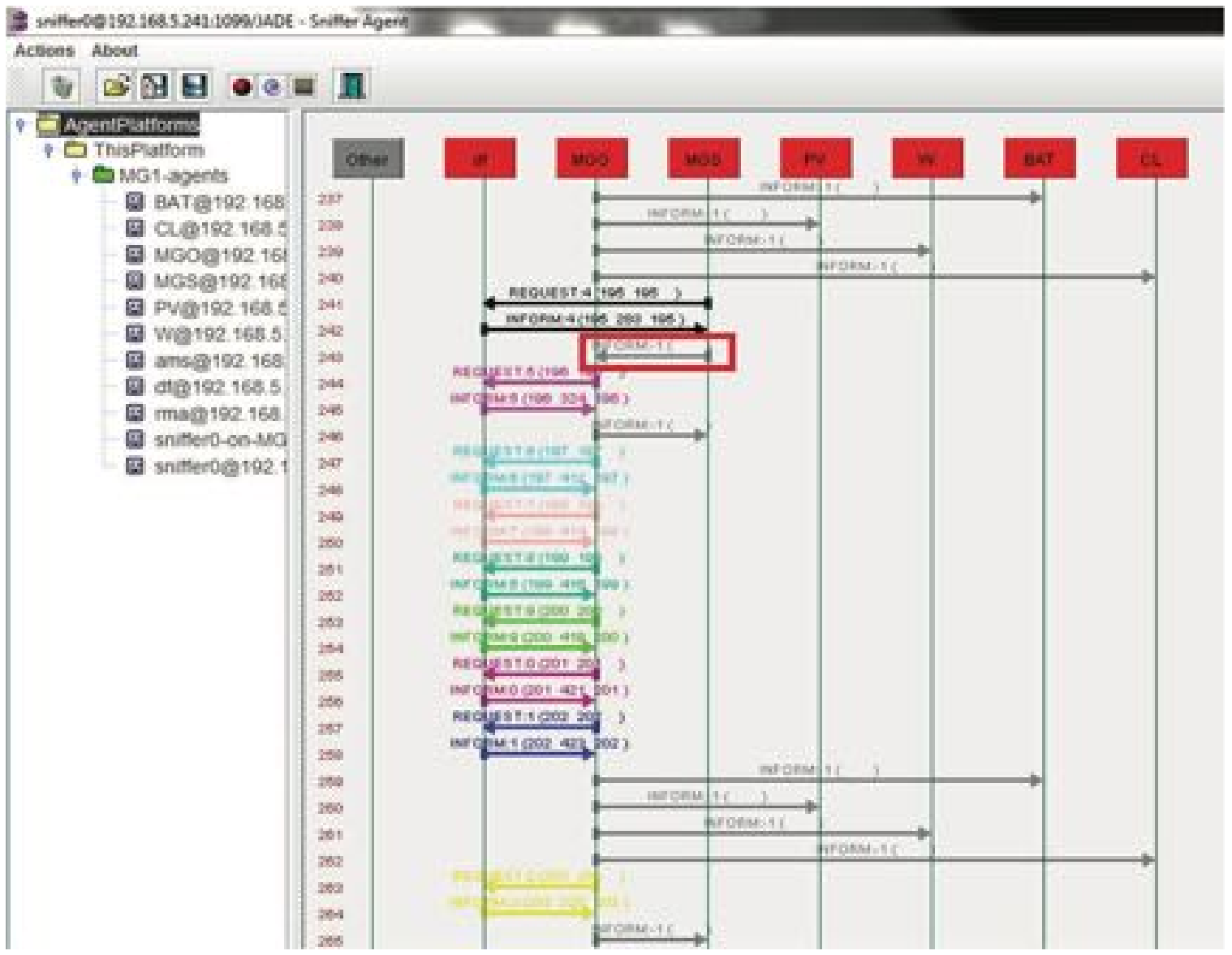

Figure 7.9 Communication between MGO, MGS and other agents 
Figure 7.9 shows the communication mechanism implemented in the Jade platform between the MGO, MGS, and other publisher agents.

At line 241, the MGS detects a high variation of the load. Then, the MGS sends a request to the DF agent to send him references of connected MGO. Once credentials are

delivered, the MGS sends it to the MGO, the signal to start a new optimization cycle at line 243. An optimization communication cycle starts from line 244 to line 262. The MGO at the beginning of each cycle sends the parameters of the current cycle to the MGS. Then, the MGO launches the optimization based on the T-Cell algorithm. At the end of the optimization, the computed references are sent to the publisher agents.

\subsubsection{Case Study 2}

This case study describes the implemented operation of the fault tolerance mechanism in the MAS. As explained in Section 3, the Main Container (MC) is a special node in the JADE platform that coordinates between other containers and agents hosted in these containers. In order to implement a fault-tolerant mechanism, a backup agent of the MC was created. The two main-containers are arranged in a unidirectional ring. The MC is considered as the master MC, while the backup agent is considered as the slave. Once the master MC fails, the slave $\mathrm{MC}$ detects the failure and takes the appropriate recovery actions. Three containers were created in this case study. By default, the AMS and DF agents are affected by the master MC, which is the 'Main-Container' in our case. The 'BackupContainer' is created, and 'Container-1' hosts the MG agents. 'Mg1' is the MGO while other agents are the interoperability agents of each MG component. Figure 10 illustrates the MAS platform before and after the fault test. A manual failure event was 
introduced while the RT-EMS was running. Through the Jade user interface, the MC was terminated. Automatically and instantaneously, the backup agent detects the event, changes its status to the new master MC and transfers the AMS and DF from the faulty MC to its container. As a consequence, the list of all alive agents and containers in the multi-agent platform remains updated, and the RT-EMS continues its regular operation.

\subsubsection{Case Study 3}

This case study compares the results of the conducted experimentation and the simulation of the RT-EMS and presents the performance of this system compared to previous work.

The experimental validation of the implemented T-Cell based RT-EMS developed in a MAS environment was tested in the Energy Systems Research Laboratory at Florida International University. Three different scenarios were taken into consideration to validate the efficiency and performance of the proposed algorithm:

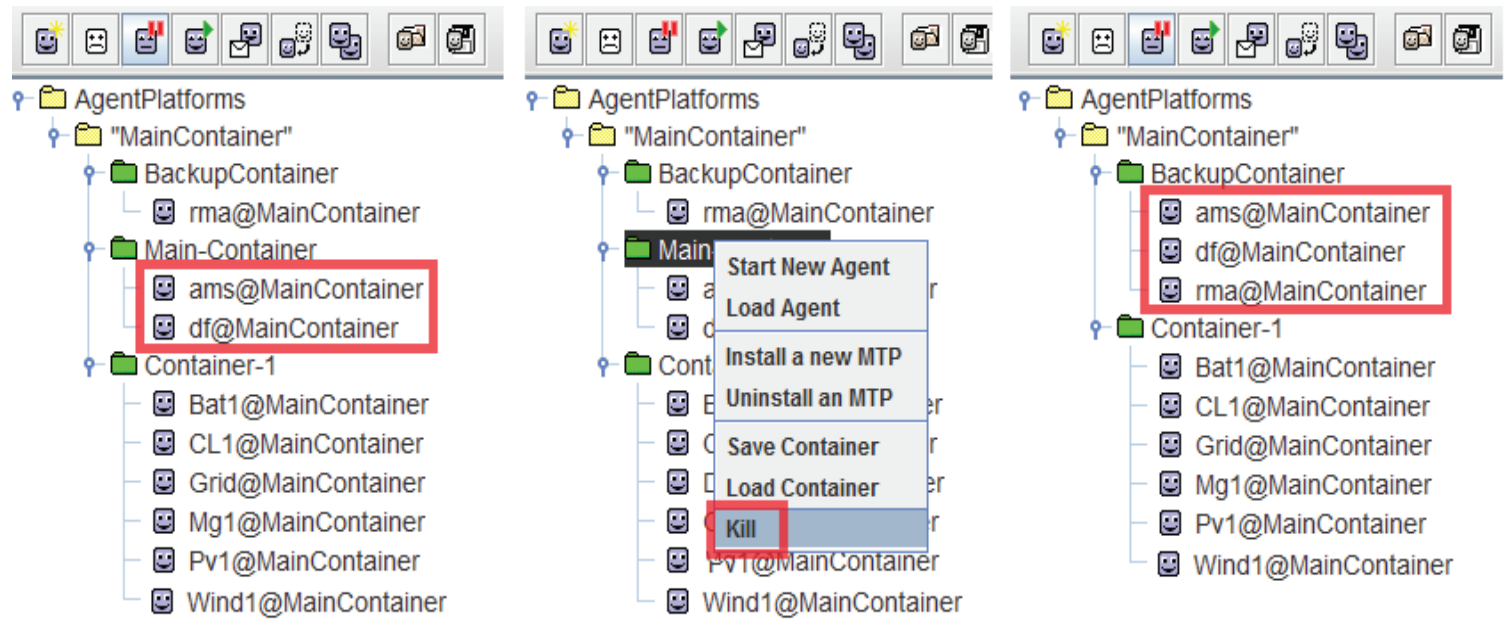

Figure 7.10 Fault tolerance mechanism in the JADE platform 
- Scenario 1: Normal operation of the MG. In this case, the system is running without a sudden event. The MGO is computing and sending references of the generation units according to forecast data without any incident

- Scenario 2: Generation drop. This case tests the ability of the system to operate after a significant shift in power generation. For this matter, the wind generator has been shut down during the period 6:00 -7:00;

- Scenario 3: Sudden load variation. This scenario challenges MG operation when there is a sudden load increase. It is reflected in the period 15:00-16:00, where the load increases from 1500 to $2400 \mathrm{~W}$.

Table 7.1 presents the parameters of the considered system. It presents the interval values of each system variable. The energy storage is considered operating between 0.4 and 0.9 to protect the battery from the deep discharging and overcharging

Table 7.1 Microgrid parameters.

\begin{tabular}{ccc}
\hline Variable & Meaning of the Variable & Limit Values \\
\hline $\mathrm{P}_{\mathrm{pv}}$ & The predicted power generation of the PV system & $0 \mathrm{~W} \leq \mathrm{P}_{\mathrm{pv}} \leq 700 \mathrm{~W}$ \\
$\mathrm{P}_{\mathrm{w}}$ & The predicted power generation of the wind turbine & $0 \mathrm{~W} \leq \mathrm{P}_{\mathrm{w}} \leq 1500 \mathrm{~W}$ \\
$\mathrm{P}_{\mathrm{bat}}$ & The predicted charging or discharging power of the & $-1500 \mathrm{~W} \leq \mathrm{Pbat}_{\mathrm{b}} \leq 1500$ \\
$\mathrm{SOC}$ & battery & $\mathrm{W}$ \\
$\mathrm{P}_{1}$ & State of charge of the battery & $0.2 \leq \mathrm{SOC} \leq 0.9$ \\
$\mathrm{P}_{\mathrm{cl}}$ & Active power load & $0 \mathrm{~W} \leq \mathrm{P}_{1} \leq 2700 \mathrm{~W}$ \\
\hline
\end{tabular}

Figures 7.11 and 7.12 illustrate the power profile of the wind and PV systems. As explained in Section 4.1, both wind and PV were emulated by two synchronous generators. 


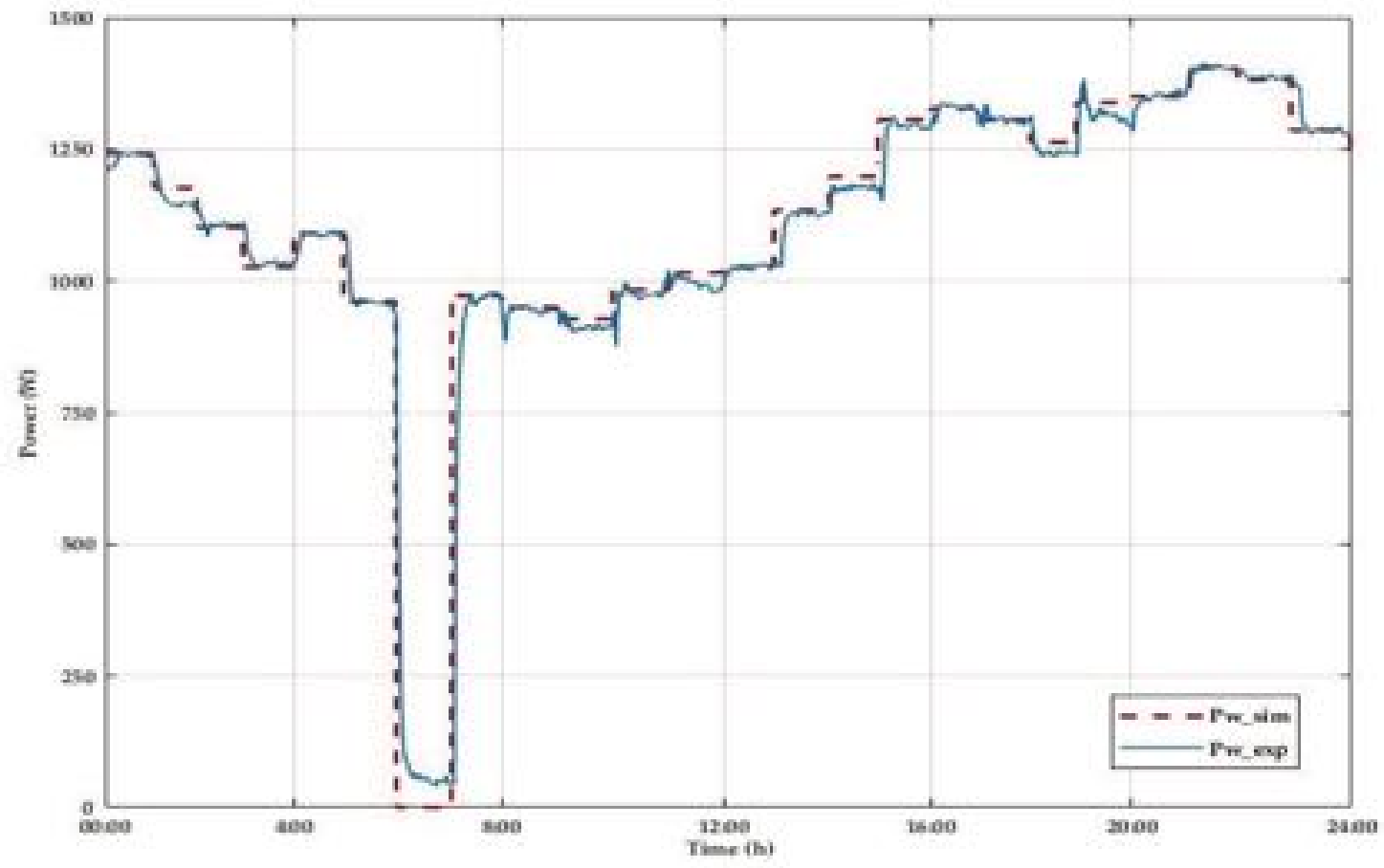

Figure 7.11 Comparison between simulated output and testbed measured values of wind power

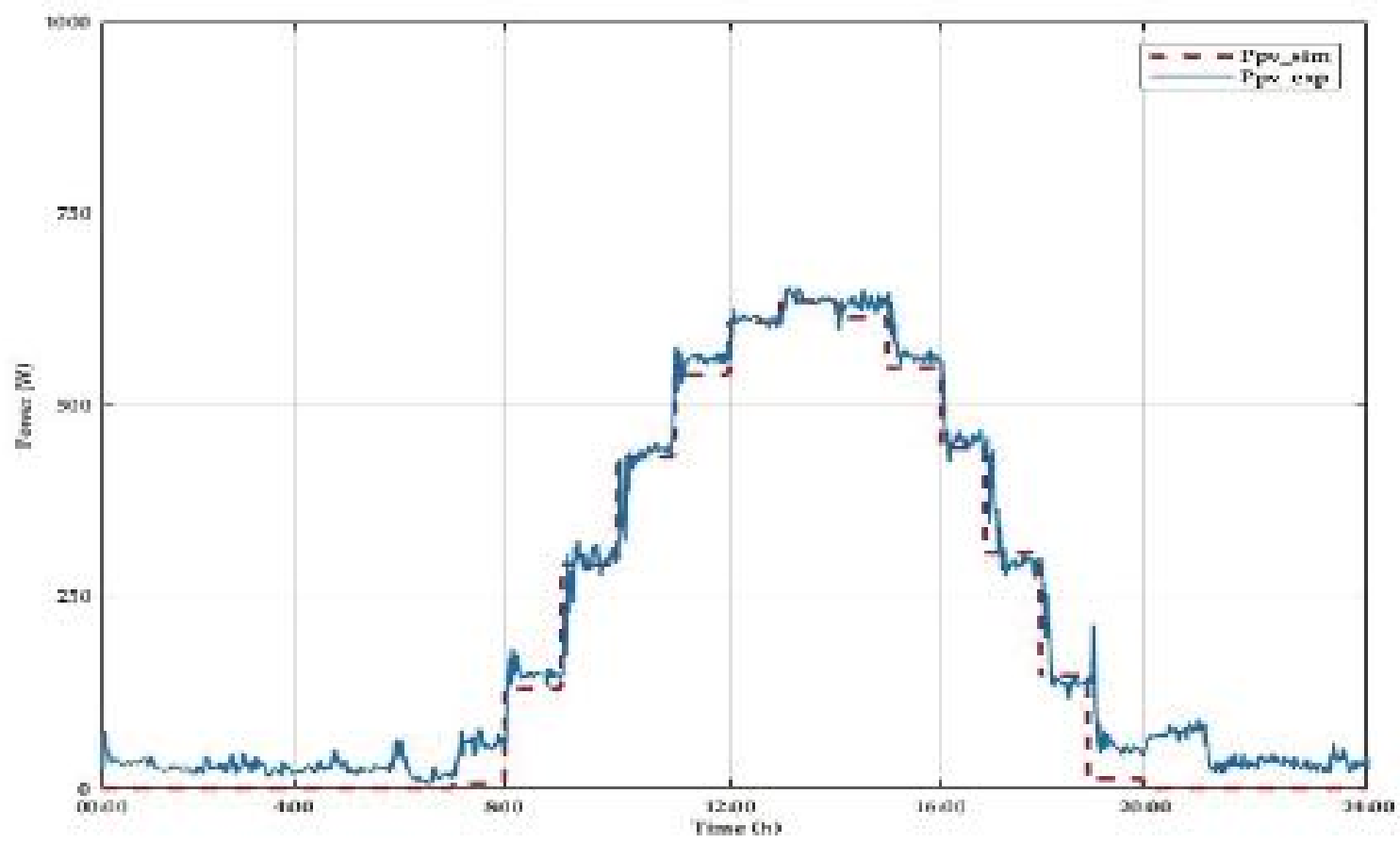

Figure 7.12 Comparison between simulated output and testbed measured values of PV power 
It is noticeable from the two profiles that at the beginning of each period, there is a power fluctuation due to system transients. The power measured during the experiment is also slightly below simulated values because of the system losses. The wind generator was supposed to shut down during the period 6:00-7:00 and the PV power profile was also assumed to be null before 7:00 and after 20:00, yet due to experimental constraints, the generators were kept running at the minimum level. However, in Figures 7.13, 7.14 and 7.15 , the experimental profile of the energy storage, the load and the controllable load matches the simulated values. The wind and PV profiles data used in this experiment are available in [254] and [255], respectively.

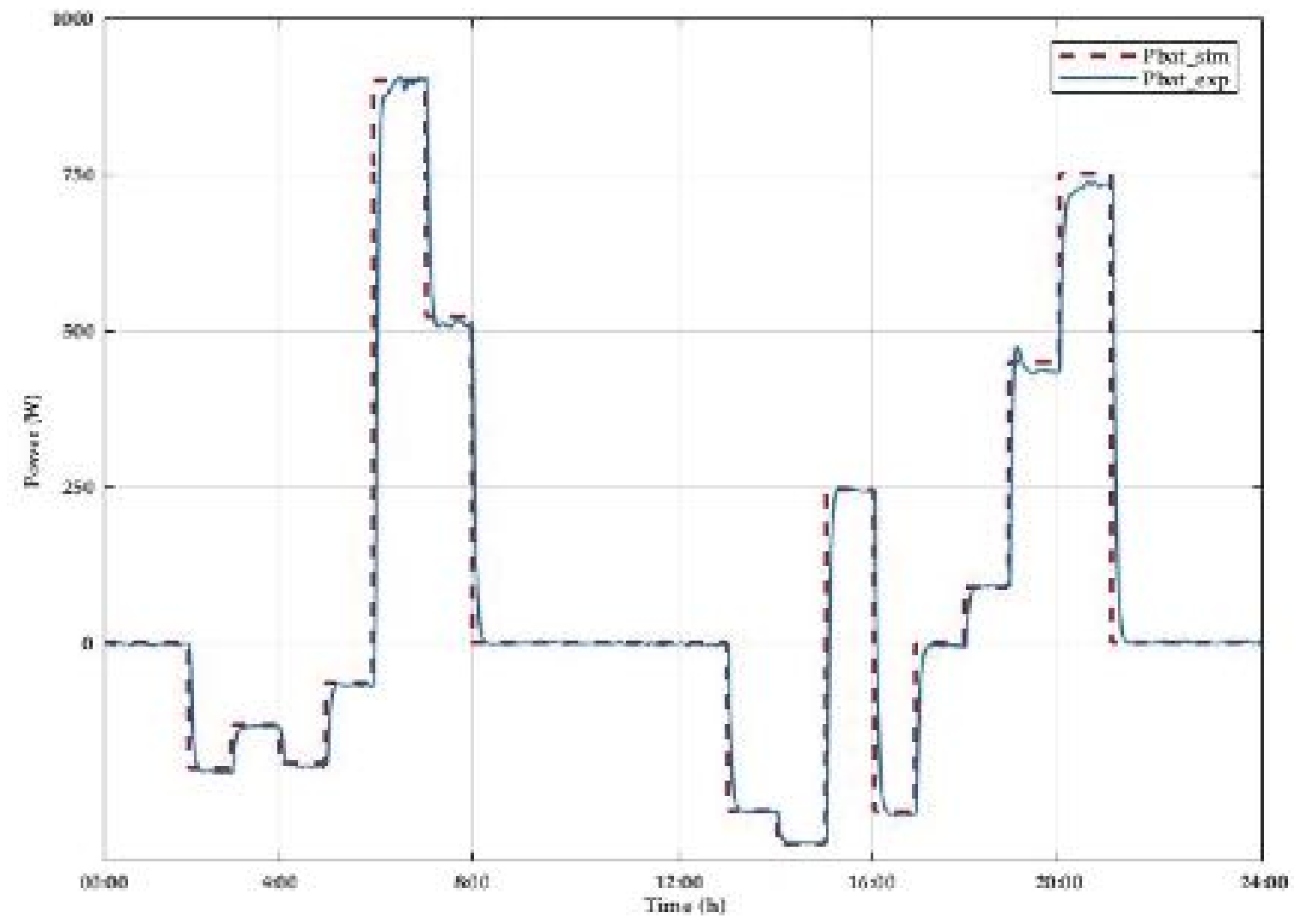

Figure 7.13 Comparison between simulated output and testbed measured values of energy storage power. 


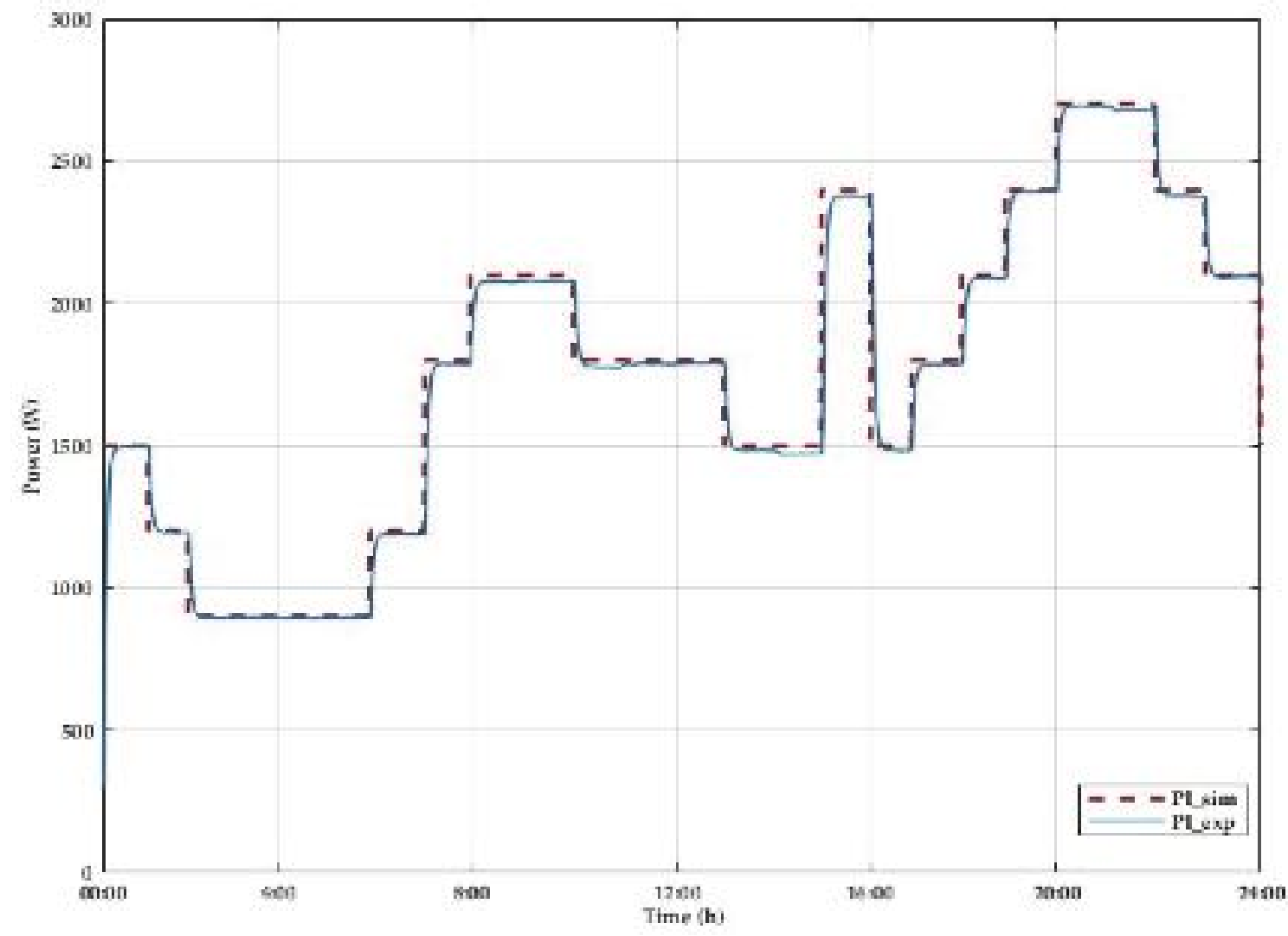

Figure 7.14 Comparison between simulated output and testbed measured values of load power

Figure 7.16 describes the power exchange with the grid. Unlike the PV and the wind emulators that receive the reference from EMS, the power exchange with the grid is automatically imported or injected depending on the system consumption and generation. For this reason, all system errors are compensated by the grid power, which creates, in some cases, a mismatch between the experiment and simulation values. It also should be mentioned that the simulation shows the RMS value without any transient, but the experimental result demonstrates the actual values along with transient of the power system which includes the compensation within the generators, reactions and power grid losses. 


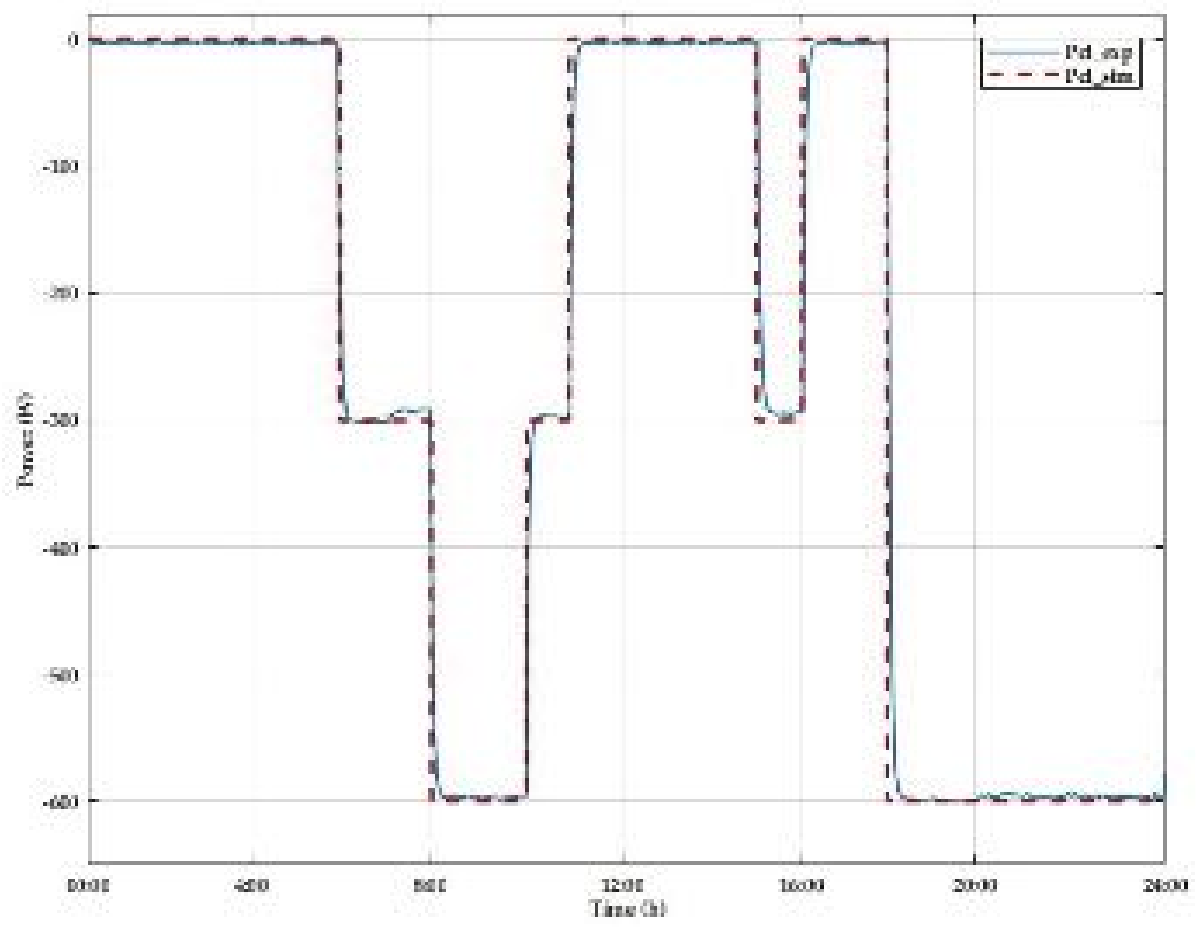

Figure 7.15 Comparison between simulated output and testbed measured values of controllable load

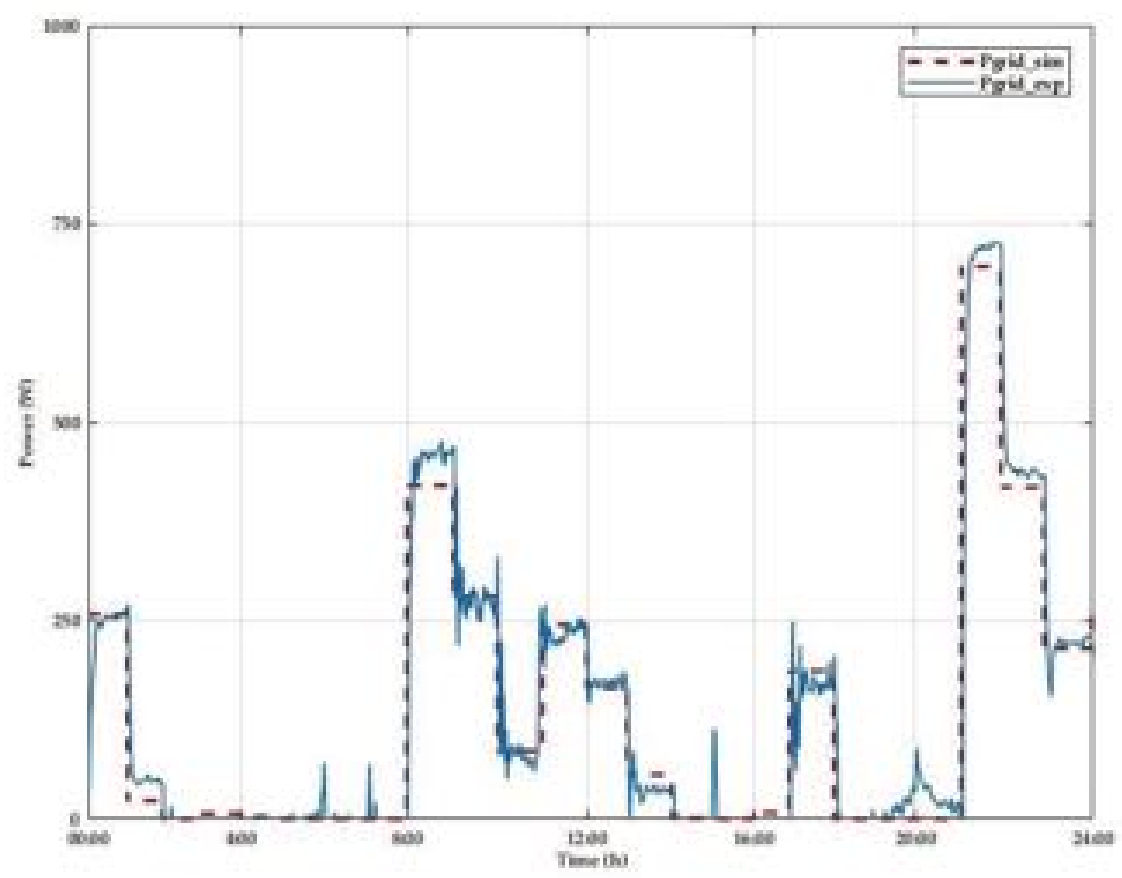

Figure 7.16 Comparison between simulated output and testbed measured values of grid power 


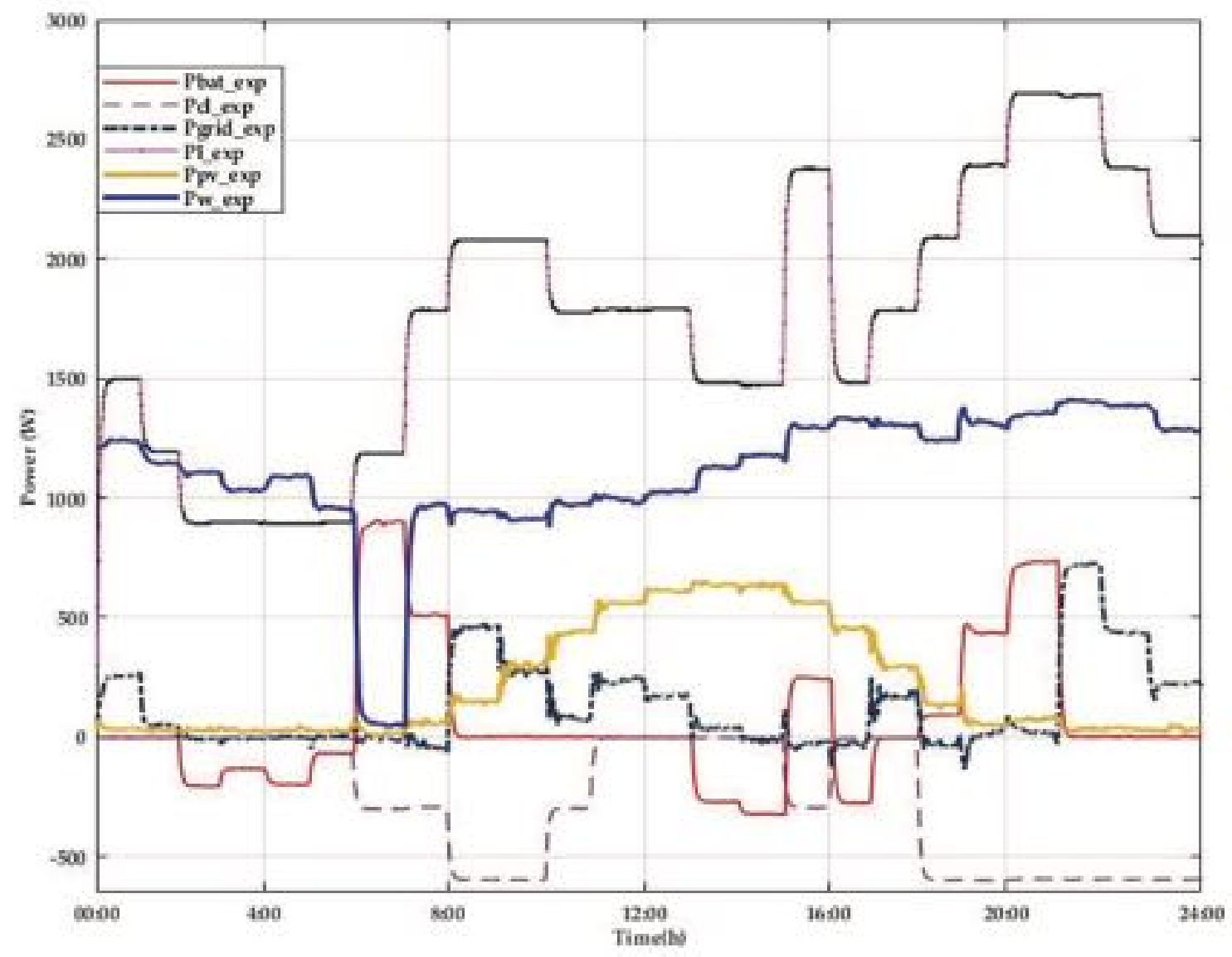

Figure 7.17 Experimental results of the MG powers during $24 \mathrm{~h}$ operation

Figure 7.17 illustrates the MG dynamic during a day of operation going through the three scenarios. From 00:00 to 2:00, the MG is supplying the load from the wind and the grid. As soon as the load decreases from 2:00 to 6:00, the proposed algorithm allows the charging of the energy storage with excess power. During the shutdown of the wind generator from 6:00 to 7:00, reflected by a drop of the wind power (scenario 2), the needed power was supported by the energy storage and the controllable load. The following day's hours match more load increase, which is supplied by the grid, wind, controllable load, and the increasing PV power. From 13:00 to 15:00, the PV power is at the top generation, which allows charging the energy storage with excess power. Scenario 3 is tested from 15:00 to 
16:00 in which the load suddenly rises from 1500 to $2400 \mathrm{~W}$. The algorithm compensates the deficit power using the energy storage and controllable load. The MG continues its normal operation and uses the available power from the energy storage until it reaches its minimum value; then, the power needed is imported from the grid and the available wind power. Through the $24 \mathrm{~h}$ of operation in different scenarios, the proposed T-cell based RTEMS has proven his effectiveness and capability of maintaining the stability and balance of the MG.

The proposed T-Cell algorithm that has been implemented for the EMS optimization is coded in Java language using the Eclipse programming environment. The environment integrates the JADE tool kit and the DDS interoperability agents. Before implementing those agents, it is important to define the data model for the MG. In our case, the data model consists of four variables of type double that represents the reference computed by the MGO. These four variables are published through the DDS agents into four topics, as illustrated in Figure 7.18. On the other side, the LabVIEW SCADA system implements the subscribers for these topics. Once the new data is published, it is automatically intercepted by the subscribers and sent to the appropriate generator or controllable load.

The RT-EMS has been tested using a computer with the following specifications: 4GB of RAM and $2 \mathrm{GHz}$ of CPU. In this configuration, the average execution time of the T-Cell algorithm is $231 \mathrm{~ms}$. However, when executed in a computer of $6 \mathrm{G}$ of RAM, the average execution time is $60 \mathrm{~ms}$. In both cases, the T-Cell algorithm presented better performance than mixed integer nonlinear programming (MINLP) and multi-period artificial bee colony algorithm (MABC) that have $8.23 \mathrm{~s}$ and $1.14 \mathrm{~s}$, respectively [221]. 


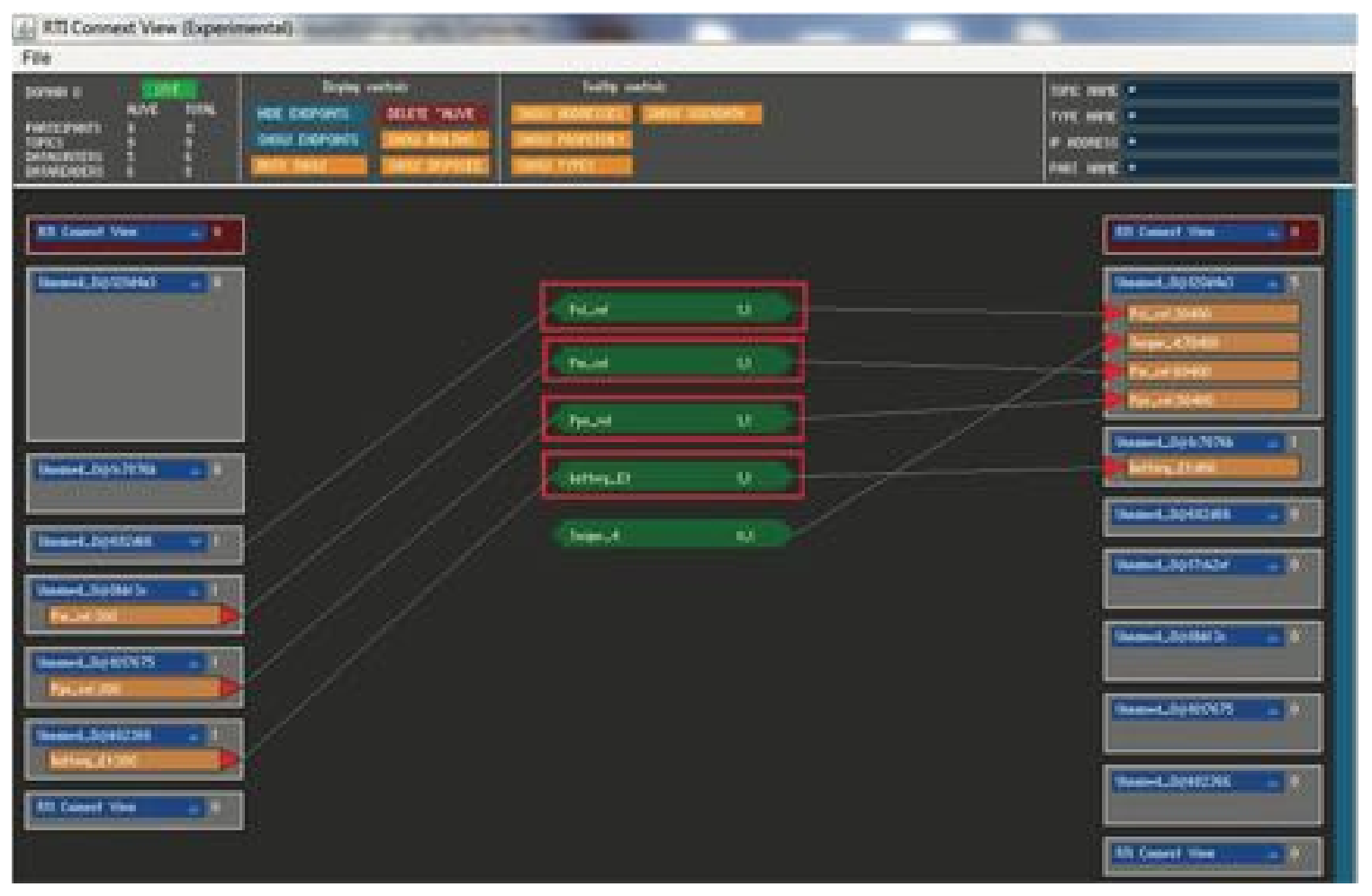

Figure 7.18 DDS Connext view

The execution time is very crucial for RT-EMS in the MG context and not as crucial as in day ahead management, where the optimization is run a day before. The notion of real-time that has been reported in different papers is between 3 to $5 \mathrm{~min}$. The reason behind choosing this time is because it is less than the time needed for the adjustment of protective relay such as over/under voltage relay. Besides, PV systems and wind generators usually need more than $5 \mathrm{~s}$ to be affected by atmospheric variations. Although the implemented RT-EMS could be executed every $5 \mathrm{~min}$ and still presents better results than previous works, it is executed every $15 \mathrm{~min}$. The main reason is that the system has the MGS, which is continually checking for high load variation and in this case, launches a new optimization cycle. This way, system resources are used optimally. The MG understudy has an RS232 serial connection network for the control of the testbed components and the SCADA 
system, as illustrated in Figure 7.5. The latency time for sending data from the LabVIEW SCADA system to the generators and controllable loads in the testbed is $1 \mathrm{~s}$ [256].

Furthermore, for a measurement message size of 32 bytes and a message rate of 1000 $\mathrm{Msg} / \mathrm{s}$, the average latency for DDS middleware is $243 \mu \mathrm{s}$ with $90 \%$ below $269 \mu$ s [257]. The T-Cell optimization algorithm executed in a computer of 4GB of RAM and $2 \mathrm{GHz}$ of CPU has an average latency of $231 \mathrm{~ms}$. Therefore, the total latency of the RT-EMS is 1.3 $\mathrm{s}$ on average, which is way less than the 3 min presented in [221].

Another positive point could be stated. As the MG size increases and additional components are connected, the system variables will increase. This would be reflected in the execution time that would increase too. However, the proposed immune algorithm was tested along with fast, chaotic particle swarm optimization (PSO) and independent component analysis based PSO in the case of the small and medium power network, and the T-Cell algorithm reached a better optimum in minimum time [226].

\subsection{Discussion}

The proposed RT-EMS has several features that make it easy to implement in MGs as well as in smart buildings. The MAS makes it easier to extend the system and add other components. New agents can be designed for new components, integrated into the agent platform, and then added to the optimization system. Also, the DDS makes it very easy to integrate any hardware to the existing MG. For instance, a new PV system in a smart building can be interfaced with the SCADA using a new publisher and a new topic for this new PV system. Thus, the RT-EMS is modular and easy to extend for larger MGs. 
Nevertheless, the system can be improved by considering the distribution losses and constraints in the optimization. Since the present work focuses on small scale MGs, e.g., smart home, where all generations and consumption units are in the same location, the losses are not significant; for this reason, this assumption was made. However, one of the future improvements of the present work is to consider it. The authors considered the water heater as a controllable load as it can be quickly turn on and off without affecting customer comfort. Besides, the relatively small size of the controllable power used in this work makes it comparable to a water heater. However, in a large $\mathrm{Mg}$, the controllable loads could be considered as the centralized air conditioner, the washing machine or dishwasher that presents a more significant power to control. A second optimization layer between MGOs that negotiate the best option for buying or selling the power depending on the market prices could be added. Besides, the grid-connected mode was considered in this study. Future works can consider the islanded mode of operation that updates the RT-EMS parameters accordingly

\subsection{Conclusions}

Smart cities are no longer a future vision but a reality. Technologies are not only shaping our modern life but also helping us to preserve the environment and adopt a more sustainable life. Several cities around the world launch programs are aiming to preserve the environment. While some cities choose to promote the use of applications to ease the city traffic and therefore reduce gas emissions, others present incentives to its citizens to produce renewable energy and reduce their consumption during peak hours; In this context,

developing a comprehensive EMS that would enhance cities sustainability agenda is a hot 
topic. Our proposed solution that incorporates fast real-time optimization would be a good asset for sustainable cities. A multiagent RT-EMS based on the T-Cell algorithm was implemented and successfully experimented in the smart grid testbed at Florida International University for optimally managing an MG. Three scenarios were tested to show the effectiveness and optimal operation of the MG using the proposed technique. Comparison of simulations and experimental results show the ability of the proposed multiagent T-Cell based RT-EMS in maintaining the stability and smooth operation of the MG. Besides, the modularity and fault tolerance features were more comfortable to implement through the MAS JADE platform. The DDS middleware also allows the interoperability between different components of the system. The results obtained by the multiagent RT-EMS highlight the effectiveness of the proposed algorithm and demonstrate a faster convergence time compared to previous works. The optimum values were obtained faster in terms of computation time as compared to existing techniques. The latency from the proposed system was $43 \%$ faster than other heuristic or deterministic methods in the literature. This significant improvement makes this proposed system more competitive for RT applications. Therefore, our proposed EMS could be envisioned as a real solution for future RT operating MG.

Furthermore, this EMS could also be implemented in smart homes or smart buildings that integrate RE and need RT EMS. Through the use of RT-EMS, the MG operator not only guarantees a reduction of energy consumption cost but also assures the maximum utilization of available RE. As a consequence, the system is more resilient and participate 
in reducing the negative environmental impact. The RT-EMS also applies DR when needed, which has a positive impact on the distribution system during peak hours. 


\section{Chapter 8 Medium Voltage DC Testbed: An Essential Tool to Facilitate the Futuristic integration of DC Microgrids/Nanogrids}

The futuristic distribution system, which is one of the basic smart grid concepts, can be viewed as a cluster of microgrids/nanogrids composed of a cluster of distributed energy resources (DER). Also, the exponential development of solid-state transformers technology which makes it possible to directly integrate the microgrids/nanogrids to the medium voltage (MV) part of the power grid. The MVDC network facilitates the integration of DC microgrids/nanogrids clusters to the AC grid. However, this necessitates the research institutions to develop laboratory equipment to validate the hardware developed to work on the MV levels. This work presents a design of a scaled-down MV DC laboratory testbed. The proposed system is based on Power Electronic Transformers (PETs) in order to provide the high step-up ratio of voltage without occupying much space. This MV DC testbed is a laboratory setup that provides a power source and a power sink for any DC hardware to be tested in the MV range. The proposed system is evaluated through Matlab Simulink simulations.

\subsection{Introduction}

The microgrids/nanogrids offer an essential solution to enable a resilient grid infrastructure since they have the ability to continue operating in case of a utility outage [258]. The futuristic distribution system that is one of the primary smart grid concepts can be viewed as a cluster of microgrids/nanogrids [259] and each microgrid/nanogrid can be viewed as a cluster of distributed energy resources DERs, which has a connection to the 
primary utility grid. Microgrids could be composed of conventional or renewable DERs. Microgrids mostly depend on intermittent renewable resources. This configuration may introduce severe stability problems, especially during the islanded operation [260], [261]. Minor stability problems can cause cascading outages that would result in a large-scale blackout if proper action is not taken on time. An efficient way to prevent such risks, ensure robustness, and resilience is to operate the grid in a coordinated mode [262], [263] with considering the different parameters of the heterogeneous mix of the available resources. The microgrids are categorized as AC and DC. There is a trend to consider DC microgrids widely. The reasons for this are well discussed in the literature [264]-[270]. On the other side, the advantages of $\mathrm{AC}$ systems cannot be omitted; this is mainly due to the efficient and cheap means of generation provided by the synchronous AC machines. The majority of the loads have been designed to operate in AC form [268]. Thus, a combination of AC and DC microgrids can be introduced as a viable solution. Under the smart grid views, these microgrids/nanogrids are mostly a distributed DC grids. Therefore, The MVDC network facilitates the integration of DC microgrids/nanogrids clusters to the AC grid. The MVDC network has introduced before for MV DC shipboard power systems and electrified transportation means charging stations are attracting many research interests in the past few years [271], [272]. Providing a realistic laboratory-based environment for testing these MV DC power electronic systems is a challenging problem since it requires a high voltage power $(>1 \mathrm{kV})$ and a high voltage dc electronic load.

The exponential development in power switching devices by introducing the high power wide-band-gap devices that are capable of switching at high frequencies [273]-[275], and the availability of advanced magnetic materials like amorphous and monocrystalline which 
have excellent magnetic characteristics for medium and high-frequency applications, such as high permeability, high saturation flux density, and relatively low core losses [276], have brought an extensive attention to solid-state transformers (SST) [277]. SST is a medium/ high-frequency transformer preceded and followed by two power converters, one of them provides the high frequency (HF) input voltage to the primary side of the transformer, and the other power converter (following the transformer) converts the HF stepped up/down voltage from the secondary side of the transformer to the desired voltage profile (AC at the nominal frequency or DC). The SST operates at medium/high frequencies which help explicitly in reducing its volume and weight. Reliable state transformers can have a single core as in [278] or multiple cores as in [279]. The development of the SST has influenced and attracted a lot of research in the area of integrating medium size microgrids directly to the MV part of the power grid using HF medium voltage power electronic interfacing systems without the need to the bulky and heavy low-frequency power transformers [280]. This growing development of this kind of MV power electronic systems requires the research institutions to develop tools and testbeds to test and validate the developed hardware.

In this work, an architecture of MV DC testbed based on power electronic transformers (PETs) to have a high step-up gain or high step down gain. The PET is a medium/ highfrequency transformer preceded and followed by two power converters, one of them provides the high frequency (HF) input voltage to the primary side of the transformer, and the other power converter (following the transformer) converts the HF stepped up/down voltage from the secondary side of the transformer to the desired voltage profile (AC at the nominal frequency or DC) [281]-[284]. The Construction of the proposed MV DC testbed 
is discussed in section II, section III presents the control scheme of the system, preliminary results are shown in section IV, and finally, the conclusion is given in section V.

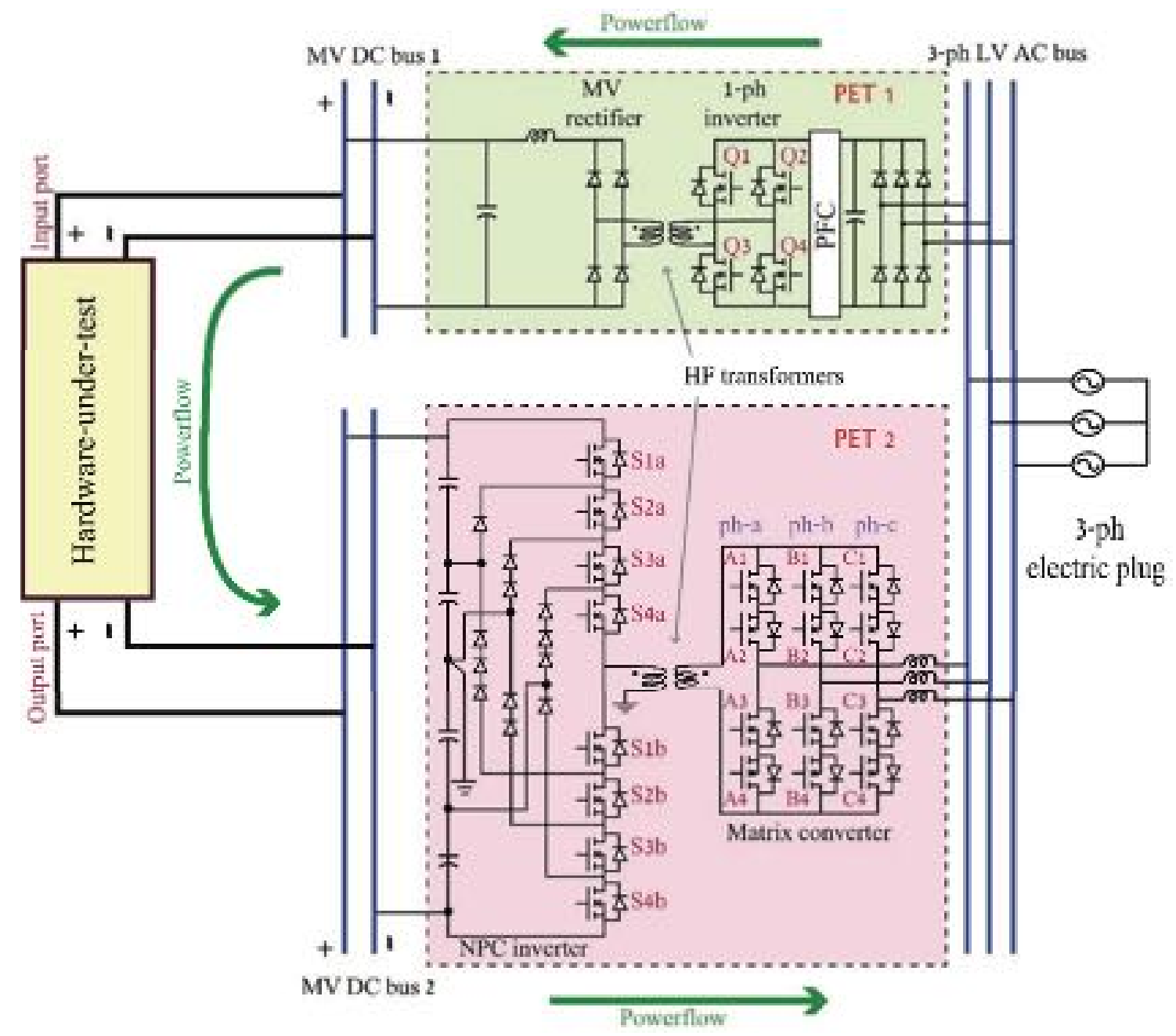

Figure 8.1 The architecture of the proposed MV DC testbed.

\subsection{Construction of PET I}

PET I is depicted in Fig. 8.1; it comprises four stages. The first stage is an LV threephase uncontrolled rectifier to rectify the three-phase $\mathrm{AC}$ voltage coming from the electric plug. The second stage is a full-bridge inverter to convert the DC link voltage to an HF square wave voltage required by the HF transformer. The third stage is an HF step-up 
transformer to amplify the square wave voltage to the required voltage level (square wave pulses swinging between $5 \mathrm{KV}$ and $-5 \mathrm{KV}$ ). The fourth stage is an MV uncontrolled rectifier to rectify the square wave voltage and provides the medium voltage DC voltage of the MVDC bus; the MV rectifier relies on the DC link capacitors of the neutral-point-clamped (NPC) inverter in SST II instead of having its own DC link capacitors. SSI is an uncontrolled unidirectional step-up power electronic converter, and the HF transformer turns ratio is the only means to control the MVDC bus voltage magnitude (in the design phase not in the operational phase). The MVDC voltage level can be manipulated by applying the phase-shift control technique described in [285], [286], and an LC filter after the MV rectifier will be required in this case.

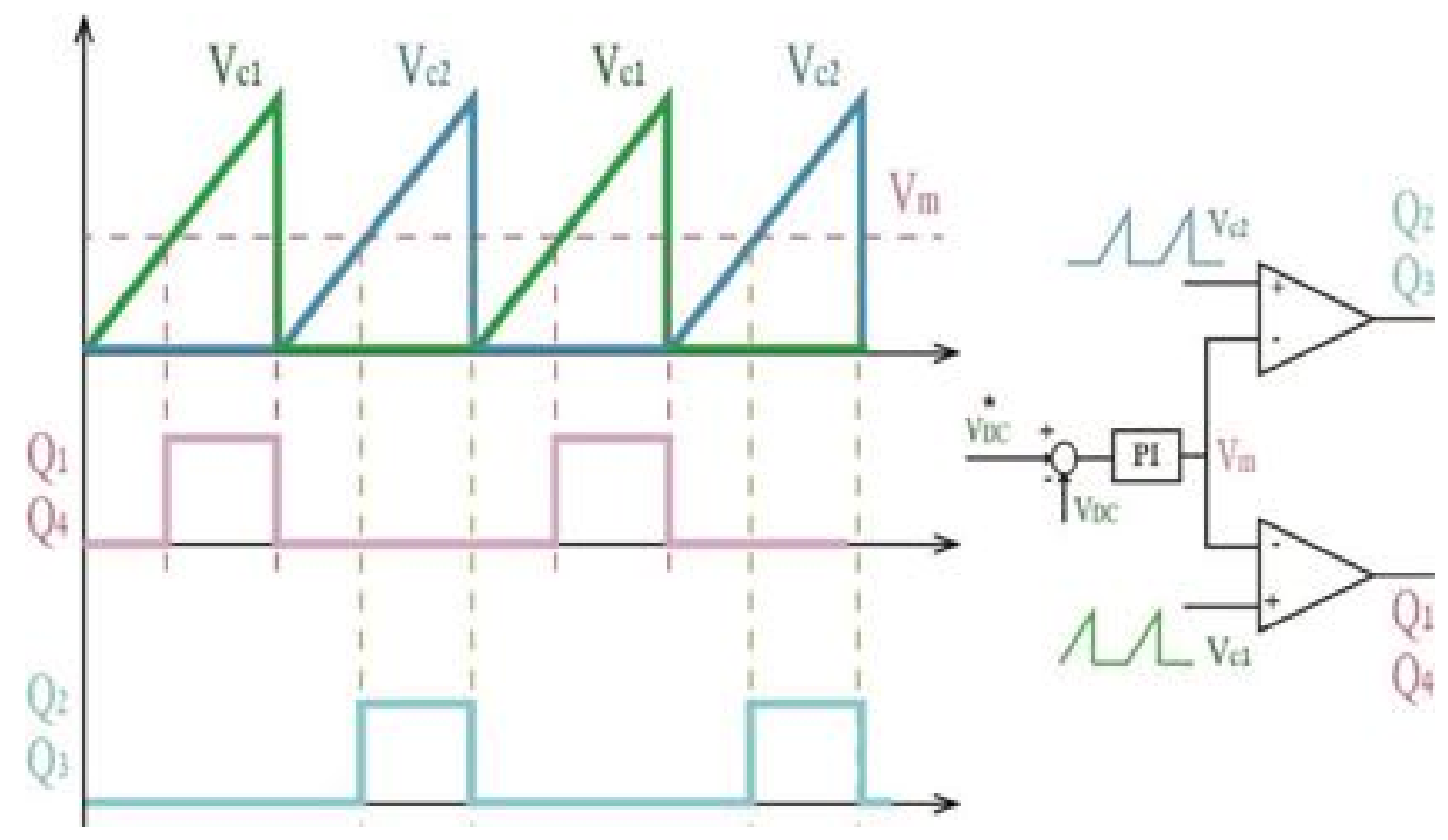

Figure 8.2 Controller of the single-phase inverter of PET1.

The primary design methodology of the proposed MVDC testbed is to keep the size and volume as small and compact as possible, so the proposed design of PET I gave up the 
voltage magnitude controllability feature of the MVDC bus to get rid of the extra LC filter following the MV rectifier, thus instead, the voltage magnitude of the MVDC bus is set at $5 \mathrm{KV}$, which is appropriate to test most of the MV power devices and systems. A higher voltage level requires a higher number of levels of the NPC inverter in PET II (more power switches) and larger transformers. The control schemes of the single-phase inverter of PET1 is shown in Fig. 8.2.

\subsection{Construction and Control of PET II}

The structure of PET II is shown in Fig. 8.1; it consists of three main stages. The operation and function of each stage are discussed and illustrated as follows.

\subsubsection{Five-level Neutral-Point-Clamped Square-wave Inverter}

The neutral-point-clamped (NPC) inverter is the first stage after the MVDC bus, and its main function is to convert the MVDC voltage on the MVDC bus to an HF square-wave voltage required by the HF transformer. Detailed illustration of the operation of the NPC inverter is in [287]. The MVDC link voltage is divided over four capacitors; the voltage over each of them equals $V_{D C} / 4(=1.25 \mathrm{KV})$. The structure of the leg of the NPC inverter resembles the structure of the leg of the LV inverter with the replacement of each power switch by four power switches. A path for the load current in the positive and negative direction should be provided for each voltage level with the help of the voltage clamping diode. The MVDC link voltage is applied sequentially to the primary side of the transformer. The switching sequence associated with each voltage level is shown in Fig.8.3. $\mathrm{T}_{\text {pulse }}$ is the time of $\mathrm{V}_{\mathrm{DC}} / 2$ or $-\mathrm{V}_{\mathrm{DC}} / 2$ realization, and $\mathrm{T}_{\text {step }}$ is the time that separates between two consecutive levels, and it is minimal (in the range of tens to hundreds of 
Nanoseconds) compared to $\mathrm{T}_{\text {pulse. }}$ The square-wave voltage with fine stepping requires a dedicated circuit to provide the gate pulses for the eight power switches of the NPC inverter.

A firing circuit is proposed in Fig. 8.4; the circuit provides the fine stepping by means of monostable timers. The firing circuit is fed with reference square waveform $(\mathrm{P})$; negative and positive edge detectors fire short pulses at each edge of $\mathrm{P}$; these short pulses are then supplied to monostable timers. $\mathrm{V}_{\mathrm{DC}} / 2$ and $-\mathrm{V}_{\mathrm{DC}} / 2$ have two designated monostable timers, one of them fires a high pulse at the edge of $\mathrm{P}$ with a pulse width of $\left(2 \mathrm{~T}_{\text {step }}+\mathrm{T}_{\text {pulse }}\right)$, and the other one fires a low pulse at the edge of $\mathrm{P}$ with a pulse width of $\left(2 \mathrm{~T}_{\text {step }}\right)$. Passing the output of the two monostable timers through an AND gate produces the reference pulse width of $\mathrm{V}_{D C} / 2$ or $-\mathrm{V}_{\mathrm{DC}} / 2$. A demultiplexer is used to select whether the reference pulse generated is for $\mathrm{V}_{\mathrm{DC}} / 2$ or $-\mathrm{V}_{\mathrm{DC}} / 2$. The same technique is used to generate the reference pulses of $\mathrm{V}_{\mathrm{DC}} / 4$ or $-\mathrm{V}_{\mathrm{DC}} / 4$. There are another two monostable timers designated for these voltage levels, one of them fires a high pulse at the edge of $\mathrm{P}$ with a pulse width of $\left(3 \mathrm{~T}_{\text {step }}+\mathrm{T}_{\text {pulse }}\right)$ and the other timer fires a low pulse at the edge of $\mathrm{P}$ with a pulse width of $\left(\mathrm{T}_{\text {step }}\right)$. Passing the output of both timers through an AND gate generates the reference pulses for $\mathrm{V}_{\mathrm{DC}} / 4$ or $-\mathrm{V}_{\mathrm{DC}} / 4$; a demultiplexer selects whether the generated pulses to be for $-\mathrm{V}_{\mathrm{DC}} / 4$ reference or $\mathrm{V}_{\mathrm{DC}} / 4$ reference. The reference for $(0 \mathrm{v})$ voltage level exists whenever the reference of $-\mathrm{V}_{\mathrm{DC}} / 2, \mathrm{~V}_{\mathrm{DC}} / 2,-\mathrm{V}_{\mathrm{DC}} / 4$, or $\mathrm{V}_{\mathrm{DC}} / 4$ does not exist.

Combinational logic circuits, as shown in Fig. 8.4 use the reference pulses for each level of the NPC inverter to trigger the power switches associated with each voltage level. 
The buffer circuit has two functions; the first one is to provide galvanic isolation between the control circuit and the power switches; the second function if to translate the triggering pulses of the control circuit to the adequate voltage level required by the power switches. An HF transformer follows the NPC inverter to step down the voltage level of the voltage pulses generated by the NPC inverter.

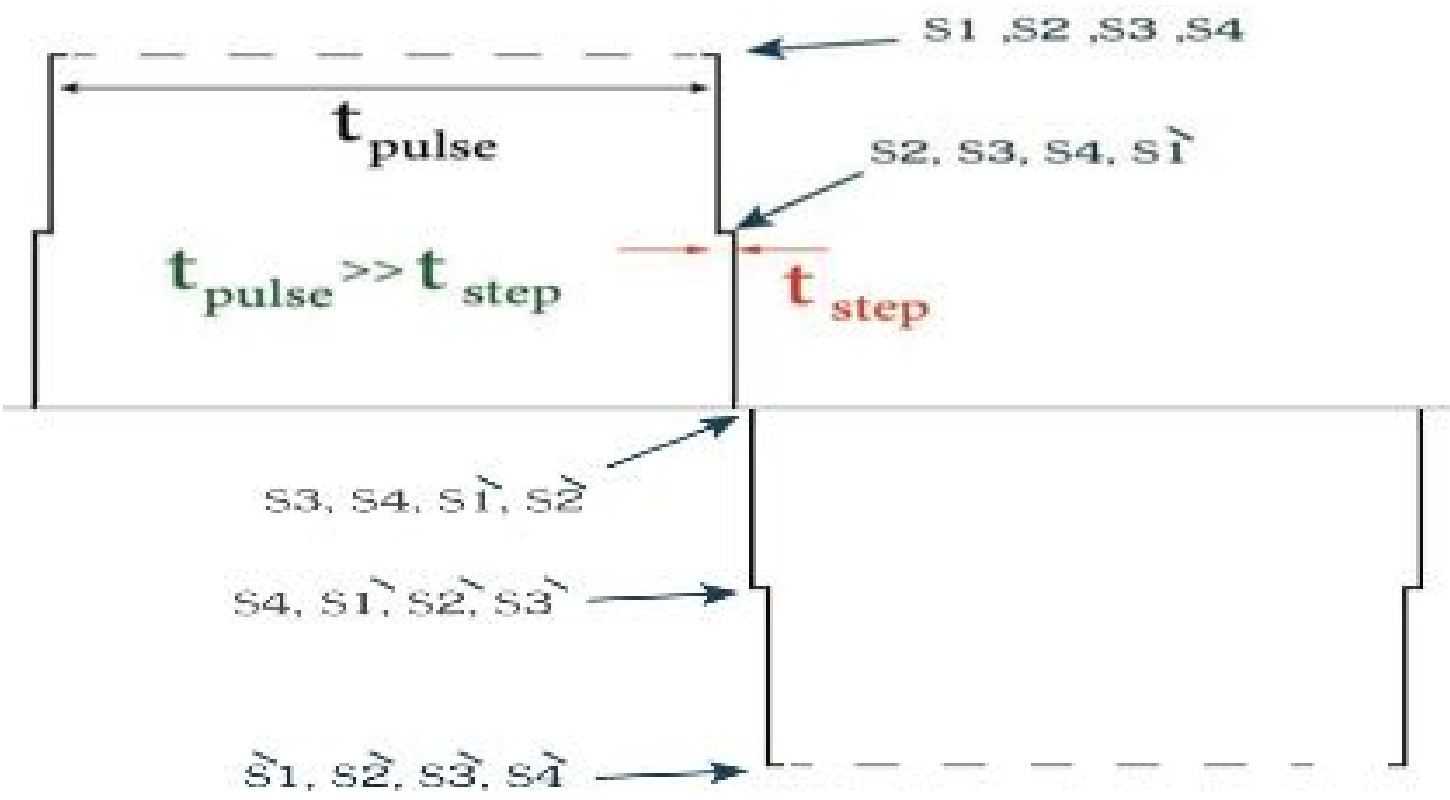

Figure 8.3 Switching sequence associated with each voltage level of the 5-level NPC inverter

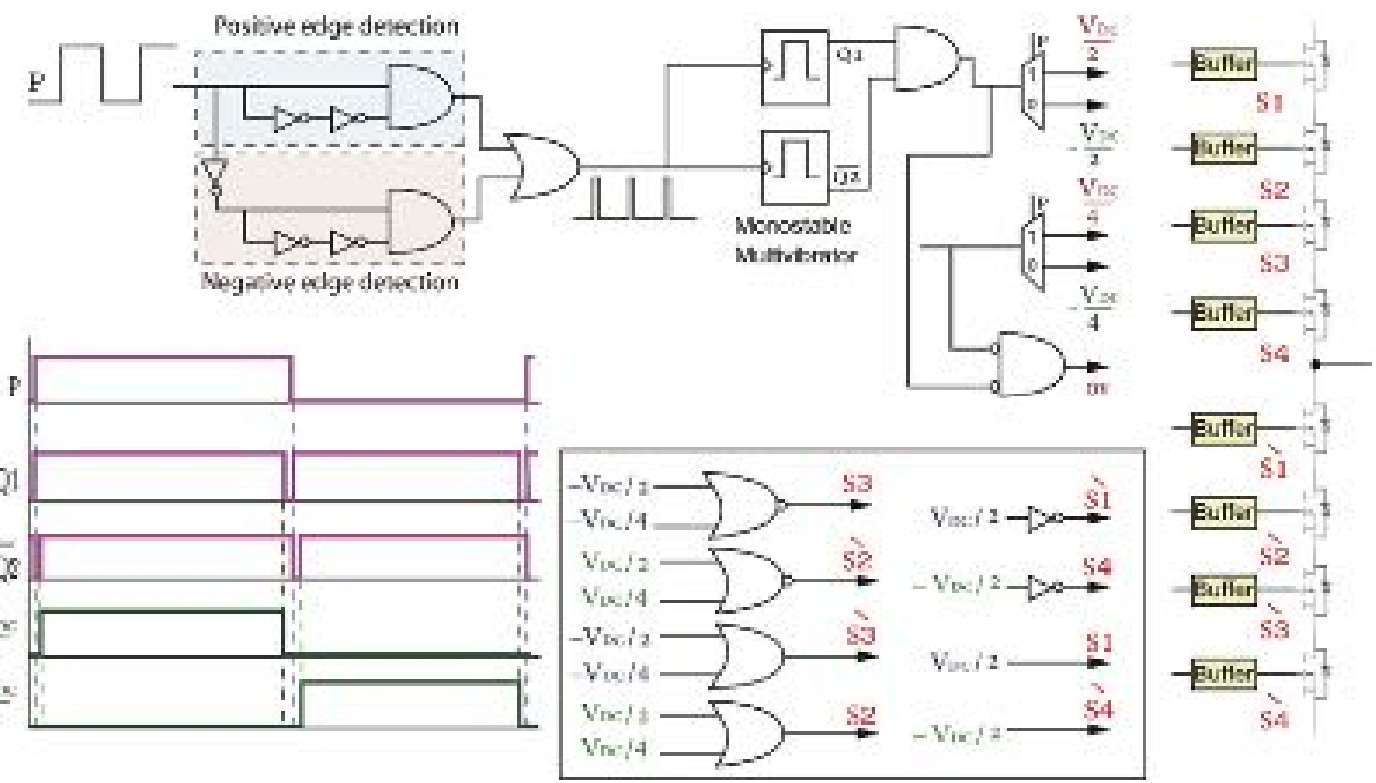

Figure 8.4 The proposed firing circuit of the 5-level NPC inverter 


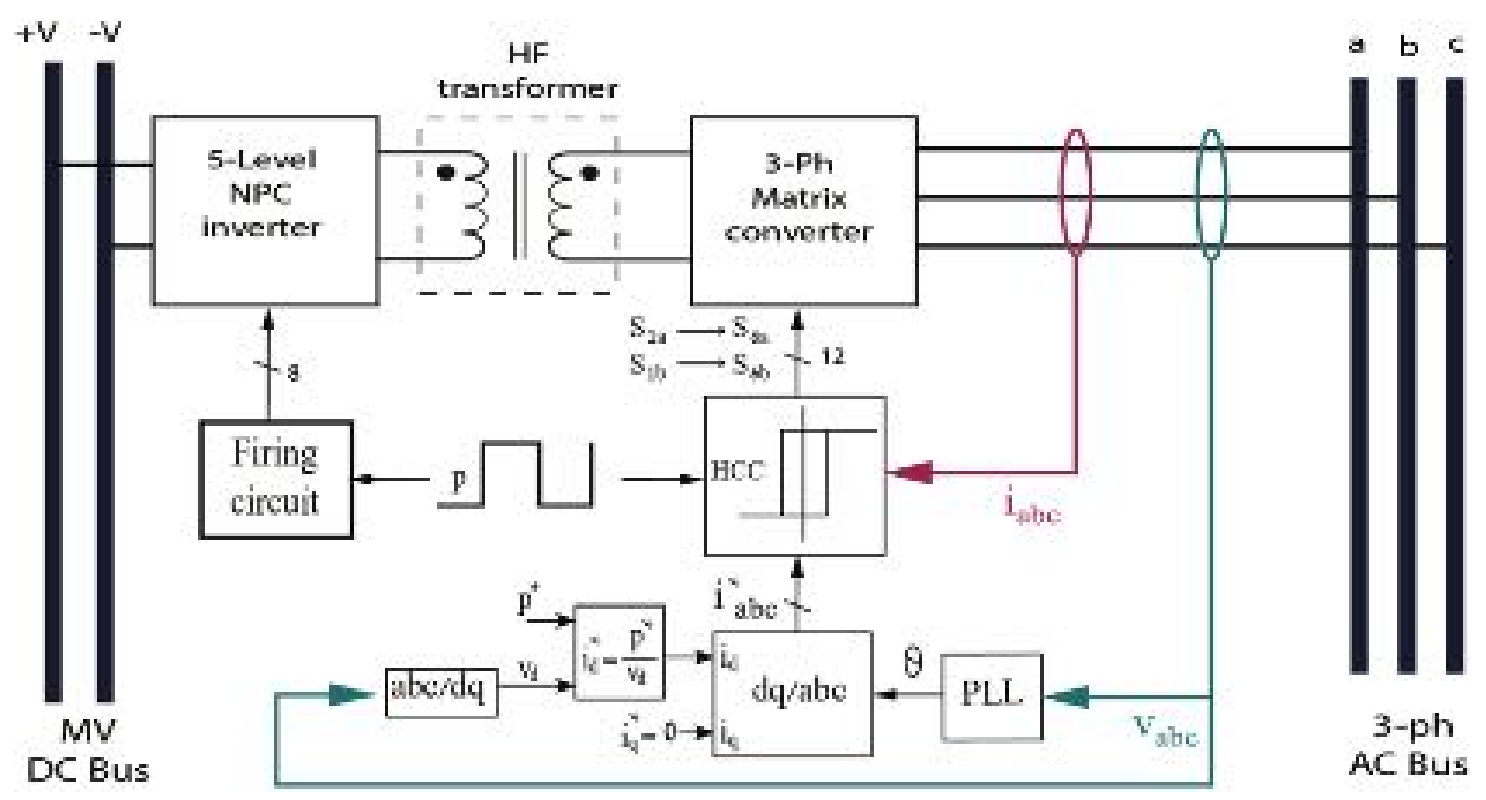

Figure 8.5 The proposed firing circuit of the 5-level NPC inverter

\subsubsection{Three-phase matrix Converter}

A matrix converter (MC) follows the HF transformer (instead of a back-to-back converter, to eliminate the need for DC link capacitors) to convert the HF square-wave voltage to three-phase voltage at the grid voltage level. The voltage at the point of common coupling (PCC) with the three-phase bus system is measured and passed to a phase-lockedloop (PLL) circuit to calculate the phase angle of the grid voltage $(\theta)$. A hysteresis current controller (HCC) is used to generate the switching signals to the power switches of the MC [280]. HCC is an easy technique to directly control the current, where it forces the current vector supplied from the MC to track a reference trajectory. If the actual current exceeds the reference current plus the positive tolerance band error, the HCC output goes to zero, and if the actual current is lower than the reference current plus the negative tolerance band error, the output goes to one. For the $\mathrm{MC}$, the implementation of the $\mathrm{HCC}$ requires the knowledge of the HCC output, the polarity of the reference current, and the polarity of the input voltage to the $\mathrm{MC}$. The polarity of the input voltage to the $\mathrm{MC}$ converter can be 
acquired from the driving pulses of the NPC inverter $(\mathrm{P})$, where the voltage is positive when $\mathrm{P}$ is one (high) and negative when $\mathrm{P}$ is zero (low). The HCC switching sequence of leg 'a' of the matrix converter is shown in Table 8.1

Table 8.1 The HCC Switching Sequence of leg 'a' of the MC

\begin{tabular}{|c|c|c|c|c|c|c|}
\hline \multirow{2}{*}{ HCC Output } & \multirow{2}{*}{$\mathrm{P}$} & \multirow{2}{*}{$\begin{array}{l}\text { Polarity of } \\
\text { the Reference Current } \mathrm{I}_{\mathrm{a}}{ }^{*}\end{array}$} & \multicolumn{4}{|c|}{$\begin{array}{l}\text { Switches } \\
\text { Status }\end{array}$} \\
\hline & & & $\mathrm{S}_{\mathrm{la}}$ & $\mathrm{S}_{4 \mathrm{a}}$ & $\mathrm{S}_{4 \mathrm{~b}}$ & $\mathrm{~S}_{\mathrm{lb}}$ \\
\hline 1 & 1 & Positive & 1 & 0 & 0 & 0 \\
\hline 1 & 1 & Negative & 0 & 0 & 1 & 0 \\
\hline 1 & 0 & Positive & 0 & 0 & 0 & 1 \\
\hline 1 & 0 & Negative & 0 & 1 & 0 & 0 \\
\hline 0 & 1 & Positive & 0 & 0 & 0 & 1 \\
\hline 0 & 1 & Negative & 0 & 1 & 0 & 0 \\
\hline 0 & 0 & Positive & 1 & 0 & 0 & 0 \\
\hline 0 & 0 & Negative & 0 & 0 & 1 & 0 \\
\hline
\end{tabular}

\subsubsection{The programmable load operation of PET II}

Vector control is utilized to decouple between the active and reactive power components (P and Q) [288]. In Fig. 8.6, the reference to the quadrature current component $\left(\mathrm{i}^{*} \mathrm{q}\right)$ of the injected current to the three-phase AC bus is set to zero (no reactive power is injected into the three-phase AC bus). The reference of the direct current component $\left(i^{*} d\right)$ is calculated directly from the reference power $\left(\mathrm{p}^{*}\right)$ given by the user, and the direct voltage component of the three-phase AC bus voltage $\left(V_{d}\right)$. After calculating the reference $d-q$ currents, inverse Clark and inverse Park transformations are utilized to calculate the reference three-phase current $\left(i^{*}\right.$ abc $)$ from $i^{*}{ }_{d}$ and $i^{*}$ q. 


\subsection{Simulation Results}

The model of the proposed MV DC testbed is simulated in Matlab Simulink, and the parameters of the model are shown in Table 8.2

Table 8.2 MVDC simulation model parameter

\begin{tabular}{|c|c|c|c|}
\hline \multicolumn{4}{|c|}{ Simulated system parameters } \\
\hline \multicolumn{4}{|c|}{ PET1 parameters } \\
\hline $\mathrm{L}$ & $680 \mu \mathrm{H}$ & $\mathrm{C}$ & $10 \mu \mathrm{F}$ \\
\hline \multicolumn{2}{|c|}{ HF transformer frequency } & \multicolumn{2}{|l|}{$2 \mathrm{kHz}$} \\
\hline \multicolumn{2}{|c|}{ Carrier frequency } & \multicolumn{2}{|l|}{$30 \mathrm{kHz}$} \\
\hline \multicolumn{4}{|c|}{ PET2 parameters } \\
\hline \multicolumn{2}{|c|}{ HF transformer frequency } & \multicolumn{2}{|l|}{$2 \mathrm{kHz}$} \\
\hline \multicolumn{2}{|c|}{ Matrix converter frequency } & \multicolumn{2}{|l|}{ variable } \\
\hline \multicolumn{2}{|l|}{$\mathrm{L}$} & \multicolumn{2}{|l|}{$2 \mathrm{mH}$} \\
\hline
\end{tabular}

Both Figures 8.6 and 8.7 are dedicated to showing the performance of PET1 under different reference voltage levels of MV DC bus 1, where Fig. 8.6 shows that the actual

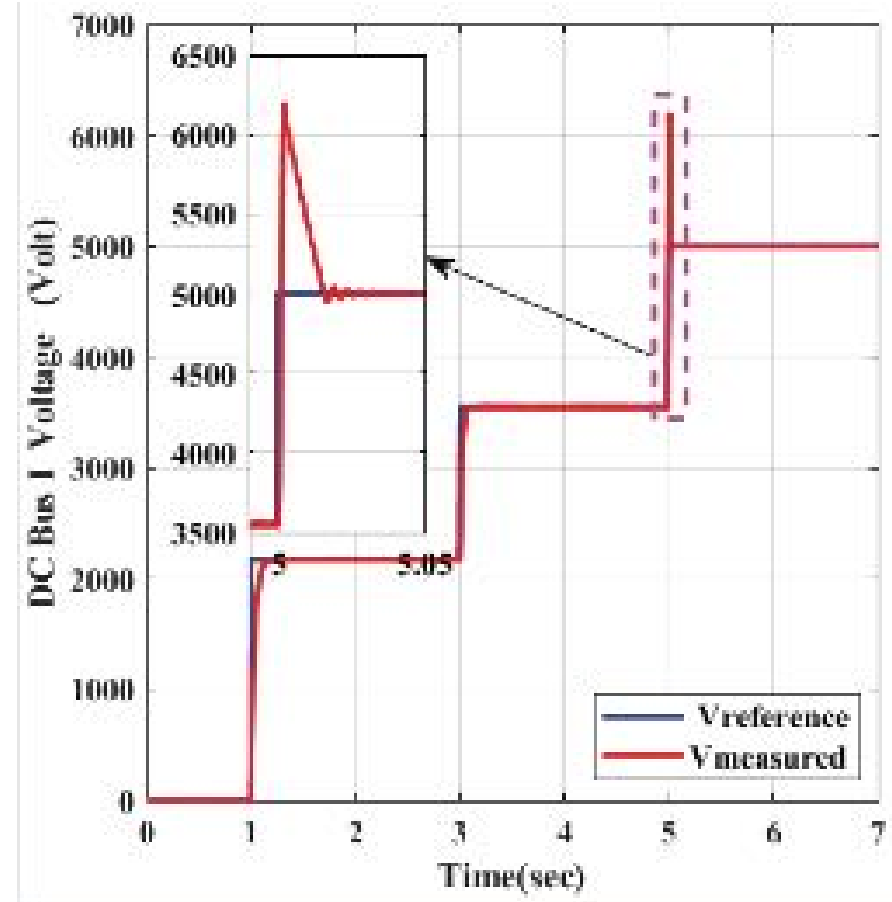

Figure 8.6 MV DC bus 1 voltage with different reference levels 
voltage is tracking well the reference voltage. Specifically, the control algorithm shows robustness and firmness in changing the voltage levels across different steps of the grid operation without encountering any operational deficiencies. As an example, figure 8.6 shows the voltage level being stepped-up from zero to $2.3 \mathrm{Kv}$ without the occurrence of any regular voltage issues that occur in such processes, such as voltage overshoots or oscillations. This is true and could be noticed even in the case of voltage changes from 2.3 Kv to $3.5 \mathrm{Kv}$, respectively. Fig. 8.7 shows the pulses used to trigger the four switches of the single-phase inverter of PET1. In this case scenario, we decide to test the converters under fixed voltage conditions with variable power reference this time, comparing to variable voltage levels at fixed power reference in the previous case scenario. As we can see from figure 8.7, the output power values followed the referenced power values at a robust and accurate fixed voltage level. This is a valid indication of the accuracy and validity of our proposed control methodology.
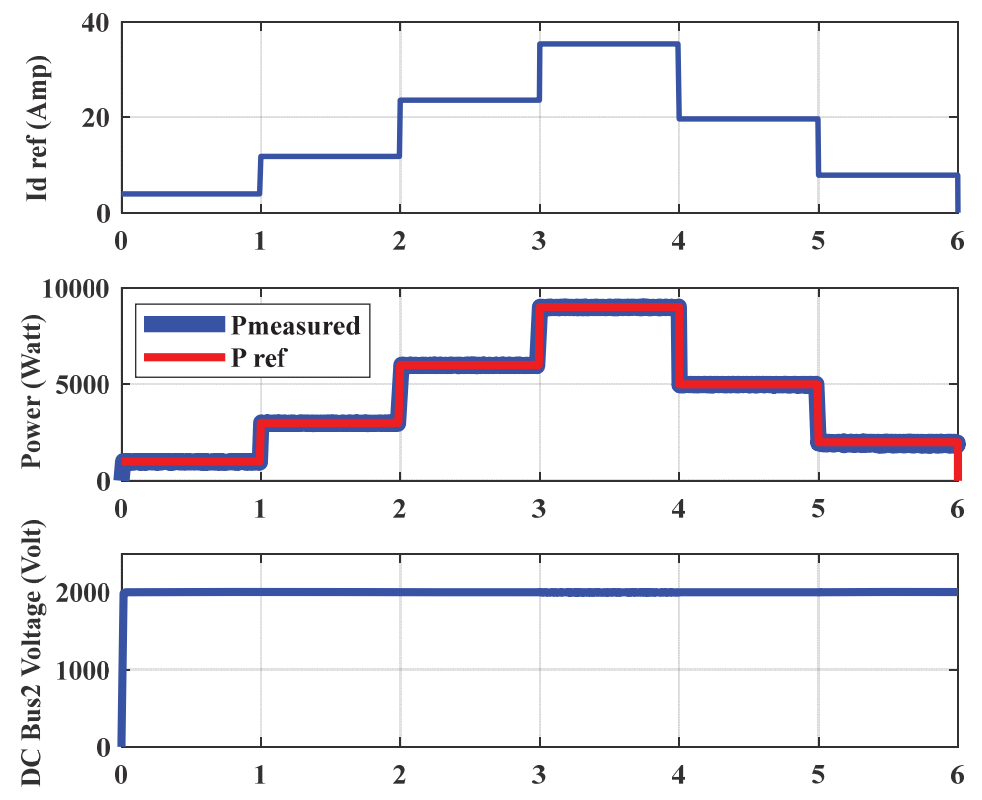

Figure 8.7 Injected power from PET2 to the grid (different power levels at a fixed voltage level at MV DC bus2). 


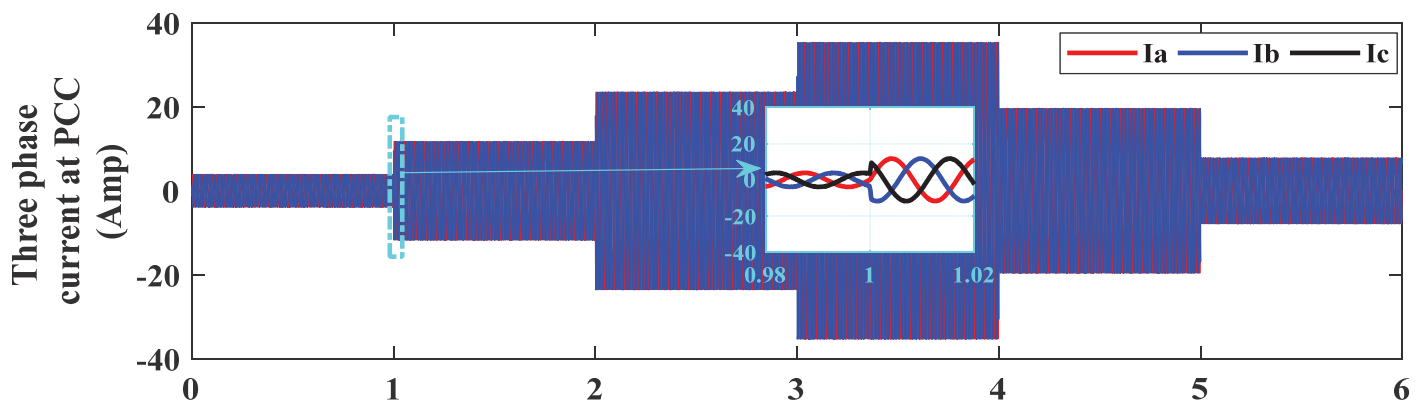

Figure 8.8 Three-phase currents injected from PET2 to the grid at different power levels

Figures 8.7 and 8.8 show the performance of PET 2 (under varying power levels and fixed voltage of MV DC bus 2) and (under a fixed reference power and varying voltage level of the MV DC bus 2), respectively. Fig. 8.8 shows the three-phase grid currents injected by PET2 to the grid under different reference power levels.

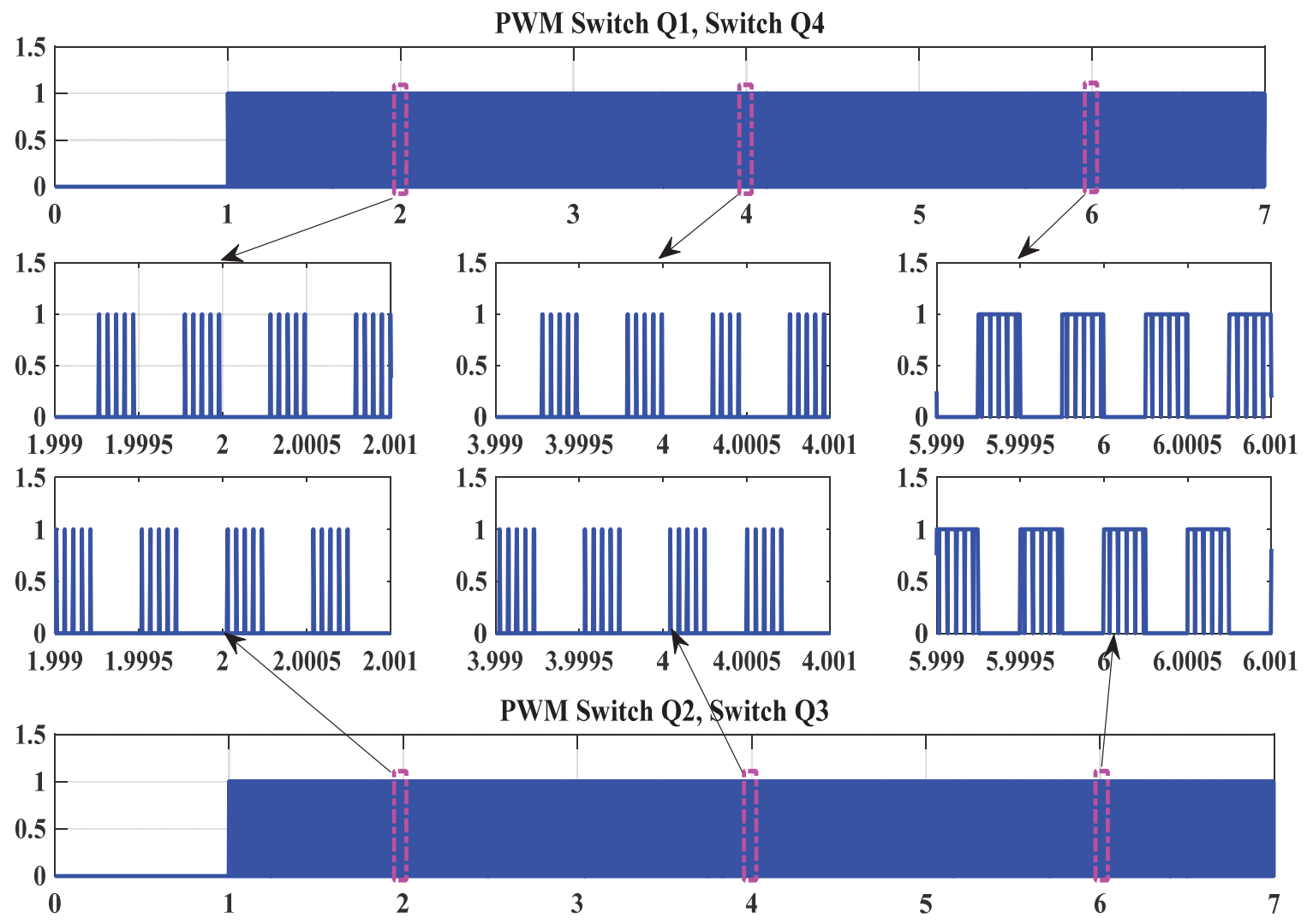

Figure 8.9 Switching pulses of the four switches of the single-phase inverter of PET1 
Figure 8.9 presents the results of the PWM driving signals for all the four switches of the inverter PETI. Specifically, the figure illustrates zoomed screenshots of both upper and lower switches pulses for the purpose of more clarification. As someone may notice, both the upper and lower pulses complement each other with the width of PWM changes periodically due to the changes in power levels. Figure 8.10 shows different corresponding power levels measured at a fixed voltage level of the MV DC part. To be specific, we fixed the power at the point of the coupling and applied variable voltage levels considering different case scenarios at the DC bus in order to test the capability of our proposed scaleddown MV DC testbed. Furthermore, the results show that voltage levels were changing safely between different operational levels (e.g., from 2, 2.5, 3, and $4 \mathrm{kV}$ ) without leading to any power oscillations problems, as can be concluded from the power levels shown in figure 8.10.

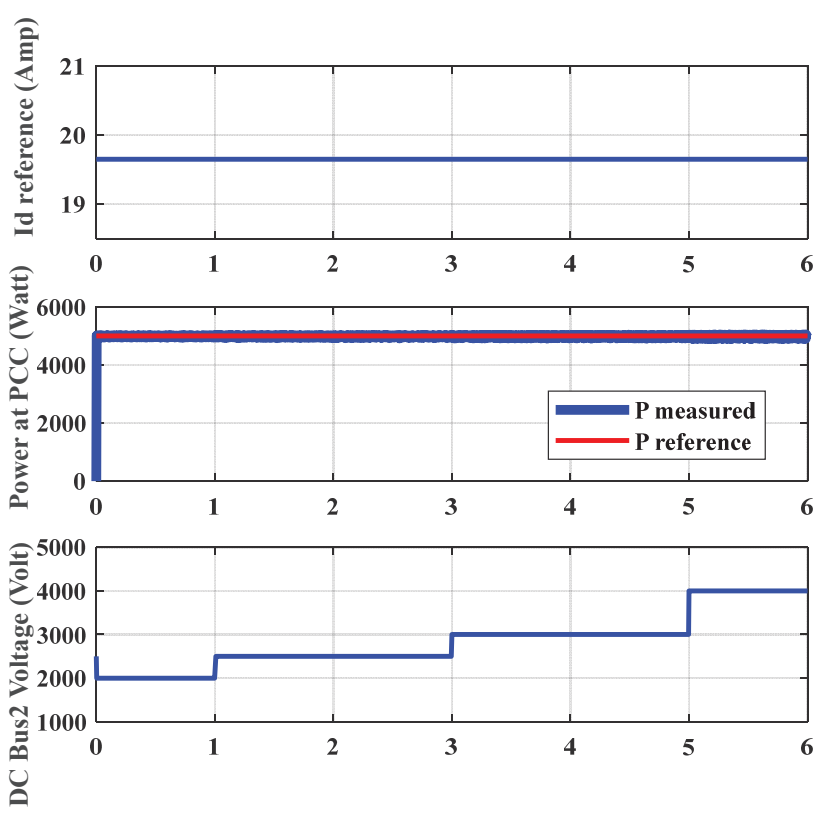

Figure 8.10 Injected power from PET2 to the grid (different power levels at a fixed voltage level at MV DC bus2). 


\subsection{Conclusion}

This paper presented a detailed architecture of an MV DC testbed that is developed based on the concept of power electronic transformers. Specifically, the circuit diagrams of the two power electronic transformers and their associated controls are documented throughout the testing procedure. Preliminary results indicate the behavior of the proposed system under severe steady-state and transient conditions. 


\section{Chapter 9 Fault Detection and Compensation for a PV System Grid Tie Inverter}

The primary purpose of this work is to propose a fault detection and fault-tolerant control mechanism to maintain the continuous operation of the grid-tied inverter, which is commonly used to interface renewable energy and distributed generation to the grid. The fault tolerance is provided using the modified topology of the DC-AC power converter with a redundant standby branch. The proposed system ensures continuous operation without isolating the energy source from the grid during faults on the inverter switch. The fault may be an open circuit fault or short circuit fault.

\subsection{Introduction}

Increasing demand of electrical energy with the rapid change of climate lead to the intention of maximizing the penetration of renewable energy versus the decrease of using fossil fuels. One of the most promising types used to generate electrical energy from renewable energy is the photovoltaic (PV). There are different topologies used to connect PV energy to the utility grid such as Centralized, String, Multi-string, Module and Modular configuration topology [289]. All of these topologies can be summarized in two different structures, two-stage structure and one stage structure in which the primary power electronics device used to connect such a system to the utility grid is the inverter. It is essential to increase the reliability and survivability of the inverter by increasing the inverter fault tolerance to ensure continuous feeding utility by this type of distributed generation (DG) energy. In the last three decades, there are many literatures on the fault tolerance of inverter with drive system such as studies focused on the fault-tolerant control of inverter feeding passive load, some of them focusing on fault detection [290]-[291], 
fault detection and identification [292] and the other present integration of fault detection and identification and compensation in one topology [293]. The main causes of inverter malfunction are the DC bus capacitor and semiconductor failures. The semiconductor switch failures can be classified into open circuit faults and short circuit faults. This work presents a fault detection control algorithm used to detect, isolate, identify fault occurs on one of the inverter switches and reconfigures the inverter circuit by using modified inverter topology. So its focus on the fault occurs on the semiconductor switches. To avoid a complete loss for one of the inverter branches. The detection mechanism will detect abnormal inverter operation. The identification mechanism will identify the source and location of the faulty component and trigger reconfiguration action. Using such an algorithm to increase the reliability of the grid-tie inverter is a significant issue, especially in the smart grid in which renewable energy sources will supply a high level of energy. These cause a big stability problem in the case of sudden isolation in a significant portion of energy.

\subsection{System description}

A typical grid-connected PV system shown in Figure 9.1, which includes six main parts: PV arrays, a DC-DC Boost converter, DC Bus represented by Capacitor bank, a DCAC converter (proposed inverter topology), LC filter, and a power transformer.

\subsubsection{PV Array}

A typical $60(\mathrm{KW})$ array is constructed from a number of series and parallel connected modules, and this module is a typical Solarex MSX60 PV 60 (W) module constructed from 
36 series Polycrystalline cells [294]. Table 9.1 presents the specification for the PV module at Temperature $250 \mathrm{C}$ and irradiance of $1000 \mathrm{~W} / \mathrm{m} 2$ [294].

\subsubsection{DC-DC converter}

A DC to DC boost converter is inserted at the output of the PV array. Its inductance is $30 \mathrm{mH}$, and capacitance $1000 \mu \mathrm{F}$ and the switch used is IGBT is operating on a $50 \mathrm{kHz}$ switching frequency. This converter has two main targets: first, to step up the output voltage of the PV array to be around the voltage level of the DC Bus. Second, absorb as much as possible from array power by performing maximum power point tracking (MPPT) algorithm to keep track of maximum power with the ambient change, for example, temperature change or irradiance change. This means the converter is working in power control mode and as a result, the DC bus voltage will be continually increased and led to make the voltage of the DC. The bus reaches an unwanted limit which may cause damage, the $\mathrm{DC}$ bus, but the $\mathrm{DC}$ will solve this problem to $\mathrm{AC}$ converter control which illustrated later.

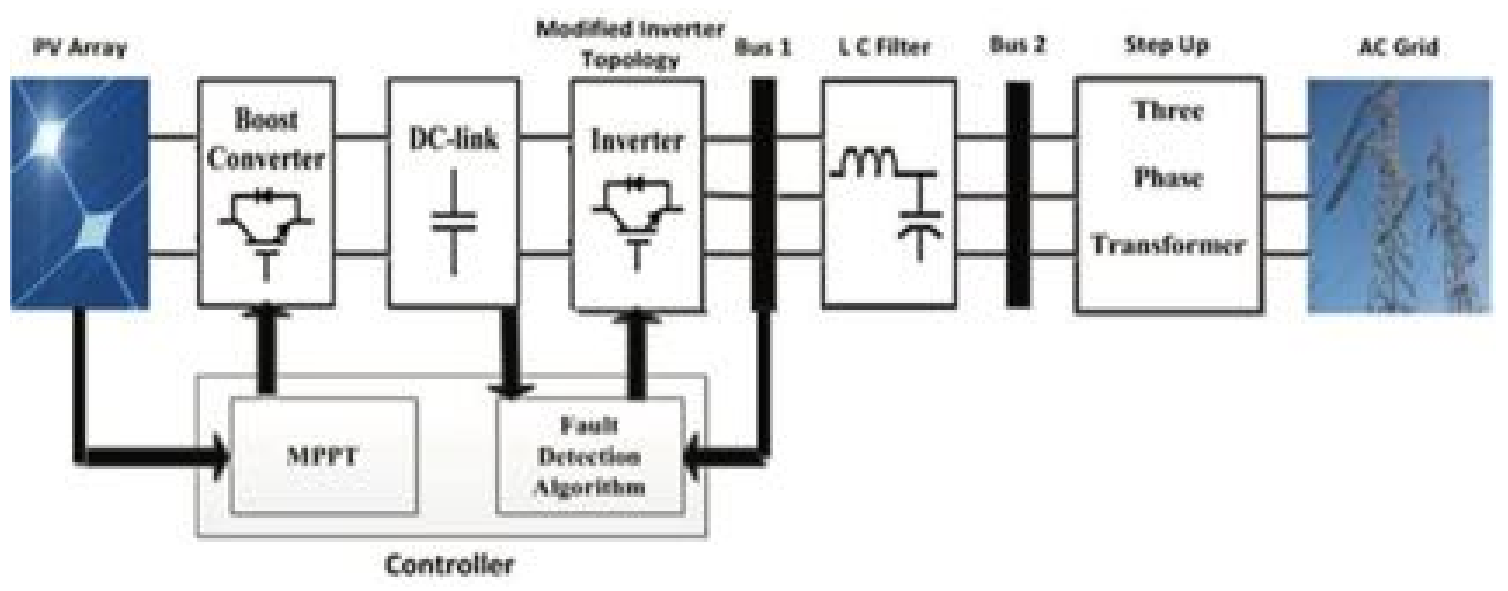

Figure 9.1 An overall grid-connected PV system with modified inverter topology 
Table 9.1 PV MODULE SPECIFICATION

\begin{tabular}{|l|c|}
\hline Open Circuit Voltage (Voc) & 21.0 \\
\hline Short Circuit Current ( Ish ) & 3.74 \\
\hline The voltage at Maximum Power (Vmax) & 17.1 \\
\hline Current at Maximum Power (Imax) & 3.50 \\
\hline Maximum Power (Pmax) & 59.9 \\
\hline
\end{tabular}

\subsubsection{DC bus}

Constructed from the capacitor bank, it is total capacitance value is $2000 \mu \mathrm{F}$ and the rated voltage is $1200 \mathrm{~V}$.

\subsubsection{Proposed DC - AC Converter}

A DC-AC converter (proposed Inverter) is the main part of the Grid Connected PV system, which is commonly used in the Two-stage structure and One stage structure of the PV system. The main target for it is to convert the energy from the DC side of the PV system to the AC side of the utility grid at the same voltage sequence and frequency of the utility grid and is responsible for maintaining the voltage of the DC bus to remain constant. This can be done by regulating the rate of power transfer to the $\mathrm{AC}$ side to be equal the rate of injecting power from the MPPT controller. In this work, a proposed circuit topology for the Inverter is used to reconfigure the inverter circuit after detecting and identify the fault occurrence. This inverter topology depends on using a redundancy branch to substitute the faulty branch after fault occurrence. The reconfiguration process is performed through 
three triacs used as bidirectional power flow switches to connect a redundant branch with the isolated grid phase. Figure 9.2 shows the circuit diagram for the fault-tolerant inverter topology.

\subsection{Fault Detection And Isolation}

For a grid-tied inverter, a fault detection algorithm needs to distinguish between internal and external faults. External faults on the grid can be detected from positive and negative sequence components in the voltage and currents while internal faults can be detected from the DC components resulted from asymmetrical switching activities when a semiconductor switch fails. The magnitude and direction of the DC components in each phase can be used to identify the phase and failure switch.

To isolate the DC component in the inverter output, a low pass filter with a cutoff frequency of $20 \mathrm{~Hz}$ was used to reject the $\mathrm{AC}$ current. One filter was used for each phase. The filtered three-phase current then fed to detection logic, which detects the phase and switch based on the current magnitude and direction in each phase. The fault simulation shows that for an open circuit fault, the phase that has a faulty switch always has the smallest DC components, and the direction of these DC components is related to the switch position in this phase.

For a short circuit fault, fast-acting fuses located in each inverter branch isolate defected branch. The controller can also detect the existence of a short circuit fault by monitoring the current rate of change. Isolating the defective branch by a fuse leads to an open circuit case that is detected by controllers and reconfiguration action is taken place 
after detection to transfer the load to a redundant branch Figure. 9.3. shows the block diagram for the detection and identifying the algorithm.

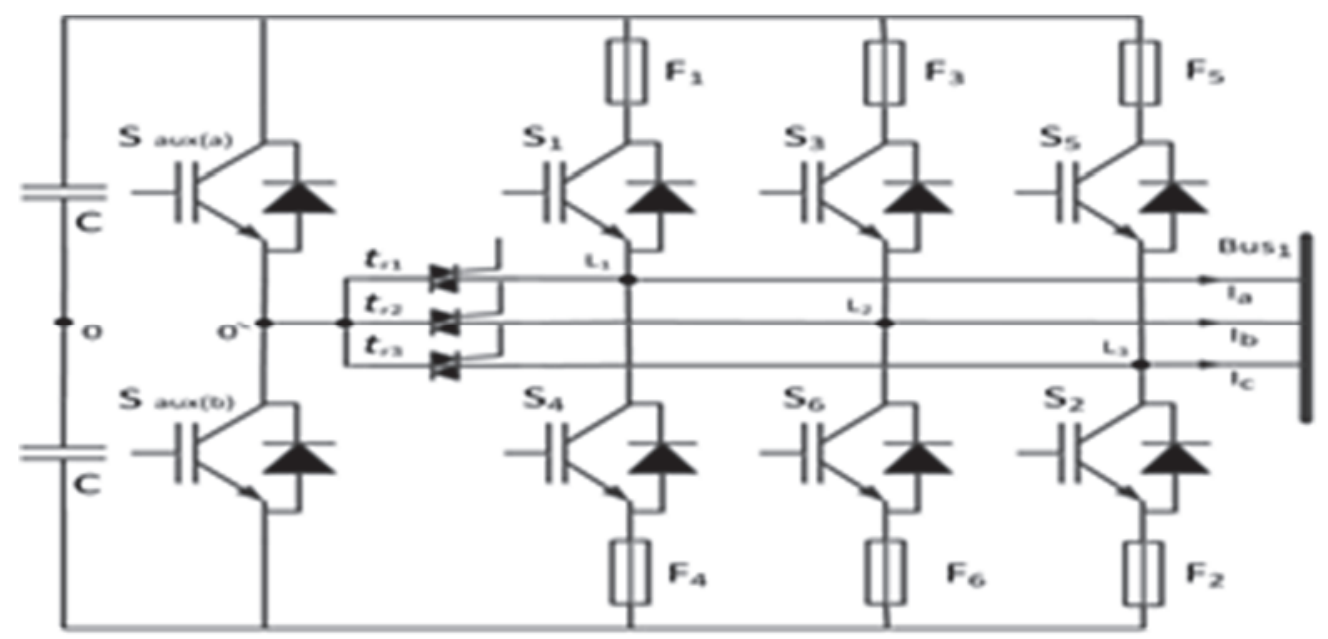

Figure 9.2 The modified inverter topology in the healthy mode

\subsection{Reconfiguration technique}

Isolation of the faulty branch and reconfiguration of the circuit after disconnection is done using the inverter topology shown in Figure 9.2. This inverter topology has four branches composed of switches $\mathrm{S}_{1} \mathrm{~S}_{4}, \mathrm{~S}_{3} \mathrm{~S}_{6}, \mathrm{~S}_{5} \mathrm{~S}_{2}$ and $\mathrm{S}$ aux_(a)

$\mathrm{S}$ aux (b). The fourth branch, composed of switches $\mathrm{S}$ aux (a) $\mathrm{S}$ aux (b), is used as a redundancy branch. In this configuration, there are disconnecting devices fast active fuses ( $F \mathrm{j}, \mathrm{j}=1,2,3,4,5,6)$, two in each inverter branch and three connecting devices trj (SCRs connected back to back) represented here by triacs, which are used to modify the inverter circuit after isolation of the faulty branch. Reconfiguration of the inverter will be done by disconnection of the faulty branch and connecting the redundancy branch instead. This can be illustrated as the following: 


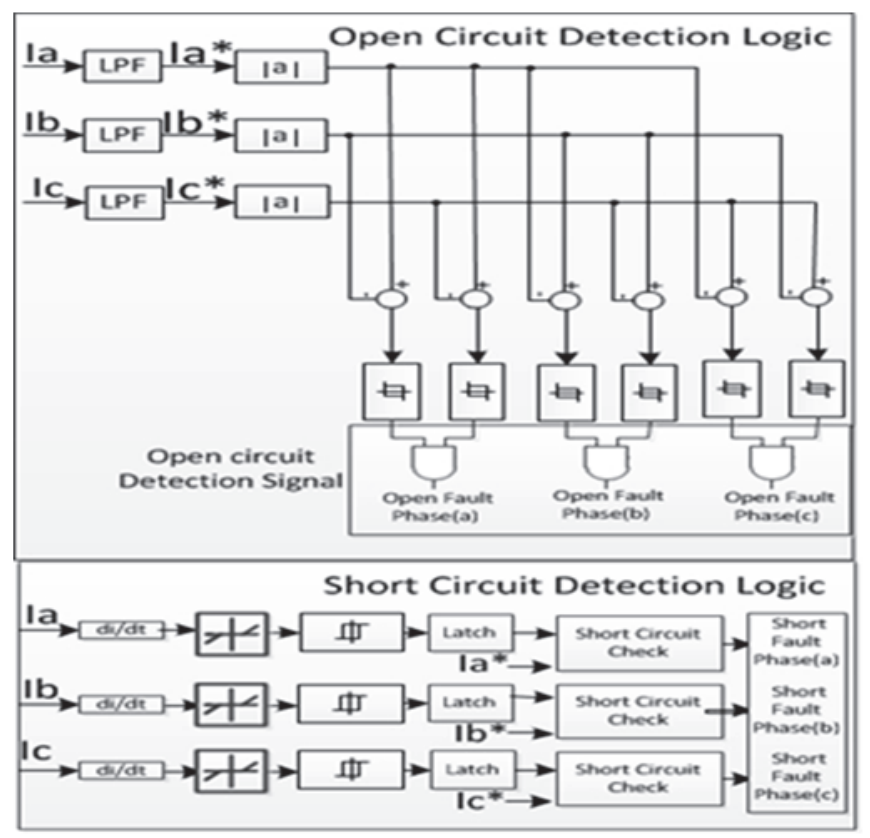

Figure 9.3 Detection and identification block diagram

\subsubsection{Disconnection of the faulty switch}

Isolating the faulty switch by disconnecting the branch which contains this switch. This process has two different ways related to the type of fault. If the fault is an open circuit fault, disconnection is performed by disabling the PWM signal for the faulty branch. Otherwise, if the fault short circuits the faulty branch, which contains the faulty switch, it will be isolated automatically because the fast-acting fuses on this branch will be blown up as a result of large short circuit current passing on it. This massive current because of a short circuit on the DC bus during a short circuit on any switch.

\subsubsection{Connection of the redundancy branch}

After the disconnection processes for the faulty branch, the reconfiguration is obtained by using the triac $\mathrm{t} r \mathrm{j}$ that is triggered on, interconnecting point $(\mathrm{Li}, \mathrm{i}=1,2,3)$ to point 0 
as given in Figure 9.4. This describes the shape of the inverter after the fault is disconnected and reconfiguration is wholly done. In this figure, a short circuit fault happened for switch $\mathrm{S}_{1}$ or switch $\mathrm{S}_{2}$ so the branch which contains these switches is disconnected and the redundancy branch is connected instead of the faulty branch by connected the Triac switch tr1. Reconfiguring the circuit after fault disconnection is necessary to continuously feed the utility grid by renewable energy instead of disconnecting a large amount of energy suddenly from the grid and causing stability problems.

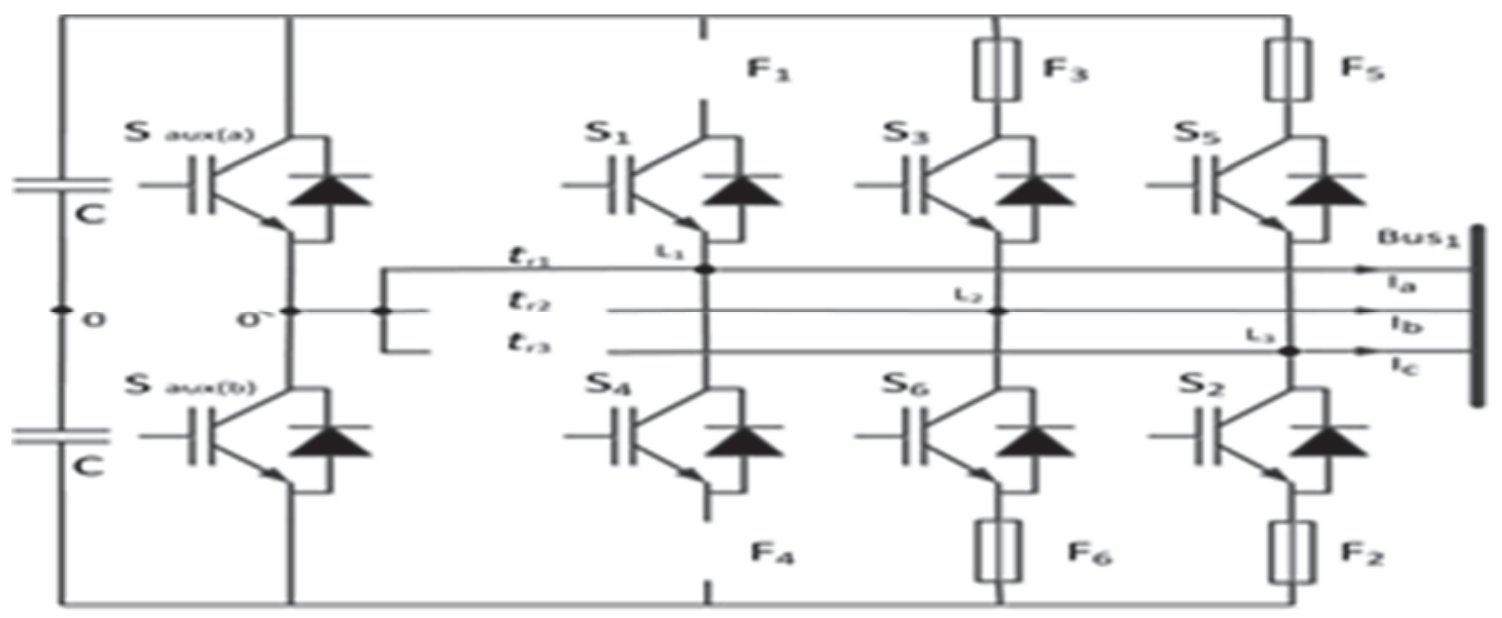

Figure 9.4 the modified inverter topology in the post fault mode

\subsection{Simulation result}

\subsubsection{Open Circuit Fault}

It is noticed that the phase voltage and current of the three-phase output of the inverter have been affected by an open circuit fault occurs in any of the inverter switches. However, in the case of an open circuit fault, the change of the output fault has not a significant effect as the output current because of the connection to the grid so there is a voltage still appears from the grid side. This appears in the following result: 
- Switch $\mathrm{S}_{1}$ Open Circuit As shown in Figure 9.5, the inverter works in regular operation until the time $(t=0.52 \mathrm{Sec})$. At $\mathrm{t}=0.52$, an open circuit fault occurs in switch $\mathrm{S} 1$ which connect phase (a) by the positive terminal of the DC BUS. The simulation shows that the current phase (a) has a negative DC component and appears disconnected on the positive half cycle. While phase (b) current has a positive DC component, phase (c) current has a negative DC component but with complete operation during the full cycle. Based on previous notices, the detection algorithm generates a detection signal at $\mathrm{t}=$ 0.59. This detection signal triggers a reconfiguration process connecting the point L1 to point $0 `$ to connect the redundant branch to phase (a).
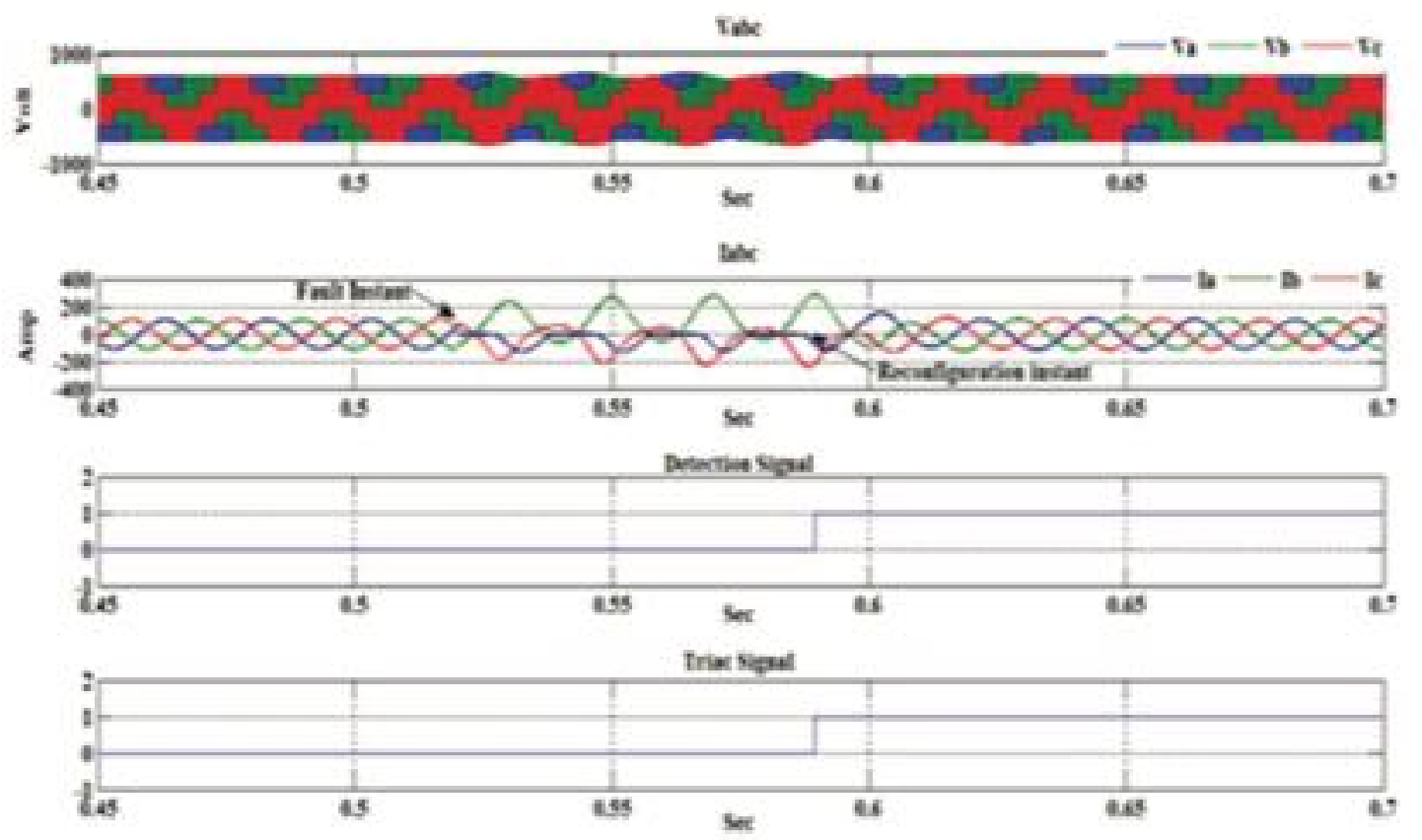

Figure 9.5 The behavior of grid-tie inverter topology with switch S1 open circuit

- Switch S4 Open Circuit

As shown in Figure 9.6, the inverter works in regular operation until time $\mathrm{t}=0.52 \mathrm{Sec}$. At $t=0.52$, an open circuit fault occurs in switch $\mathrm{S} 4$, which connect phase (a) by the 
negative terminal of the DC BUS. As described before, this occurs with switch S1 as an open circuit. The fault produces DC components, but with an opposite sign for the DC component of the three-phase current in case of S1 open circuit and with complete disconnection for the current of phase (a) on the negative half cycle. So the detection signal takes the same action by trigger reconfiguration process connecting the redundant branch to phase (a).

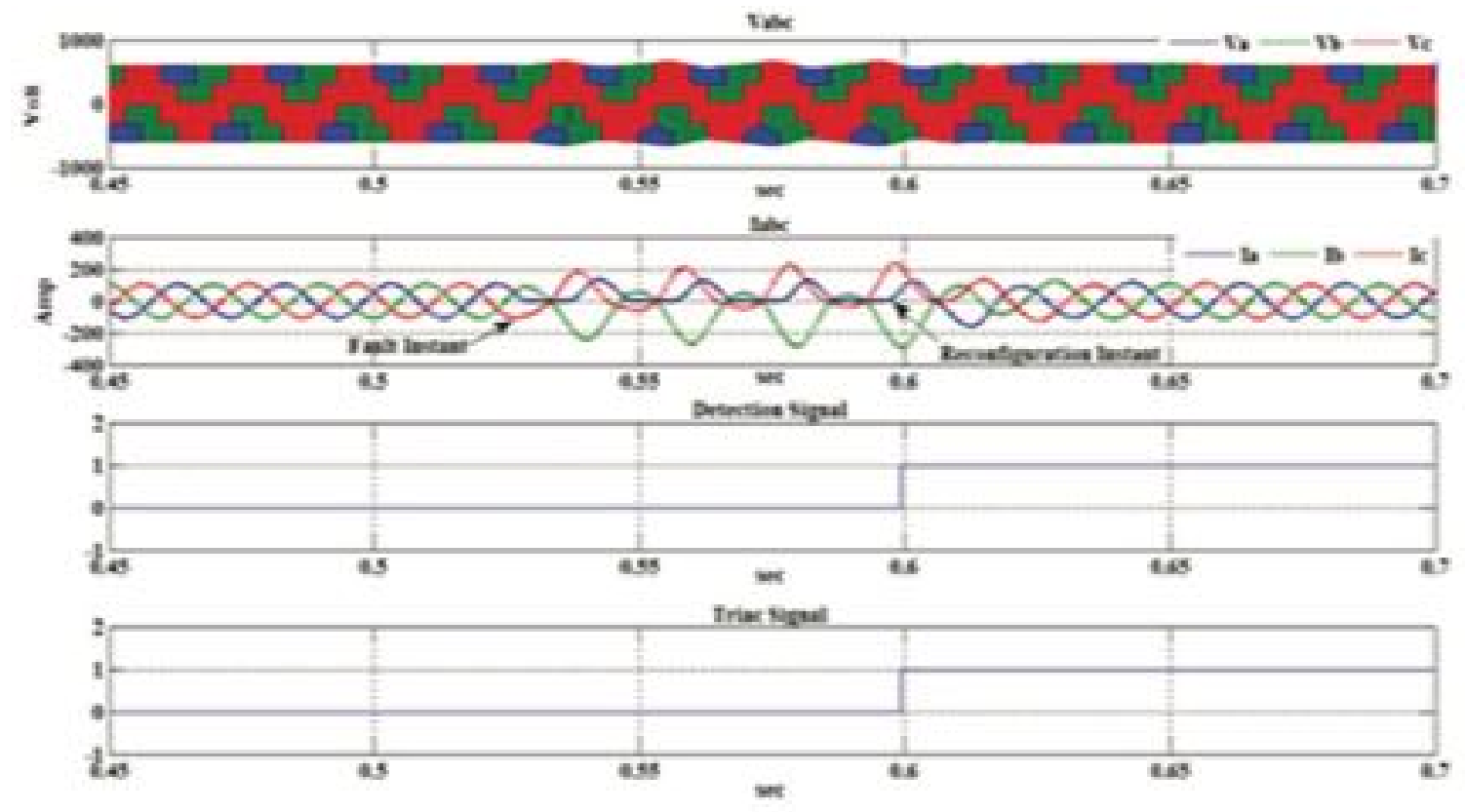

Figure 9.6 The behavior of grid-tie inverter topology with switch S4 open circuit

\section{- Switch S3 Open Circuit}

As shown in Figure 9.7, the inverters work in regular operation until the time $(\mathrm{t}=0.52$ $\mathrm{Sec})$. At $\mathrm{t}=0.52$, an open circuit fault occurs in switch S3 which connects phase (b) by the positive terminal of the DC BUS. The simulation shows that the current phase (b) has a negative DC component and appears disconnected on the positive half cycle. While phase (c) current has a positive dc component, phase (a) current has a negative dc component but 
with complete operation during a full cycle. Based on previous notices, the detection algorithm generates a detection signal at $\mathrm{t}=0.59$. This detection signal trigger reconfiguration process connects the point L2 to point 0 ' to connect the redundant branch to phase (b).
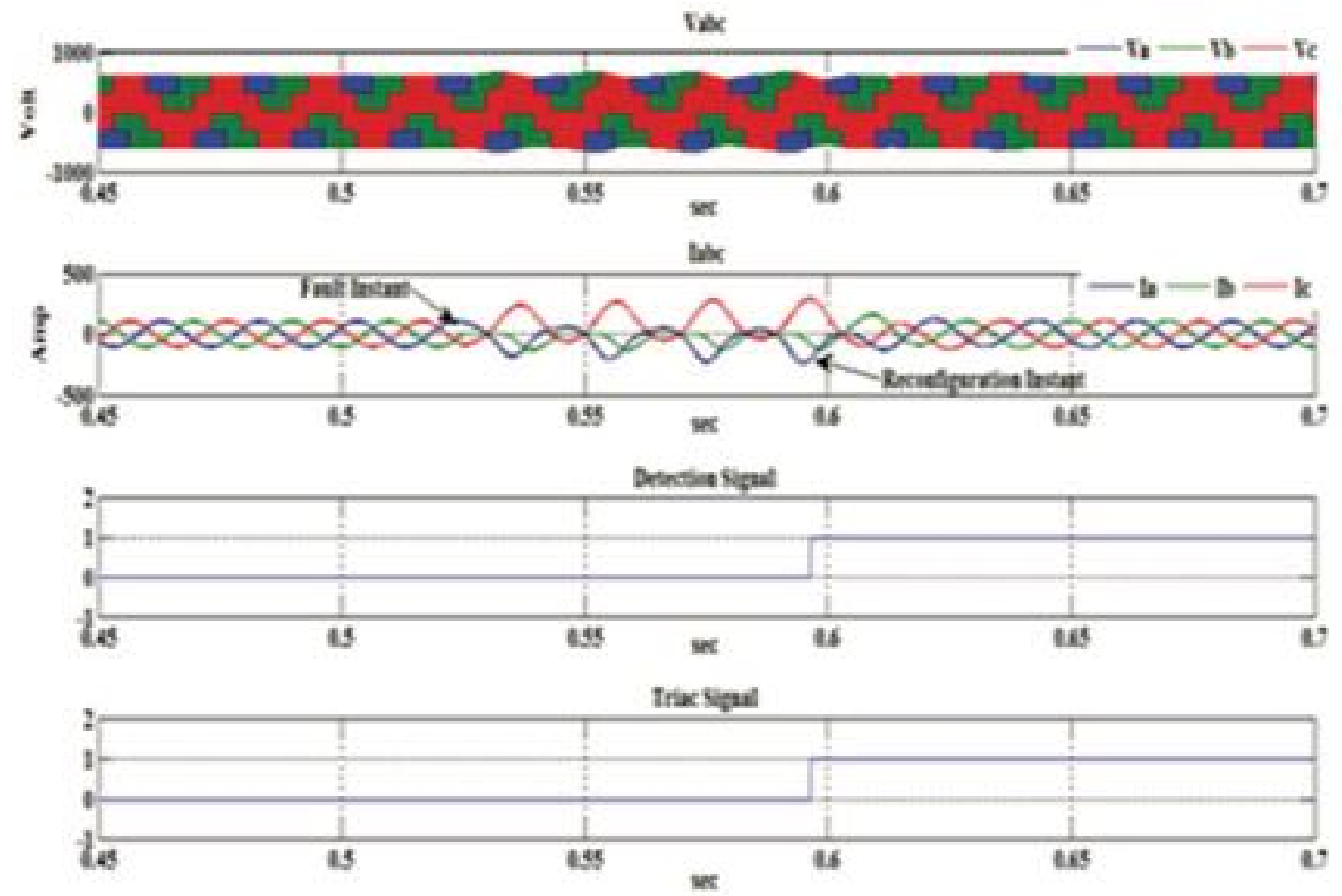

Figure 9.7 The behavior of grid-tie inverter topology with switch S3 open circuit

- Switch S6 Open Circuit

As shown in Figure 9.8, the inverter works in normal operation until time $\mathrm{t}=0.52 \mathrm{Sec}$. At $\mathrm{t}=0.52$, an open circuit fault occurs in switch S6, which connects phase (b) by the negative terminal of the DC BUS. As described before, this occurs with a switch $\mathrm{S}_{3}$ open circuit. The fault produces DC components, but with an opposite sign for the DC 
component of the three-phase current in case of $\mathrm{S}_{3}$ as an open circuit and with complete disconnection for the current of phase (b) on the positive half cycle.

So the detection signal takes the same action by triggering reconfiguration processes connecting the redundant branch to phase (b).
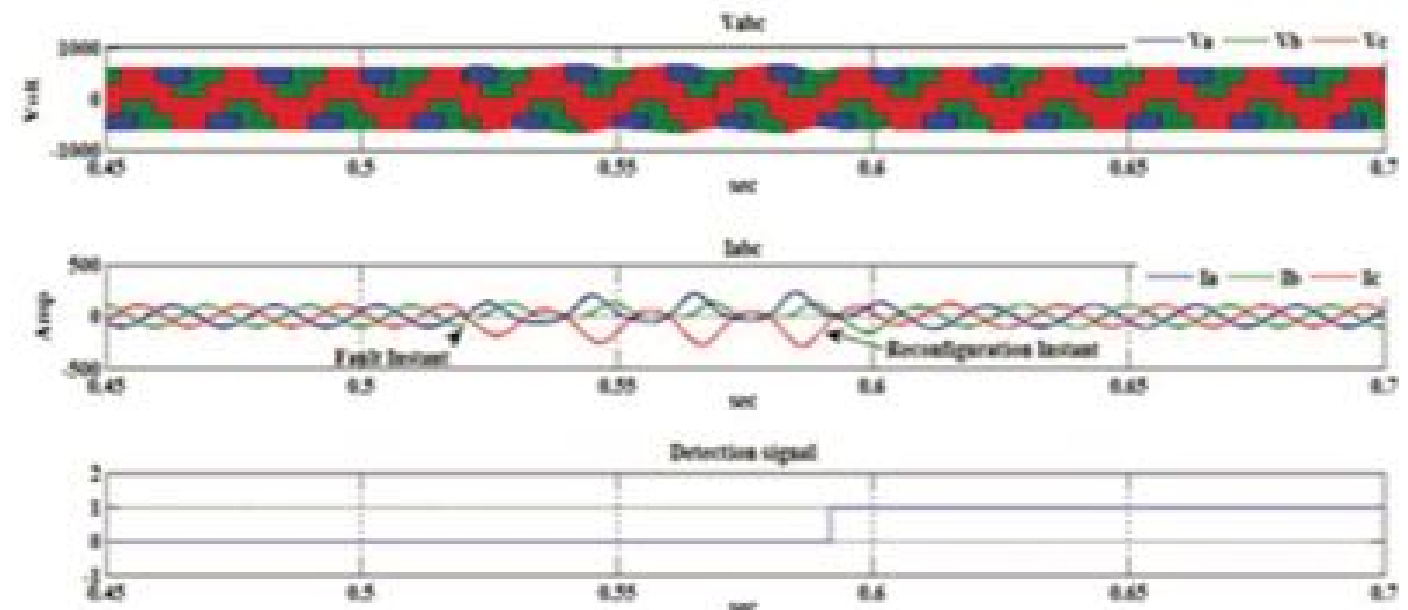

Trtat Sipal

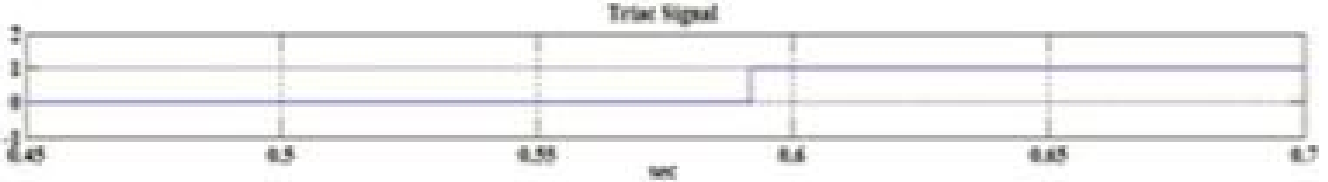

Figure 9.8 The behavior of grid-tie inverter topology with switch S6 open circuit

\subsubsection{Short Circuit Fault}

When a short circuit occurs, the distortion in both the phase voltage and current appears. The three-phase current will be rapidly increased. The detection algorithm can find the short circuit fault but cannot identify the faulty phase until the fuses on the faulty branch blow, and one of the phases currently becomes zero. The following results will appear these effects:

- Switch S1 Short Circuit 
As shown in Figure 9.9, the inverter works in regular operation until the time $(\mathrm{t}=0.52$ $\mathrm{Sec})$. At $\mathrm{t}=0.52$, a short circuit fault occurs in switch $\mathrm{S} 1$. The simulation shows that the current of phase (a) has a negative DC component, while phase (b) current and phase (c) current has positive DC components. Because of the rapid rate of change for the current, the detection algorithm can distinguish the short circuit fault as shown at $t=0.5242 \mathrm{sec}$ but without identifying the faulty branch until the fast-acting fuses on that branch burned. As shown at $\mathrm{t}=0.5342$, the current phase (a) became zero. Based on previous notices. The detection algorithm generates a Triac signal at $\mathrm{t}=0.5542 \mathrm{sec}$. This triac signal triggers a reconfiguration process connecting the point L1 to point 0 ' to connect the redundant branch to phase (a).

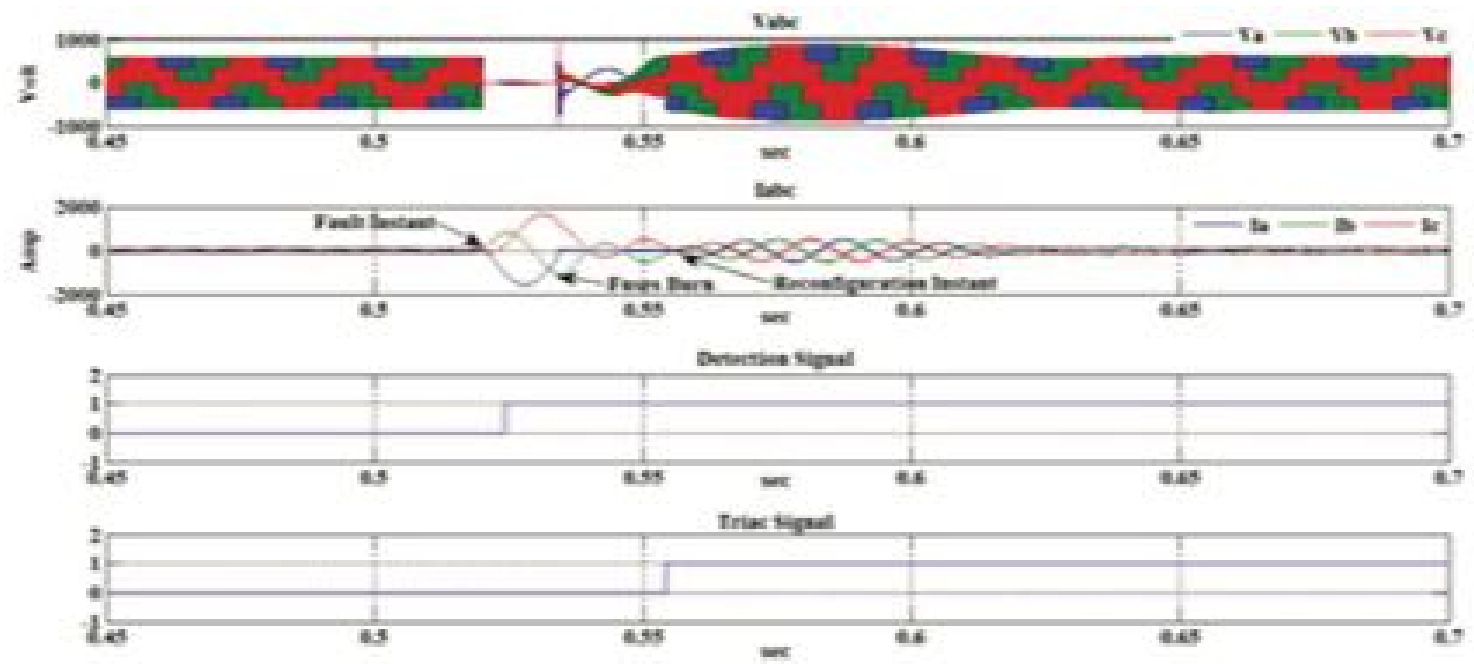

Figure 9.9 The behavior of grid-tie inverter topology with switch S1 short circuit

- Switch S4 Short Circuit

As shown in Figure 9.10, the inverter works in regular operation until the time $(\mathrm{t}=0.52$ $\mathrm{Sec})$. At $\mathrm{t}=0.52 \mathrm{Sec}$, a short circuit fault occurs in switch S4. As described before, switch $\mathrm{S} 1$ is short-circuited. The fault produces DC components as same as the DC component of 
the three-phase current in case of an $S_{1}$ short circuit based on the same sequence taken in the previous case. The detection algorithm detects the faulty case at $t=0.5242 \mathrm{sec}$ and identifies the faulty branch at $\mathrm{t}=0.5342 \mathrm{sec}$ when the current of phase (a) becomes zero and sends a Triac signal which triggers a reconfiguration process connecting the redundant branch to phase (a).

- Switch S3 Short Circuit

As shown in Figure 9.11, the inverter works in regular operation until the time $(\mathrm{t}=$ $0.52 \mathrm{Sec})$. At $\mathrm{t}=0.52$, a short circuit fault occurs in switch $\mathrm{S} 3$. The simulation shows that the current of phase (b) has a positive DC component, while phase (a) current has a negative DC component and phase (c) current has a positive dc component. Because of the rapid rate of change for the current, the detection algorithm can distinguish the short circuit fault as shown at $\mathrm{t}=0.5242 \mathrm{Sec}$ and identifies the faulty branch as shown at $\mathrm{t}=0.5342$ when the current of phase (b) became zero. Based on previous notices, the detection algorithm generates a Triac signal at $\mathrm{t}=0.5542 \mathrm{sec}$. This triac signal triggers a reconfiguration process connecting the point $\mathrm{L} 2$ to point 0 ' to connect the redundant branch to phase (b). 

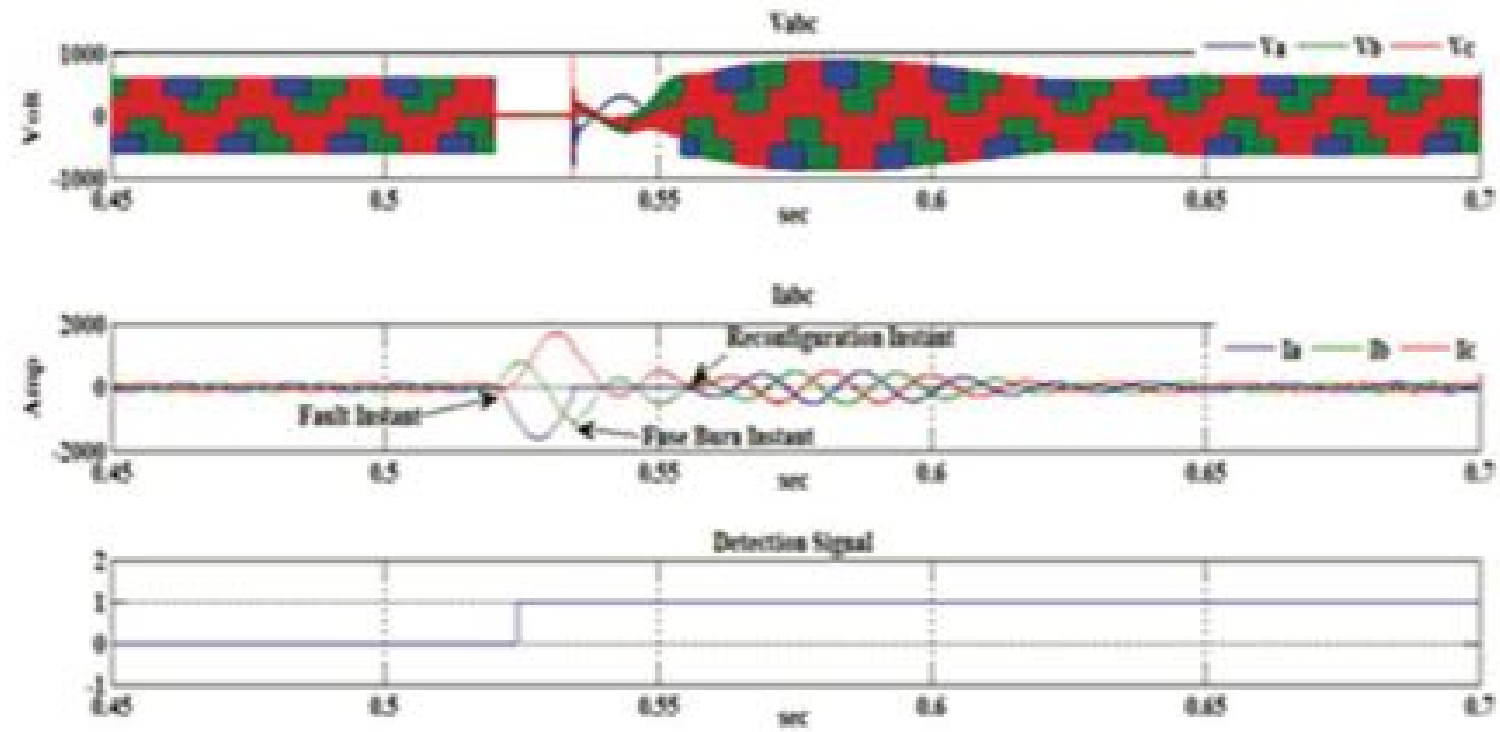

trias Sipal

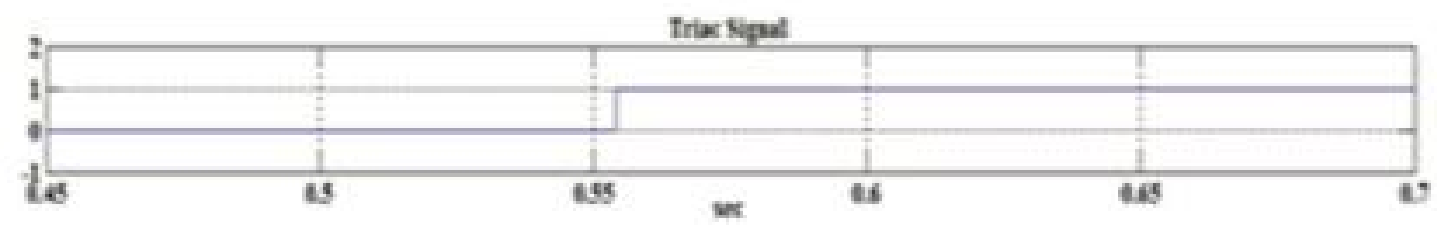

Figure 9.10 The behavior of grid-tie inverter topology with switch S4 short circuit
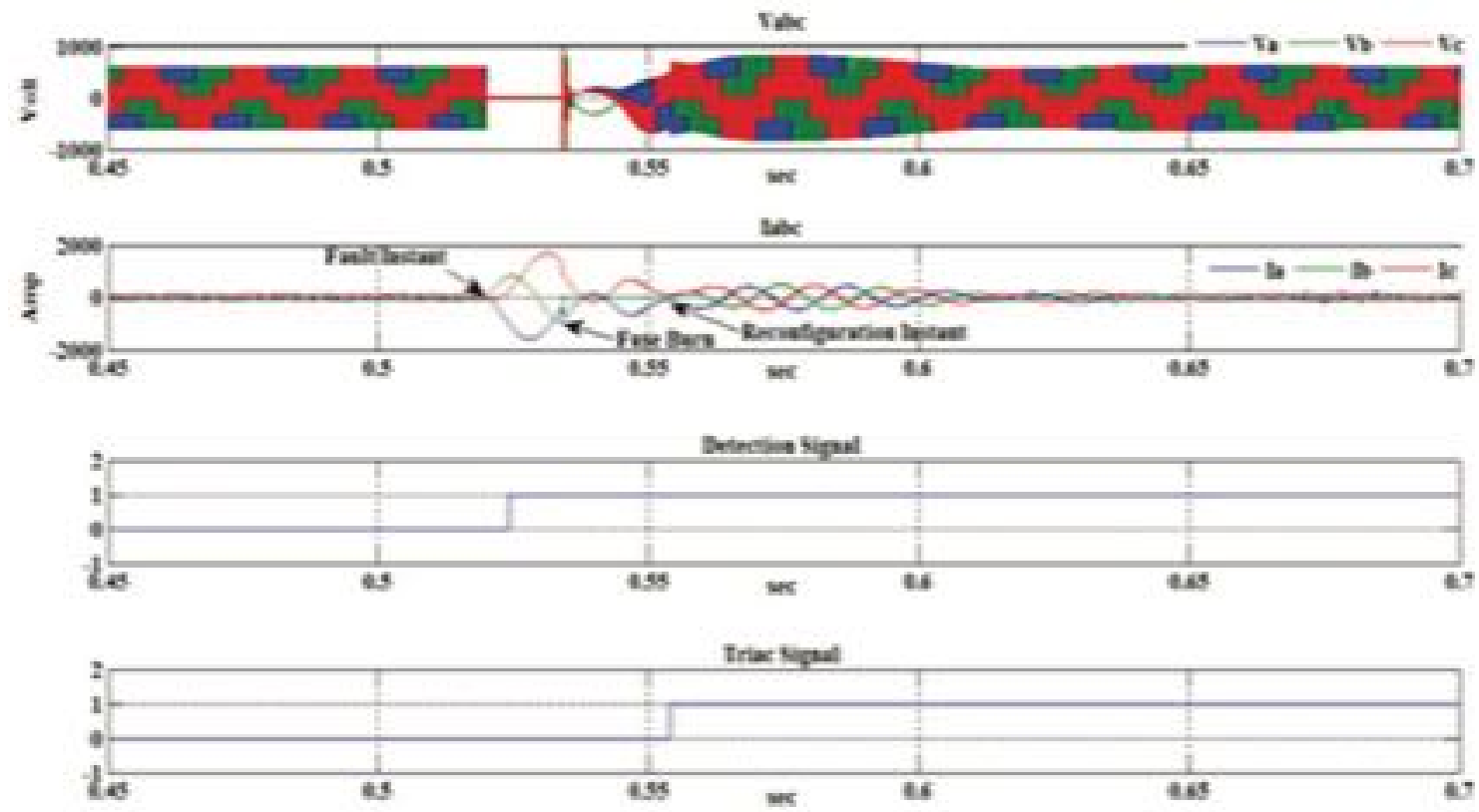

Figure 9.11 The behavior of grid-tie inverter topology with switch S3 short circuit 
- Switch S6 Short Circuit

As shown in Figure 9.12, the inverter works in regular operation until the time $(\mathrm{t}=0.52$ $\mathrm{Sec})$. At $t=0.52 \mathrm{Sec}$, a short circuit fault occurs in switch S6. As described before, switch S3 is short-circuited. The fault produces DC components as same as the DC component from the DC component of the three-phase current in case of the S1 short circuit. Based on the same sequence taken in the previous case, the detection algorithm detects the faulty case at $\mathrm{t}=0.5242 \mathrm{sec}$, identifies the faulty branch at $\mathrm{t}=0.5342 \mathrm{sec}$ when the current of phase (a) became zero and sends a Triac signal which trigger reconfiguration process connecting the redundant branch to phase (a).

In the entire previous fault cases, after the reconfiguration of the inverter return to normal operation mode, the injecting power became stable.
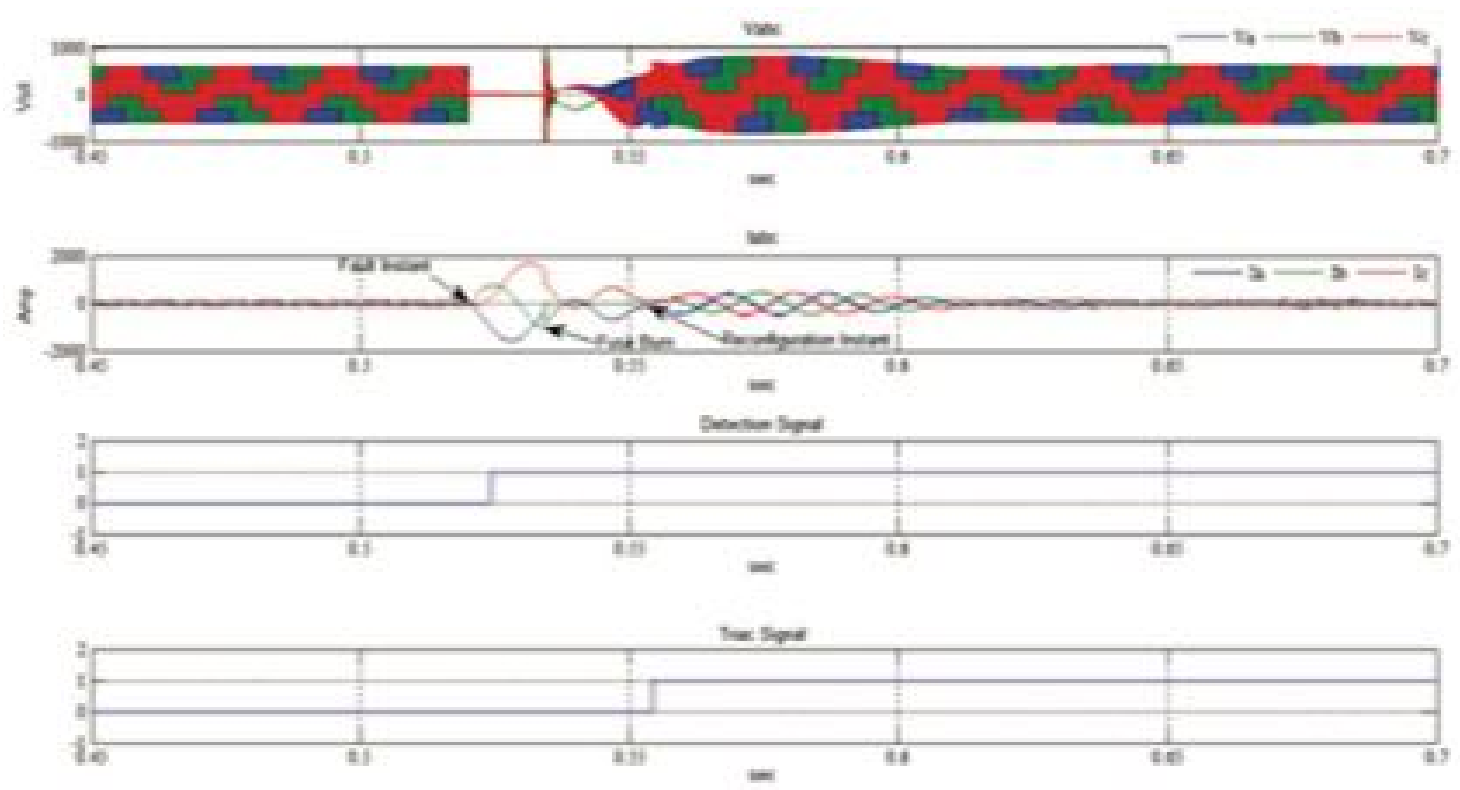

Figure 9.12 The behavior of grid-tie inverter topology with switch S6 short circuit 


\subsection{Conclusion}

This work has demonstrated the validity of the proposed fault detection strategy used to detect open or short circuit faults occurring on inverter switches and validates applying a modified inverter circuit such as the proposed inverter topology. This provides continuous feeding energy to the utility grid after a fault occur on one of the inverter switches even with a complete loss of one of the converter branches. The fault compensation is achieved by reconfiguring the power converter topology by using Triac switches, which are generally open. While one of them is closed to redefine the converter topology. In the proposed type of grid-connected inverter topology, a redundancy branch was added as hardware redundancy and the Triac switches are used to replace the faulty branch.

The proposed inverter topology may add quite a bit of complexity to the original gridtie inverter system. Nevertheless, from another side higher rates of the inverter failure result in repeated shutdown events. These unwanted shutdowns in a bulk generation unit may result in the reproduction of fault, thereby affecting the overall grid stability that means reduced the overall reliability and efficiency of the power grid. So using this fault-tolerant circuit topology for the grid-tie inverter and the proposed detection algorithm will result in avoiding the repeated shutdown periods for the system, which increases the efficiency and the reliability of the system.

The approach of this work shows an increase in the reliability of the conventional power inverter used in the grid-connected PV system by adding a redundancy branch. The work also presents a detection control algorithm that has an essential effect in avoiding a 
power stability problem in the case of a fault occurring on one of the inverter switches leading to disconnection of the PV microgrid. 


\section{Chapter 10 EXPERIMENTAL SETUP OF THE MICRO/NANO GRID TO INVESTIGATE THE DEMAND SIDE MANAGEMENT SCHEMES}

\subsection{Introduction}

This chapter presents the construction of the developed hybrid AC/DC Smart Building (Micro/Nano grid) infrastructure. This Micro/Nano grid is implemented to investigate the developed techniques for the demand-side response of a smart building to the utility needs. The Experimental setup constructed at the Energy Systems Research Laboratory, Florida International University. The main construction of the Micro/Nano grid includes PV Emulator, DC/DC boost converter for PV_MPPT, Programmable DC Power Supply, DC/AC grid-tie converter, synchronous generator, Load Emulator (fixedstep control loads, Variable step control loads), energy storage devices such as Flywheel and batteries.

\subsection{Source Components of the Micro/Nano grid}

\subsubsection{PV Emulator}

Installation of a PV system needs an accurate design and evaluation test for the performance of its modules before using it in a system. This could be fulfilled with high accuracy in the laboratory without using commercial PV modules by using a PV emulator. The PV emulator introduces a suitable tool which easily permits emulating PV system component with different parameters, teaching its characteristic and training researchers in this field.

In this work, a $6 \mathrm{~kW}$ programmable PV emulator is designed that has the capability of emulating commercial PV modules with different parameters and under different 
environmental conditions (irradiance or temperature change), constructing a PV array based on the series and parallel numbers of modules. The PV emulator is implemented using a real-time algorithm representing the $\mathrm{PV}$ array mathematical model and generating the reference output power from a programmable DC power supply. The PV model is simulated in MATLAB and interfaced with the real world through dSPACE using an evolution graphic user interface (GUI). This laboratory PV emulator is tested with realtime execution of the PV model for the steady-state and dynamic operating conditions. The block diagram representation of the proposed PV emulator is shown in Figure.2.1. It mainly consists of a programmable DC power supply (Programmable Magna Power Supply XR series), Matlab/Simulink package, dSPACE1104 with control desk software installed on a personal computer $(\mathrm{PC})$ and resistive load with different values.

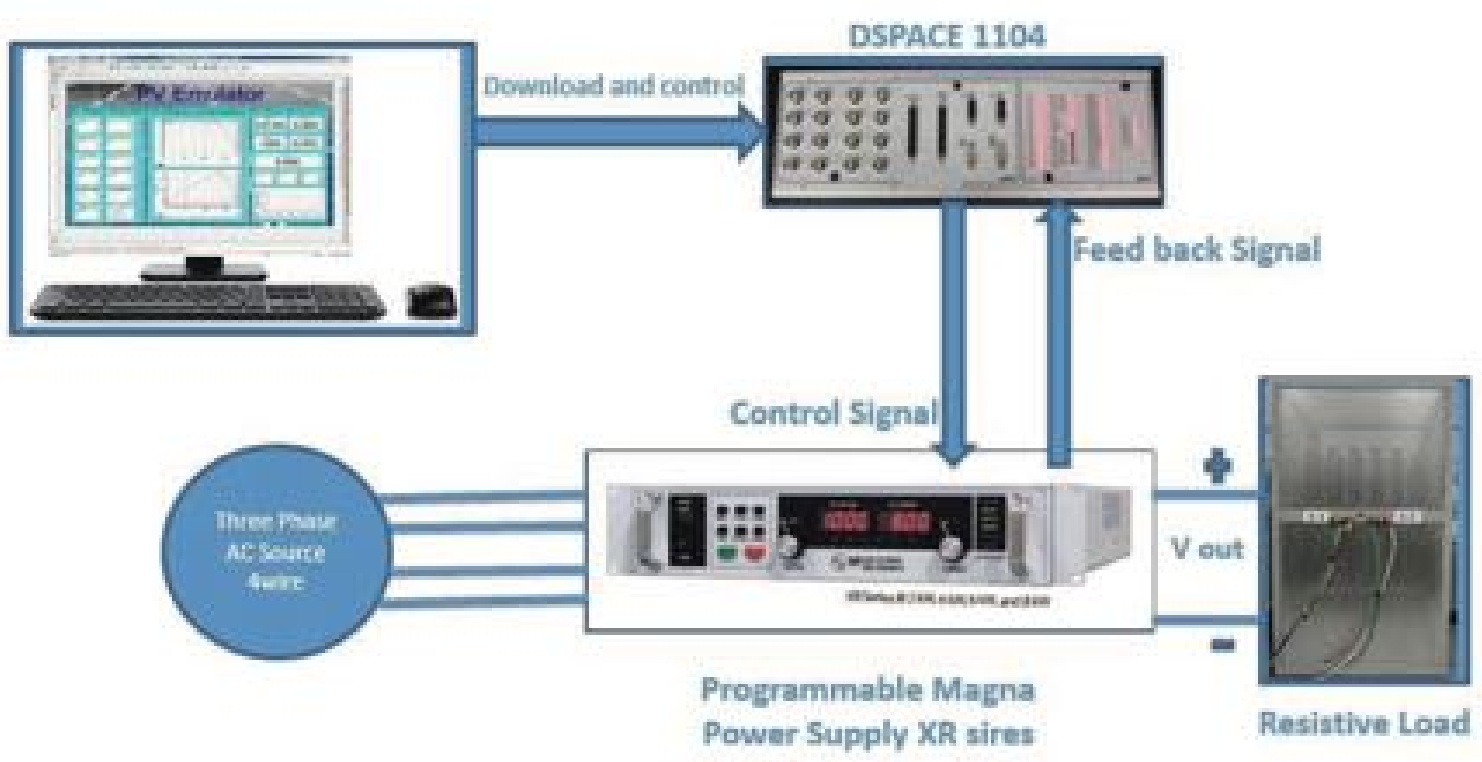

Figure 10.1 Block diagram for the proposed PV Emulator. 
The emulator GUI, shown in Figure.10.2, is developed in the dSPACE ControlDesk and allows real-time control of the environmental conditions, i.e., solar irradiance, temperature and the PV module parameter which allows emulating diverse of PV modules. It allows constructing a PV array within the available power of the Programmable DC power supply.it gives the user an intuitive feel as to how a real solar panel would react to changes in temperature, irradiance and loads.

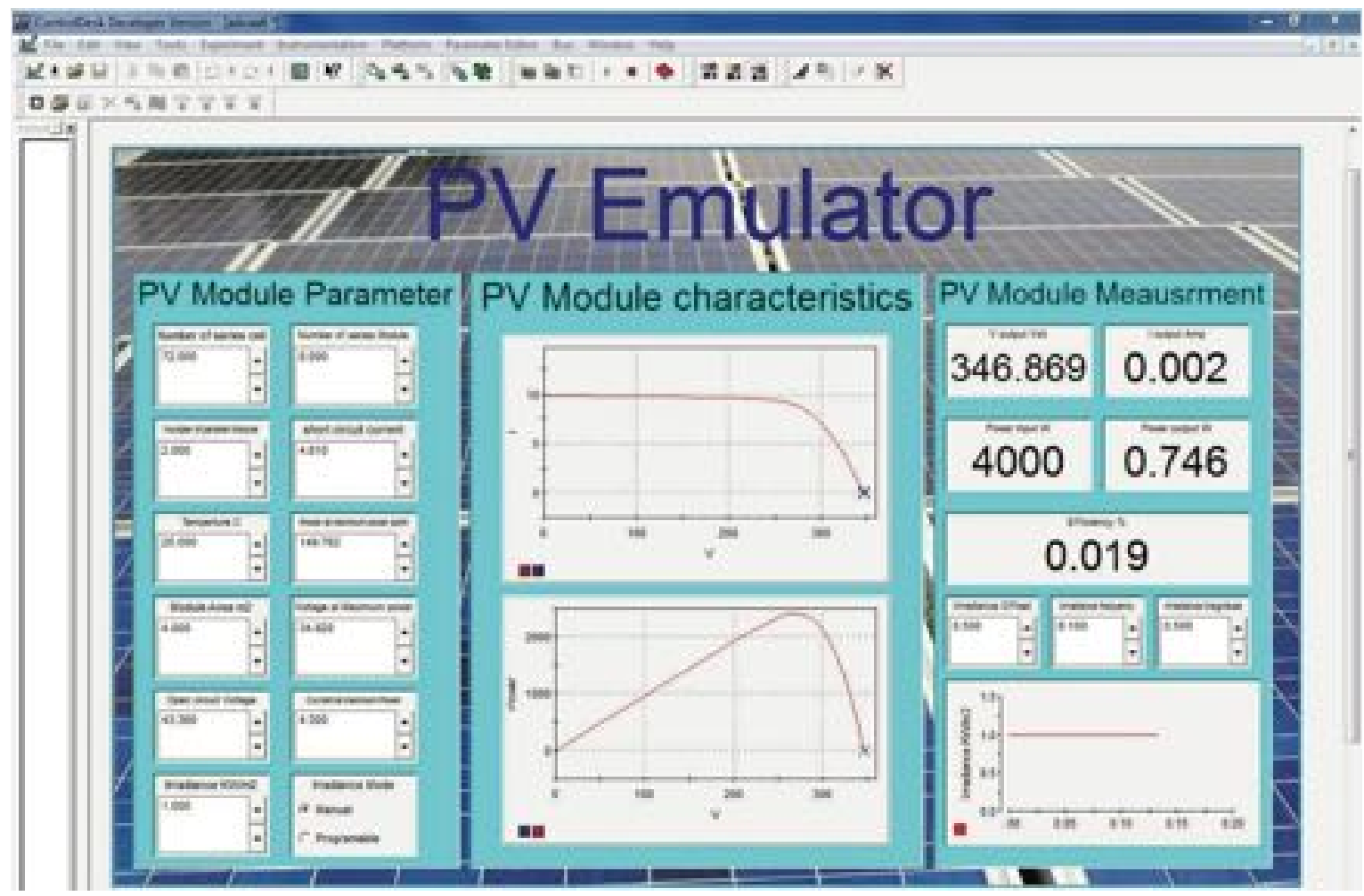

Figure 10.2 GUI of the PV emulator Using Matlab-Control Desk.

It consists of three major sections, the PV module parameter, PV module characteristics, and PV module measurement. First, module parameter section in which several numerical control buttons allow the user to change the temperature, irradiance, and module parameters such as a number of series cells per module, open-circuit voltage, short circuit current, current at maximum power, the voltage at maximum power and maximum power. 


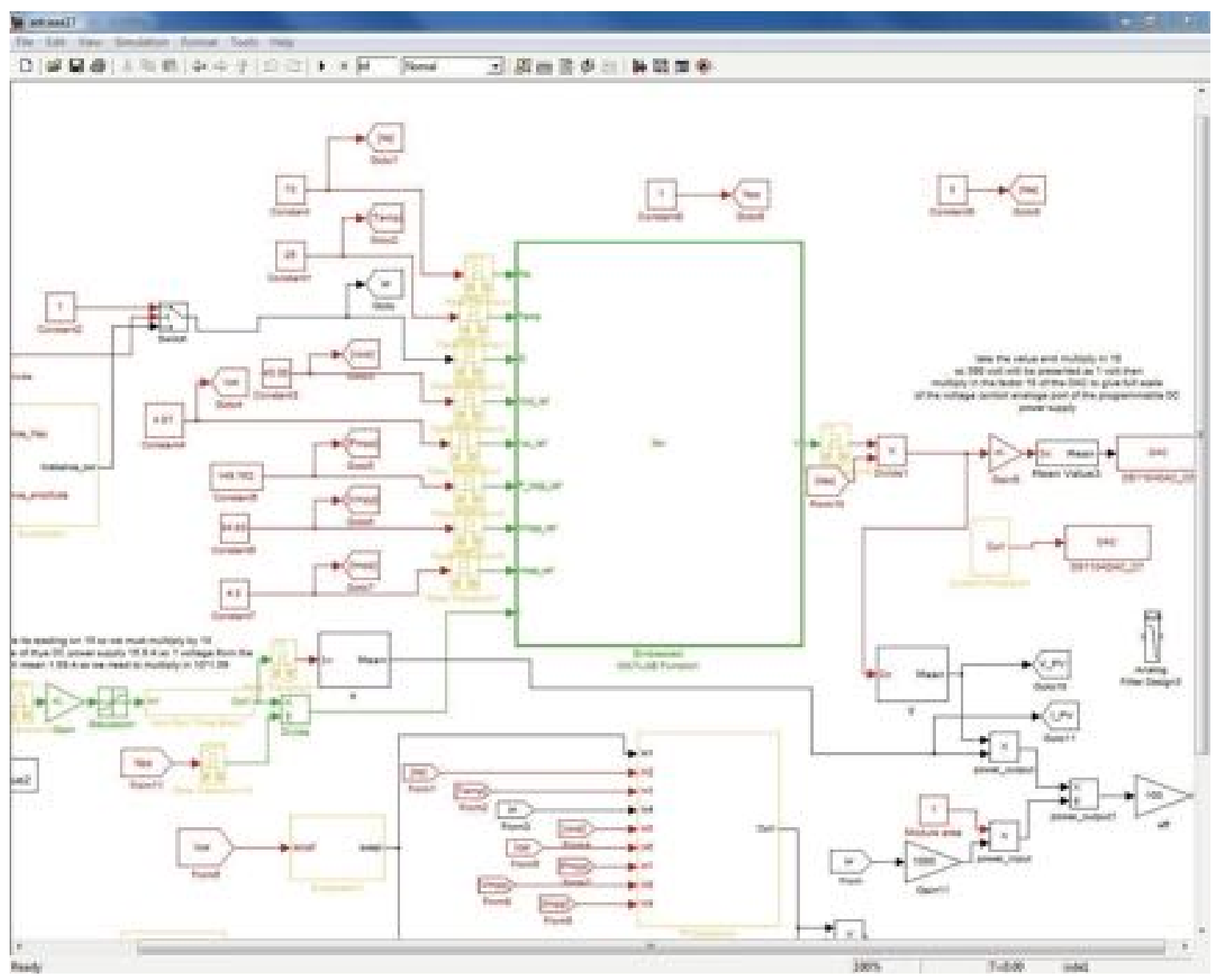

Figure 10.3 PV emulator's Matlab-Simulink model.

It gives two modes of control for the irradiance through the irradiance mode button. The user can select between two mode manual mode and programmable mode. In the case of manual mode, the user can change the irradiance manually through the irradiance button. In the case of programmable mode, the irradiance will be changed automatically in sinusoidal variation. The variation limits can be controlled by the controlled button, irradiance offset and irradiance magnitude. It is allowable to control the rate of change through the irradiance frequency button. Second, the PV module characteristics section in which the theoretical curves of the current-voltage and power-voltage for the PV array are displayed depending on the input parameters. The operating point is displayed through the 
blue cross on the curves, depending on the load power. Last, the PV module measurement section in which the reading of the total voltage, current, power and efficiency for the PV array are displayed.

Figure 10.3. represent the Simulink model of the PV, in which the PV model represented with the Matlab function block. All the parameters changing the characteristic of the PV output are input reference to the PV model to allow changing all of them to represent different PV modules.

In order to run the system, the following steps should perform:

1- Open the Matlab model shown in figure 10.3. which exists at $\mathrm{C}: \backslash$ desktop $\backslash$ PV_simulink.

2- Build the model from the MatLab taskbar.

3- Open the control desk program.

4- Open the GUI, as shown in figure 10.2.

5- Put the GUI in the running mode.

6- Run the programmable DC power supply shown in figure 10.4 .

7- Change the parameter of the model from the GUI and notice the change at the PV characteristics.

\subsubsection{Programmable DC Power Supply}

The DC power supply used shown in Figure.10.4. It is XR Series Magna Power Supply, and the input voltage is three phases 208 volt $\mathrm{AC}$, the rated output power $6 \mathrm{KW}$, the rated output current 15.9 Amp, and the rated output voltage 375 Volt. 


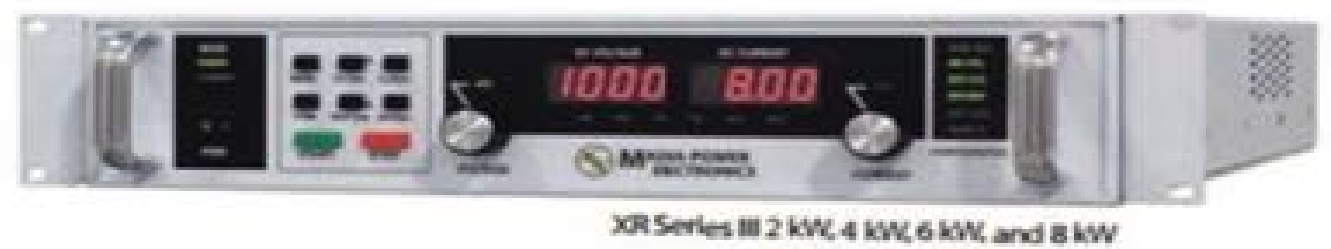

Figure 10.4 Programmable DC Power Supply.

\subsubsection{Synchronous Generator}

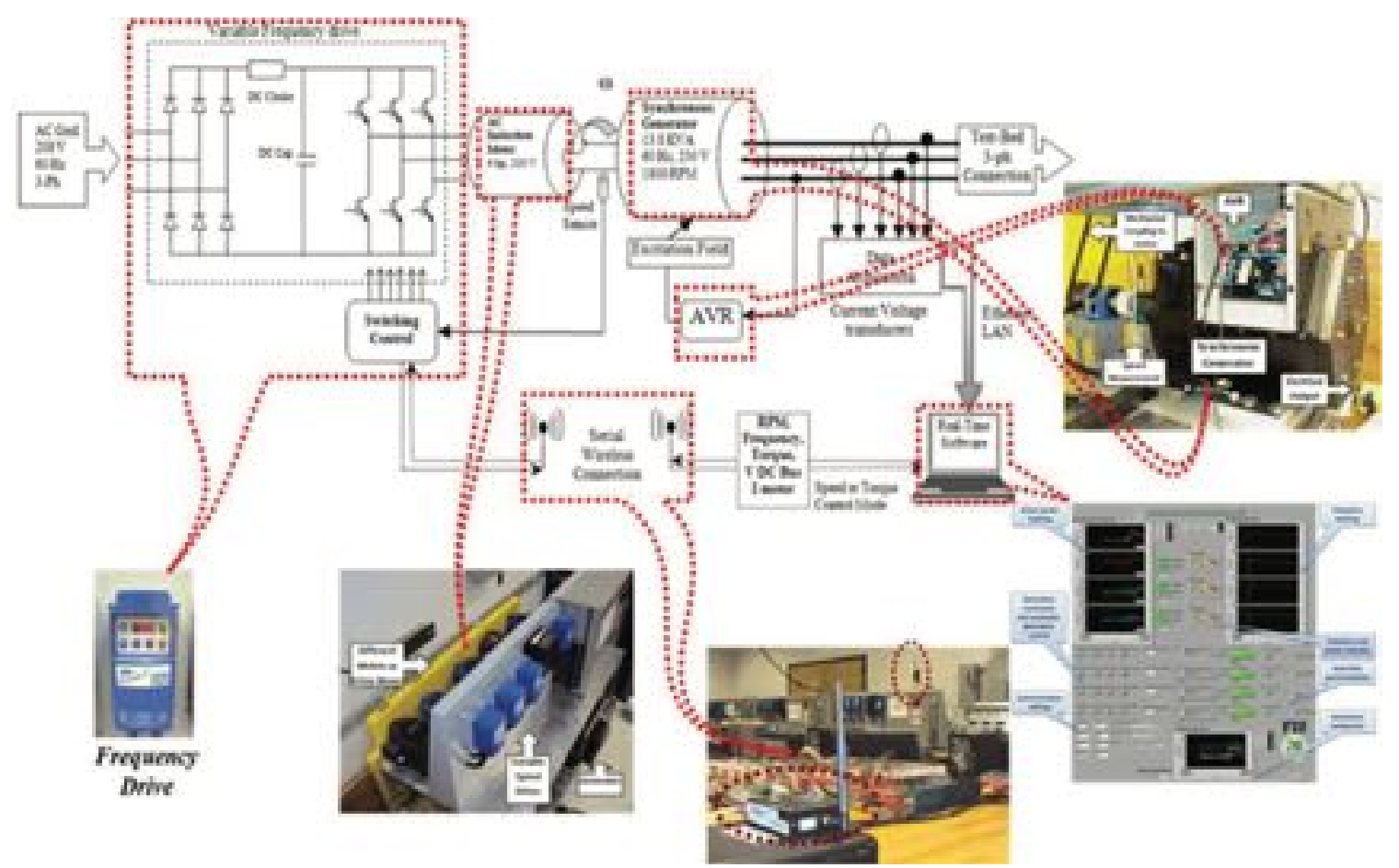

Figure 10.5 Synchronous generator at the ESRL Smart Grid Testbed.

A 13.8-kVA, 60-Hz, 208-V, and $1800 \mathrm{Rpm}$ AC are representing the specification of the synchronous generator generation unit. The generator is equipped with a half-wave phasecontrolled thyristor-type automatic voltage regulator. The generating station has five different types of motor drives acting as five different prime movers to enable the implementation of various generation control strategies. The frequency drive part follows "vector speed control" that serves to maintain the frequency stable. In order to keep the power-voltage buses, the prime mover of the generator is operated by a torque-controlled 
frequency drive, which adjusts the active power of the generation station to keep the voltage signal within the accepted value. A PLC controls the speed reference and the torque of the frequency machine. The specifications of the synchronous generator are shown in Table 10.1. Figure 10.5. Indicates the synchronous generator set for the frequency machine and the voltage regulator.

Table 10.1 Synchronous generator parameters.

\begin{tabular}{|c|c|c|}
\hline Component & Parameter & Specification \\
\hline \multirow{3}{*}{$\begin{array}{c}\text { Synchronous } \\
\text { Generator }\end{array}$} & $\mathrm{S}_{\mathrm{N}}$ & $3 \mathrm{kVA}$ \\
\cline { 2 - 3 } & $\mathrm{VN}_{\mathrm{N}}$ & $208 \mathrm{~V}$ \\
\cline { 2 - 3 } & $\mathrm{FN}$ & $60 \mathrm{~Hz}$ \\
\cline { 2 - 3 } & $\mathrm{X}, \mathrm{Rs}$ & $6.87 \mathrm{mH}, 1.45 \Omega$ \\
\hline
\end{tabular}

\subsubsection{Energy Storage}

A wide variety of energy storage technologies and ratings are used in the testbed.

\subsubsection{Lead-Acid battery}

The lead-acid battery bank storage, shown in Figure.10.6. Has a 10-110 Ah capacity (approximately $13.2 \mathrm{KW}$ ) and is equipped with a unique BMS which individually manages, balances, and conducts diagnostics on each battery module using hall-effect sensors of voltage and current. The BMS not only monitors the batteries but also extracts the defective battery and compensate its loss through the distribution of the load on other resources using power electronic converters (e.g., buck/boost converter). This system is expandable to any chemistry and quantity of batteries based on its modular characteristics. This system is 
capable of data logging and advanced protection to limit the current and voltage of each battery. The system also controls the charging profile with independent frequency, duty cycle, and magnitude.

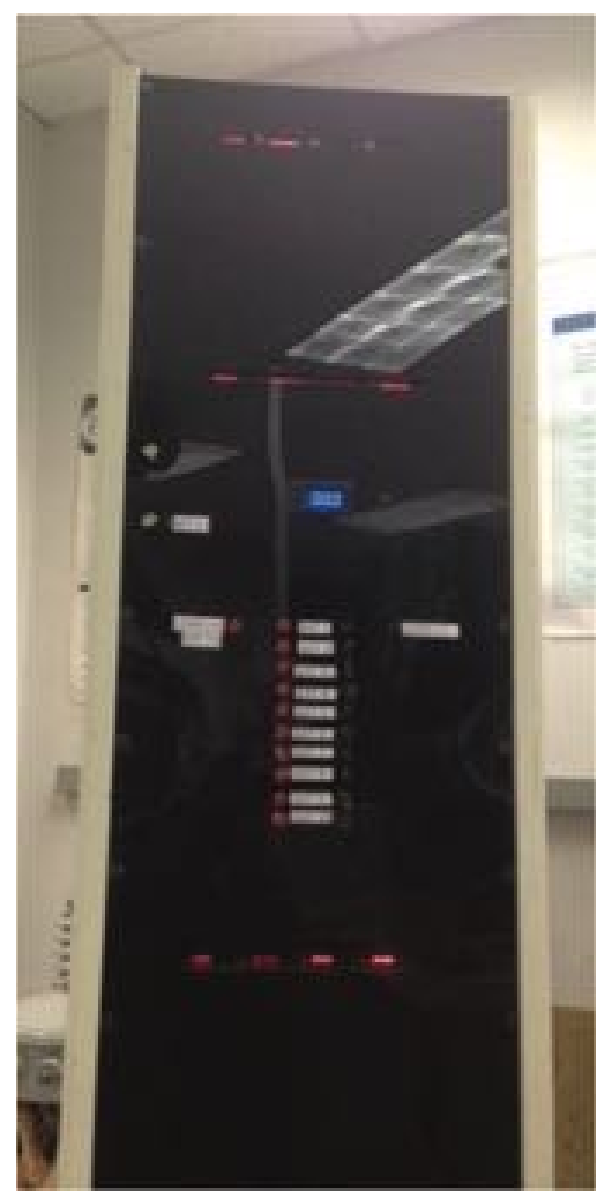

Figure 10.6 Battery Bank Stored Safely in a Closed Cabinet.

\subsubsection{Lithium-Ion battery}

Five lithium-ion battery banks are used to emulate the behavior of five individual PEVs in the smart building's car park. The lithium-ion battery bank module is shown in Figure.10.7. Each single battery bank consists of 14 lithium-ion battery cells connected in serial, with $51.8 \mathrm{~V}$ terminal voltage and $21 \mathrm{Ah}$ capacity. 


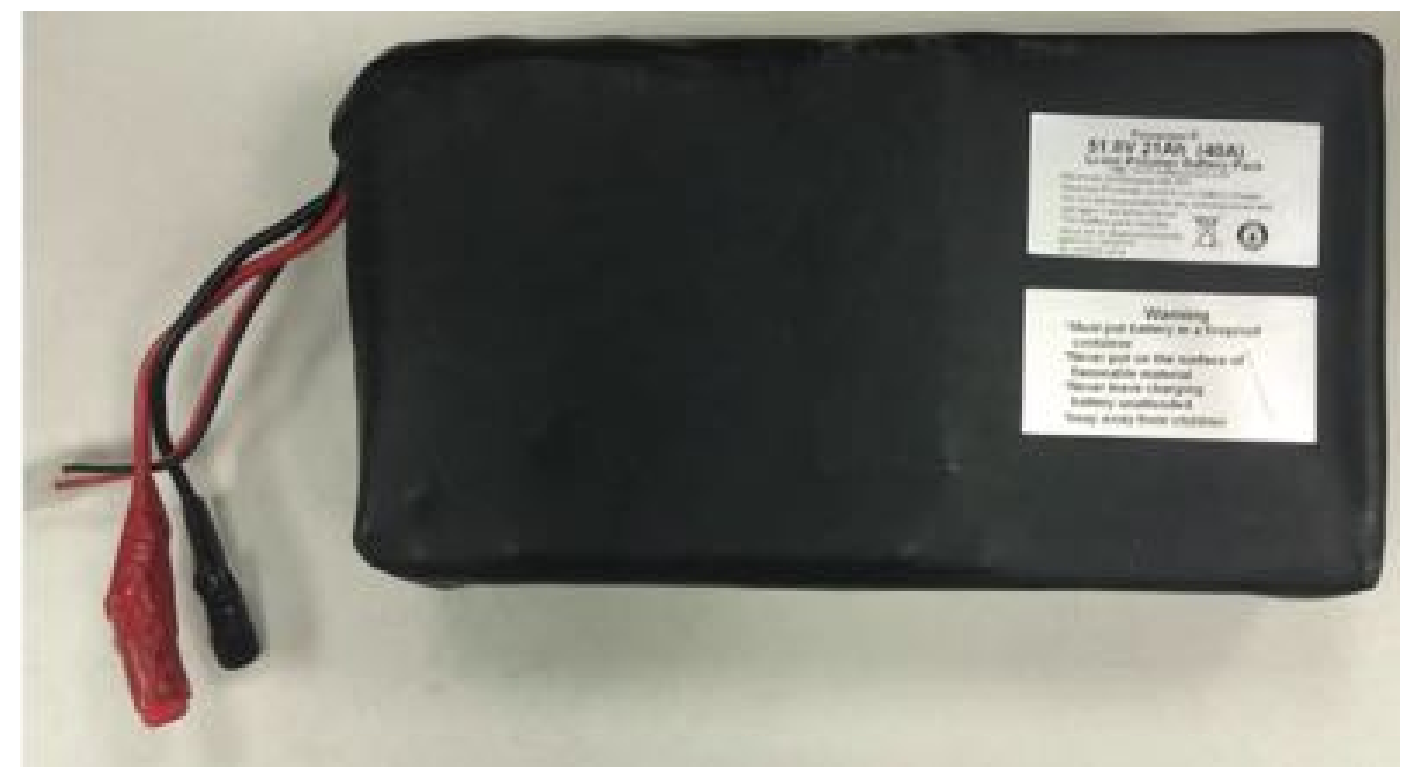

Figure 10.7 Lithium-ion battery bank module.

\subsubsection{Flywheel}

A large rotating mass coupled to a DC machine constitutes a Flywheel Energy Storage System, which works under three operating modes: charging mode, stand-by mode, and discharging mode. This setup is used to perform several studies. To name some: the power failures and outages, mitigation of pulse loads, power quality improvements, and design and performance of flywheel systems.

\subsection{Load Components of the Micro/Nano grid}

\subsubsection{Fixed step Controllable Loads}

The load module is consists of ten steps changes between 0-3 KW in the step of 300 $\mathrm{W}$ at normal voltage. These resistances can be controlled by PLC, which represented [2 $\mathrm{x}$ $72 \Omega+4 \times 144 \Omega]$. In order to maintain the frequency within the accepted range, the inductance loads are implemented based on constant inductance to avoid the derivation in 
the frequency signal. Solid-State relays are used with the ratings of $530 \mathrm{~V}$ and 25 Amps. PLC with 3-32 DC voltage can control the operation of On/Off status.

\subsubsection{Variable step Controllable Loads}

The configuration of the proposed load emulator is simple and consists of a combination of resistances with different values in a particular arrangement. The adopted topology is depicted in Figure.10.8. It can be seen that eight resistances are used with different values ( $60 \Omega, 50 \Omega, 40 \Omega, 20 \Omega, 20 \Omega$ and $1 \Omega$ ).

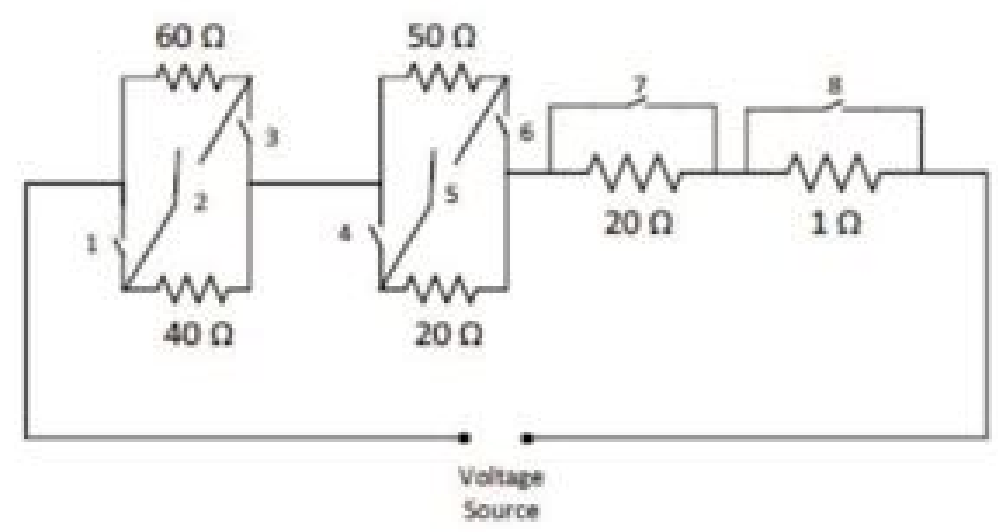

Figure 10.8 Configuration of the variable step controllable loads

The control system is implemented in a LabVIEW environment. Also, a user-friendly GUI is developed to ease the control of the system. The control signals are sent in an $8 \times 1$ vector with binary elements. Three nested loops with case structures are adopted to allow the user to select which load pattern to emulate.

\subsection{Interface Components of the Micro/Nano grid}

\subsubsection{Transmission line Model}

The transmission line models are composed of series inductors and parallel capacitors and arranged in medium length $\pi$-type. Figure 10.9. Shows the transmission line models. 

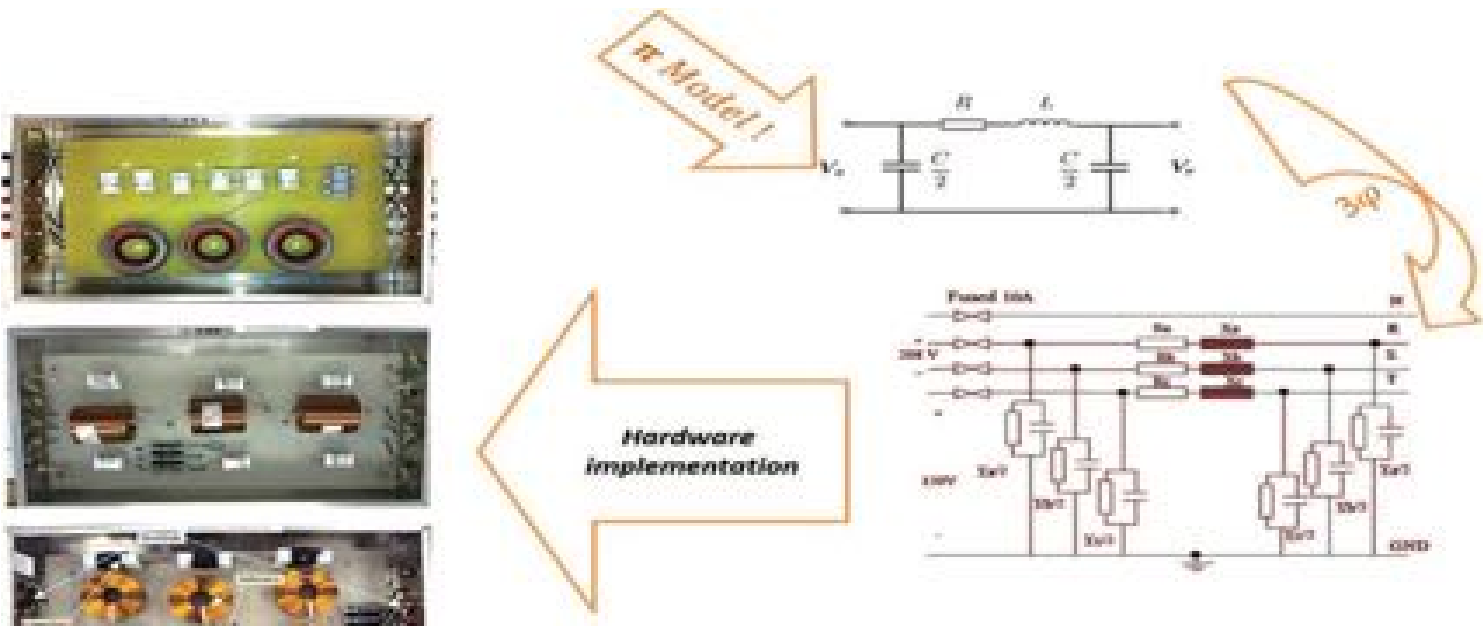

Figure 10.9 Transmission Line models.

\subsubsection{Bus Models}

The test-bed setup has 14 bus model emulator to control the switching action and emulate real power system dynamics. These modules can switch up to 15 amps per phase.

The hardware implementation of the bus model is shown in Figure 10.10.

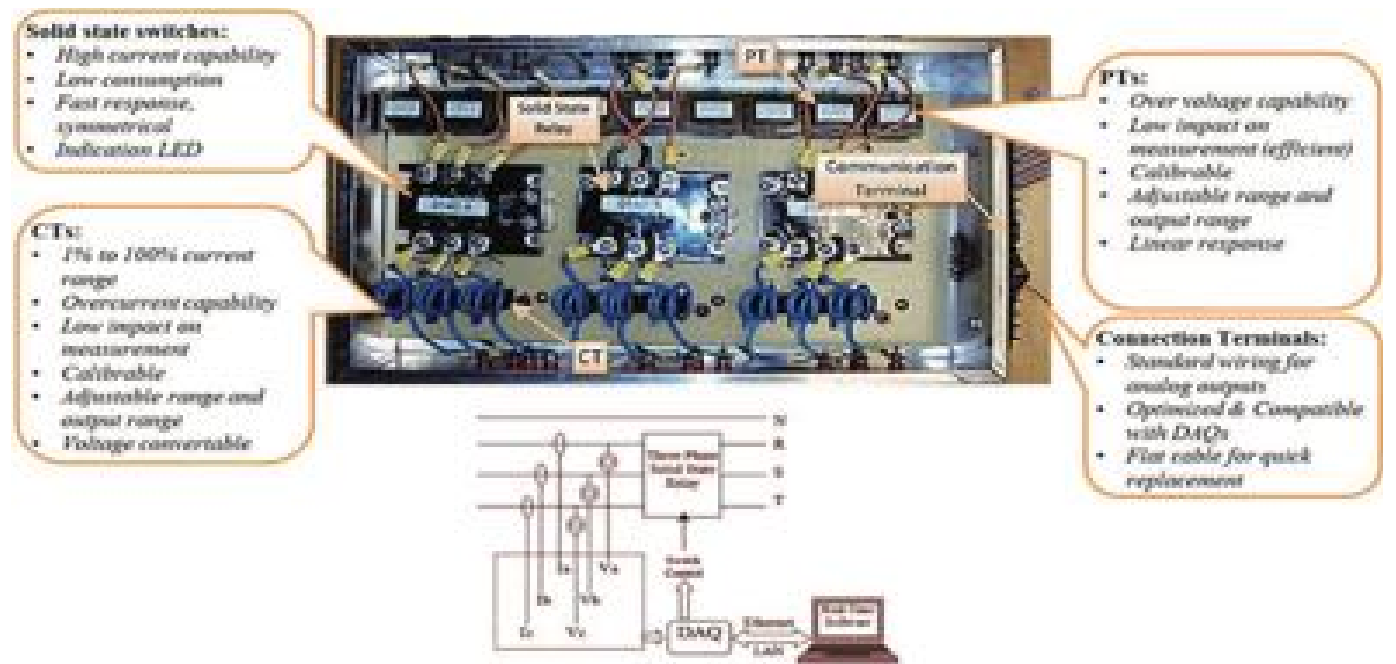

Figure 10.10 Bus models.

\subsubsection{DC/DC Converter}

A PCB based DC-DC boost converter is designed for the laboratory scaled PV emulator. The DC-DC boost converter hardware is shown in Figure 10.11. It contained an 
SK45GB063 IGBT module, and the power rating is $3 \mathrm{~kW}$. The capacity of the capacitor on the high voltage side is $3600 \mathrm{uF}$, and the inductance of the coupled inductor is $3 \mathrm{mH}$. This DC-DC converter is operated with a $5 \mathrm{kHz}$ switching frequency.

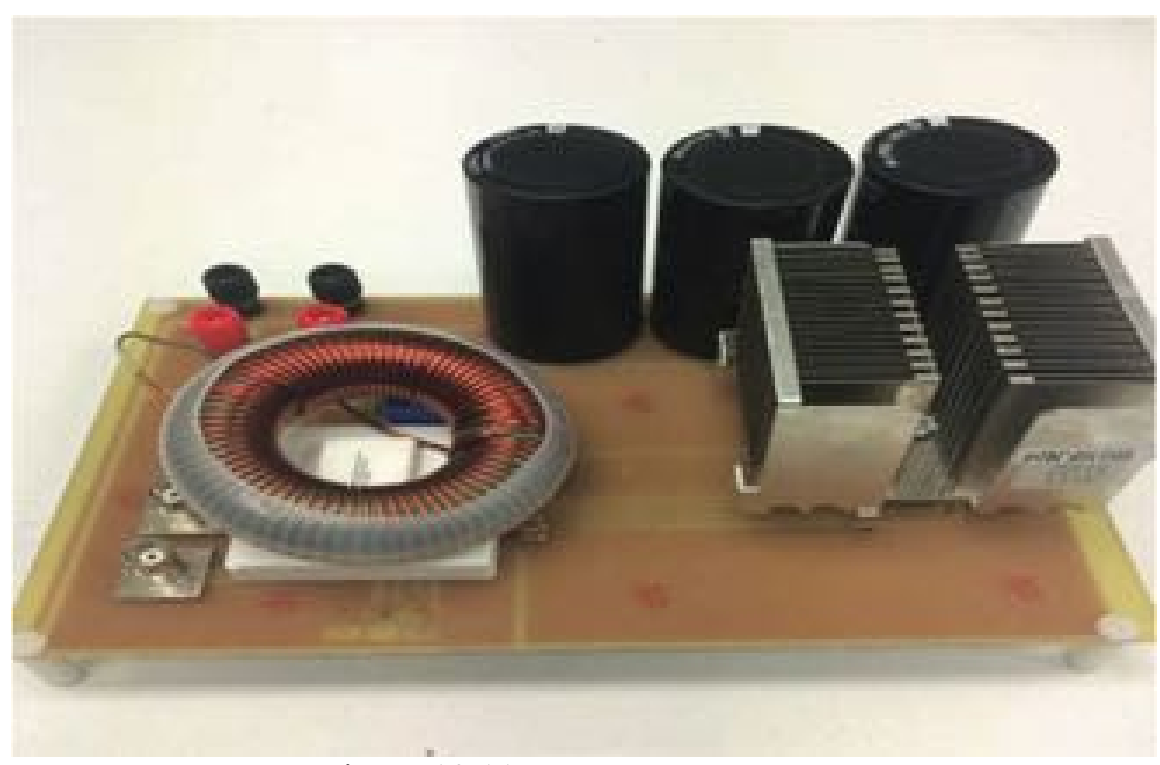

Figure 10.11 DC-DC Boost Converter.

\subsubsection{DC/AC Converter}

The bi-directional AC/DC converter can take control of the AC side frequency and voltage amplitude. The DC bus voltage is regulated by controlling the charging and discharging of the battery banks, which also means controlling the current flow through the bidirectional DC/DC converter. The DSP-based embedded dSpace control platform is used to control the inverter-based DER model. The DC/AC IGBT-based converter is shown in Figure 10.12. 


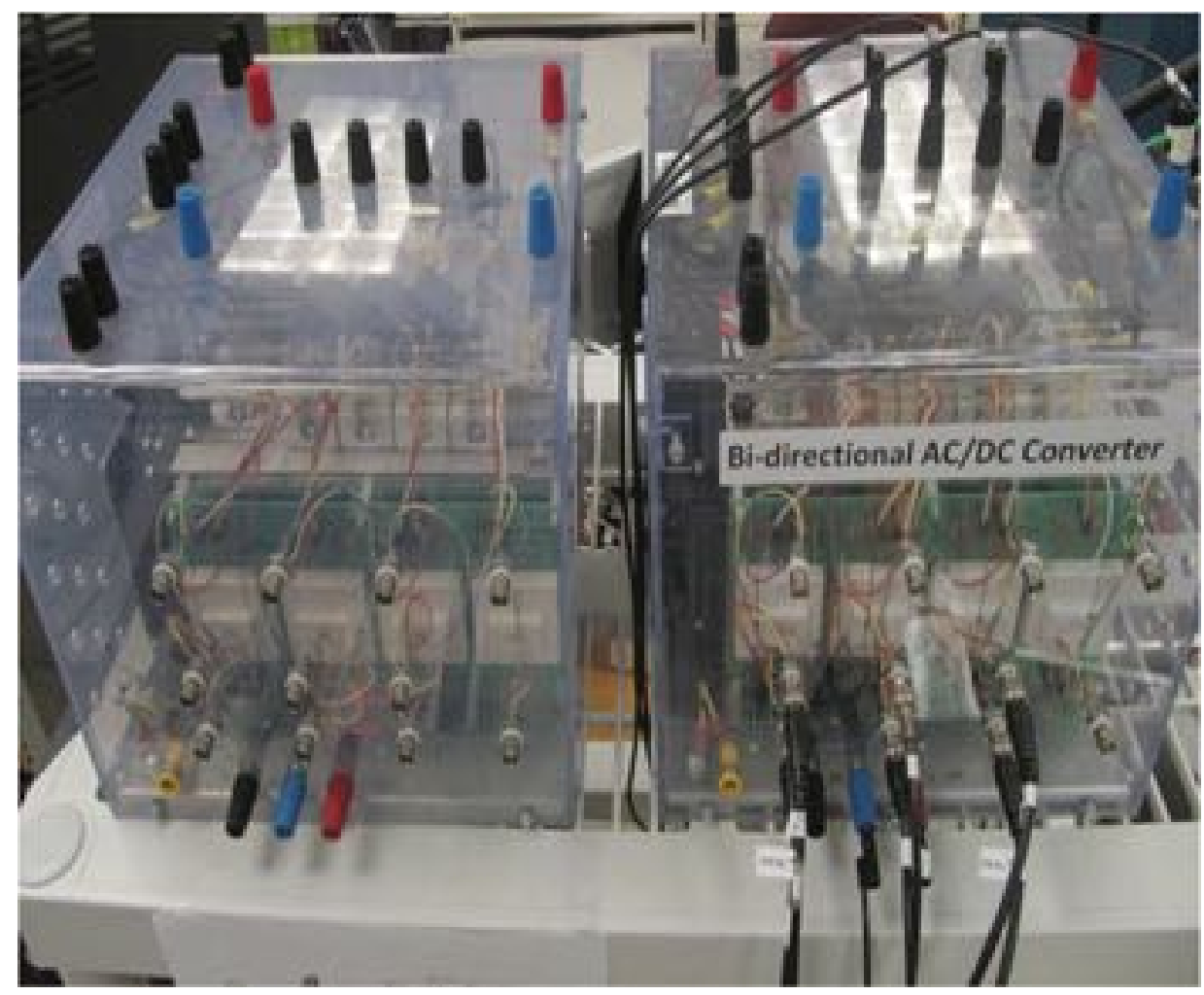

Figure 10.12 The DC/AC IGBT-based converter.

\subsection{Hybrid DC-AC operation and experiment's guideline steps}

\subsubsection{Hybrid DC-AC grid operation}

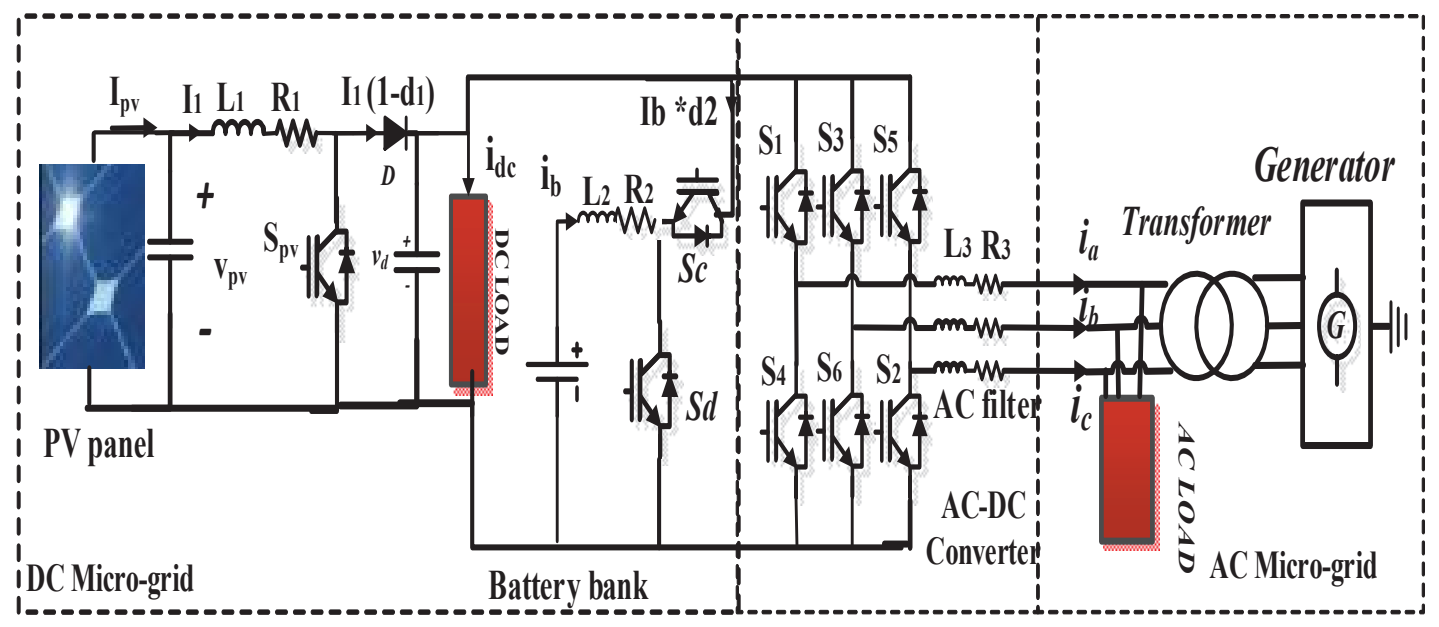

Figure 10.13 The hybrid AC/DC microgrid. 
Figure 10.13 shows the electric circuit of the DC-AC inverter that is connected to the main grid. Considering regular operational mode, the DC-AC inverter's main duty is to maintain the DC bus voltage level within desirable limits. This is achieved via proper regulation of the flowing direct current that establish the operation of the DC part of the grid as a large and pure resistive load at unity power factor, on the other hand, considering islanding mode, the slack generator that is located in the AC side of the hybrid microgrid with the DC-AC inverter operating at current control mode. Utilizing the battery bank state of charge (SOCs), we deploy an aggregator to oversee the transfer power signals through the bidirectional converter with a task to regulate the flow of energy between the two sides of the hybrid microgrid. It should be noted that not accounting for the slack generator at the AC side due to any potential faults or misoperation, and the converter assumes the responsibility to manage the hybrid grid in what is known as frequency regulation mode in order to help the AC side regulate its frequency limit and voltage levels with the absence of the slack generator.

In order to validate our proposed control methodology for the operation and energy management of the hybrid microgrid, we perform our study using hardware-in-the-loop implementation to provide experimental verification to our work. This hardware testbed is scaled down to ensure better accuracy and representation of the results. Figure 10.14. shows the hardware configuration of the testbed.

The system contains two induction generators, labeled G1 and G2. The first generator is attached to bus 1 and considered as the system's slack generator that has the mission of regulating the frequency of the AC side of the hybrid grid. On the other hand, 


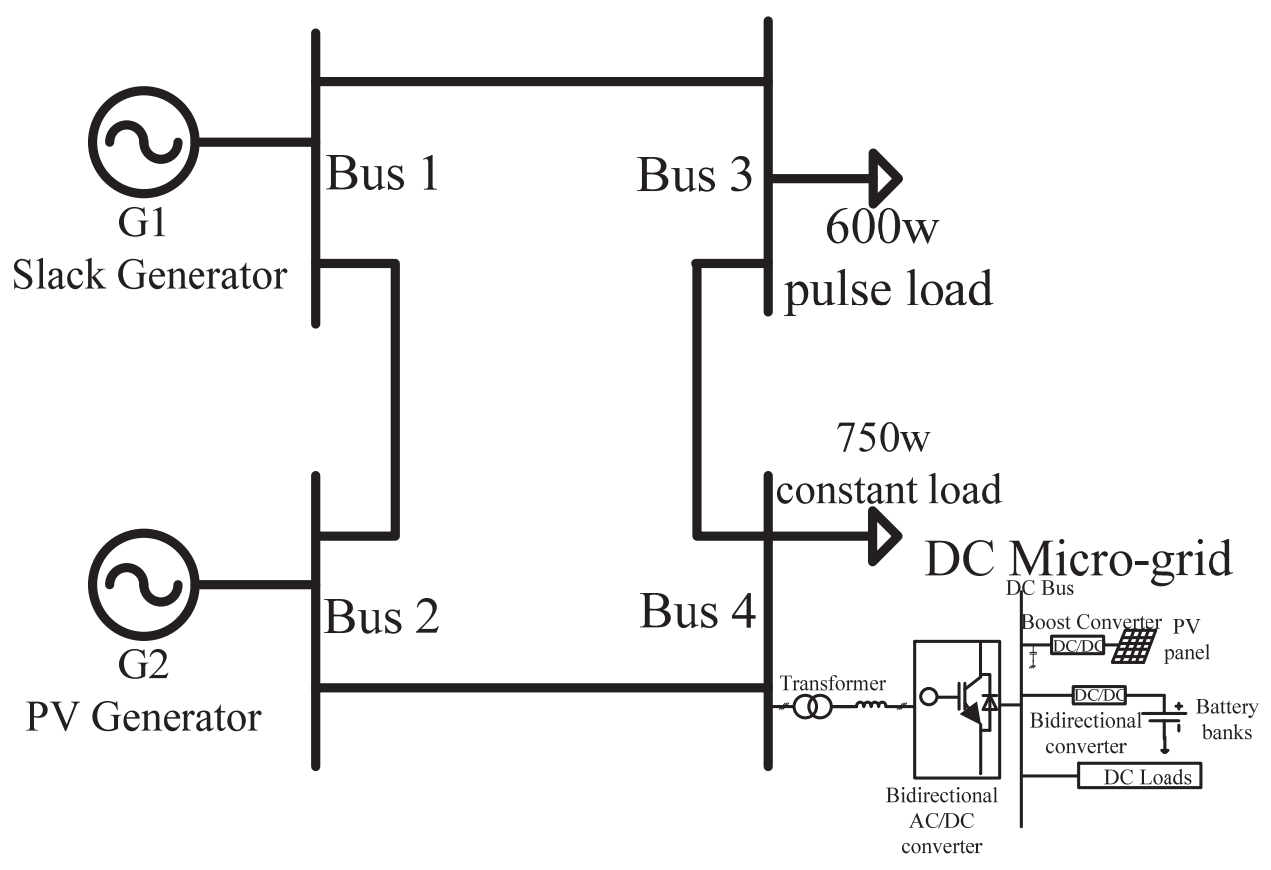

Figure 10.14 The hybrid AC/DC microgrid.

the second generator, as seen from the figure, is connected to bus 2 operating as a voltageregulator bus, which yields only constant power. A $750 \mathrm{~W}$ constant load is attached at bus 3, with another $600 \mathrm{~W}$ pulsed load attached at bus 4 . Moving away from the AC to the DC side of the hybrid grid, an interlinking bidirectional converter is connected to ensure proper and safe sharing of power between the two sides. One of the aspects that the established test bed allowed us to measure is the level of contribution the DC part could provide to the operation of the $\mathrm{AC}$ side of the hybrid grid as well as to the overall hybrid grid performance as well. Specifically, we presented in one of this dissertation's work, namely the vectordecouple control algorithm presented in chapter 2, the methodology of how can the DC side of the grid is used in an effective manner to regulate the frequency of the AC side while maintaining a secured within limits voltage levels at the hybrid microgrid as a whole. The vector-decoupled control allows us to recognize the kind of frequency deficiency in 
the system operation and indicate whether it comes from the slack generator disconnection from the microgrid, as shown in Figure 10.15. (a) presents the level of power received by the microgrid from its two synchronous generators during such a case. Figure 10.15. (b) show the frequency variation as a result of sudden increase of pulsed loads on the system.

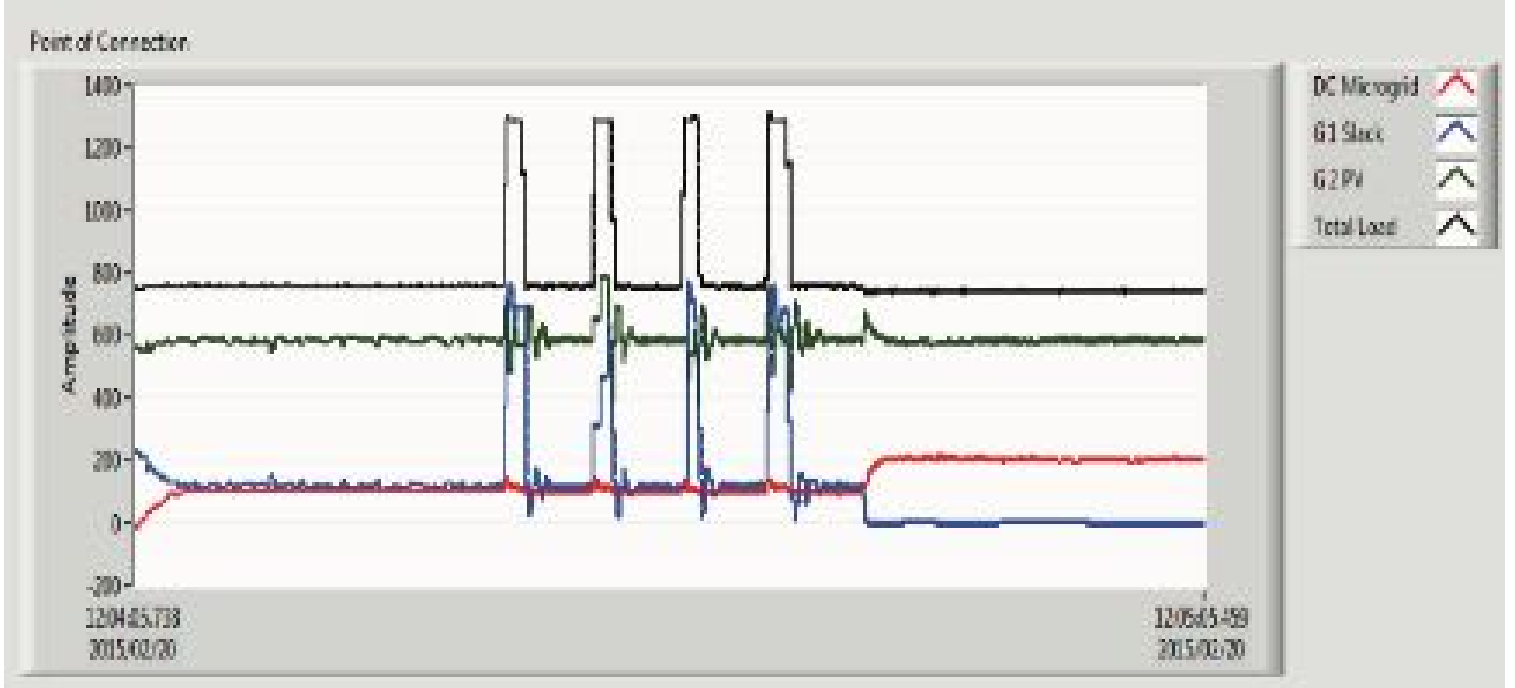

(a)

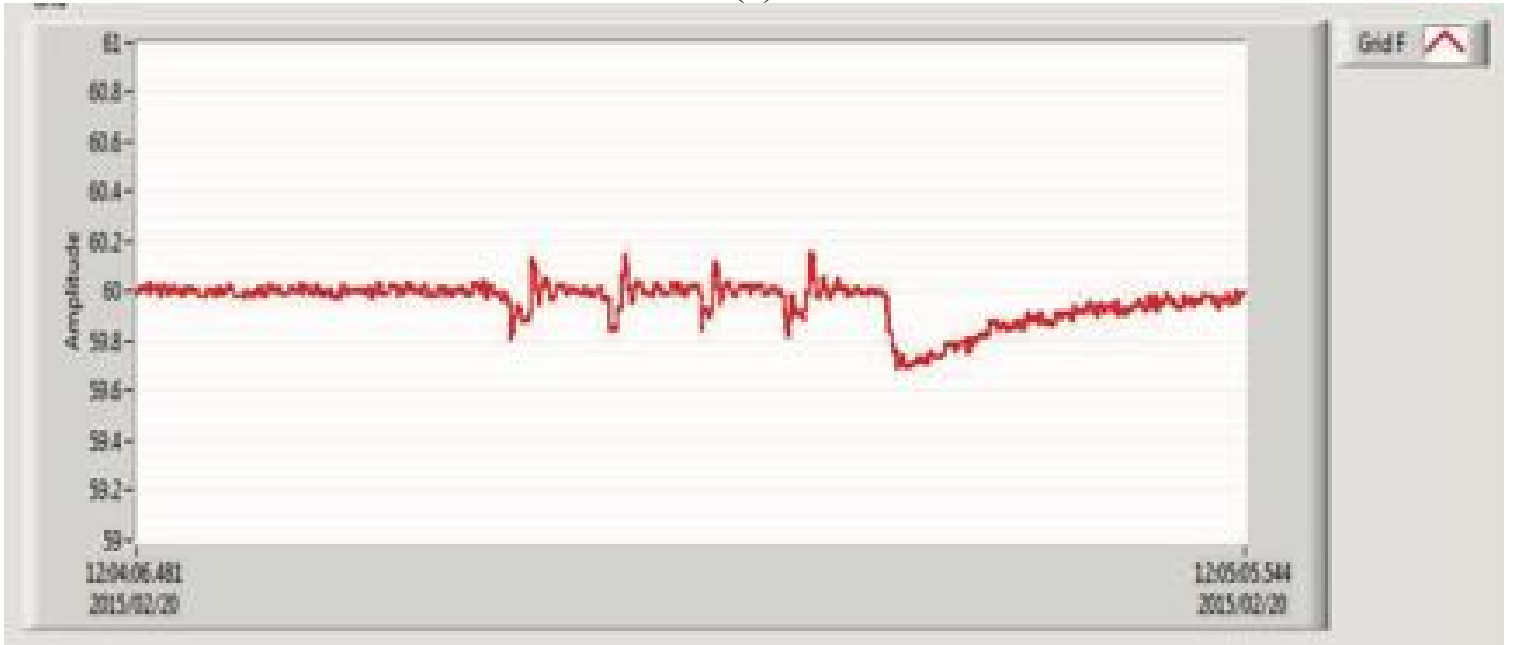

(b)

Figure 10.15 Hybrid microgrid operation performance under the severe condition: a) Correspodnet system frequency, b) power received at the microgrid. 


\subsubsection{Experiment's guideline steps}

\subsubsection{AC side operation guide}

\section{DESCRIPTION:}

- It has four generators, automatic synchronizer, loads, and measurements (data acquisition).

- Labview controls the system operation.

- There is an emergency switch in front of each desk for the communication. If some switches get stuck and not responding, we can use this switch. Push it and then push again to restore to its original position.

- There is an emergency switch for the whole testbed beside the door.

\section{LOCATIONS:}

- On nain testbed computer.

- Use testbed account with password: powerlab.

- $\quad$ On the desktop,>> Open testbed2016 LabVIEW file on the desktop.

\section{OPERATION:}

\section{1- Check the components:}

- Check all the components first (checking the microcontroller) using (self-check)

$\circ \quad$ Tool menu $>>$ measurements and automation explorer

$\circ$ In automatic explorer window $>>$ click the left arrow (expand) of Device and interface $>>$ network devices $>>$ right-click $(\mathrm{RC})$ on NICDAQ$9188($ CDAQ9188.vi) $>>$ self-test. 
On top of the window, a message appears that the test is successful unless there will be a problem.

- Make a self-test for the upper three files (Dev1, Dev2, and Dev3) by RC and self-test.

- Then close the checking window.

Hint:

- In the main window (testbed2016.vi), there are four main windows:

- DAQ window: It shows all the measurements.

○ SCADA window: it shows the switches of all the generators, lines, and loads.

○ Generator control window: for generators and loads manual control.

○ Dynamic brake window: for synchronization.

- Each window you need to make it RUN from the button on the left top of the page (RUN), and the dynamic brake make it (continuously RUN).

\section{2- Show the SCADA window:}

- Double click on SCADA.vi.

\section{3- Show the DAQ window:}

- Double click on DAQ.vi and press RUN on the DAQ window. 
- Show all the figures using a visible graph switch in the middle of the window, but close it as it consumes memory space.

- Check all the measurements to be logical.

\section{4- Running the first generator (G1) as a slack generator and loading it with L1 and}

\section{L2.}

- $\quad$ Turn the generator ON from the station manually.

- Double click on the generator control.vi to open the generator control window.

- In generator control. Vi window, we can see the following for each generator.
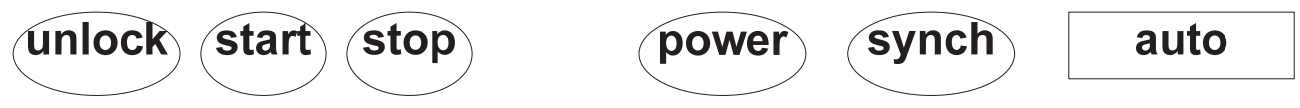

Unlock: This means sending the password to the generator driver to be able to receive any command.

Start: means running the associated generator.

Stop: means OFF the associated generator.

Power: to show the measurements of the generator.

Synch: to show the synchronization window

Auto: it can be used for automatic synchronization (it will try only).

Manual: is used for synchronizing the generator with the bus manually (very important). 


\section{a. Running generator steps:}

- In generator control. Vi window.

- Run the window from the RUN button.

○ Press unlock G1 and put G1 speed $=\mathbf{6 0 0}(60 \mathrm{~Hz}))$.

- Check the command status at the bottom of the same window: the command and the response box need to be the same, if not try to press the switch again unless there will be a communication problem.

○ Press G1 start, you suppose to hear the sound of G1, if not try to increase 600 by one step from the left switch and then put 600 again.

o Press manual, to connect G1 with the bus.

- Check the synchronizer switch to be red (the four switches on the right of the dynamic brake).

\section{b. Feeding the first load steps:}

- In the SCADA window, switch all the switches in the bath from G1 to L1. Moreover, check the response of each switch physically by the red light.

○ Then increase the first load L1 step by step (each step represents $300 \mathrm{~W}$ ).

- Increase it until $900 \mathrm{~W}$ and see the current and the power increase in the measurements. 


\section{c. Feeding the second load steps:}

- In the SCADA window, switch all the switches in the bath from $\mathbf{G 1}$ to $\mathbf{L 2}$. and check the response of each switch physically by the red light.

○ Then increase the first load $\mathbf{L} 2$ step by step (each step represents $300 \mathrm{~W}$ ).

Increase it until $900 \mathrm{~W}$ and see the current and the power increase in the measurements.

\section{5- Synchronizing G2 with G1:}

- The synchronization will be done using the dynamic brake (variable resistance).

- $\quad$ Feed each load L1 and L2 by $\mathbf{6 0 0} \mathbf{W}$, in order to make the load big enough to make G2 loaded after synchronization directly.

- Unlock G2.

- Set the G2 torque reference to 21 N.m, which is suitable for synchronization.

- Press G2 start, you suppose to hear it is sound, if not increase the torque by one step and decrease it again.

- Wait until the frequency of G2 reaches $62 \mathrm{~Hz}$ which is the maximum of the driver.

- Open the synchronization window G2 by pressing the synch button on the generator control window.

- Go to dynamic brak.vi window.

- Press RUN continuously to run the window.

- $\quad$ Click G2. 
- By observing the synchronization window, you can see the voltage of the bus and the generator. The difference between these voltages need to be small (perfect 122 $\mathrm{V}$ and $117 \mathrm{~V})$.

- If the difference is significant, you can decrease it by two ways:

○ Decreasing the load L2 manually.

- Increasing the $\mathrm{G} 2$ torque.

- Keep watching the synchronization window, until the two long bars become full of green.

- Then, press the manual of G2. You can see how hard was the synchronization from the power figures.

- Once the synchronization is done:

○ Off dynamic brake.

○ Put $\mathbf{G 2}$ torque $\mathbf{= 2 3}$.

- Increase manual load by one step.

○ Put $\mathbf{G 2}$ torque $\mathbf{= 2 5}$.

- Increase the manual load by another step.

- Close the dynamic brake window.

- If the synchronization takes a long time, try to decrease the manual loads.

\section{6- Connecting to the DC microgrid setup}

- Check that the DC setup is ready with Idref $=3$.

- Close the switch $\mathbf{5 0 C}$.

- After synchronization, makes $I_{\text {dref }}=5$. 
7- Islanding mode: means disconnecting G1 and making G2 and microgrid working properly without frequency drop, by applying V2G mode on the inverter control.

- Increase torque reference of G2 until the power coming from G1 to be less than $300 \mathrm{~W}$.

- Check that the microgrid has a DC load connected to the DC bus.

- Open 320C or 380A switches to isolate G1.

- Once the switch is opened, activate V2G mode with ref frequency $60 \mathrm{~Hz}$.

\section{8- Shut down the system}

- Decrease the power flow between G1 and G2 by decreasing L2 or increasing G2 torque.

- Make 380A switch OFF.

- $\quad$ Stop G2.

- Press manual switch of G2.

- Stop G1.

- Press manual switch of G1.

- Put all loads at 0 , then close all loads switches.

- Close all windows.

- Turn off the generators manually from the station. 


\subsubsection{DC side operation guide}

\section{DESCRIPTION:}

- It has DC supply, and DC loads are connected directly to the DC bus.

- The DC bus is connected to the AC side through the three-phase inverter, and the controller is implemented on DSPACE.

- A three-phase transformer is used between the inverter and the grid (50-208 V).

- The inverter controller can work in two modes:

$\circ$ The energy transfer mode in which the DC microgrid can exchange the active power with the AC side, using manually sit $\mathrm{I}_{\text {dref. }}$ In this mode, the PWM switch controls the synchronization and needs to be 0 .

- When Idref is negative, this means injecting power to the ac side and the positive means of absorbing power from the $\mathrm{AC}$ side. It ranges from 2 to 5.

- The frequency regulation mode: In which the reference frequency is given, and the system changes the power transfer to get the required frequency in islanding mode. In this mode V2G on a need to be active.

\section{LOCATIONS:}

- $\quad$ The inverter control folder exists on Desktop $>>$ energy conversion project $>>$ AC_to_DC_V5 (simulink file).

- The layout file (for the control desk) exists in the same folder with the same name (AC_to_DC_V5). 


\section{For active power injection operation:}

- Put the DC supply for $200 \mathrm{~V}$.

- Ask hazar to connect his setup (close switch 50C on SCADA window).

- Put Idref to -3 (5 means transferring about 300 watts and this is the max) and then increase it to 5 .

- $\quad$ Put the PWM button on 0 .

\section{For frequency regulation operation:}

- Put the DC supply for $200 \mathrm{~V}$.

- Connect the Microgrid bus with the AC voltage (close switch 50C on Labview).

- Decrease Idref to 3 such that the power coming from the DC side to be almost $200 \mathrm{~W}$.

- Check the microgrid ON button. 


\section{Chapter 11 Conclusions and Recommendations for Future Work}

\subsection{Conclusions}

Demand-side management has a vital role in supporting the demand rise in smart grid infrastructure. Since the innovations at different power system infrastructures' levels facilitate the integration of new smart grid ideas. Therefore, these new architectures of the smart grid add an extra burden on the grid regarding complexity and uncertainty. Besides, the increased penetration of renewable energy, Electric Vehicles (EVs), and time-varying loads in the distribution system, the grid will be vulnerable to unusual, challenging experiences for utility-customer interactions. Household loads represent a significant percentage of electrical energy consumption. The households' demand-side response (DSR) enables active participation of these loads in the grid, enhancing power system stability and quality.

This dissertation provided management and control strategies to make the best use of demand-side management in the power systems. The management and control satisfy the customer and the operator preferences, the system technical constraints, and the involved economic issues. In this paradigm, the deployment of smart homes which allows active load participation are appropriate solutions that can reduce the drawbacks of the massive penetration of renewable energy, Electric Vehicles (EVs), and time-varying loads in the distribution system.

The innovations at different power system infrastructures' levels facilitate the integration of new smart grid ideas. However, new architectures of the smart grid add an extra burden on the grid regarding complexity and uncertainty. As a result of the increased 
penetration of renewable energy, Electric Vehicles (EVs), and time-varying loads in the distribution system, the grid will be vulnerable to unusual, challenging experiences for utility-customer interactions. Household loads represent a significant percentage of electrical energy consumption. The households' demand-side response (DSR) enables active participation of these loads in the grid, enhancing power system stability.

Consequently, the forecasting of household energy consumption is crucial for household DSR programs. Precise short-term load forecasting (STLF) has a significant effect on the accuracy of the household DSR. However, STLF is challenging at this level of the grid due to uncertainty and volatility in load consumption originating from customer behavior, which is too stochastic to predict.

Firstly, I developed an innovative methodology to enhance household demand forecasting based on energy disaggregation for Short Term Load Forecasting. This approach is constructed from Feed-Forward Artificial Neural Network forecaster and a preprocessing stage of energy disaggregation. This disaggregation technique extracts the individual appliances' load demand profile from the aggregated household load demand to increase the training data window for the proposed forecaster. These proposed algorithms include two benchmark disaggregation algorithms; Factorial Hidden Markov Model (FHMM), Combinatorial Optimization in addition to three adopted Deep Neural Network, long short- term memory (LSTM), Denoising Autoencoder, and a network which regress start time, end time, and average power. The proposed load forecasting approach outperformed the currently available state-of-the-art techniques, namely, root mean square 
error (RMSE), normalized root mean square error (NRMSE), and mean absolute error (MAE).

Also, the real-time operation of the energy management system (RT-EMS) is one of the vital functions of Microgrids (MG). In this context, the reliability and smooth operation should be maintained in real-time regardless of load and generation variations and without losing the optimum operation cost.

In this dissertation, I developed a real-time operation energy management system (RTEMS) at the residential Microgrid level. This work presents a design and implementation of an RT-EMS based on Multiagent system (MAS) and the fast converging developed algorithm to minimize the MG operational cost and maximize the real-time response in grid-connected MG. The RT-EMS has the main function to ensure the energy dispatch between the distributed generation (DG) units that consist of this work of a wind generator, solar energy, energy storage units, controllable loads, and the main grid. The MAS has features such as peer-to-peer communication capability, a fault-tolerance structure, and high flexibility, which make it convenient for the MG context. Each component of the MG has its managing agent. While the $\mathrm{MG}$ operator (MGO) is the agent responsible for running to ensure the seamless operation of the MG in real-time, the MG supervisor (MGS) is the agent that intercepts sudden high load variations and computes the new operating point.

In addition, the proposed RT-EMS develops integration of the MAS platform with the Data Distribution Service (DDS) as a middleware to communicate with the physical units. In this work, the proposed algorithm minimizes the cost function of the MG as well as maximizes the use of renewable energy generation; then, it assigns the power reference to 
each DG of the MG. Experimental validation in an MG testbed was conducted to verify the performance of the proposed system. Results show the reliability and effectiveness of the proposed multiagent based RT-EMS. Various scenarios were tested, such as normal operation as well as sudden load variation.

Also, the microgrids offer an essential solution to enable a resilient grid infrastructure since they can continue operating in case of a utility outage. The futuristic distribution system, which is one of the fundamental smart grid concepts, can be viewed as a cluster of microgrids, and each microgrid can be considered to be a cluster of distributed energy resources DERs. Microgrids mostly depend on intermittent renewable resources. This configuration may introduce severe stability problems, especially during the islanded operation. Therefore, I proposed a hybrid residential microgrid system for future city houses. It is composed of both AC and DC networks tied together through an interlinking bidirectional AC/DC converter (IC). The DC network contains the DC loads and the distributed energy sources (DES) such as Photovoltaic (PV) and battery storage. The AC network includes the AC loads and a synchronous AC generator as the only AC source in islanding operation in this system. The islanding operation at this level of the grid, prone the network to be more vulnerable to stability issues. Therefore, it is essential to obtain the best control parameters for the IC controller, which ensures sufficient power exchange and stable operation between the DC/AC networks.

Consequently, a Genetic Algorithm (GA) has been used to tune the vector decoupling control parameters of the IC controller to find the best parameter combination to improve the hybrid grid performance. The proposed scheme maintains system stability during 
various conditions such as variable or pulsed demand and uncertain renewable powersharing. The tuned vector decoupling controlled sinusoidal pulse width modulation (SPWM) technique has been used to not only allow the IC to maintain stable voltage and frequency but also tracks the active and reactive power setpoints.

In addition, I developed a control methodology to facilitate the smart integration of a DC Microgrid to Neighborhood Low Voltage Distribution Network (NLVDN). The DC Microgrid connected to the NLVDN through a three-phase voltage source inverter (VSI). In which, the VSI works as Distribution Static Compensator (DSTATCOM), and the DC link provides an integration point for the PV and the DC native loads. The proposed technique is capable of compensating reactive power, unbalance, and harmonics demanded by three-phase non-linear loads and unbalanced connected to the distribution side improves the power quality. Besides, it can prevent the source from getting overloaded by providing active power support to the load.

\subsection{Recommendations for Future Work}

This dissertation covered several aspects related to the management and control strategies to make the best use of demand-side management in the power systems. The provided solutions in this dissertation are critical because it offers valuable answers for many issues that are related to the active participation of the load consumption sector in power grids stability. Moreover, the studies provide a practical energy management framework and control algorithm that can lead to the optimal control and operation of individual customers in the distribution level and thus bringing benefits to the environment, customers, and system operators. 
Despite the provided solutions in this dissertation, the design of a smart grid as an integrated cyber-physical system is needed. Several issues related to system modeling, scalability, interoperability, and security need to be covered in future research. In addition, developing anomaly detection for smart grid monitoring and control systems that utilize both cybersecurity rules and physical system characteristics is needed. This research should also address the interoperability and system expandability challenges in modern energy systems by working on a unified data model and utilization of a data-centric approach.

Another issue that needs to be adequately addressed is the interaction of the stochastic natures of EVs, renewable energies, especially wind and solar energy, with the energy storage and the conventional unit commitment. Due to the stochastic nature of renewable energies and EVs, old traditional methods for an optimal unit commitment should be readapted to consider these stochastic entities to ensure the optimal and reliable operation of the power grid.

Finally, a very pressing problem that needs to be investigated and analyzed thoroughly is the modeling of the different kinds of attacks on the power system, and more importantly, is how the power system can be controlled to mitigate or reduce the damage of these attacks. 


\section{List of References}

[1] K. L. López, C. Gagné and M. A. Gardner, "Demand-Side Management using Deep Learning for Smart Charging of Electric Vehicles," in IEEE Transactions on Smart Grid.

[2] J. Flottemesch and M. Rother, "Optimized energy exchange in primary distribution networks with DC links," 2004 IEEE International Conference on Electric Utility Deregulation, Restructuring and Power Technologies. Proceedings, 2004, pp. 108116 Vol.1.

[3] Marino, D.L.; Amarasinghe, K.; Manic, M. Building energy load forecasting using Deep Neural Networks. In Proceedings of the IECON 2016-42nd Annual Conference of the IEEE Industrial Electronics Society, Florence, Italy, 24-27 October 2016; pp. 7046-7051.

[4] Shi, H.; Xu, M.; Li, R. Deep Learning for Household Load Forecasting - A Novel Pooling Deep RNN. IEEE Trans. Smart Grid 2017.

[5] H. Shi, M. Xu, and R. Li, "Deep Learning for Household Load Forecasting; A Novel Pooling Deep RNN," IEEE Trans. Smart Grid, vol. PP, no. 99, pp. 1-1, 2017.

[6] F. Ebrahim and O. Mohammed, "Household Load Forecasting Based on a PreProcessing Non-Intrusive Load Monitoring Techniques," 2018 IEEE Green Technologies Conference (GreenTech), Austin, TX, 2018, pp. 107-114.

[7] George. W. Hart, "Nonintrusive appliance load monitoring," Proc. IEEE, vol. 80, no. 12, pp. 1870-1891, Dec. 1992.

[8] George. W. Hart, "Residential energy monitoring and computerized surveillance via utility power flows," IEEE Technol. Soc. Mag., vol. 8, no.2, pp. 12-16, Jun. 1989.

[9] Sung. W. Park, Lee. B. Baker and Paul. D. Franzon, "Appliance Identification Algorithm for a Non-Intrusive Home Energy Monitor using Cogent Confabulation," in IEEE Transactions on Smart Grid.

[10] Cvetkovic, I.; Boroyevich, D.;Mattavelli, P.; Lee, F.C.; Lucia, O.; Sarnago, H. Design of Home Appliances for a DC-Based Nanogrid System: An Induction Range Study Case. IEEE J. Emerg. Sel. Top. Electron. 2013, 1, 315-326.

[11] Parchure, A.; Tyler, S.J.; Peskin, M.A.; Rahimi, K.; Broadwater, R.P.; Dilek, M. Investigating PV Generation Induced Voltage Volatility for Customers Sharing a Distribution Service Transformer. IEEE Trans. Ind. Appl. 2017, 53, 71-79.

[12] Chamana, M.; Chowdhury, B.H.; Jahanbakhsh, F. Distributed Control of Voltage Regulating Devices in the Presence of High PV Penetration to Mitigate Ramp-Rate Issues. IEEE Trans. Smart Grid 2018, 9, 1086-1095.

[13] Wang, Y.; Zhang, N.; Li, H.; Yang, J.; Kang, C. Linear three-phase power flow for unbalanced active distribution networks with PV nodes. CSEE J. Power Energy Syst. 2017, 3, 321-324. 
[14] Alam,M.J.E.;Muttaqi,K.M.; Sutanto,D.Community Energy Storage forNeutralVoltage RiseMitigation in Four-Wire Multigrounded LV Feeders with Unbalanced Solar PV Allocation. IEEE Trans. Smart Grid 2015, 6, 2845-2855.

[15] Nallusamy, S.; Parvathyshankar, D.; Velayutham, D.; Govindarajan, U. Power quality improvement in a low-voltage DC ceiling grid powered system. IET Power Electron. 2015, 8, 1902-1911.

[16] Wunder, B.; Ott, L.; Szpek, M.; Boeke, U.; Weiß, R. Energy e_cient DC-grids for commercial buildings. In Proceedings of the 2014 IEEE 36th International Telecommunications Energy Conference (INTELEC),Sao Paulo, Brazil, 17-20 August 2014; pp. 1-8.

[17] Peña-Alzola, R.; Bianchi, M.A.; Ordonez, M. Control Design of a PFC with Harmonic Mitigation Function for Small Hybrid AC/DC Buildings. IEEE Trans. Power Electron. 2016, 31, 6607-6620.

[18] Nilsson, D.; Sannino, A. E_ciency analysis of low-and medium-voltage DC distribution systems. In Proceedings of the Power Engineering Society General Meeting, Denver, CO, USA, 6-10 June 2004; pp. 2315-2321.

[19] Arrillaga, J.;Watson, N.R. Power System Harmonics; John Wiley and Sons: Hoboken, NJ, USA, 2004.

[20] Ahmed, F.; Ebrahim, T.; Youssef, A.; Mohammed, O.A. Power Quality Improvements for Integration of Hybrid AC/DC Nanogrids to Power Systems. In Proceedings of the 2017 Ninth Annual IEEE Green Technologies Conference (GreenTech), Denver, CO, USA, 29-31 March 2017; pp. 171-176.

[21] Kraja ci'c, G.; Dui'c, N.; Vujanovi'c, M.; K1lk1,s, ,S.; Rosen, M.A.; Al-Nimr, M.A. Sustainable development of energy, water, and environmental systems for future energy technologies and concepts. Energy Convers. Manag. 2016, 125, 1-14.

[22] Chowdhury, B.H. Power Quality. IEEE Potentials 2001, 20, 5-11.

[23] Munir, S.; Li, Y.W. Residential Distribution System Harmonic Compensation Using PV Interfacing Inverter. IEEE Trans. Smart Grid 2013, 4, 816-827.

[24] Wada, K.; Fujita, H.; Akagi, H. Considerations of an active shunt filter based on voltage detection for installation on a long distribution feeder. IEEE Trans. Ind. Appl. 2002, 38, 1123-1130.

[25] Lee, T.-L.; Cheng, P.-T.; Akagi, H.; Fujita, H. A Dynamic Tuning Method for Distributed Active Filter Systems. IEEE Trans. Ind. Appl. 2008, 44, 612-623.

[26] Cheng, P.-T.; Lee, T.-L. Distributed Active Filter Systems (DAFSs): A New Approach to Power System Harmonics. IEEE Trans. Ind. Appl. 2006, 42, 13011309.

[27] Ward, D.J. The impact of distribution system design on harmonic limits. In Proceedings of the Power Engineering Society 1999 Winter Meeting, New York, NY, USA, 31 January-4 February 1999; Volume 2, pp. 1110-1114.

[28] Haidar,A.M.A.;Muttaqi, K.M.; Sutanto,D. Technical challenges for electric power industries due to grid-integrated electric vehicles in low voltage distributions: A review. Energy Convers. Manag. 2014, 86, 689-700. 
[29] López-Martín, V.M.; Azcondo, F.J.; Pigazo, A. Power Quality Enhancement in Residential Smart Grids Through Power Factor Correction Stages. IEEE Trans. Ind. Electron. 2018, 65, 8553-8564.

[30] Illindala, M.; Venkataramanan, G. Frequency/Sequence Selective Filters for Power Quality Improvement in a Microgrid. IEEE Trans. Smart Grid 2012, 3, 2039-2047.

[31] Corasaniti, V.F.; Barbieri, M.B.; Arnera, P.L. Compensación con filtro activo de potencia hibrido en una planta industrial. In Proceedings of the ARGENCON Congreso Bienal de IEEE Argentina, Córdoba, Argentina, 13-15 June 2012.

[32] Bhattacharya, A.; Chakraborty, C.; Bhattacharya, S. Parallel-Connected Shunt Hybrid Active Power Filters Operating at Different Switching Frequencies for Improved Performance. IEEE Trans. Ind. Electron. 2012, 59, 4007-4019.

[33] Al Sayari, N.; Chilipi, R.; Barara, M. An adaptive control algorithm for gridinterfacing inverters in renewable energy based distributed generation systems. Energy Convers. Manag. 2016, 111, 443-452.

[34] Hamid, M.I.; Jusoh, A. Reduction of waveform distortion in grid-injection current from single-phase utility interactive PV-inverter. Energy Convers. Manag. 2014, 85, 212-226.

[35] Guerrero-Rodríguez, N.F.; Rey-Boué, A.B. Modelling, simulation and experimental verification for renewable agents connected to a distorted utility grid using a RealTime Digital Simulation Platform. Energy Convers. Manag. 2014, 84, 108-121.

[36] Rahman, M.S.; Oo, A.M.T. Distributed multi-agent based coordinated power management and control strategy for microgrids with distributed energy resources. Energy Convers. Manag. 2017, 139, 20-32.

[37] Wu, J.-C.; Wu, K.-D.; Jou, H.-L.; Wu, Z.-H.; Chang, S.-K. Novel power electronic interface for grid-connected fuel cell power generation system. Energy Convers. Manag. 2013, 71, 227-234.

[38] Calleja, H.; Jimenez, H. Performance of a grid-connected PV system used as an active filter. Energy Convers. Manag. 2004, 45, 2417-2428.

[39] Altin, N.; Ozdemir, S. Three-phase three-level grid interactive inverter with fuzzy logic based maximum power point tracking controller. Energy Convers. Manag. 2013, 69, 17-26.

[40] the J. Torriti, "Price-based demand side management: Assessing the impacts of timeof-use tariffs on residential electricity demand and peak shifting in Northern Italy," Energy, vol. 44, no. 1, pp. 576-583, Aug. 2012.

[41] P. Samadi, H. Mohsenian-Rad, V. W. S. Wong, and R. Schober, "Tackling the load uncertainty challenges for energy consumption scheduling in smart grid," IEEE Trans. Smart Grid, vol. 4, no. 2, pp. 1007-1016, Jun. 2013.

[42] B. Hayes, I. Hernando-Gil, A. Collin, G. Harrison, and S. Djoki'c, "Optimal power flow for maximizing network benefits from demand-side management," IEEE Trans. Power Syst., vol. 29, no. 4, pp. 1739-1747, Jul. 2014. 
[43] C. O. Adika and L. Wang, "Demand-side bidding strategy for residential energy management in a smart grid environment," IEEE Trans. Smart Grid, vol. 5, no. 4, pp. 1724-1733, Jul. 2014.

[44] T. Mancini et al., "Demand-aware price policy synthesis and verification services for smart grids," in Proc. IEEE Int. Conf. Smart Grid Commun. (SmartGridComm), Venice, Italy, Nov. 2014, pp. 794-799.

[45] Y. Li, B. L. Ng, M. Trayer, and L. Liu, "Automated residential demand response: Algorithmic implications of pricing models," IEEE Trans. Smart Grid, vol. 3, no. 4, pp. 1712-1721, Dec. 2012.

[46] Y. Ozturk, D. Senthilkumar, S. Kumar, and G. Lee, "An intelligent home energy management system to improve demand response," IEEE Trans. Smart Grid, vol. 4, no. 2, pp. 694-701, Jun. 2013.

[47] Z. Zhao, W. C. Lee, Y. Shin, and K.-B. Song, "An optimal power scheduling method for demand response in-home energy management system," IEEE Trans. Smart Grid, vol. 4, no. 3, pp. 1391-1400, Sep. 2013.

[48] T. Mancini et al., "User flexibility aware price policy synthesis for smart grids," in Proc. IEEE DSD, Funchal, Portugal, 2015, pp. 478-485.

[49] Ahmed. F. Ebrahim, Osama A. Mohammed," Pre-Processing of Energy Demand Disaggregation Based Data Mining Techniques for Household Load Demand Forecasting. Inventions 2018, 3, 45.

[50] Ahmed. F. Ebrahim and O. A. Mohammed, "Energy Disaggregation Based Deep Learning Techniques: A pre-processing Stage to Enhance the Household Load Forecasting," 2018 IEEE Industry Applications Society Annual Meeting (IAS), Portland, OR, USA, 2018, pp. 1-8.

[51] Ahmed. F. Ebrahim and O. Mohammed, "Household Load Forecasting Based on a Pre-Processing Non-Intrusive Load Monitoring Techniques," 2018 IEEE Green Technologies Conference (GreenTech), Austin, TX, 2018, pp. 107-114. DOI: 10.1109/GreenTech.2018.00028

[52] Fatima Zahra Harmouch, Ahmed. F. Ebrahim, Mohammad M. Esfahani, Nissrine Krami, Nabil Hmina, Osama A. Mohammed," An Optimal Energy Management System for Real-Time Operation of Multiagent-Based Microgrids Using a T-Cell Algorithm". Energies 2019, 12 (5), 3004.

[53] Ahmed. F. Ebrahim, A. A. S. Mohamed, A. A. Saad and O. A. Mohammed, "Vector Decoupling Control Design Based on Genetic Algorithm for a Residential Microgrid System for Future City Houses at Islanding Operation," SoutheastCon 2018, St. Petersburg, FL,2018,pp.1-5.doi: 10.1109/SECON.2018.8479013.

[54] Ahmed. F. Ebrahim, Ahmed A. Saad, Osama A. Mohammed," Smart Integration of a DC Microgrid: Enhancing the Power Quality Management of the Neighborhood Low-Voltage Distribution Network. Inventions 2019, 4(2), 25.

[55] Ahmed. F. Ebrahim, Tarek A. Youssef, Osama A. Mohammed, " Power Quality Improvements for Integration of Hybrid AC/DC Nanogrids to Power Systems" IEEE Greentech 2017, Denver, Colorado, held between March 29 - 31, 2017. 
[56] D. E. Olivares et al., "Trends in Microgrid Control," in IEEE Transactions on Smart Grid, vol. 5, no. 4, pp. 1905-1919, July 2014.

[57] S. A. Arefifar, Y. A. R. I. Mohamed and T. H. M. El-Fouly, "Supply-AdequacyBased Optimal Construction of Microgrids in Smart Distribution Systems," in IEEE Transactions on Smart Grid, vol. 3, no.3, pp. 1491-1502, Sept. 2012.

[58] H. F. Habib, T. Youssef, M. H. Cintuglu and O. A. Mohammed, "Multi-Agent-Based Technique for Fault Location, Isolation, and Service Restoration," in IEEE Transactions on Industry Applications, vol. 53, no. 3, pp. 1841-1851, May-June 2017.

[59] H. F. Habib and O. Mohammed, "Decentralized Multi-Agent System for Protection and the Power Restoration Process in Microgrids," 2017 Ninth Annual IEEE Green Technologies Conference (GreenTech), Denver, CO, 2017, pp. 358-364.

[60] D. Wu, F. Tang, T. Dragicevic, J. C. Vasquez and J. M. Guerrero, "A Control Architecture to Coordinate Renewable Energy Sources and Energy Storage Systems in Islanded Microgrids," in IEEE Transactions on Smart Grid, vol. 6, no. 3, pp. 11561166, May 2015.

[61] Z. Wang, B. Chen, J. Wang, M. M. Begovic and C. Chen, "Coordinated Energy Management of Networked Microgrids in Distribution Systems," in IEEE Transactions on Smart Grid, vol. 6, no. 1, pp. 45-53, Jan. 2015.

[62] H. Lotfi; A. Khodaei, "AC Versus DC Microgrid Planning," in IEEE Transactions on Smart Grid, vol.PP, no.99, pp.1-1, 2015, doi: 10.1109/TSG.2015.2457910

[63] T. Dragičević, X. Lu, J. C. Vasquez and J. M. Guerrero, "DC Microgrids—Part II: A Review of Power Architectures, Applications, and Standardization Issues," in IEEE Transactions on Power Electronics, vol. 31, no. 5, pp. 3528-3549, May 2016.

[64] G. AlLee and W. Tschudi, "Edison Redux: 380 Vdc Brings Reliability and Efficiency to Sustainable Data Centers," in IEEE Power and Energy Magazine, vol. 10, no. 6, pp. 50-59, Nov.-Dec. 2012.

[65] Ahmed T. Elsayed, Ahmed A. Mohamed and Osama A. Mohammed, "DC microgrids and distribution systems: An overview," Electric Power Systems Research Journal, 119, February 2015, 407-417.

[66] H. F. Habib, C. R. Lashway and O. A. Mohammed, "A Review of Communication Failure Impacts on Adaptive Microgrid Protection Schemes and the Use of Energy Storage as a Contingency," in IEEE Transactions on Industry Applications, vol. PP, no. 99 , pp. 1-1.

[67] Hany F. Habib, A.A.S. Mohamed, Mohamad El Hariri, Osama A. Mohammed, "Utilizing supercapacitors for resiliency enhancements and adaptive microgrid protection against communication failures," in Electric Power Systems Research, Volume 145, April 2017, Pages 223-233, ISSN 0378-7796, http://doi.org/10.1016/j.epsr.2016.12.027

[68] H. F. Habib, C. R. Lashway and O. A. Mohammed, "On the adaptive protection of microgrids: A review on how to mitigate cyber attacks and communication failures," 
2017 IEEE Industry Applications Society Annual Meeting, Cincinnati, OH, 2017, pp. 1-8.

[69] S. K. Chaudhary, J. M. Guerrero, and R. Teodorescu, "Enhancing the Capacity of the AC Distribution System Using DC Interlinks-A Step Toward Future DC Grid," in IEEE Transactions on Smart Grid, vol. 6, no. 4, pp. 1722-1729, July 2015.5.

[70] S. Bacha, D. Picault, B. Burger, I. Etxeberria-Otadui and J. Martins, "Photovoltaics in Microgrids: An Overview of Grid Integration and Energy Management Aspects," in IEEE Industrial Electronics Magazine, vol. 9, no. 1, pp. 33-46, March 2015.

[71] Ahmed. F. Ebrahim, S. M. W. Ahmed, S. E. Elmasry and O. A. Mohammed, "Implementation of a PV emulator using programmable DC power supply," SoutheastCon 2015, Fort Lauderdale, FL, 2015, pp.1-7.

[72] Ahmed. F. Ebrahim, T. Youssef, S. M. W. Ahmed, S. E. Elmasry and O. A. Mohammed, "Fault detection and compensation for a PV system grid tie inverter," North American Power Symposium (NAPS), 2014, Pullman, WA, 2014, pp. 1-6.

[73] M. A. Farahat, H. M. B. Metwally, Ahmed abd-Elfatah Mohamed, "optimal choice and design of different topologies of the DC-Dc converter used PV system, at different climate condition in Egypt," Renewable Energy, Volume 43, July 2012, Pages 393-402.

[74] A. A. S. Mohamed, A. Berzoy, and O. Mohammed, "Optimized-fuzzy MPPT controller using GA for stand-alone photovoltaic water pumping system," IECON 2014 - 40th Annual Conference of the IEEE Industrial Electronics Society, Dallas, TX, 2014, pp. 2213-2218.

[75] Vladu, Ecaterina Emilia, and Toma Leonida Dragomir. "Controller tuning using genetic algorithms." In Proc of 1st Romanian-Hungarian Joint Symposium on Applied Computational Intelligence, pp. 1-10. 2004.

[76] Korodi, Adrian, Toma L. Dragomir, and Gianina Gabor. "Temperature Control Solution Using Interpolation for a Geothermal Power Plant." IFAC Proceedings Volumes 39, no. 19 (2006): 235-240.

[77] Kouatli, Issam. "Student advising decision to predict student's future GPA based on Genetic Fuzzimetric Technique (GFT)." In Fuzzy Systems (FUZZ-IEEE), 2015 IEEE International Conference on, pp. 1-8. IEEE, 2015.

[78] Ahmed. F. Ebrahim, Tarek A. Youssef, Osama A. Mohammed, "Power Quality Improvements for Integration of Hybrid AC/DC Nanogrids to Power Systems" IEEE Greentech 2017, Denver, Colorado, held between March 29 - 31, 2017.

[79] H. F. Habib, A. O. Hariri, A. ElSayed and O. A. Mohammed, "Deployment of electric vehicles in an adaptive protection technique for riding through cyber attack threats in microgrids," in IEEE International Conference on Environment and Electrical Engineering and 2017 IEEE Industrial and Commercial Power Systems Europe (EEEIC / I\&CPS Europe), Milan, pp. 1-6, 2017.

[80] A. Elsayed, Ahmed. F. Ebrahim, H. Mohammed, and O. A. Mohammed, "Design and implementation of AC/DC active power load emulator," SoutheastCon 2015, Fort Lauderdale, FL, 2015, pp. 1-5. 
[81] H. Habib, M. E. Hariri, A. Elsayed and O. A. Mohammed, "Utilization of Supercapacitors in Protection Schemes for Resiliency against Communication Outages: A Case Study on Size and Cost Optimization," in IEEE Transactions on Industry Applications, vol. PP, no. 99, pp. 1-1.

[82] Elshaer, M., Mohamed, A., \& Mohammed, O. (2010, November). Smart optimal control of DC-DC boost converter in PV systems. In Transmission and Distribution Conference and Exposition: Latin America (T\&D-LA), 2010 IEEE/PES (pp. 403410). IEEE.

[83] Taghvaee, M. H., M. A. M. Radzi, S. M. Moosavain, Hashim Hizam, and M. Hamiruce Marhaban. "A current and future study on non-isolated DC-DC converters for photovoltaic applications." Renewable and sustainable energy reviews 17 (2013): 216-227.

[84] N. Korolko, Z. Sahinoglu, and D. Nikovski, "Modeling and Forecasting Self-Similar Power Load Due to EV Fast Chargers," IEEE Trans. Smart Grid, vol. 7, no. 3, pp.1620-1629, May 2016.

[85] P. Zhang, X. Zhou, P. Pelliccione, and H. Leung, "RBFMLMR:A Multi-Label Metamorphic Relation Prediction Approach Using RBF Neural Network," IEEEAccess, vol. 5, pp. 21791-21805, 2017.

[86] Z. Yun, Z. Quan, S. Caixin, L. Shaolan, L. Yuming, andS. Yang, "RBF Neural Network and ANFIS-BasedShort-Term Load Forecasting Approach in RealTimePrice Environment," IEEE Trans. Power Syst., vol. 23,no. 3, pp. 853-858, Aug. 2008.

[87] W. Kong, Z. Y. Dong, D. J. Hill, F. Luo, and Y. Xu,"Short-Term Residential Load Forecasting based on Resident Behaviour Learning," IEEE Trans. Power Syst.,pp. $1-1,2017$.

[88] H. S. Hippert, C. E. Pedreira, and R. C. Souza, "Neural networks for short-term load forecasting: A review and evaluation," IEEE Trans. Power Syst., vol. 16, no. 1, pp.44-55, 2001.

[89] A. S. Ahmad et al., "A review on applications of ANNand SVM for building electrical energy consumption forecasting," Renew. Sustain. Energy Rev., vol. 33, pp.102-109, May 2014.

[90] T. Teeraratkul, D. O'Neill, and S. Lall, "Shape-Based Approach to Household Electric Load Curve Clustering and Prediction," IEEE Trans. Smart Grid, pp. 1-1, 2017.

[91] A. Ahmad, N. Javaid, M. Guizani, N. Alrajeh, and Z. A.Khan, "An Accurate and Fast Converging Short-TermLoad Forecasting Model for Industrial Applications in aSmart Grid,” IEEE Trans. Ind. Inform., vol. 13, no. 5, pp.2587-2596, Oct. 2017.

[92] A. Ouammi, "Optimal Power Scheduling for a Cooperative Network of Smart Residential Buildings," IEEE Trans. Sustain. Energy, vol. 7, no. 3, pp. 1317-1326, Jul. 2016.

[93] N. Ahmed, M. Levorato, and G.-P. Li, "Residential Consumer-Centric Demand Side Management," IEEETrans. Smart Grid, pp. 1-1, 2017. 
[94] S. Ryu, J. Noh, and H. Kim, "Deep neural network based demand side short-term load forecasting," in 2016 IEEE International Conference on Smart Grid Communications (SmartGridComm), 2016, pp. 308-313.

[95] E. Mocanu et al., "Optimizing the energy exchangebetween the Smart Grid and Building Systems," in 2014 49th International Universities Power Engineering Conference (UPEC), 2014, pp. 1-6.

[96] D. L. Marino, K. Amarasinghe, and M. Manic, "Building energy load forecasting using Deep Neural Networks," inIECON 2016 - 42nd Annual Conference of the IEEE Industrial Electronics Society, 2016, pp. 7046-7051.

[97] H. Shi, M. Xu, and R. Li, "Deep Learning for Household Load Forecasting - A Novel Pooling Deep RNN,” IEEETrans. Smart Grid, pp. 1-1, 2017.

[98] H. Shi, M. Xu, and R. Li, "Deep Learning for Household Load Forecasting \#x2013; A Novel Pooling Deep RNN,"IEEE Trans. Smart Grid, vol. PP, no. 99, pp. 1-1, 2017.

[99] G. W. Hart, "Nonintrusive appliance load monitoring,"Proc. IEEE, vol. 80, no. 12, pp. 1870-1891, Dec. 1992.

[100] G. W. Hart, "Residential energy monitoring and computerized surveillance via utility power flows," IEEE Technol. Soc. Mag., vol. 8, no. 2, pp. 12-16, Jun. 1989.

[101] J. G. Roos, I. E. Lane, E. C. Botha, and G. P. Hancke,"Using neural networks for non-intrusive monitoring of industrial electrical loads," in Conference Proceedings.10th Anniversary. IMTC/94. Advanced Technologies in IM. 1994 IEEE Instrumentation and Measurement Technolgy Conference (Cat. No.94CH3424-9), 1994, pp.1115-1118 vol.3.

[102] H. T. Yang, H. H. Chang, and C. L. Lin, "Design a Neural Network for Features Selection in Non-intrusive Monitoring of Industrial Electrical Loads," in 2007 11th International Conference on Computer Supported Cooperative Work in Design, 2007, pp. 1022-1027.

[103] Y. H. Lin and M. S. Tsai, "A novel feature extractionmethod for the development of nonintrusive load monitoring system based on BP-ANN," in 2010International Symposium on Computer, Communication, Control and Automation (3CA), 2010, vol. 2, pp. 215-218.

[104] J. Z. Kolter and M. J. Johnson, "REDD: A public data setfor energy disaggregation research," in Workshop onData Mining Applications in Sustainability (SIGKDD),San Diego, CA, 2011, vol. 25, pp. 59-62.

[105] N. Batra et al., "NILMTK: an open source toolkit fornon-intrusive load monitoring," 2014, pp. 265-276.

[106] O. Parson, "Unsupervised training methods for nonintrusiveappliance load monitoring from smart meterdata," University of Southampton, 2014.

[107] M. Chen, S. C. Liew, Z. Shao, and C. Kai, "MarkovApproximation for Combinatorial NetworkOptimization,” IEEETrans. Inf. Theory, vol. 59, no. 10,pp. 6301-6327, Oct. 2013. 
[108] I. Rouvellou and G. W. Hart, "Topology identificationfor traffic and configuration management in dynamicnetworks," in Eleventh Annual Joint Conference of theIEEE Computer and Communications Societies, IEEEINFOCOM '92, 1992, pp.21972204 vol.3.

[109] Y. H. Lin and M. S. Tsai, "Non-Intrusive LoadMonitoring by Novel Neuro-Fuzzy ClassificationConsidering Uncertainties," IEEE Trans. Smart Grid,vol. 5, no. 5, pp. 2376-2384, Sep. 2014.

[110] R. Bonfigli, E. Principi, S. Squartini, M. Fagiani, M.Severini, and F. Piazza, "Useraided footprint extractionfor appliance modelling in Non-Intrusive LoadMonitoring," in 2016 IEEE Symposium Series onComputational Intelligence (SSCI),2016, pp. 1-8.

[111] W. Kong, Z. Y. Dong, D. J. Hill, F. Luo, and Y. Xu, "Improving Nonintrusive Load Monitoring Efficiencyvia a Hybrid Programing Method," IEEE Trans. Ind.Inform., vol. 12, no. 6, pp. 2148-2157, Dec. 2016.

[112] S. Makonin, F. Popowich, I. V. Baji , B. Gill, and L.Bartram, "Exploiting HMM Sparsity to Perform OnlineReal-Time Nonintrusive Load Monitoring," IEEE Trans.Smart Grid, vol. 7, no. 6, pp. 2575-2585, Nov. 2016.

[113] D. Egarter, V. P. Bhuvana, and W. Elmenreich, "PALDi:Online Load Disaggregation via Particle Filtering," IEEETrans.Instrum. Meas., vol. 64, no. 2, pp. 467-477, Feb.2015.

[114] L. Mauch and B. Yang, "A novel DNN-HMM-basedapproach for extracting single loads from aggregate power signals," in 2016 IEEE International Conferenceon Acoustics, Speech and Signal Processing (ICASSP),2016, pp. 2384-2388.

[115] J. Stack, R. G. Harley, P. Springer, and J. A. Mahaffey,"Estimation of Wooden Cross-Arm Integrity UsingArtificial Neural Networks and Laser Vibrometry," IEEE Power Eng. Rev., vol. 22, no. 12, pp. 66-66, Dec. 2002.

[116] Yunus, K.; Thiringer, T.; Chen, P. ARIMA-Based Frequency-Decomposed Modeling of Wind Speed Time Series. IEEE Trans. PowerSyst. 2016, 2546, 25562016.

[117] X. Chen, W. Shen, M. Dai, Z. Cao, J. Jin, and A. Kapoor, "Robust Adaptive SlidingMode Observer Using RBF Neural Network for Lithium-Ion Battery State of Charge Estimation in Electric Vehicles," in IEEE Transactions on Vehicular Technology, vol. 65, no. 4, pp. 1936-1947, April 2016.

[118] Z. Yun, Z. Quan, S. Caixin, L. Shaolan, L. Yuming and S. Yang, "RBF Neural Network and ANFIS-Based Short-Term Load Forecasting Approach in Real-Time Price Environment," in IEEE Transactions on Power Systems, vol. 23, no. 3, pp. 853858, Aug. 2008.

[119] Kong, W.; Dong, Z.Y.; Hill, D.J.; Luo, F.; Xu, Y. Short-Term Residential Load Forecasting Based on Resident Behavior Learning. IEEE Trans. Power Syst. 2018, 1087, 1088-2018. 
[120] A. Khotanzad, Enwang Zhou, and H. Elragal, "A neuro-fuzzy approach to short-term load forecasting in a price-sensitive environment," in IEEE Transactions on Power Systems, vol. 17, no. 4, pp. 1273-1282, Nov. 2002.

[121] S. B. Rout, S. Mishra and S. Mishra, "A review on application of artificial neural network(ANN) on protein secondary structure prediction," 2017 Second International Conference on Electrical, Computer and Communication Technologies (ICECCT), Coimbatore, 2017, pp. 1-6.

[122] Thanchanok Teeraratkul; Daniel O'Neill; Sanjay Lall "Shape Based Approach to Household Electric Load Curve Clustering and Prediction,"in IEEE Transactions on Smart Grid.

[123] A. Ahmad, N. Javaid, M. Guizani, N. Alrajeh and Z. A. Khan, "An Accurate and Fast Converging Short-Term Load Forecasting Model for Industrial Applications in a Smart Grid," in IEEE Transactions on Industrial Informatics, vol. 13, no. 5, pp. 25872596, Oct. 2017.

[124] N. Liu, J. Wang, X. Yu, and L. Ma, "Hybrid Energy Sharing for Smart Building Cluster With CHP System and PV Prosumers: A Coalitional Game Approach," in IEEE Access, vol. 6, pp. 34098-34108, 2018.

[125] K. Mansiri, S. Sukchai and C. Sirisamphanwong, "Fuzzy Control Algorithm for Battery Storage and Demand Side Power Management for Economic Operation of the Smart Grid System at Naresuan University, Thailand," in IEEE Access, vol. 6, pp. 32440-32449, 2018.

[126] K. L. López, C. Gagné and M. A. Gardner, "Demand-Side Management using Deep Learning for Smart Charging of Electric Vehicles," in IEEE Transactions on Smart Grid.

[127] J. Flottemesch and M. Rother, "Optimized energy exchange in primary distribution networks with DC links," 2004 IEEE International Conference on Electric Utility Deregulation, Restructuring and Power Technologies. Proceedings, 2004, pp. 108116 Vol.1.

[128] Marino, D.L.; Amarasinghe, K.; Manic, M. Building energy load forecasting using Deep Neural Networks. In Proceedings of the IECON 2016-42nd Annual Conference of the IEEE Industrial Electronics Society, Florence, Italy, 24-27 October 2016; pp. 7046-7051.

[129] Shi, H.; Xu, M.; Li, R. Deep Learning for Household Load Forecasting - A Novel Pooling Deep RNN. IEEE Trans. Smart Grid 2017.

[130] H. Shi, M. Xu, and R. Li, "Deep Learning for Household Load Forecasting; A Novel Pooling Deep RNN," IEEE Trans. Smart Grid, vol. PP, no. 99, pp. 1-1, 2017.

[131] A. F. Ebrahim and O. Mohammed, "Household Load Forecasting Based on a PreProcessing Non-Intrusive Load Monitoring Techniques," 2018 IEEE Green Technologies Conference (GreenTech), Austin, TX, 2018, pp. 107-114.

[132] George. W. Hart, "Nonintrusive appliance load monitoring," Proc. IEEE, vol. 80, no. 12, pp. 1870-1891, Dec. 1992. George. W. Hart, "Residential energy monitoring and 
computerized surveillance via utility power flows," IEEE Technol. Soc. Mag., vol. 8, no.2, pp. 12-16, Jun. 1989.

[133] George. W. Hart, "Residential energy monitoring and computerized surveillance via utility power flows," IEEE Technol. Soc. Mag., vol. 8, no.2, pp. 12-16, Jun. 1989.

[134] Sung. W. Park, Lee. B. Baker and Paul. D. Franzon, "ApplianceIdentification Algorithm for a Non-Intrusive Home Energy Monitor usingCogent Confabulation," in IEEE Transactions on Smart Grid.

[135] T. Hassan, F. Javed, and N. Arshad, "An Empirical Investigation of V-ITrajectory Based Load Signatures for Non-Intrusive Load Monitoring,"in IEEE Transactions on Smart Grid, vol. 5, no. 2, pp. 870-878, March2014.

[136] M. S. Tsai and Y. H. Lin, "Development of a non-intrusive monitoringtechnique for appliance' identification in electricity energymanagement," 2011 International Conference on Advanced Power SystemAutomation and Protection, Beijing, 2011, pp. 108-113.

[137] N. Batra, Kelly. J, Parson. O, Dutta. H, Knottenbelt. W, Rogers. A Singh.A Srivastava. M, "NILMTK: an open source toolkit for non-intrusive loadmonitoring," 2014, pp. 265-276.

[138] J. Liao, G. Elafoudi, L. Stankovic, and V. Stankovic, "Non-intrusiveappliance load monitoring using low-resolution smart meter data," 2014IEEE International Conference on Smart Grid Communications(SmartGridComm), Venice, 2014, pp. 535-540.

[139] M. Chen, S. C. Liew, Z. Shao and C. Kai, "Markov Approximation forCombinatorial Network Optimization," 2010 Proceedings IEEEINFOCOM, San Diego, CA, 2010, pp. 1-9.

[140] H. Flores and S. N. Srirama, "Dynamic configuration of mobile cloudmiddleware based on traffic load," 2012 IEEE 9th InternationalConference on Mobile Ad-Hoc and Sensor Systems (MASS 2012), LasVegas, NV, 2012, pp. 475-476.

[141] Yu-Hsiu Lin and Men-Shen Tsai, "Application of neuro-fuzzy patternrecognition for Non-intrusive Appliance Load Monitoring in electricityenergy conservation," 2012 IEEE International Conference on FuzzySystems, Brisbane, QLD, 2012, pp. 1-7.

[142] Roberto Bonfigli; Emanuele Principi; Stefano Squartini; MarcoFagiani; Marco Severini; Francesco. P, "User-aided footprint extractionfor appliance modeling in Non-Intrusive Load Monitoring," in 2016 IEEESymposium Series on Computational Intelligence (SSCI), 2016, pp. 1-8.

[143] H. H. Chang, L. S. Lin, N. Chen and W. J. Lee, "Particle-Swarm-Optimization-Based Nonintrusive Demand Monitoring and LoadIdentification in Smart Meters," in IEEE Transactions on IndustryApplications, vol. 49, no. 5, pp. 2229-2236, Sept.-Oct. 2013.

[144] Weicong. Kong, Zhao. Y. Dong, David. J. Hill, Fengji. Luo and Yan. Xu,"Improving Nonintrusive Load Monitoring Efficiency via a HybridProgramming Method," in IEEE Transactions on Industrial Informatics,vol. 12, no. 6, pp. 2148-2157, Dec. 2016. 
[145] Kelly, J.; Knottenbelt, W. "The UK-DALE dataset, domestic appliancelevelelectricity demand, and whole-house demand from five UK homes,"Sci. Data 2015, 2, 150007.

[146] N. Buneeva and A. Reinhardt, "AMBAL: Realistic load signaturegeneration for load disaggregation performance evaluation," 2017 IEEEInternational Conference on Smart Grid Communications(SmartGridComm), Dresden, 2017, pp. 443-448.

[147] Ahmed F. Ebrahim, Osama. Mohammed, "Pre-Processing of EnergyDemand Disaggregation Based Data Mining Techniques for Household Load Demand Forecasting." Inventions 2018, 3, 45.

[148] Yunus, K.; Thiringer, T.; Chen, P. ARIMA-Based Frequency-Decomposed Modeling of Wind Speed Time Series. IEEE Trans. Power Syst. 2016, 31, 25462556. [CrossRef]

[149] Jiang, H.; Zhang, Y.; Muljadi, E.; Zhang, J.; Gao,W. A short-term and highresolution distribution system load forecasting approach using support vector regression with hybrid parameters optimization. IEEE Trans. Smart Grid 2016. [CrossRef]

[150] Abu-Elanien, A.E.B.; Salama, M.M.A. A Wavelet-ANN Technique for Locating Switched Capacitors in Distribution Systems. IEEE Trans. Power Deliv. 2009, 24, 400-409. [CrossRef]

[151] Korolko, N.; Sahinoglu, Z.; Nikovski, D. Modeling and Forecasting Self-Similar Power Load Due to EV Fast Chargers. IEEE Trans. Smart Grid 2016, 7, 1620-1629. [CrossRef]

[152] Zhang, P.; Zhou, X.; Pelliccione, P.; Leung, H. RBF-MLMR: A Multi-Label Metamorphic Relation Prediction Approach Using RBF Neural Network. IEEE Access 2017, 5, 21791-21805. [CrossRef]

[153] Zhang, Y.; Zhou, Q.; Sun, C.; Lei, S.; Liu, Y.; Song, Y. RBF Neural Network and ANFIS-Based Short-Term Load Forecasting Approach in Real-Time Price Environment. IEEE Trans. Power Syst. 2008, 23, 853-858.[CrossRef]

[154] Kong,W.; Dong, Z.Y.; Hill, D.J.; Luo, F.; Xu, Y. Short-Term Residential Load Forecasting Based on Resident Behaviour Learning. IEEE Trans. Power Syst. 2018, 33, 1087-1088. [CrossRef]

[155] Hippert, H.S.; Pedreira, C.E.; Souza, R.C. Neural networks for short-term load forecasting: A review and evaluation. IEEE Trans. Power Syst. 2001, 16, 44-55. [CrossRef]

[156] Ahmad, A.S.; Hassan, M.Y.; Abdullah, M.P.; Rahman, H.A.; Hussin, F.; Abdullah, H.; Saidur, R. A review on applications of ANN and SVM for building electrical energy consumption forecasting. Renew. Sustain. Energy Rev. 2014, 33, 102-109. [CrossRef]

[157] Teeraratkul, T.; O’Neill, D.; Lall, S. Shape-Based Approach to Household Electric Load Curve Clustering andPrediction. IEEE Trans. Smart Grid 2017. [CrossRef] 
[158] Ahmad, A.; Javaid, N.; Guizani, M.; Alrajeh, N.; Khan, Z.A. An Accurate and Fast Converging Short-TermLoad Forecasting Model for Industrial Applications in a Smart Grid. IEEE Trans. Ind. Inform. 2017,13, 2587-2596. [CrossRef]

[159] Ouammi, A. Optimal Power Scheduling for a Cooperative Network of Smart Residential Buildings. IEEE Trans. Sustain. Energy 2016, 7, 1317-1326. [CrossRef]

[160] Ahmed, N.; Levorato, M.; Li, G.-P. Residential Consumer-Centric Demand Side Management. IEEE Trans. Smart Grid 2017. [CrossRef]

[161] Ryu, M.S.; Noh, M.J.; Kim, H. Deep Neural Network Based Demand Side ShortTerm Load Forecasting. In Proceedings of the 2016 IEEE International Conference on Smart Grid Communications (SmartGridComm), Sydney, NSW, Australia, 6-9 November 2016; p. 6.

[162] Tian, P.; Xiao, X.; Wang, K.; Ding, R. A Hierarchical Energy Management System Based on HierarchicalOptimization for Microgrid Community Economic Operation. IEEE Trans. Smart Grid 2016, 7, 2230-2241.[CrossRef]

[163] Marino, D.L.; Amarasinghe, K.; Manic, M. Building energy load forecasting using Deep Neural Networks. In Proceedings of the IECON 2016-42nd Annual Conference of the IEEE Industrial Electronics Society, Florence, Italy, 24-27 October 2016; pp. 7046-7051.

[164] Shi, H.; Xu, M.; Li, R. Deep Learning for Household Load Forecasting-A Novel Pooling Deep RNN. IEEE Trans. Smart Grid 2017. [CrossRef]

[165] Youssef, T.A.; Hariri, M.E.; Elsayed, A.T.; Mohammed, O.A. A DDS-Based Energy Management Framework for Small Microgrid Operation and Control. IEEE Trans. Ind. Inform. 2018, 14, 958-968. [CrossRef]

[166] Elsied, M.; Oukaour, A.; Youssef, T.; Gualous, H.; Mohammed, O. An advanced real-time energy management system for microgrids. Energy 2016, 114, 742-752. [CrossRef]

[167] Hart, G.W. Nonintrusive appliance load monitoring. Proc. IEEE 1992, 80, 18701891. [CrossRef]

[168] Hart, G.W. Residential energy monitoring and computerized surveillance via utility power flows. IEEE Technol. Soc. Mag. 1989, 8, 12-16. [CrossRef]

[169] Roos, J.G.; Lane, I.E.; Botha, E.C.; Hancke, G.P. Using neural networks for nonintrusive monitoring of industrial electrical loads. In Proceedings of the 1994 IEEE Instrumentation and Measurement Technolgy Conference (Cat. No.94CH3424-9), 10th Anniversary IMTC/94 Advanced Technologies in I \& M, Hamamatsu, Japan, 10-12 May 1994; Volume 3, pp. 1115-1118.

[170] Yang, H.T.; Chang, H.H.; Lin, C.L. Design a Neural Network for Features Selection in Non-intrusiveMonitoring of Industrial Electrical Loads. In Proceedings of the 2007 11th International Conference on Computer Supported Cooperative Work in Design, Melbourne, VIC, Australia, 26-28 April 2007; pp. 1022-1027.

[171] Lin, Y.H.; Tsai, M.S. A novel feature extraction method for the development of nonintrusive load monitoring system based on BP-ANN. In Proceedings of the 2010 
International Symposium on Computer, Communication, Control and Automation (3CA), Tainan, Taiwan, 5-7 May 2010; Volume 2, pp. 215-218.

[172] Kelly, J.; Knottenbelt, W. The UK-DALE dataset, domestic appliance-level electricity demand and whole-house demand from five UK homes. Sci. Data 2015, 2, 150007. [CrossRef] [PubMed]

[173] Batra, N.; Kelly, J.; Parson, O.; Dutta, H.; Knottenbelt, W.; Rogers, A.; Singh, A.; Srivastava, M. NILMTK: An open source toolkit for non-intrusive load monitoring. In Proceedings of the Fifth International Conference on Future Energy Systems (ACM e-Energy), Cambridge, UK, 11-13 June 2014; pp. 265-276.

[174] Parson, O. Unsupervised Training Methods for Non-Intrusive Appliance Load Monitoring from Smart Meter Data. Doctoral Thesis, University of Southampton, Southampton, UK, 2014.

[175] Chen, M.; Liew, S.C.; Shao, Z.; Kai, C. Markov Approximation for Combinatorial Network Optimization. IEEE Trans. Inf. Theory 2013, 59, 6301-6327. [CrossRef]

[176] Rouvellou, I.; Hart, G.W. Topology identification for traffic and configuration management in dynamicnetworks. In Proceedings of the Eleventh Annual Joint Conference of the IEEE Computer and Communications Societies, IEEE INFOCOM '92, Florence Italy, 4-8 May 1992; Volume 3, pp. 2197-2204.

[177] Lin, Y.H.; Tsai, M.S. Non-Intrusive Load Monitoring by Novel Neuro-Fuzzy Classification Considering Uncertainties. IEEE Trans. Smart Grid 2014, 5, 23762384. [CrossRef]

[178] Bonfigli, R.; Principi, E.; Squartini, S.; Fagiani, M.; Severini, M.; Piazza, F. Useraided footprint extraction for appliance modelling in Non-Intrusive Load Monitoring. In Proceedings of the 2016 IEEE Symposium Series on Computational Intelligence (SSCI), Athens, Greece, 6-9 December 2016; pp. 1-8.

[179] Kong,W.; Dong, Z.Y.; Hill, D.J.; Luo, F.; Xu, Y. Improving Nonintrusive Load Monitoring Efficiency via a Hybrid Programing Method. IEEE Trans. Ind. Inform. 2016, 12, 2148-2157. [CrossRef]

[180] Makonin, S.; Popowich, F.; Baji'c, I.V.; Gill, B.; Bartram, L. Exploiting HMM Sparsity to Perform Online Real-Time Nonintrusive Load Monitoring. IEEE Trans. Smart Grid 2016, 7, 2575-2585. [CrossRef]

[181] Egarter, D.; Bhuvana, V.P.; Elmenreich, W. PALDi: Online Load Disaggregation via Particle Filtering. IEEE Trans. Instrum. Meas. 2015, 64, 467-477. [CrossRef]

[182] Mauch, L.; Yang, B. A novel DNN-HMM-based approach for extracting single loads from aggregate power signals. In Proceedings of the 2016 IEEE International Conference on Acoustics, Speech and Signal Processing (ICASSP), Shanghai, China, 20-25 March 2016; pp. 2384-2388.

[183] Pereira, L.; Nunes, N.J. A Comparison of Performance Metrics for Event Classification in Non-Intrusive Load Monitoring. In Proceedings of the 2017 IE International Conference on Smart Grid Communications, Dresden, Germany, 2327 October 2017. 
[184] Zhang, L.; Liu, Y.; Chen, G.; He, X.; Guo, X. Assessment Metrics for Unsupervised Non-intrusive Load Disaggregation Learning Algorithms. In Practical Applications of Intelligent Systems; Wen, Z., Li, T., Eds.; Springer: Berlin/Heidelberg, Germany, 2014; Volume 279, pp. 197-206.

[185] Ceperic, E.; Ceperic, V.; Baric, A. A Strategy for Short-Term Load Forecasting by Support Vector Regression Machines. IEEE Trans. Power Syst. 2013, 28, 43564364. [CrossRef]

[186] Li, S.;Wang, P.; Goel, L. A NovelWavelet-Based Ensemble Method for Short-Term Load Forecasting with Hybrid Neural Networks and Feature Selection. IEEE Trans. Power Syst. 2016, 31, 1788-1798. [CrossRef]

[187] Cvetkovic, I.; Boroyevich, D.;Mattavelli, P.; Lee, F.C.; Lucia, O.; Sarnago, H. Design of Home Appliances for a DC-Based Nanogrid System: An Induction Range Study Case. IEEE J. Emerg. Sel. Top. Electron. 2013, 1, 315-326.

[188] Parchure, A.; Tyler, S.J.; Peskin, M.A.; Rahimi, K.; Broadwater, R.P.; Dilek, M. Investigating PV Generation Induced Voltage Volatility for Customers Sharing a Distribution Service Transformer. IEEE Trans. Ind. Appl. 2017, 53, 71-79. [CrossRef]

[189] Chamana, M.; Chowdhury, B.H.; Jahanbakhsh, F. Distributed Control of Voltage Regulating Devices in the Presence of High PV Penetration to Mitigate Ramp-Rate Issues. IEEE Trans. Smart Grid 2018, 9, 1086-1095.[CrossRef]

[190] Wang, Y.; Zhang, N.; Li, H.; Yang, J.; Kang, C. Linear three-phase power flow for unbalanced active distribution networks with PV nodes. CSEE J. Power Energy Syst. 2017, 3, 321-324. [CrossRef]

[191] Alam,M.J.E.;Muttaqi,K.M.; Sutanto,D.Community $\quad$ Energy Storage forNeutralVoltage RiseMitigation in Four-Wire Multigrounded LV Feeders with Unbalanced Solar PV Allocation. IEEE Trans. Smart Grid 2015, 6, 28452855.[CrossRef]

[192] Nallusamy, S.; Parvathyshankar, D.; Velayutham, D.; Govindarajan, U. Power quality improvement in a low-voltage DC ceiling grid powered system. IET Power Electron. 2015, 8, 1902-1911. [CrossRef]

[193] Wunder, B.; Ott, L.; Szpek, M.; Boeke, U.; Weiß, R. Energy e_cient DC-grids for commercial buildings. In Proceedings of the 2014 IEEE 36th International Telecommunications Energy Conference (INTELEC), Sao Paulo, Brazil, 17-20 August 2014; pp. 1-8.

[194] Peña-Alzola, R.; Bianchi, M.A.; Ordonez, M. Control Design of a PFC with Harmonic Mitigation Function for Small Hybrid AC/DC Buildings. IEEE Trans. Power Electron. 2016, 31, 6607-6620. [CrossRef]

[195] Nilsson, D.; Sannino, A. E_ciency analysis of low-and medium-voltage DC distribution systems. In Proceedings of the Power Engineering Society General Meeting, Denver, CO, USA, 6-10 June 2004; pp. 2315-2321.

[196] Arrillaga, J.;Watson, N.R. Power System Harmonics; John Wiley and Sons: Hoboken, NJ, USA, 2004. 
[197] Ahmed, F.; Ebrahim, T.; Youssef, A.; Mohammed, O.A. Power Quality Improvements for Integration of Hybrid AC/DC Nanogrids to Power Systems. In Proceedings of the 2017 Ninth Annual IEEE Green Technologies Conference (GreenTech), Denver, CO, USA, 29-31 March 2017; pp. 171-176.

[198] Kraja ci'c, G.; Dui'c, N.; Vujanovi'c, M.; K1lk1, s, ,S.; Rosen, M.A.; Al-Nimr, M.A. Sustainable development ofenergy, water, and environmental systems for future energy technologies and concepts. Energy Convers. Manag. 2016, 125, 1-14. [CrossRef]

[199] Chowdhury, B.H. Power Quality. IEEE Potentials 2001, 20, 5-11. [CrossRef]

[200] Munir, S.; Li, Y.W. Residential Distribution System Harmonic Compensation Using PV Interfacing Inverter. IEEE Trans. Smart Grid 2013, 4, 816-827. [CrossRef]

[201] Wada, K.; Fujita, H.; Akagi, H. Considerations of an active shunt filter based on voltage detection for installation on a long distribution feeder. IEEE Trans. Ind. Appl. 2002, 38, 1123-1130. [CrossRef]

[202] Lee, T.-L.; Cheng, P.-T.; Akagi, H.; Fujita, H. A Dynamic Tuning Method for Distributed Active Filter Systems. IEEE Trans. Ind. Appl. 2008, 44, 612-623. [CrossRef]

[203] Cheng, P.-T.; Lee, T.-L. Distributed Active Filter Systems (DAFSs): A New Approach to Power System Harmonics. IEEE Trans. Ind. Appl. 2006, 42, 13011309. [CrossRef]

[204] Ward, D.J. The impact of distribution system design on harmonic limits. In Proceedings of the Power Engineering Society 1999 Winter Meeting, New York, NY, USA, 31 January-4 February 1999; Volume 2, pp. 1110-1114.

[205] Haidar,A.M.A.;Muttaqi, K.M.; Sutanto,D. Technical challenges for electric power industries due to grid-integrated electric vehicles in low voltage distributions: A review. Energy Convers. Manag. 2014, 86, 689-700. [CrossRef]

[206] López-Martín, V.M.; Azcondo, F.J.; Pigazo, A. Power Quality Enhancement in Residential Smart Grids Through Power Factor Correction Stages. IEEE Trans. Ind. Electron. 2018, 65, 8553-8564. [CrossRef]

[207] Illindala, M.; Venkataramanan, G. Frequency/Sequence Selective Filters for Power Quality Improvement in a Microgrid. IEEE Trans. Smart Grid 2012, 3, 2039-2047. [CrossRef]

[208] Corasaniti, V.F.; Barbieri, M.B.; Arnera, P.L. Compensación con filtro activo de potencia hibrido en una planta industrial. In Proceedings of the ARGENCON Congreso Bienal de IEEE Argentina, Córdoba, Argentina, 13-15 June 2012.

[209] Bhattacharya, A.; Chakraborty, C.; Bhattacharya, S. Parallel-Connected Shunt Hybrid Active Power Filters Operating at Different Switching Frequencies for Improved Performance. IEEE Trans. Ind. Electron. 2012, 59, 4007-4019. [CrossRef]

[210] Al Sayari, N.; Chilipi, R.; Barara, M. An adaptive control algorithm for gridinterfacing inverters in renewable energy based distributed generation systems. Energy Convers. Manag. 2016, 111, 443-452. [CrossRef] 
[211] Hamid, M.I.; Jusoh, A. Reduction of waveform distortion in grid-injection current from single-phase utility interactive PV-inverter. Energy Convers. Manag. 2014, 85, 212-226. [CrossRef]

[212] Guerrero-Rodríguez, N.F.; Rey-Boué, A.B. Modelling, simulation and experimental verification for renewable agents connected to a distorted utility grid using a RealTime Digital Simulation Platform. Energy Convers. Manag. 2014, 84, 108-121. [CrossRef]

[213] Rahman, M.S.; Oo, A.M.T. Distributed multi-agent based coordinated power management and control strategy for microgrids with distributed energy resources. Energy Convers. Manag. 2017, 139, 20-32.[CrossRef]

[214] Wu, J.-C.; Wu, K.-D.; Jou, H.-L.; Wu, Z.-H.; Chang, S.-K. Novel power electronic interface for grid-connected fuel cell power generation system. Energy Convers. Manag. 2013, 71, 227-234. [CrossRef]

[215] Calleja, H.; Jimenez, H. Performance of a grid-connected PV system used as an active filter. Energy Convers. Manag. 2004, 45, 2417-2428. [CrossRef]

[216] Altin, N.; Ozdemir, S. Three-phase three-level grid interactive inverter with fuzzy logic based maximum power point tracking controller. Energy Convers. Manag. 2013, 69, 17-26. [CrossRef]

[217] Salehi, V.; Mohamed, A.; Mazloomzadeh, A.; Mohammed, O.A. Laboratory-Based Smart Power System, Part II: Control, Monitoring, and Protection. IEEE Trans. Smart Grid 2012, 3, 1405-1417. [CrossRef]

[218] Ebrahim Ahmed, F.; Ahmed, S.; Elmasry, S.E.; Mohammed, O.A. Implementation of a PV emulator using programmable DC power supply. SoutheastCon 2015, 2015, $1-7$.

[219] Elsayed, A.; Ebrahim Ahmed, F.; Mohammed, H.; Mohammed, O.A. Design and implementation of AC/DC active power load emulator. SoutheastCon 2015, 2015, $1-5$.

[220] Salehi, V.; Mohamed, A.; Mazloomzadeh, A.; Mohammed, O.A. Laboratory-Based Smart Power System, Part I: Design and System Development. IEEE Trans. Smart Grid 2012, 3, 1394-1404. [CrossRef]

[221] Margoum, E.H.; Krami, N.; Harmouch, F.Z.; Al montaser, H.; Seca, L.; Moreira, C. Design and Control ofSingle Phase Photovoltaic Systems for AC MicroGrid. In Proceedings of the 2016 International Renewableand Sustainable Energy Conference (IRSEC), Marrakech, Morocco, 14-17 November 2016; pp. 1188-1193.[CrossRef]

[222] Margoum, E.H.; Krami, N.; Seca, L.; Moreira, C.; Mharzi, H. Design and Control of Parallel Three PhaseVoltage Source Inverters in Low Voltage AC Microgrid. Adv. Electr. Electron. Eng. 2017, 15, 120-129.[CrossRef]

[223] Jebaraj, L.; Venkatesan, C.; Soubache, I.; Rajan, C.C.A. Application of Di_erential Evolution Algorithm inStatic and Dynamic Economic or Emission Dispatch Problem: A Review. Renew. Sustain. Energy Rev. 2017,77, 1206-1220. [CrossRef] 
[224] Dieu, V.N.; Ongsakul, W.; Polprasert, J. The Augmented Lagrange Hopfield Network for Economic Dispatchwith Multiple Fuel Options. Math. Comput. Model. 2013, 57, 30-39. [CrossRef]

[225] Secui, D.C. Large-Scale Multi-Area Economic/Emission Dispatch Based on a New Symbiotic OrganismsSearch Algorithm. Energy Convers. Manag. 2017, 154, $203-$ 223. [CrossRef]

[226] Tade, S.V.; Ghate, V.N.; Mulla, S.Q.; Kalgunde, M.N. Application of Dynamic Programming Algorithm forThermal Unit Commitment withWind Power. In Proceedings of the 2018 IEEE Global Conference onWirelessComputing and Networking, GCWCN, Lonavala, India, 23-24 November 2018; pp. 182-186. [CrossRef]

[227] Mohsen, N.; Braun, M.; Tenbohlen, S. Optimization of Unit Commitment and Economic Dispatch inMicrogrids Based on Genetic Algorithm and Mixed Integer Linear Programming. Appl. Energy 2018, 210,944-963.

[228] Moghaddam, M.M.; Marzband, M.; Azarinejadian, F. Optimal Energy Management for a Home MicrogridBased on Multi-Period Artificial Bee Colony. In Proceedings of the 25th Iranian Conference on ElectricalEngineering (ICEE2017), Tehran, Iran, 2-4 May 2017; pp. 1446-1451.

[229] Shi,W.; Li, N.; Chu, C.-C.; Gadh, R. Real-Time Energy Management in Microgrids. IEEE Trans. Smart Grid2017, 8, 228-238. [CrossRef]

[230] Marzband, M.; Ghazimirsaeid, S.S.; Uppal, H.; Fernando, T. A Real-Time Evaluation of Energy ManagementSystems for SmartHybrid Home Microgrids. Electr. Power Syst. Res. 2017, 143, 624-633. [CrossRef]

[231] Netto, R.S.; Ramalho, G.R.; Bonatto, B.D.; Carpinteiro, O.A.S.; Zambroni De Souza, A.C.; Oliveira, D.Q.;Braga, R.A.S. Real-Time Framework for Energy Management System of a Smart Microgrid Using MultiagentSystems. Energies 2018, 11, 656. [CrossRef]

[232] Aragon, V.S.; Esquivel, S.C.; Coello Coello, C.A. Optimizing Constrained Problems through a T-Cell ArtificialImmune System. J. Comput. Sci. Technol. 2008, 8, 158165.

[233] Alberts, B.; Johnson, A.; Lewis, J.; Ra_, M.; Roberts, K.; Walter, P. Helper T Cells and Lymphocyte Activation.In Molecular Biology of the Cell; Garland Science: New York, NY, USA, 2002.

[234] Aragon, V.S.; Esquivel, S.C.; Coello Coello, C.A. A T-Cell Algorithm for Solving Dynamic OptimizationProblems. Inf. Sci. 2011, 181, 3614-3637. [CrossRef]

[235] Aragón, V.S.; Esquivel, S.C.; Coello Coello, C.A. An Immune Algorithm with Power Redistribution forSolving Economic Dispatch Problems. Inf. Sci. 2015, 295, 609-632. [CrossRef]

[236] Harmouch, F.Z.; Krami, N.; Benhaddou, D.; Hmina, N.; Zayer, E.; Margoum, E.H. Survey of MultiagentsSystems Application in Microgrids. In Proceedings of the 2nd International Conference on Electrical andInformation Technologies ICEIT'2016, Tangier, Morocco, 4-7 May 2016; pp. 270-275. 
[237] Mcarthur, S.D.J.; Davidson, E.M.; Catterson, V.M.; Dimeas, A.L.; Hatziargyriou, N.D.; Ponci, F.; Funabashi, T.Multi-Agent Systemsfor Power Engineering Applications - Part I: Concepts, Approaches, and TechnicalChallenges. IEEE Trans. Power Syst. 2007, 22, 1743-1752. [CrossRef]

[238] Harmouch, F.Z.; Krami, N.; Hmina, N. A Multiagent Based Decentralized Energy Management System forPower Exchange Minimization in Microgrid Cluster. Sustain. Cities Soc. 2018, 40, 416-427. [CrossRef]

[239] Pinto, T.; Ghazvini, M.A.F.; Soares, J.; Faia, R.; Corchado, J.M.; Castro, R.; Vale, Z. Decision Support forNegotiations among Microgrids Using a Multiagent Architecture. Energies 2018, 11, 2526. [CrossRef]

[240] Cintuglu, M.H.; Martin, H.; Mohammed, O.A. Real-Time Implementation of Multiagent-Based GameTheory Reverse Auction Model for Microgrid Market Operation. IEEE Trans. Smart Grid 2015, 6, 1064-1072.[CrossRef]

[241] Mahmoudian Esfahani, M.; Hariri, A.; Mohammed, O.A. A Multiagent-Based Game-Theoretic andOptimization Approach for Market Operation of MultiMicrogrid Systems. IEEE Trans. Ind. Inform.2018, 3203, 1-12. [CrossRef]

[242] Logenthiran, T.; Srinivasan, D.; Khambadkone, A.M. Multi-Agent System for Energy Resource Scheduling ofIntegrated Microgrids in a Distributed System. Electr. Power Syst. Res. 2011, 81, 138-148. [CrossRef]

[243] Pinto, T.; Faia, R.; Navarro-Caceres, M.; Santos, G.; Corchado, J.M.; Vale, Z. MultiAgent-Based CBRRecommender System for Intelligent Energy Management in Buildings. IEEE Syst. J. 2019, 13, 1084-1095.[CrossRef]

[244] Anvari-Moghaddam, A.; Rahimi-Kian, A.; Mirian, M.S.; Guerrero, J.M. A MultiAgent Based EnergyManagement Solution for Integrated Buildings and Microgrid System. Appl. Energy 2017, 203, 41-56.[CrossRef]

[245] Bui, V.; Hussain, A.; Kim, H. Optimal Operation of Microgrids Considering AutoConfiguration FunctionUsing Multiagent System. Energies 2017, 10, 1484. [CrossRef]

[246] Choi, I.S.; Hussain, A.; Bui, V.H.; Kim, H.M. A Multi-Agent System-Based Approach for Optimal Operationof Building Microgrids with Rooftop Greenhouse. Energies 2018, 11, 1876. [CrossRef]

[247] Gómez-Expósito, A.; Conejo, J.A.; Cañizares, C. Electric Energy Systems Analysis and Operation; CRC Press:Boca Raton, FL, USA, 2009.

[248] Marzband, M.; Ghadimi, M.; Sumper, A.; Domínguez-garcía, J.L. Experimental Validation of a Real-TimeEnergy Management System Using Multi-Period Gravitational Search Algorithm for Microgrids in IslandedMode. Appl. Energy 2014, 128, 164-174.[CrossRef]

[249] Xiaoping, L.; Ming, D.; Jianghong, H.; Pingping, H.; Yali, P. Dynamic Economic Dispatch for MicrogridsIncluding Battery Energy Storage. IEEE Int. Symp. Power Electron. Distrib. Gener. Syst. 2010, 2, 914-917.[CrossRef] 
[250] Marzband, M.; Parhizi, N.; Savaghebi, M.; Guerrero, J.M. Distributed Smart Decision-Making for aMultimicrogrid System Based on a Hierarchical Interactive Architecture. IEEE Trans. Energy Convers.2016, 31, 637-648. [CrossRef]

[251] Ross, D.; Kim, S. Dynamic Economic Dispatch of Generation. IEEE Trans. Power Appar. Syst. 1980, 99,2060-2068. [CrossRef]

[252] Liang, Z.X.; Glover, J.D. A Zoom Feature for a Dynamic Programming Solution to Economic DispatchIncluding Transmission Losses. IEEE Trans. Power Syst. 1992, 7, 544-550. [CrossRef]

[253] Hemamalini, S.; Simon, S.P. Dynamic Economic Dispatch Using Maclaurin Series Based Lagrangian Method.Energy Convers. Manag. 2010, 51, 2212-2219. [CrossRef]

[254] Li, Z.; Wu, W.; Zhang, B.; Sun, H.; Guo, Q. Dynamic Economic Dispatch Using Lagrangian RelaxationWith Multiplier Updates Based on a Quasi-Newton Method. IEEE Trans. Power Syst. 2013, 28, 4516-4527.[CrossRef]

[255] Shilaja, C.; Ravi, K. Optimization of Emission/Economic Dispatch Using Euclidean A_ne Flower PollinationAlgorithm (EFPA) and Binary FPA (BFPA) in Solar Photo Voltaic Generation. Renew. Energy 2017, 107,550-566. [CrossRef]

[256] Chen, C.; Duan, S.; Cai, T.; Liu, B.; Hu, G. Smart Energy Management System for Optimal MicrogridEconomic Operation. IET Renew. Power Gener. 2011, 5, 258. [CrossRef]

[257] Faia, R.; Pinto, T.; Vale, Z.; Corchado, J.M.; Soares, J.; Lezama, F. Genetic Algorithms for Portfolio Optimizationwith Weighted Sum Approach. In Proceedings of the 2018 IEEE Symposium Series on ComputationalIntelligence, Bangalore, India, 18-21 Novermber 2018; pp. 1823-1829. [CrossRef]

[258] Oliveira, D.Q.; Zambroni de Souza, A.C.; Almeida, A.B.; Lima, I. An Artificial Immune Approach for ServiceRestoration in Smart Distribution Systems. In Proceedings of the 2015 IEEE PES Innovative Smart GridTechnologies Latin America (ISGT LATAM), Montevideo, Uruguay, 5-7 October 2015; pp. 1-6.

[259] Bellifemine, F.; Caire, G.; Greenwood, D. Developing Multi-Agent with JADE Systems; Wooldridge, M., Ed.;John Wiley \& Sons: London, UK, 2007. [CrossRef]

[260] Bellifemine, F.; Caire, G.; Trucco, T.; Rimassa, G.; Mungenast, R. JADE Administrator's Guide; TILab: Turin,Italy, 2010.

[261] RTI DDS. What Is DCPS? Available online: https://community.rti.com/static/documentation/connextdds 15.2.0/doc/manuals/connext_dds/html_files/RTI_ConnextDDS_CoreLibraries_User sManual/Content/ UsersManual/What_is_DCPS_.htm (accessed on 15 June 2019).

[262] RTI DDS. Data Topics-What Is the Data Called? Available online: https://community.rti.com/static/documentation/connextdds/5.2.0/doc/manuals/con next_dds/html_files/RTI_ConnextDDS_CoreLibraries_UsersManual/index.htm\#Us ersManual/Data_Topics_What_is_the_Data_Called_.htm\%3FTocPath\%3DPart $\% 25201 \% 253 \mathrm{~A} \% 2520$ Introduction\%7CDa (accessed on 15 June 2019). 
[263] The Pennsylvania State Climatologist. 2018. Available online: http://climate.psu.edu/ (accessed on22 June 2019).

[264] National Solar Radiation Data Base. 1991. Available online: https://rredc.nrel.gov/solar/old_data/nsrdb/19912010/hourly/siteonthefly.cgi?id=722020 (accessed on 22 June 2019).

[265] Salehi, V.; Mohamed, A.; Member, S.; Mazloomzadeh, A.; Mohammed, O.A. Laboratory-Based Smart Power System, Part I: Design and System Development. IEEE Trans. Smart Grid 2012, 3, 1394-1404. [CrossRef]

[266] Youssef, T.A.; Elsayed, A.T.; Mohammed, O.A. Data Distribution Service-Based Interoperability Framework for Smart Grid Testbed Infrastructure. Energies 2016, 9, 150. [CrossRef]

[267] D. E. O 1 i v a r e s e t al., "Trends i n Microgrid Control," in IEEETransactions on Smart Grid, vol. 5, no. 4, pp. 1905-1919, July 2014.

[268] S. A. Arefifar, Y. A. R. I. Mohamed and T. H. M. El-Fouly, "Supply-AdequacyBased Optimal Construction of Microgrids in SmartDistribution Systems," in IEEE Transactions on Smart Grid, vol. 3, no.3, pp. 1491-1502, Sept. 2012.

[269] C. M. Colson, M. H. Nehrir, "Distributed multi-agent microgrids: adecentralized approach to resilient power system self-healing," 4thInternational Symposium on Resilient Control Systems (ISRCS), pp.83-88, 2011.

[270] I. K. Song; W. W. Jung; J. Y. Kim; S. Y. Yun; J. H. Choi; S. J. Ahn,“Operation Schemes of Smart Distribution Networks With DistributedEnergy Resources for Loss Reduction and Service Restoration," IEEETransactions on Smart Grid, vol. 4, no. 1, pp. 367-374, 2013

[271] D. Wu, F. Tang, T. Dragicevic, J. C. Vasquez and J. M. Guerrero, "AControl Architecture to Coordinate Renewable Energy Sources andEnergy Storage Systems in Islanded Microgrids," in IEEETransactions on Smart Grid, vol. 6, no. 3, pp. 11561166, May 2015.

[272] Z. Wang, B. Chen, J. Wang, M. M. Begovic and C. Chen, "CoordinatedEnergy Management of Networked Microgrids in Distribution Systems,"in IEEE Transactions on Smart Grid, vol. 6, no. 1, pp. 45-53, Jan. 2015.

[273] H. Lotfi; A. Khodaei, "AC Versus DC Microgrid Planning," in IEEETransactions o n Smart Grid, vol.PP, no.99, pp.1-1, 2015, doi: 10.1109/TSG.2015.2457910

[274] T. Dragi evi ，X. Lu, J. C. Vasquez and J. M. Guerrero, "DC Microgrids-Part II: A Review of Power Architectures, Applications, and Standardization Issues," in IEEE Transactions on Power Electronics, vol. 31, no. 5, pp. 3528-3549, May 2016.

[275] G. AlLee and W. Tschudi, "Edison Redux: 380 Vdc Brings Reliability and Efficiency to Sustainable Data Centers," in IEEE Power and Energy Magazine, vol. 10, no. 6, pp. 50-59, Nov.-Dec. 2012.

[276] Ahmed T. Elsayed, Ahmed A. Mohamed and Osama A. Mohammed,"DC microgrids and distribution systems: An overview," Electric Power Systems Research Journal, 119, February 2015, 407-417. 
[277] S. Kahrobaee, R.A. Rajabzadeh, L. Soh, and S. Asgarpoor, "A multiagent modeling and investigation of smart homes with power generation, storage, and trading features," IEEE Transactions on Smart Grid, vol. 4, pp. 659 - 668, 2013

[278] S.D.J. McArthur, E.M. Davidson, V.M. Catterson, A.L. Dimeas, N.D. Hatziargyriou, F. Ponci, and T. Funabashi, "Multi-agent systems for power engineering applications-part I: concepts, approaches, and technical challenges," IEEE Transactions on Power Systems, vol. 22, pp. 1743 -1752, 2007.

[279] P. Wei, Y. Yan, Y. Ni, J. Yen, and F.F. Wu, "A decentralized approach for optimal wholesale cross-border trade planning using multi-agent technology," IEEE Transactions on Power Systems, vol. 16, pp. 833 - 838, 2001.

[280] Yu Chen, Shanshan Zhao, Zuoyu Li, Xiaoguang Wei, and Yong Kang, "Modeling and Control of the Isolated DC-DC Modular Multilevel Converter for Electric Ship Medium Voltage Direct Current Power System," IEEE Journal of Emerging and Selected Topics in Power Electronics, vol. 5, March 2017.

[281] Daniele Bosich, Giorgio Sulligoi, Elena Mocanu, and Madeleine Gibescu,"Medium Voltage DC Power Systems on Ships: An Offline Parameter Estimation for Tuning the Controllers' Linearizing Function," IEEE Transactions on Energy Conversion, vol. 32, June 2017.

[282] J. Millan, "Wide band-gap power s emi co n du c tor devices," IET Circuits, Devices \& Systems, vol. 1, pp. 372 - 379, Oct. 2007.

[283] R. Mitova, R. Ghosh, U. Mhaskar, D. Klikic, W. Miao-Xin and A Dentella. , " Investigations of $600-\mathrm{V}$ GaN HEMT and GaN Diode for Power Converter Applications," IEEE Trans. Power Electronics, vol. 29, pp. 2441 - 2452, Oct. 2013.

[284] Xiucheng Huang, Zhengyang Liu, Qiang Li, and F. C. Lee, "Evaluation and Application of $600 \mathrm{~V}$ GaN HEMT in Cascode Structure," IEEE Trans. Power Electronics, vol. 29, pp. 2453 - 2461, Aug. 2013.

[285] Md. Rabiul Islam, Gang Lei, Youguang Guo, and Jianguo Zhu,“Optimal Design of High-Frequency Magnetic Links for Power Converters Used in Grid-Connected Renewable Energy Systems," in IEEE Trans. MAGNETICS, VOL. 50, NO. 11, NOV. 2014

[286] Xu She, Alex Q. Huang, and Rolando Burgos, "Review of Solid-State Transformer Technologies and Their Application in Power Distribution Systems," IEEE Journal of emer. And Sele. Topics in power electr., vol. 1, no. 3, Sep 2013

[287] Haifeng Fan, and Hui Li, "High-Frequency Transformer Isolated Bidirectional DCDC Converter Modules With High Efficiency Over Wide Load Range for 20 kVA Solid-State Transformer," IEEE Trans. On Ind. Electro., vol. 26, Dec. 2011.

[288] Sachin Madhusoodhanan, Awneesh Tripathi, Dhaval Patel, Krishna Mainali, Arun Kadavelugu, Samir Hazra, Subhashish Bhattac, "Solid-State Transformer and MV Grid Tie Applications Enabled by $15 \mathrm{kV}$ SiC IGBTs and $10 \mathrm{kV}$ SiC MOSFETs Based Multilevel Converters," in IEEE Trans. On Ind. Appli., vol. 51, Aug. 2015. 
[289] M. I. Marei, Nour Elsayed, Ahmed A. El-Sattar, "PV interface system with LVRT capability based on a current controlled HFAC link converter," in Sus. Energy. Techn. and Assess. (Elsevier), Mar. 2015.

[290] Md. Rabiul Islam, Gang Lei, Youguang Guo, and Jianguo Zhu, "Optimal Design of High-Frequency Magnetic Links for Power Converters Used in Grid-Connected Renewable Energy Systems," in IEEE Trans. MAGNETICS, VOL. 50, NO. 11, NOV. 2014

[291] Xu She, Alex Q. Huang, and Rolando Burgos, "Review of Solid-StateTransformer Technologies and Their Application in Power Distribution Systems," IEEE Journal of emer. and Sele. topics in power electr.,vol. 1, no. 3, Sep 2013

[292] Haifeng Fan, and Hui Li, "High-Frequency Transformer Isolated Bidirectional DCDC Converter Modules With High Efficiency Over Wide Load Range for 20 kVA Solid-State Transformer," IEEE Trans. On Ind. Electro., vol. 26, Dec. 2011.

[293] Ahmed. F. Ebrahim, Tarek A. Youssef, Osama A. Mohammed,"Power Quality Improvements for Integration of Hybrid AC/DC Nanogrids to Power Systems" IEEE Greentech 2017, Denver, Colorado, held between March 29 - 31, 2017.

[294] Kyu-Min Cho, Young-Do Kim, In-Ho Cho, and Gun-Woo Moon, "Transformer Integrated With Additional Resonant Inductor for Phase-Shift Full-Bridge Converter With Primary Clamping Diodes," IEEE Trans. Power Electronics, vol. 27, May 2012. 


\section{VITA}

Ahmed Faragallah Farid Ebrahim

Born, Cairo, Egypt

1998-2003

B.Sc., Electrical Engineering, Helwan University, Egypt

2006-2010

M.Sc., Electrical Engineering, Helwan University, Egypt

2016-2019

Graduate Teaching Assistant, Florida International University, Miami, Florida, USA

2019-2020

Dissertation Year Fellowship, Florida International University, Miami, Florida, USA

\section{SELECTED PUBLICATIONS AND PRESENTATIONS}

Format: Journal $[\mathrm{J}-n]$, Conference $[\mathrm{C}-n]$, Patent $[\mathrm{P}-n]$

[J-1] Ahmed. F. Ebrahim, Osama A. Mohammed," Pre-Processing of Energy Demand Disaggregation Based Data Mining Techniques for Household Load Demand Forecasting. Inventions 2018, 3, 45.

[J-2] Ahmed. F. Ebrahim, Ahmed A. Saad, Osama A. Mohammed," Smart Integration of a DC Microgrid: Enhancing the Power Quality Management of the Neighborhood Low-Voltage Distribution Network. Inventions 2019, 4(2), 25.

[J-3] Tawfiq M. Aljohani, Ahmed. F. Ebrahim, Osama A. Mohammed," Single and Multiobjective Optimal Reactive Power Dispatch Based on Hybrid Artificial Physics-Particle Swarm Optimization. Energies 2019, 12(12), 2333.

[J-4] Fatima Zahra Harmouch, Ahmed. F. Ebrahim, Mohammad M. Esfahani, Nissrine Krami, Nabil Hmina, Osama A. Mohammed," An Optimal Energy Management System for Real-Time Operation of Multiagent-Based Microgrids Using a T-Cell Algorithm”. Energies 2019, 12 (5), 3004. 
[C-1] Ahmed. F. Ebrahim and O. A. Mohammed, "Energy Disaggregation Based Deep Learning Techniques: A pre-processing Stage to Enhance the Household Load Forecasting," 2018 IEEE Industry Applications Society Annual Meeting (IAS), Portland, OR, USA, 2018, pp. 1-8.

[C-2] Ahmed. F. Ebrahim, N. Elsayad and O. A. Mohammed, "Medium Voltage DC Testbed: A Hardware-Based Tool to Integrate DC Microgrids/Nanogrids to the Utility Infrastructure," 2018 IEEE Industry Applications Society Annual Meeting (IAS), Portland, OR, USA, 2018, pp. 1-7.

[C-3] Ahmed. F. Ebrahim and O. Mohammed, "Household Load Forecasting Based on a Pre-Processing Non-Intrusive Load Monitoring Techniques," 2018 IEEE Green Technologies Conference (GreenTech), Austin, TX, 2018, pp. 107-114. DOI: $10.1109 /$ GreenTech.2018.00028.

[C-4] Ahmed. F. Ebrahim, A. A. S. Mohamed, A. A. Saad and O. A. Mohammed, "Vector Decoupling Control Design Based on Genetic Algorithm for a Residential Microgrid System for Future City Houses at Islanding Operation," SoutheastCon 2018, St. Petersburg, FL,2018,pp.1-5.doi: 10.1109/SECON.2018.8479013.

[C-5] Ahmed. F. Ebrahim, Tarek A. Youssef, Osama A. Mohammed, " Power Quality Improvements for Integration of Hybrid AC/DC Nanogrids to Power Systems" IEEE Greentech 2017, Denver, Colorado, held between March 29 - 31, 2017.

[C-6] Ahmed. F. Ebrahim, S. M. W. Ahmed, S. E. Elmasry and O. A. Mohammed, "Implementation of a PV emulator using programmable DC power supply," SoutheastCon 2015, Fort Lauderdale, FL, 2015, pp. 1-7. DOI: 10.1109/SECON.2015.7133048.

[C-7] A. Elsayed, Ahmed. F. Ebrahim, H. Mohammed, and O. A. Mohammed, "Design and implementation of $\mathrm{AC} / \mathrm{DC}$ active power load emulator," SoutheastCon 2015, Fort Lauderdale, FL, 2015, pp. 1-5. DOI: 10.1109/SECON.2015.713291.

[C-8] Ahmed. F. Ebrahim, T. Youssef, S. M. W. Ahmed, S. E. Elmasry and O. A. Mohammed, "Fault detection and compensation for a PV system grid-tie inverter," 2014 North American Power Symposium (NAPS), Pullman, WA, 2014, pp. 1-6.doi: 10.1109/NAPS.2014.6965470. 